der deutschen philatelistischen 



 \\ Handbuch \\ der
}

\section{deutschen philatelistischen Literatur}

\author{
8
}

\author{
Im Auftrage des \\ Internationalen Philatelisten $=$ Vereins Dresden
}

unter Mitwirkung von

Oberlandesgerichtsrat Dr. W. Berchelmann, Darmstadt - RedakteurA.E. Glasewald, Gößnitz - Geheimer Regierungsrat Dr. F. Kalckhoff, Berlin - Geheimer Justizrat Emil Pauli, Berlin - Dr. med. Paul Pirl, Charlottenburg - Ernst Plotz, Dresden Dr. jur. O. Rommel, Berlin - K. K. Senatspräsident i. R. Viktor Suppantschitsch, Graz - Franz Wallner, Blasewitz=Dresden

herausgegeben und bearbeitet von

Max Ton.

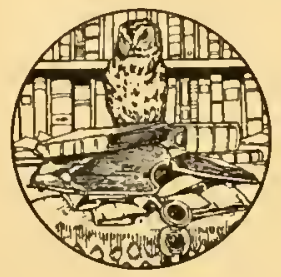

Dresden 1916.

(Als Manuskript gedruckt.)

Franz Wallner, Blasewitz. 



\section{Zur Einführung.}

\section{Inhalt.}

Das Handbuch umfaBt nach Möglichkeit alle von 1863 bis 1916 erschienenen Druckschriften iiber Postwertzeichenliunde und Postgeschichte von fachwissenschaftlichem Interesse.

Deutsche Uebersetzungen fremder Arbeiten sind mit einem Vermerk gekennzeichnet. Hangels wissenschaftlichen Inhalts muften blobe Vereinsberichte, Festschriften u. dgl. vielfach, Vereinssatzungen und Mlitgliederverzeichnisse stets uuberücksichtigt bleiben. Unterhaltungsschriften siudnurausnahms weise wegen ihres philatelistischen Inlalts aufgenommen worden, ebenso Preislisten, Auktionskalaloge und Ausstellungsdrucksacben, soweit sie für philatelistische Forscbungszwecke von Bedeutung sind.

\section{Bibliographie.}

Die Angaben sind tunlichst vollstiindig und genau; insbesondere ist die ursprüngliche Rechtschroibung der alten Titel beibehalten. Nicht oder fätschlich angegebene Verfassernamen sind ergäuzt und berichtigt, und dies kenntlich gemacht durch Anwendung von ekkigen lílammeru ,[ ]“.

\section{Anordnung des Stoffes.}

Der Stoff ist gegliedert in
A. Bücher
B. Zeitschriften.

Zur Erleichterung des Auffindens sei bemerkt, daB:

1. die Bücher unter dem Zunamen ihrer Verfasser, bei einer Mehrzahl von Verfassern unter dem Zunamen des ersten von ihnen, bei fohlendem Zunamen unter dem Sachtitel,

2. Zeitungen unter ihrem Hauptsticliwort,

3. Drucksachen von Vereinen, Ausstellungen usw., soweit sie überhaupt aufgenommen, unter der Firma bzw. dem Sitz des Vereins oder der Ausstellungsleitung

zu suchen sind.

\section{Umfang und Zeit des Erscheinens.}

Der Umfang selbständiger Arbeiten ist tunlichst vermerkt. Peilagen sind besonders erwähnt.

Die Zeit des Frscheinens ist stets der Druckschrift selbst entnommen. Felilt sie, so ist sie durch ${ }^{\theta}$. J.“ gekennzeichnet.

Für die Reihenfolge ist stets die alpuabe. tisclie Anordnung maBgebend gewesen; nu mehrere Auflagen des gleichen Werkes sind nach drr Zeitfolge geordnet.

\section{Verweisungen.}

Sorgfïltige Verweisungen auf die Arbeiten einzelner Länder und Verfasser werden die Brauclibarkeit des Handbuches, insonderheit das schnelle Auffinden der 'Titel erhöhen.

\section{Formate.}

Das Format ist nach der Höhe des Einbanddeckels bestimmt als

$$
\begin{aligned}
& 8^{0}: \text { bis } 25 \mathrm{~cm} \text {; } \\
& 4^{0}: \text { über } 25 \text { bis } 35 \mathrm{~cm} \text {; } \\
& 2^{0}: \text { über } 35 \text { bis } 45 \mathrm{~cm} \text {; } \\
& \text { gr. } 2^{0}: \text { über } 45 \mathrm{~cm} \text {. }
\end{aligned}
$$

Weicht die hergebrachte bibliographische Gröbenbezeichnung davon ab, so ist sie in runden Kilammern zugefügt.

\section{Beispiel bibliographischer Angaben.}

Berlin u. Leipzig: Friedrich Luckhardt (1900) XIV, 372 S. $8^{\circ}$.

bedeutet: das betreffende Werk ist in Verlag von Friedrich Luckhardt 1900 erschienen, seine Seitenzahlen geher von I-XIV und 1-372. Da die Höbe des Einbauddeckels $21^{1 / 2} \mathrm{~cm}$ hetrïgt, ist das Format $8^{\circ}=0 \mathrm{ktav}$. 


\section{Zeichenerklärung.}

\begin{tabular}{|c|c|}
\hline Abb. $=$ Abbildung(en) & fortges. $=$ fortgesetzt \\
\hline Abdr. $=$ Abdruck & Fortsetz. $=$ Fortselzung \\
\hline Abt. $=$ Abteilung & geb. $=$ gebunden \\
\hline Anh. = Anhang & gedr. = gedruckt \\
\hline Anm. $=$ Anmerkungen & Hrsg. = Herausgeber \\
\hline Aufl. = Auflage & hrsg. = herausgegeben \\
\hline Ausg. $=$ Ausgabe & Jg. = Jahrgang \\
\hline Bd. = Band & kart. = kartoniert \\
\hline Bde. = Bände & in Komm. = in Kommissien \\
\hline Bden. $=$ Bïnden & Leinw, = Leinwand \\
\hline bearb $=$ bearbeitet & Lfg. = Lieferung \\
\hline $\begin{array}{l}\text { bed. verm. }=\text { bedeutend ver- } \\
\text { mehrt }\end{array}$ & $\begin{array}{l}\text { Lfgn. = Lieferungen } \\
\text { Lithogr. = Lithographie }\end{array}$ \\
\hline Beil. = Beilage & lithogr. = lithographiert \\
\hline Bildn, = Bildnis & Ms. $=$ Manuskript \\
\hline $\mathrm{Bl} .=\mathrm{Blatt}$ & Nachtr. $=$ Nachtrag \\
\hline bzw. = beriehungsweise & Nr. $=$ Nummer \\
\hline Dr. $=$ Druck & o. J. = ohne Jahr \\
\hline durchges. $=$ durcligeseben & $0.0 .=$ ohne Ort \\
\hline Ebda. = Ebenda & ०. O. и.J. $=$ ohne Ort und Jahr \\
\hline ersch. = erschienen & Orig. = Original \\
\hline erw. = erweitert & l'ortr. = l'erträt \\
\hline$=$ farbig & Pseud. = Pseudonym \\
\hline fortgef. $=$ fortg & Red. $=$ Redakteur, Reda \\
\hline
\end{tabular}

Reg. = Register

s. = siehe

s. u. d. T. $=$ siehe unter dem

Titel

Selbstverl. = Selbstverlag

Sonderabdr. $=$ Sonderabdruck

Sonderdr. = Sonderdruck

Sondert. $=$ Sendertitel

Taf. = Tafel

T. = Teil

Übers, = Übersetzer

übers. $=$ ïbersetzt

ungearb. = umgenrbeitet

Umschl. = Umschlag

Umschlagt. = Umschlagtitel

verb. = verbessert (e)

Verf. $=$ Verfasser

verf. = verfabit

$\mathrm{vgl}$. = vergleiche

verm. = vermehrt(e)

vers. $=$ verseben

Vorw. $=$ Vorwort

zsgest. $=$ zusammengestellt

() = Ergänzungen aus dem Buch oder der Zeitschrift solbst entnommen.

[]$=\quad$ aus fremden Quellen stammend.

\langle\rangle$=$ Kenntlichmachung der auf dem Titelblatt des Buches oder der Zeitschrift bereits eingeklammerten Stellen.

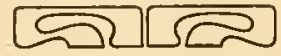




\section{Bücher.}

Abessinien s. Äthiopien.

Alstenueluugsfrage s. Pirl, Paul, Ein Beitrag zur Abstempelungsfrage.

Alhesir-Briefmarken s. Chalmers, Patrick.

Alpßbiicher. AdreBbuch aller Briefmarken. Händler der Welt. s. Senf, Ricliard.

- Adrebbuch der bauptsäehlichsten Briefmarken-Ḧ̈ndler. s. Pegan, Finrico.

- Adrebbuch der bauptsichlichsten Briefmarkenhäudler der gauzen Welt. s. Leh. mann, $\mathrm{H}$.

- Adreßbueh der äberseeisehen u. der orientalischen Briefmarkensanmler. s. Mittelmann, H.

- AdreBbuch europäischer Markenhïndler. s. Mann, Christian, jun.

- Philatelistisebes AdreBbueh. s. Marbes, August.

- Die einzig richtigen Adressen der hauptsächlichsten Briefmarkenhändler. s. Lehmann, H.

- Adressen zur Prüfung und zur Besorgung von Postwertzeichen. Nur für Mitglieder der am Vertraulichen Korrespondenzblatt beteiligten philatelistischen Vereine! (Sonderbeil. z. Vertraul. Korresp.-Blatt philatel. Vereine. 11. Jg. 1901 Nr. 3). Kiel 1901. Druck von Schmidt \& Klaunig. $16 \mathrm{~S} . \mathrm{S}^{0}$.

- 100 Adressen von Briefmarkenbändlern aus allen Weltteilen. s. Kreuchauf, 0 .

- 150 Adressen der momentan wirklich existierenden u. bestrenommierten BriefmarkenHändler u. Sammler. s. Nenze, 0.

- 150 Adressen von anerkannt guten Händlern. s. Halbrock, E.

- 550 Adressen der philatelistisclen Vereine und Zeitungen (Sonderbeil. zum Vertraul. Korresp.-Blatt philat. Vereine. 11. Jg. 190I Nr. 8). Kiel 1901. Druck von Schmidt \& Klaunig. $8^{\circ}$.

- 2150 Adressen der Briefmarken-Hündler und Sammler aller Länder. s. Serbe, C. Hermann.

- Adreß-Verzeichnis der Briefmarken-Händler und Sammler. s. Menze, 0.

- Händlerliste über 100 Adressen enthaltend. s. Braunschweig, Johannes.

- Philatelisten-AdreBbuch. s. Haack.

- Philatelist. Nachschlagebuch. 8. Wenze], E.

- 1nternationales Philatelisten-AdreBbuch mit Angabe der Sammelobjekte, Tausclı-, Taufund Terkaufshedingungeu usw. der Sammler und Händler. Leipzig: Hermann Dege 1902. VII, 188 S. $8^{\circ}$.

Alreßliicher. Internationales PhilatelistenArlebbuch. s. a. GieBmann.

- - s. a. Krehn, Julius \& Löwinger, Mas.

- Internationales Tausch-, AdreB-u. Hilfsbuch für den Tauschverkehr. s. Isakovics, Alois ron.

- Erstes philatelistisches WeltadreBbuch. s. Bruder, Willy. (Pseud. H. J. Dauth.)

- Welt-Briefmarkenhändler-AdreBbuch, s. Owiteb, A.

Afglianistan. Bayer, Eugen: Die Postwertzeichen von Afghanistan. Dejvico.Prag 1916.

- Neyer, Ferdinand: Afghanistan, seine Post und seine Postwertzeichen. Wien 1879.

Afrika. Kobl, Paul: IlIustr. Freimarken-liatalog "Euglische Kolonien". Abt. 2. Kolonien von Afrika.

- s. a. u. d. Einzelstaaten.

Ägypten. Dautlı, H. J.: Abdruck postamtlieher Dokumente. H. 1. Igypten.

- Koch, Gustav. s. Herländer, Lulwig.

- Merländer, Ludwig: Die Administrationsmarken Aegyptens. Alexandrien, Leipzig 1890.

Albanien. Freyse, Gustav: Die Post in Albanien. Hannover 1915/16. [ln Vorbereitung.]

Albrecht and Witt, New Tork: Suchen zu den hierin aufgefülurten Preisen Vereinigte. Staaten-Marken zu kaufen. New Iork. [1892]. $12 \mathrm{~S} .8^{\circ}\left(16^{\circ}\right)$.

Altdentsehland. s. Deutsches Reieh und Einzelstaaten.

Amcrika. s. Konföderierte Staaten von Amerika, Vereinigte Staaten von Nord-Amerika u. Einzelstaaten.

Andresen, Ferdinand, j1.: En gros. Fin detail. Preisliste über norwegische Briefmarken zu beziehen ron F. A. jr. Christiania, Mlai, 1896. 8 S. $8^{0}$.

- Preisliste ïber Skandinarische Briefmarken zu beziehen von F. A. jr. Clristiania, Oktober, 1897. J, $7 \mathrm{~S} .8^{\circ}$.

Anheisser, J. H. s. Teltz, O.: Großes llandbucb der Philatelie. [T.21-27.] 1887 [-97]. 8".

Antoniades, J. T.: 1892-93. Katalog über orientalische Postwertzeichen zusammengestellt nach Meyers und Moschkaus Handbuch für Postmarkensammler und mit billigsten Preisen versehen. Stück, Satz 10, 100, 1000, 5000 u. 10000 Stück. Hrsg. von J. T. A. . Censtantinopel: Selbstverlag 1892. VI, 18.S. $\mathrm{S}^{\circ}$. 
Intoniales, J. 'I': Beschreibender Katalog sïmtlicher orientalischer Postwertzeichen (deutsch u. französisch) mit allen Proisen. Constantinopel: Selbstverlag 1893. 94 S. $8^{\circ}$.

Appel, Sophus: Hin detail Briefmarken-PreisKurant von S.A. Kopenhagen, $1877.1,11 \mathrm{~S} .4^{\circ}$.

Asien. s. u. d. Einzolstaaten.

Ithiopien. Kohl, Paul: Die Postwertzeichen von Äthiopion (Abessinien). Chemnitz 1911.

Aufdrucke. Gottseba]l, Albert: Die Aufdrucke auf den Postmarlien. Berlin, Leipzig [gedruckt 1895.]

Infurneke. Haas, Theodor: Korreferat, betr. Reform des Vordruckalbum und Agitation gegen das Aufdruckunwesen. IVien 1885.

- Vortrag über das Aufdrucksammeln. München 1885.

- Klenze, Heinrich von : Referat, betr. Agitation gegen das Aufdruckunwesen. München 1885.

Auktions-Kataloge. K. k. Versteigcrungsamt Dorotheum Wien. 261. liunstauktion. VII. Briefmarken-Auktion zweicr Europa-Simmlungen aus Privatbesitz, hiervon eine aus den Nachlasse des k. u. k. Hof-Kunsthändlers IIeinrich L. Noumann.

Auktion: 22.-24. März 1916,

Schaustellung: 19.-21. März 1916 in den Kunstauktionssälen des Dorotheums. Wien 1, Dorotheergasse 17: Versteigerungsamt (Markenhaus Rudolf Friedl in Komm.) 1916. 78 S. u. 6 Taf. m. Markenabb. $8^{\circ}$.

- - 264. Kunstauktion. V11I. Briefmarken. auktion einer General-Sammlung aus Wiener Privatbesitz.

Auktion: 29.-31. Mai 1916,

Schaustellung: 25.-27. Mai 1916, in den Kunstauktionssälen des Dorotheums. Wien I, Dorotheergasse 17: Versteigerungsamt (Markenhaus Rudolf Friedl in Komm.) 1916. 68 S. u. 18 Taf. m. Narkenabb. $8^{\circ}$

- lioehler, Heinrich: Katalog der ersten "Briefmarken-Auktion. Stückweise Versteigerung einer Pracht-Sammlung ${ }_{\pi}$ Altdeutschland und Deutscher Kolonialmarken" im Künstlerhaus Berlin W, Bellevuestr. 3. Versteigerungstage: 23.-26. IV. 1913. Berlin: Heinrich Koebler 1913. $8^{\circ}$.

- Katalog der, ,zweiten" Briefmarken-Auktion. Stückweise Versteigerung einor PrachtSammlung "Europa“ im Künstlerhaus Berlin W, Bellevuestr. 3. Versteigerungstage: $3 .-6$. VI. 1913. Berlin: Heinr. Koebler 1913. $47 \mathrm{~S}$. u. 8 Taf. m. Abb. $8^{\circ}$.

- Katalog der dritten" Briefmarken-Auktion. Stückweise Versteigerung einer Prachtsammlung, Altdeutschland ", darunter, Baden. specialsammlung, Oesterreich u. Sizilien", im Künstlerbaus Berlin W, Bellevuestr.3. Versteigerungstage: 18. u. 19. VI. 1913. Berlin: Hoinrich Koehler 1913. 44 S. u. 8 Taf. m. Abb. $8^{\circ}$.
Auktions-Kataloge. Koehler, Heinrich: Katalog der Briefmarken-Auktion Nr. 4 im líriegerheim, Nikolaistr. 10, Hannover. Stückweise Versteigerung aus der l'racht-Sammlung A. Jeanrenaud und aus Privatbesitz stammender Briefm. Versteigerer: Hans v. d. Pordten, Hannover, Schmiedestr. 4. Sachverständiger: Heinr. Koebler, Berlin IV 8, Friedrichstr. 166. Versteigerungstage: $13-15 . V I I I .1913$. Berlin: Heinr. Foobler 1913. 40 S. u. 9 Taf. m. Abb. $8^{\circ}$.

_ Katalog der Briefmarken-Auktion Nr. 5. StückweiseVersteigerung einer Pracht-Sammlung "Altdeutschland "und Rarititen aller Länder im Künstlerhaus Berlin W, BellevuestraBe 3. Versteigerungstage: 4.-6. Xl. 1913. Berlin: Heinrich Koehle! 1913. 71 S. u. $13^{\prime}$ Taf. m. Abb. $8^{\circ}$.

- Katalog der Briefmarken-Auktion Nr. 6. StückweiseVersteigerung einer Pracht-Sammlung "Altdeutschland" und Raritäten aller Liuder im Künstlerhaus Berlin W, Bellevue. straße 3. Versteigerungstage: 16.-18. XII. 1913. Berlin: Heinrich Koehler 1913. $71 \mathrm{~S}$. u. 16 Taf, m. Abb. $8^{\circ}$.

- Katalog der Briefmarken-Auktion Nr. 7. Stückweise Versteigerung einer Pracht-Sammlung , Europ: " und Rariäten aller Länder im Künstlerhaus Berlin W, Bellevuestr. 3. Vorsteigerungstage: 26.-28.1.1914. Berlin: Ileinrich Koehler 1914. 67 S. u. 17 Taf.m.Abb. $8^{\circ}$.

- Katalog der Briefmarken-Auktion Nr. 8. Stückweise Versteigerung einer, Generalsammlung im Ki ïnstlerbaus Berlin W, Bellevuestr. 3. Versteigerungstage: 10.-12. III. 1914. Berlin: Heinrich Koehler 1914. $72 \mathrm{~S}$. u. 12 'Taf. m. Abb. $8^{\circ}$.

- Katalog der Briefmarken-Auktion Nr. 9. Stückweise Versteigerung einer Generalsammlung im Künstlerhaus Berlin W. Belle vuestr. 3. Versteigerungsfage: 4-6. V. 1914. Berlin: Heinrich fioehler 1914. 79 S. u. 16 Taf. m. Abb. $8^{\circ}$.

- Katalog der Briefmarken-Auktion Nr. 10. Stiickweise Versteigerung einer Prachtsamm. lung Altdeutschland (Schwarz-Sammlung)" im Kíñnstlerhaus Berlin W, Bellevuestr. 3. Versteigerungstage: 17.-19. VI. 1914. Berlin: Heinr. Koehler 1914. 79 S. m. 58 Abb. im Text u. 18 'Taf. m. Abb. $8^{\circ}$.

- - Katalog der Briefmarken Auktion Nr. 11. Stückweise Versteigerung einer Prachtsammlung - Altdeutschland" im Kïnstlerhaus Berlin W, Bellevuestr. 3. Versteigerungstage: 10. u. 11.1II, 1915. Berliu: Heinrich Koehler 1915. 48 S. m. 8 Abb. im Text u. 8 Taf. m. $\mathrm{Abb} .8^{\circ}$.

- Kosack, Philipp und M. de Vries: Versteigerung $1-6$. Berlin 1900-1901. 80.

1. am 22. Okt. 1900 .

2. an 22. Nov. 1900 .

3. am 10. Jan. 1901 .

4. am 6. Febr. 1901.

5. am 28. Mä̈rz 1901 .

6. an 11. Sept. 1901. 
Ink1 ions-Kataloge. Haier M., Kurt: Katalog der] Briefmarken - Versteigerung im Architelitenhaus zu Berlin, Wilhelmstrabe 92-93 am 15., 16., 17. Mai (1913). Berlin: SSelbstverlag 1913 ] $100 \mathrm{~S}$. m. Markenabb. Kart. m. Seidensehnur. Quer $8^{\circ}$.

_ _ [Katalog der] Briefmarken-Versteigerung im Arehitektemhaus zu Berlin, Wilhelmstrabe 9:3-93 am 5. bis 10. Januar 1914. [Nebst:] Illustrations-Beilage. Berlin IV 8 , Friedricl! straBe 187/88: M. Kurt Maier. 1914. $64 \mathrm{~s}$. u. 15 Taf. m. Markenabb. Kart. m. Feiden. schmur. Quel $9^{0}$.

_ - LKatalog der] Briefnarken-Versteigerung iu Architektenhaus zu Berlin, WilhelmstraBe 92-93, am 18., 19. u. 20 Mai 19l4. [Nebst:] Illustrationsbejlage Berlin: M. Kurt Maier... 1914. 63 S. u. 24 Taf. m. Markenabb. Kart. m. Seidenschnur. Quer $8^{\circ}$.

- Katalog der Briefmarken-Versteigerung zu Frankfurt a. M., Neue Börse, Börsenstr. 6-10 am 16., 17., 1\%. Oktober (1913) durch Herrn hud. Bangel. Sachverständiger: MI. Kurt Maier, Berlin WV 8, Friedrichstl. 187/88. [Nebst:] Kunstdruckbeilage. Berlin: M1. liurt Haier 1913. 37 S. u. 10 Tafsul vorwiegend farbigen Markenabb. Kart. m. Seidenselinur. Quer $8^{\circ}$.

_ - Katalog der] Briefmarken-Versteigerung im Convent-Garten, zu Hamburg am 25., 26., 27. September (19/3), durch Herrn Karl 'Jhielebar i. Fa.: Jolis, Thiessen. Sachverständiger: 1]. Kurt Maier, Berlin, [Nebst:] lllustrations-Beilage. Berlin: M. Kurt Maier (1913). $4 \mathrm{~S}$ S. u. 15 Taf. m. Markenabb. Kart. m. Seidenschnul. Quer $8^{\circ}$.

- - liatalog der] Briefmarken-Versteigerung im Kriegerheim zu Hannover am 13., 14., 15. August (1913), M. Kurt Maier. Berlin durch Hans v. d. Pordten. Berlin: M. Kurt Maier 1913. 80 S. n. Markenabb. kart. m. Seidenschnur. Quer $8^{\circ}$.

- Vries, 11. de.: Versteigerung 7-13. lierlin $1901-1903.8^{\circ}$.
7. $\operatorname{am}$ 6. Nov. 1901.
S. am 9. Jan, 1902 .
9. am 13. Febr. 1802.
10. am 13. Närz 1902.
11. am 1. Mai 1902 .
12. a $\mathrm{m}$ 11. Sept. 1902 .
13. am 22. Jan. 1903.

- - Versteigerung 1-6 s. Anktions-Kataloge: Kosack, l'hilipp u. M. che Vries.

líolıl, Paul: Illustr. Freimarken-Katalog ${ }_{\text {t }}$ Englische Kolonien", Abt. 3. Kolonien in Australien ...

Iustralien. s. a. u. d. Einzelstaten.

Arenarius, Ferdinand: Briefmarken sind keine Kileinigkeit. Jünchen: Georg D. W. Callwey, 1909. 7. S. u. 1 Taf. mit Abb. $8^{\circ}$.

(61. Flugschrift des Dürerbundes zur ästhetischen Kultur.j
Baden. Entwickelung des Post- und 'Telegraphenwesens im Grobherzogtum Baden 1874-1890. Karlsiuhe 1897.

- Glasewald, A. F.: Die Abstempelungen da. Marken von Baden. GöBnitz 1898.

- - Baden-Stempel-Verzeichnis. GöBnit\% 1894.

- Lindenberg, C.: Die Briefmarken von liaden. Berlin 1894.

- - Die Briefumschläge von Baden. Berlin 1894.

- Postverwallung. Dienst-Instruction fiir die GreBherzogl. Badischen Land postboten. Cartsruhe 1859 . XXII, 30 S. $8^{\circ}\left(16^{\circ}\right)$.

- - Instruetion für die Landpestboten. Carls. rulue $1869.5 .8^{\circ}\left(16^{\circ}\right)$.

- Reitz Edler von Bollheim, G.: Die Postwerthzoichen von Baden. Wien 1880.

- Rommel, 0.: Baden. Leipzig 1893. (Krötzsch, Handb. Bd. 2.)

Baggo, E. v.: Die coursirenden Ganzsachen von Russland nebst Preisverzoichniss. Dorpat: S.lustverI. 1889. $6 \mathrm{~S} .8^{\circ}$.

- -- 2. Aufl. Derpat 1890. 6 S. $8^{\circ}$.

- - auch in: Jeran, 0.: Wegwciser für Verkehrswertzeichen - Sammler. (PbilatelistenFibel).

Balkanstaaten. s. Kiohl, P.: IHustr. Katalog der Freimarken von Europa. Abt. 8.

Balke, Franz: Sellen Schüler Brjefmarlien sammelu? Vortrag, gehalten am XX. Dentschen Philatelisten-Tage GöBnitz-Altenburg, 3. August 1908. Von Lehrer F. B., MiilsenSt.-Jakob. (Sonderdr. aus, PhilatelistenZeitung " Jg. 190S.) Göbnitz ${ }^{*}$ S.-A.: A. E. Glasewald 1908. 8S. $8^{\circ}$.

Bassaher. Friedländer Paul: Bassaher. Berlin 1913.

Baum. s. Friedl und Banm: Intern. Postwertzeichen Ausstel]ung Wien 1890.

Baumbach \& Co. B's. Postwertzeichen-Katalog und Blankoliste zum Universal-BriefmarkenAlbum. Loipzig. Baumbach \& Co. [1S94]. $\because 16 \mathrm{~S} . \mathrm{S}^{0}$.

- Zweite Auf. Leipzig. Baumbach \& Co. [1895]. 217 S. 80.

Bauschke, Gustav: Katalog aller bekannten seit 1840 bis Mitte 1865 emittirten Briefmarken. Unter fremmlicher Nitwirkung der Herren W. Th. Clausius, Dr. Joseph u. W. 'Ih. ]srael, hrsg. von G. B. (in Firma: literarisches Museum). Leipzig. Literarisches Museum 1865. II, $130 \mathrm{~S} .8^{0}$.

- Zweiter Abdr. Leipzig. Literarisches Museum 1S65. $130 \mathrm{~S} .8^{\circ}$.

- Hrster Nachtr. Gescblossen am 1. November 1865 . Leipzig. Literarisches Museum 1865. I, $11 \mathrm{~S} . \mathrm{S}^{0}$.

- Katalog aller bekannten Briefmarken. Unter freundlicher Witwirkung der ersten Samm]erautorititen Deutschlands, hrsg. von C. B. (in Firma: Literariscbes Nuseum). Achte, bis 1867 vervollständigte Anfl. Leipzig. Lite. rarisches Museum 1867. VII, 14S S. $5^{\circ}$. 
Baycr, Eugen: Die Postwertzeichen von Afghanistan. (Sonderdr. aus "Der Philatelist" 1916. Nr. 3 u. 4.) Dejvice-Prag: Selbstverl. des Verf. 19l6. 7. u. 1 Taf.m. Abb. $8^{\circ}$.

Bayer. Bayerischer Briefmarken-SammlerVereiu. Bayern-Neudr. München 1902.

- Brummer, L.: Die Bayerischen Portomarken. Berlin 1898.

- Brunner, J.: Das Postwesen in Bayern etc. München 1900.

- Chelius, A.: Der Specialsammler von Bayeru nach Abstempelungen. Nünchen 1900.

- Friedl, S.: Die Postwerthzeichen von Bajern. Wien I880.

- Hosp, E.: Preis-Liste über bayerische Marken. München [1884]

- Joris, C.: Aufstellung der Couverte, Streifbänder, Postkarten u. Postanweisungen des Königreiches Baiern. München 1883.

- Joris, C. u. Sedlmayr, O.: Geschichte und Katalog der Postwertzeichen des Königreichs Bayern. Minchen 1893.

- Lindenberg, C.: Die Bricfumschläge von Bayern. Berlin 1895.

- Post-Lexikon für das Königreich Bayern. Nach amtlichen Quellen der General-Direktion der K. bayer. Verkehrs-Anstalten. Nïinchen $1868.703 \mathrm{~S} .8^{\circ}$.

- Reinheimer, Ad.: Der bayerische Mïhlradstempel. Leipzig 1832.

- Rommel, O.: Bayern. (Krötzsch, Handb. Bd.3.) Leipzig 1893.

- Schneider, Fr.: Katalog der-Bayerischen Postkarten nebst Preisverzeichnis. Berlin 1915.

- Stallechner, J.: Special-Preisliste Bayrischer Postwerthzeichen. Miesbach [1879].

- Stenger, E.: Bayern. [Berlin] 1912.

- Bayern 9 Kr. grün. Typenunterschiede. [Leipzig] 1914.

- - Bayern - Feldpost 1914. Berlin 1914.

- - Bayern - Portomarke $3 \mathrm{Kr}$. schwarz mit Seidenfaden. Berlin 1910.

- Essais und Proben der Brief-, Porto-, Telegraphen-Stempelmarken und Ganzsachen von Bayern. Berlin 1893.

- Ceschichte und Kratalog der Postanweisungen von Bayern. Leipzig 1914.

- - Die rote Gummierung bei Bayernmarken. Berlin 1914.

- Die Güterexpeditionsstempel von Bayern als Briefstempel. Berlin 1915.

- - Die Mühlradstempel von Bayern. [Leipzig] 1915.

- Die Postliarten ron Bayern. Ein Nachtring zu Fr. Schneider's Katalog nehst Preisvelzeichnis. Berlin 1915.

Stenger, Erich u. Lndwig Sauter: Die Postablagen-, Land postboten- und PosthilfsstellenStempel von Bayern. Berlin 1915.
Bayerisehe Landes-Induslrie-, Gewroce- und funst-Ausstellung iu Niinnber.r. s. Nïmberg.

Bayerischer Briefmnren-Sammler-Verein. s. München,

Bayeriseher Philatelisteu-Vereiu Minehen. 8. Miunclien.

Bayern-Neudruck. Nünchen: Bayerischer Briefmarken-Sammler-Verein 1902. $4 \mathrm{~S} .4^{\circ}$.

Beavregard, George de und If. de Gorsse: Del Briefmarkenkönig. Autorisierte Übprsetzung ans dem Französischen vou 0 . 'T. Alexauder. Berlin: H. J. Meidinger 1899. 295 S. mit 8 Vollbilderu u. mehreren Abb. im 'l'ext. $8^{\circ}$.

Belora. Beitrag zur Geschichte des Postamts B. s. Ruhl, J.

Beck, Car]: Bibliographie der wichtigsten deutschen Sonderschritten über die Postwertzejehen einzelner Länder. Leipzig u. Breslau: [trormania-Ring] 1915. 16 S. $40^{\circ}$.

- Das Reichs-Postmusenm und seine Markenbestände. Vortrag gehalten auf dem XIl. Deutschen Sammlertage zu Potsdam. (Sonderabdr. ans den "Philatelistischen Miscellen".) Berliu: R. Fr. Funcke 1908. $11 \mathrm{~S} .8^{\circ}\left(16^{\circ}\right)$.

- Praktischer Wegweiser durch das Reich der Philatelie. Ein Notbehelf für jüngere Briefmarkensammler nebst einer Geschichte der Deutschen Philatelistentage. Bearb. u. a ach bewährten Quellen zsgest. von C. B.... Berliu: Selbstverl. 189y. 15 S. $8^{\circ}$.

Beck, Carl u. Carl Holzsehuher: Die Bayerischen Mühlrad-Stempel. Wörisbofen: Hans Schneider. 1911. 116 S. m. Abl. $8^{\circ}$.

Beck, 0tto: Weltpost, Reichspost, Mainzer Post. Das Postwesen in Vergangenheit und Gegenwart mit besonderer Berücksichtigung der Mainzer Verhältnisse. Zur Hrinnerung an den Posthans-Neuban in Mainz. 1. März 1909. Mainz: Selbstverl. des Verf. 1909. $36 \mathrm{~S} .8^{\circ}$.

Becker, Hermanu Joseph: Das Postwesen im Saargebiet. Seine Entwickelung nnter dem wechselnden Kinfluss der Kriegs Ereignisse wie des friedlichen Kulturfortschritts innerhalb der letzten drei Jahrhunderte. [Nebst] Anh.: Bedeutung und Tätigkeit der Post im modernen Kriege. Saarbrücken: Chr. Clauss 1915. 56 S. $8^{\circ}$.

Belgien. Kohl, Paul: Illustr. Katalog der Freimarken von Europa. Abt. 2. Belgien...

- P'auli, Emil: Ober den strafrechtlichen Schutz der Postwertzeichen in Belgien ... Berlin 1913.

- Sinuer, K. Cl.: Permanenter BriefmarkenKatalog. Belgien und Kongo-Staat, MährischOstrau [188: ].

Bergelorf. Kiötzsch, Il.: Die Postfreimarken des beiderstädtischen Postamtes Bergedorf. 2. Aufl. ‘hı̈̈tzsch, Handb. Bd.4.) Leipzig 1896.

- Pfaff, C.: Über Bergedorfer Briefmarken auf Grundlacre amtlicher Bestimmungen. Hannover [I $\measuredangle 91]$. 
Bergedorf. Postverwaltung. Abschrift. Bekanntmachung die Einführung von Postmarken zum Frankieren der Briefpostsendungen betreffend. 16 S. $4^{\circ}$. [Autographiert.] Bergedorf [1861].

- Rommel, O.: Bergedorf. 1. Aufl. (Krötzsch, Handb. Bd. 4.) Leipzig 1893

- - Die Postwertzeichen des Bergedorfer Postbezirkes. München 1892.

- - 1. Nachtrag. Müuchen 1892.]

- $-[2 . \quad " \quad$ Dresden 1894.]

- - 3. " " $\quad$ " 1895 .

Berger, II. [d. i. Arthur Wülbern]: Illustrierter Preis-Katalog der Telegrafen- und Stempelmarken der deutschen Einzelstaaten. Bearb. von H. B. Ein Supplement zu jedem Postmarken-Katalog. Hamburg: Arthur Wülbern 1896. 2 L S. u. 7 Taf. m. Ább. $8^{\circ}$.

- Illustrierter Preis-Katalog der Telegratenund Stempelmarken. Bearb. von II. I. (A. Wülbern.) Bd. 2. Österreich-Ungarn und Balkanstaaten. Hamburg: Arthur Wülbern 1898. 41 S. m. Abb. $8^{0}$.

Berger, Lulwig: Die Postwerthzeichen des Herzogthums Braunschweig nebst einem kurzen Abriss der Braunschweigischen Postgeschichte. Unter Benutzung amtlicher Quellen bearb. von L. B. Braunschweig: Selbstverl. 1893. II, 138 S. m. Abb. $8^{\circ}$.

- Portugal. 8. Berlin (Berliner PhilatelistenKlub). in: Festschrift z. Feier des zehnjährig. Bestehens des B. Ph. Kl.

Berger-Levrault, Oscar: Beschreibung der bis jetzt bekannten Briefmarken (melrr als 2200 Sorten), nebst Notizen über die nachgedruckten Marken und einer Anleitung zur Einrichtung von Sammlungen. Strassburg: Wwe. Berger-Levrault \& Sohn 1864. $103 \mathrm{~S}$. $8^{\circ}\left(12^{\circ}\right)$.

Berlin. (Berliner Philatelisten-Klub) B. Ph.-Kl. Bibliothek. Deutsche Zeitschriften. Berlin: [H. Fränkel.] 1894. 16 S. 80 . [Einseitig gedr.]. - Festschrift zur Feier des zehnjährigen Bestehens des Berliner Philatelisten-Kilub. Im Auftrage des Klubs hrsg. von H. Fraenkel, Dr. F. Kalckhoff, C. Lindenberg, P. Ohrt. Berlin: Selbstverl. des B. Ph.-KI. 1898. VI, 271 S. u. 6 Taf. m. Abb. $8^{0}$.

I a halt: Berger, Ludwig: Portagal. Studie über die Ausgshen 1853-1876. - Bochmann, E. r.: Die Schweizer Marken der Emiesion 1854/63. - Brummer, Leon : Die Bayeriechen Porto-Marken. - Csstle, M.P.: Die eltenereo Marken ron Grossbritsunieo. - Diesa, Emilio: Die I Bajocco-Marke des Kirchenatastes Friederich, Rudolf: $\mathrm{Y} 1 /{ }_{4}$. - Gottechslk, Albert: Die Douteche philatelietische Literetur 1894/97. - Hs8s, Theodor: Die Pootwertzeichea des Susheli-Sultanates. - Kalckhoff, Franz: Die PreaBischen TelegrsphenMarken. - Krötzech, Hugo: Hirechfeld'eche Entwürfo und Druckproben zu den Sächeiecher Postfreimarken. - Lindenberg, Carl: Die Briefhogen von Britisch. Oetiadien. - Moers, J. B. : Niederlündieche Everis. Ohrt, Paul: Die Homburg'schen Steindruckmarkex $1 \%$ u. $2 \%$ Schilling der ungezähdten Ausgabe $186 \mathrm{t}$. - Rosenkranz, A.: Schleswig-Holeteinieche Posta. weisungen. - Sachse, Paul: Was dann? - Stadthagen: Mittbeilungen üher philatelietioche Prozesse. - Wsseermsnn, 0. u. Heinrich Fränkel: Rumänien, Auegaben 1866-1872. - Westoby, W. A. S.: Vom Gummi gebläat, - Mitglieder-Verzeichnio.

Ton, Hadbuch.
Berlin. (Berliner Philatelisten-Klub) Festschuift zur Feier des 25 jährigen Bestehens des Berliner Philatelisten Klub E. V. Im Auftrage des Kilubs hrsg. von Dr. F. Kalckloff, Dr. P. Pirl, Dr. F. Stenger. Mit 11 Taf. u. 138 'I'extillustrationen. Berlin: Selbstverl. des B. Ph.-Kl. 1913. V111, 324 S. $8^{\circ}$.

Inhalt: Kalckboff, Frsaz u. Eduard Sechse: Geschichte des Berliner Philsteliaten-Klube. - Duerst, Georg B.: Die Eessis von Rumäuien. - Friedliader, Punl: Bsssaher - llanciau, Louis: Die Diligencias ron Uruguay. - Herrmann, A dolf : Duuckdaten dar Postksrteu von Wirttemberg. - Hofmann, Fmll: Hannover. Durchatich. - Pauli, Emil : Uber ded s1raf. rechtlichen Schutz der Postwartzeichen in Btlgien gegen Fillecbung und MiBbrach im Lichto der Philatelie. - Prrl, Paul: 1)er 3 und 20 Pf.-Weit von Helgoland im Orig. u. Neudr. - leichenhelm, Franz: Finrichtung der Markeubogen Frankreichs. - Sachs, Fraz: Die Nienetganzsachen Neuseelends, - Sschs, Franz: Ein Verglejch der Preise der moBgebendea Katsloge. - Sommerfeldt, Willy: Die sls Dipnetformulsre verwendeten iiherdruckten Krenzer-Postkarten von Wiirtlemberz. - Stenger, Erich: Essais u. Proben der Brief-, Porto-, Telegraphen- u. Stempelmsrken von Bsyern, - Zennig, Karl: Die KommunePost von 1871. - Kslckhoff, Franz: Einrichtungen u. Statistik des Berliner Philatalietea-Klubs.

- Katalog der Bibliothek des Berliner Phila. telisten-Klub E.V. Berlin: Verlag des B.Ph.-KI. 1908. $64 \mathrm{~S}, 8^{\circ}$.

- (Deutscher Verein fïr Philatelie in Berlin.) Vorträge über Postwerthzeichen. [Berlin: Selbstver], 1881.] $40 \mathrm{~S} .8^{\circ}$. [Als Ms. gedr.].

Inhs1t: Cohn, David: Die Postwernhzeichen vou Quensland. - Fouré, Georges: T'her die im Verkehr goweseneu Postmarken uyd gestempelten Freischeine voo Preussea. - Fouré Georges: Nachrichtea über Porte de Mar-Markeu, Mexiko. - Moens, J. B.: Die Pootmsrken von Wirttesuherg.

- (Internationale Postwertzeichen-A usstellung.)

- Elster, J.: Katalog der auf der luternationalen Postwertzeichen-Ausstellung zu Berlin 1904 aufgelegten Sammlungen von Fabrikbesitzer J. E. Berlin 1904.

- Katalog der Internationalen Postwertzeichen-Ausstellung Berlin vom 25. August bis 4. September 1904. I1rsg. von dem Ausstellungskomitee. Berlin 1904. $101 \mathrm{~S} .8^{\circ}$.

- Koch, G.: Kiatalog der auf der Internationalen Postwertzejchen-Ausstellung zu Berlin 1904 aufgelegten Sammlungen ron G. K., GieBen.

- (Tönighiche Bibliothek.) Rommelsche PhilatelistischeSammlung. Fehlliste. Berlin NIV.7: Königliche Bibliothek 1916. $32 \mathrm{~S} .4^{\circ}$.

- (Reichspostmuseum.) Beck, Carl: Das Reichspostmuseum u. seine Markenbestände. Berlin 1908.

- - Führer durch die Sammlungen des ReichsPostmuseums in Berlin. Berlin: Julius Springer 1888. $44 \mathrm{~S}$. и. Grundrib. $8^{0}$.

- - 2. Aufl. Berlin. Julius Springer 189l. 44 S. u. Grundrib. 80.

Katalog des Postmuseums im Kaiserl. Generalpostamt zu Berlin. Berlin: Selbstverl. $1878.52 \mathrm{~S} .8^{\circ}$

- Katalog des Reichs-Postmuseums. Im Auftrage des Reichs-Postamts bearb. von H. Theinert. Berlin: Julius Springer 1889. 372 S. $8^{\circ}$. 
Berlin.(Reichspostmuseum.) liatalog des ReichsPostmuseums Berlin: Julius Springer 1897. $576 \mathrm{~S} .8^{\circ}$

- Lindenberg, C.: Katalog der MarkenSammlung des Reichs-Postmuseums. Berlin 1888.

Stephan, Heinrich v.: Frgänzung der Geschichte der Correspondenzkarten. Berlin 1865.

13erliner Jriefmarken-Kalalog s. Kosack, Philipp.

Berliner Plhilatelisten-Klub. s. Berlin.

Bern. (Briefmarken-Tausch-Klub, Bern.) Bericht über die Briefmarken-Ausstellung jugendlicher Sammler, veranstaltet vom B. T.-K., B. am 21. Okt, 1906. Auszug aus dem Bericht der Tätigkeit des Preisgerichtes. Bern 1906. $10 \% .2 \%$. [Schreibmaschinenschrift.]

Bernard, Léon: 1901-1902. Preis-Courant der Marken derfranzösischen Colonien. Specialität meines Hauses. Paris [1901]. 16 S. 8\%.

- 1901-1902. En-Gros-Preisliste der Marken der französischen Colonien. Specialität meines Hauses. Paris [1901]. 4 S. 80.

- Preisliste 1903. Briefmarken der Französischen Folonien u. Postämter. Paris [1902]. 23 S. $8^{\circ}$.

- 2. Berichtigungsliste. Paris, April [1903]. 4 S. $8^{0}$.

- 6. Aufl. 1904-1905. Paris [1904]. 28 S. $8^{0}$.

Beschoren, Paul: Die Postwerthzeichen Sachsens. Referat des Herrn P. B. aus München. Vorgetragen von Herrn Gustav Reitz von Bollheim am Club-Abende des 19. Nov. 1880. Wien: Sigmund Friedl [1881], 4 S. m. Abb. $8^{\circ}$. [Verfassertitelirrtümlich, Bschoren"aufgedr.]

Bestelmeyer, A.: Preisverzeichniss über einzelne Briefmarken, ganze Couverts, Streifbänder u. Postkarten sowie über BriefmarkenAlbums. Hamburg [1890], 11, 17 S. $8 \%$.

Beyfuss, C.: Neuestes Verzeichnis aller bis jetzt ausgegebenen in- und ausländischen Briefmarken mit genauer Beschreibung derselben, zsgest. von C. B. Hannover: Georg Wedelkind 1863. 68 S. $8^{\circ} .\left(12^{\circ}\right.$.)

- 2. Auff. Dresden 1865.

Bibliographien philatelistischer Literatur. s. Beck, Carl; Fränkel, Heinrich; Gottschatk, Albert; König, Ludwig: Krasemann, Rudolf; Mittelmann, H.; Rommel, Otto; Suppantschitsch, Victor; Thalmann, Friedrich Wilhelm; Ton, Max.

Bibliothek, Plilatelistische. $8^{\circ}$. Bd.1. Moschkau, Alfred: Die Wasserzeichen auf den seit 1818 bis dato emittierten Briefmarken und Couverts . . Dresden 1871.

- - s. a. Moschkau, Alfred: Geschichte der Briefmarken und der Philatelie <Briefmarkenkunde〉. . . 3.-4. Autl. Leipzig 1878-80.

- Bd. 2. Moschkau, Alfred: Handbuch für Essais-Sammler. Leipzig 1875.
Jibliothek, Plilatelistische. Bd.3. Moschkau, Alfred: Handbuch für Postmarken Sammler... 2.-6. Auflage. Leipzig 1876-88.

- Bd. 4. Moschkau, Alfred: Zur Geschiclite der Philatelie... Leipzig 1879.

- Bd. 5. Kloss, Paul: Vereinigte Staaten von Amerika . . Leipzig 1880.

- Bd.6. Perlep, A.: Katalog der Stempelmarken aller Staaten. Leipzig 1880.

Bibliothek fiir Postwerthzeichen-Sammler. Bd. 2. Senf, Louis: Handbuch für PostkartenSammler . . Leipzig $1890[-91]$.

- Bd. 4. Kausch, Oskar: Die Sprachwissenschaft in der Briefmarkenkunde. "2. Aut. Leiprig 1891.

[Bd. 1 u. 3, sowie Fortsetz. der Bibliulhek sind uicht eracbienen.]

Biluliotluek fiir Sammler. $8^{\circ}$. Bd. 1. Brendicke, Hans: Einführung in die Kunde von den Postwerthzeichen. Berlin 1889.

[Bd. 2 u. ff, entha]ten nichts Philatelistisches.]

[]3illig, B.]: Unter dem Zeichen des Verkehrs. [Eine Biographie Dr. Ernst Heinrich Wilhelm von Stephans.] Julius Springer, Berlin 1895. 228 S. $8^{\circ}$.

Biographien bedoutender Philatelisten u. Postbeamter. s. Dewez, Freiherr von; Glasewald, Arthur Ernst; Gündel, Karl von; Haas, Theodor; Hermann, Emanuel; Hill Rowland; Kloss, Paul; Krause, Richard; Lindenberg, Carl; Moschkau, Alfred; Schmückert, Generalpostdirektor; Schulze, Adolf; Stephan, Heinrich v.; Suppantschitsch, Victor; Wende, Georg; Wilhelm, J.

Birubach, II.: Preisliste über sämmtliche Post. Werthzeichen von Rumänien, nebst einem Preisverzeichnisse ỉber Europa und überseeische Raritäten der Special-Briefmarkenhandiung H. B. Bukarest: Selbstverl. 1898. 36 S. $8^{\circ} .\left(16^{\circ}.\right)$

Blïtter, Fliegende aus Senfs Illustr. Briefmarken-Journal s. Senf, Gebr.

Blauhuth, Berulıard: Briefmarken Preis-Katalog. Leipzig: Selbstrerlag [1886]. $92 \mathrm{~S} .8^{0}$.

- Über die hauptsächlichsten Kennzeichen der Bremer Marken mit besonderer Rücksicht auf die besten Hamburger Fälschungen. Vortrag in der Sektion Leipzig ron B. B (SeparatAbdr. aus "Der Phitatelist" 1893 Nr. 7.) Dresden: Internationaler Philatelisten-Verein 1893. 8 S. m. Abb. $8^{\circ}$.

Bochmann, Eugen von: Die Postmarken des russischen Reiches und deren Entwertung. Mit Abb.von Postwerthzeichen, W Vasserzeichen u. Entwerthungsstem peln. Nach eigenen Untersuchungen $u$, amtlichen Quellen von E. von B. (Sonderabdr. aus: Ill. Briefmarken-Zeitung 1892 No. 5 u. ff.). Leipzig: Ernst Heitmann 1892. 40 S. m. Abb. $4^{0}$.

- Die Postmarken des russischen Kaiserreiches. Leipzig: Hugo Krötzsch 1895. 178 S. u. 21 'Taf. 80. (Krötzsch: Permanentes Beibuch mit Lichtdrucktafeln T. 3.) 
Bochman u, lingen von: Dic Schweizer Marken der Emission 1854-63. (Sonderabzug aus der Festschrift des Berliner Philatelisten-Klubs 1898.) Berlin: Selbstverlag des Klubs 1898. $32 \mathrm{~S} .4^{\circ}$.

- - s. a. Berlin (Berliner Philatelisten-Klub). in: Festschrift zur Feier des zehnjährigen Bostehens des B. Ph.-Kl. 1898.

Bö̈thius: Einiges über das Brief-u. Verkehı-swesen im Alterthum; in: Senf, Gebr., Taschenbuch für Briefmarken-Sammler 1889.

Bollheim, Gustar Reitz Eller ron. s. Reitz Edler von Bollheim, Gustav.

Borek, Richard: Die Briefmarken der Italienischen Postämter in der Levante. 〈Olınc Berücksichtigung der Briffmarken für Albanien.) Braunscluweig: Richard Borek 1908. 2 S. u. 1 Taf. $4^{\circ}$.

- Die Kreta-Marken mit dem "Ellas"-Aufdruck in Buchdruck. Braunschweig: Richard Borel 1909. 4 S. u. 1 Taf. m. Abb. $4^{\circ}$.

- Neuheiten Preisliste. s. Abt. "Zeitschriften".

Börresen, M.: Preisliste über skandinavische, isländische u. finnische Postwerthzeichen. Drammen (Norwegen): Selbstverlag Novbr. 1890. $19 \mathrm{~S} .8^{\circ}$.

- Dezbr. 1890. 19 S. [nebst] Nachtr. 2 S. 80. - 1893. 20 S. $8^{\circ}$.

Bosnien 1. Herzegowina. Kohl, Paul: Illustr. Katalog der Freimarken von Europa Abt. 5. "Bosnien".

- Kropf, H.: Die Postwertzeichen der Üsterr.ungar. Monarchie. Prag 1902 v. 1908.

- Neulinger, Eduard von: Special- Katalog sümmtlicher Brief- u. Zeitungsmarken von Österreich-Ungarn. Abt. d., Bosnien $\mathrm{u}$. Herzegowina. Wien 1888.

- Sauer, K. Cl.: Permanenter BriefmarkenKatalog „Bosnien". Mährisch-Ostrau [1889].

Braud, Herrmaun: hatalog aller seit 1840 bis einschlieBlich 1882 erschienenen Briefmarken, Couverts, Postkarten, Streifbänder etc. nehst Verkaufspreisen. Hrsg. von H. B. Naumburg a. d. S.: Selbstverl. 1882. 92 S. $8^{\circ}$.

Brasilien. Bruck, A.: Illustrierter Katalog iiber Brasilianische Postwertzeichen. (Preis. liste $\mathrm{Nr} .151894$ ) Rio de Janeiro 1894.

Braunschweig. Berger, L.: Die Postwerthzeichen des Ilerzogthums Braunschweig. Braunschweig 1893.

- Lindenberg, C.: Die Briefumschläge von Braunschweig. Berlin 1892.

- Moschkau, Alfred: Zur Geschichte der Philatelie. Leipzig 1879. Artikel: ${ }_{\pi}$ Eine Curiosität von Braunschweig*.

- Rommel, O. đ Krötzsch, H.: Braunschweig. s. Krötzsch, H.: Permanentes Beibuch mit Lichtdrucktafeln. . . Absehnitt V. Leipzig $1893-96$.

- Verein für Briefmarkenkunde Frankfurt a.M.: Der Durchstich einer Braunschweiger Marke. Frankfurt a. 11. 1891.
Braunselıreig. ( Cremania-Ring", Verband Deutscher Postwertzeichensammler-Vereine.) Der XIV. Germania-Ringtag. (Separatdr. aus der "Berliner Briefmarken-Zeitung" vom 15. 8. 1908.) Braunschweig 1908. 6. . 8\%.

Braunschweig, Johannes: IIändlerliste über 100 Adressen enthaltend. Lïbeck 1872.

Brauswetter, Rudolf: Allerlei Wissenswertes über Furopa-Mlarken. Fin Wfgweiser für Philatelisten. (Sonderabdruck aus der ${ }_{n}$ Berliner Briefmarken-Zeitung ${ }^{*}$ 1913, H. 7-10.) Berlin: Philipp Kosack \& Co. 1913. 24 S. 80.

Breimeier, Wilhelm: Die Postwerthzeichen ron Gibraltar seit 1889. Fine Fortsetzung del Lindenbergschen Bearbeitung im "Großen Handbuch der Philatelie*. (Extrabeilage zur "I]lustr. Briefmasken Keitung" 1892. Nr. 8.) Leipzig: Frnst Heitmann 1892. 16 S. m. Abb. $8^{\circ}$.

Bremeu. Blauhuth, B.: Ueber die Lauptsächlichsten Kennzeichen der Bremer IJarken. Dresden 1893.

- Lindenberg, C.: Die Briefumschläge von Hamburg und Bremen. Berlin 1894.

- Rommel, O. \& II. Krötzsch: Bremen. s. Krötzsch, H.: Permanentes Beibuch mit Lichtdrucktafeln... Abschnitt VI. Leipzig 1893-96.

Brendicke, Hans: Der Briefmarken-Sammler. Hrsg. von der Redaktion des Guten Kameraden. Mit 168 Abb. Stuttgart, Berlin, Leipzig: Union, Deutsche Verlagsgesellschaft 1900. $131 \mathrm{~S} .8^{\circ}$.

(Illustrierte Taschenbächer für die Jugend Bd. 11.)

- - 7. neu bearbeitete Aufl. Ebrla. [1908] 135 S. m. 193 Abb. 8\%.

- - 13.-15. Tausend. Ebda. 1914. 138 S. m. $221 \mathrm{Abb} .8^{\circ}$.

- Enführung in die Kunde von den Postwerthzeichen. Eine Anleitung für angehende und erfabrenere Briefmarken-Fammler. Berlin: Sigmar Mehring 1889. II, $112 \mathrm{~S}$. m. 3 Taf. Abb. $8^{\circ}$.

(Bibliothek fiir Sammler Bd. 1.)

- Ist die Philatelie Wissenschaft oder nur Manie? in : Wenzel, E.: Philatelistisches Nachschlagebuch.

- Die Ludwig Reederschen Sammlungen zur Geschichte des Postwesens. Denkschrift überreicht zur Feier des 10. Stiftungsfestes des Berliner Pbilatelisten-Klubs am 23. Jan. 1898. Berlin 1898. $31 \mathrm{~S} .4^{\circ}$.

Breslan. Zechmeyer, Georg: Der Briefmarkenbandel vor den Breslaner Gerichten. Nürnber.g 1888 .

Briefmarken sind keine Kleinigkeit. s. Avenarius, Ferd.

Briefmarkenbilderbogen. s. Oelimigke \& liemschneider.

Briefmarken-B̈̈rse Pilzecker. s. Pilzecker, 0.

Briefmarkenfrenul, Der. s. Ruhl, Moritz.

- s. a. Storch, Adolf Fr. 
Briefuarken-IIandbucll s. Kohl, Paul: Großer Katalog u. Briefmarken-Handbuch.

Briefmarkenlandel, Der, vor den Breslauer Gerichten s. Joseph Dr. u. Zechmeyer, G.

Briefmarkenhandlung des Literarischen Musoums ill Leiprig s. Zschiesche, Katalog [Zehnte Aufi. I883].

Briefmarkenhandluugen aller Welt theile unt Länder s. Heinhold, J.

Briefmarken-Katalog, Berliner. s, Kosack, Philipp.

Jriefmarkenkönig, Der. s. Beauregard, George de $u$. H. de Giorsse.

Briefmarken - Normal - Katalog, Illustrierter, 1910. Chemnitz: Paul Kohl (1909) XX, 943 S. $8^{0}$.

- 1911. Fbda. (1910) XXV1, 981 S. m. Abb. Leinw. geb 8n.

- 1912. Ebda, (1911) XXXV111, 1024 S. m. Abb. Leinw. geb. $8^{\circ}$.

- 1913. Ebda. (1912.) XXXVIII, 1072S. m.Abb. Leinw, geb. $8^{\circ}$.

Fortsetz. s.u.d. T. „Nermal Katalog, Neuer, für Briefmarken".

Briefmarlien-Reform-Katalog. s, Kohl, Paul.

Briefmarkensammeln. Vom Briefmarkensammeln. s. Daheim-Kalender.

- Balke, Franz: Sollen Schüler Briefmarken sammeln? GöBnitz S.A. 1908.

- Scholtze, Carl, Joh.: Prakt. Anleitung zum Briefmarkensammeln. Berlin 1900.

- s. a. u. Hand-und Lehbücher der Philatelie.

Briefmarken-Sammler, Der s. Withalm, Hans.

Briefmarken-Sammler u. JriefmarkenhandIungen aller Weltteile u. Liinder. s. Heinhold, J.

Briefmarkentableau mit 260 Abbildungen u. statistischen Notizen. Wien: Administration der "Post" 1872. gr. $2^{\circ}$.

Briefmarken-Tauseh-Klub Bern. s. Bern.

Briefmarken-Versteigerungen. Kohl, Paul: [Katalog Nr. 1 einer] Sammlung von Briefmarken Furopa und Amerika. Auktion in München in der Galerie Helbing am 11. bis 15. Novemb. 1913 ... Chemnitz, IVeststr. 18: Paul Kohl G. w. b. H. u. München, Wagmüllerstr. 15: Galerie Helbing 19I3. IV, 124 S. m. Abb.i. Text. $8^{\circ}$.

- - [Katalog Nr. 2 einer] Samm]ung von Briefmarken Afrika-Australien [nur] Europa <einschl. Alt-Deutschland) [enthaltend]. Auktion in München in der Galerie Helbing am 11. bis 14. Februar 1914. Chemnitz u. München: Ebda. 1914. IV, 92 S. m. Abb. i. Text. $8^{\circ}$.

- - [Katalog Nr. 3 einer] Sammlung Deutsclie Kolonien und Postämter. Briefmarken-Auktion in Nünchen in der Galerie Helbing am 20. bis 22. April 1914. Chemnitz u. Mïnchen: Ebda. 1914. IV, 38 S. m. Abb.j. Text. $8^{\circ}$.

- [Kata]og Nr. 4 einer] Sammlung Europa, Alt ] yeutschland und Ausland. BriefmarkenAuktion in München in der Galerie Helbing am 25. u. 26. Juni 19I4. Chemnitz u. München: Fbda. 1915. IV, 83 S, m. Abb. i.'Text. $8^{\circ}$.
Briefmarken-Versteigerungen. Kiohl, TauI: [Kataleg Nr. 5] Friedrichsche Prachtsammlung und anderer Besitz. Unfassend Europa, Alt.Deutschland und Ausland. BriefmarkenAuktion in München in der Galerie Helbing am 25. u. 26. Juni 1915. Chemnitz u. München : Ebda. 1915. ]V, 73 S, m. Abb, i. 'Text. $8^{\circ}$.

Maier, M. Kurt: [Katalog der] BriefmarkenVersteigerung im Architektenhaus zu Berlin, WilbelmstraBe $92-93$ am 25 u. 26. September (1916). [Nebst:] Kunstdruck-Beilage. Berlin: M. Kurt Maier. 1916. 40 S. u. 26 Taf. m. Markenabb. Kart. m. Seidenschnur. Quer $8^{\circ}$. [Enthält nur "Altdeutschland".]

- s. a. Auktions-Kataloge.

Briefmarken-Zeitung fiir die.Jugend s. Moschkau, Alfred.

Briefnarken-Zeitungs-Kataloge. Nittelmann, 11.: Katalog der existierenden BriefmarkenZeitungen. Mährisch-Ostrau 1889.

- Internationaler Philatelistischer ZeitungsKatalog 1910. Leipzig (1909).

Briefunstıläge. s. Couverte.

- s. a. u. d. Eiuzelstaaten.

Brief-u. Verkelırswesen imAltertum s,Bö̈thius.

Britiseh-Ostindien. Lindenberg, C.: Die Briefbogen von Britisch-Ostindien. Berlin 1898.

l3ruck, Alfons: Illustrierter Katalog ïber lirasilianische Postwerthzeichen. Rio de Janeiro: Selbstverlag 1894. $15 \mathrm{~S} .8^{\circ}$.

131'uder, Willy, [d. i. Willy Dauth]: Erstes philatelistisches WeltadreBbuch in Taschenformat. Redigiert von W. B. Frankfurt a. Main: H. J. Dauth [1896]. $64 \mathrm{~S}, 8^{\circ}\left(12^{\circ}\right)$.

Brummer, Leon: Die Ba rerischen Portomarken s. Berlin. (Berliner Philatelisten-Klub.) in: Festschrift zur Feier des zehnjährigen Bestehens des B. Ph.-Kl. Berlin 1898.

Jrunner, J.: Das Postwesen in Bayern in seiner geschichtlichen Entwicklung von den Anfängen bis zur Gegenwart. München: Hermann Lukaschik 1900. 244 S. m. Abb.u. ] Bildn. $8^{\circ}$.

Brums, Johannes: Das Postwesen, seine Entwickelung und Bedeutung. Leipzig: B. G. Teubner 1907. 134 S. 80. (Aus Natur und Geisteswelt Bd. 165.)

Bsehoren. s. "Beschoren".

Bucl, Das, der Sammlungen. s. Klasing, $O$.

- - von der Weltpost. s. Veredarius.

- neue, von der Weltpost. s. Schweiger Lerchenfeld.

- - schwarze, der Philatelie \&. Lietzow, P.

IBuchara. Schüller, Fr.: Die persische Post u. die Postwerthzeichen von Persien u. Buchara. Linz a. D. 1893.

Bulgarien. Antoniades 1892-98. Katalog über orientalische Postwertzeichen. Constantinope] 1892.

- Beschreibender Katalog sämtlicher orientalischer Postwerthzeichen 〈deutsclı u.französ.) ConstantinopeI 1893. 
Bulgarieu. [Glasewald, A. F.]: Neues u. Vergessenes über Bulgarien v. Mumelien. Vortrag geh. auf dem $\mathrm{X}$. Deutschen Philatelistentag in GöBnitz, S.-A. 1898.

- Mruby, R.: Katalog aller türkischen, ost. rumelischen, bulgarischeu u. russischen Lovantepostmarken, Karten $u$. Couverts mit l'reisen. Constantinopel 1884-1891.

- Katalog über orientalische Postwerthzeichen. Constantinopel 1887.

- Marcus, M. H.: Katalog u. Preisliste orientalischer Postwerthzeichen. Constantinopel 1896.

Bund Deutscher nnd Österreiehiseher Philatelisten-Vereine. B. D. u. Ö. Ph.-V. gegründet auf dem 8 . doutschen PhilatelistenTage zu Köln a. Rh. im Juni 1896. 46 Vereine mit 5673 Mitgliedern. Mannheim 1898. $32 \mathrm{~S} .8^{\circ}$.

- Bundesbuch. Ausg. 1901. Hrsg. von der Bundesleitung. Ebda. 190I. 56 S. $8^{\circ}$.

- Mitglieder-Verzeichnis v. Satzungen des Bundes Deutscher u. Österreichischer Philatelisten-Vereine. Gegr. Juli 1896. (Nebst Bundes-Adressen u. Hinweise auf die BundesFinrichtungen.) Frankfurt a. 11.1907. 19 S. 80.

- s. a. Deutscher Philatelistentag. Bericht über den X. - X XV. Deutschen Philatelistentag . . . $1898-1913$.

Bundestag des Bundes Deutscher u. Österreichischer Philatelisten-Vereine. s. Deutscher Philatelistentag: Stenograph. Berichte u. Protokolle 1899-1913.

Canada. s. Dauth, H. J.: Abdruck postamtlicher Documente H. 1.

Caspart, Rudolf : Ist die Ausgabe von Jubiläumsmarken tatsächlich vorteilhaft? \&. Deutscher Philatelistentag; in: Die Vorträge vom XXII. D. Ph. . . . in Kiel am 14. August 1910.

- Liechtenstein. Vortrag. s. Deutscher Philatelistentag: in: Stenograph. Bericht des XXIV. D. Ph. ... zu Marktredwitz 2.-6. August 1912.

- Fin philatelistischer Streifzug durch die südamerikanischen Unabbängigkeitskriege. s. Deutscher Philatelistentag; in Stenograph. Bericht des XXIII. D. Ph.... zu Wien 8.-12. Septbr. 1911.

- Über systematisches Typensammeln (mit kleiner Ausstellung während des Vortrags). s. Deutscher Philatelistentag; in Stenegraph. Bericht des XXI. D. Ph.... zu Karlsbad 19.-24. August 1909.

Castle, M. P.: Die selteneren Marken Grobbritanniens. s. Berlin (Berliner Philatelisten-Klub) in: Festschrift z. Feier des zehnjührigen Bestehens des B.Ph.-Ǩ. Berlin 1898.

Ceylon. Staehle, Emil: Katalog und Preisliste der Postwertzeichen Ceylons. Mannheim 1896.

Chalmers, Patriek: Zur Beleuchtung der Frage über die Adhesiven Briefmarken. Zuschrift an die Mitglieder der Internationalen Phila- telistischen Gesellschaft zu Dresten. London: Effingham Wilson and Co. 1890, $12 \mathrm{~S} .8^{\circ}$.

Chalmers, Patriek: Zur Beleuchtung der Frage über die Adhesif-Briefmarken. Offener lirief an die Mitglieder der Internationalen Philatelistischen Gesellschaft zu Dresden. Verbess. Ueberselz. London: Fffingham WVilson and Co. $1890.12 \mathrm{~S} .8^{\circ}$.

- Ueber die Frfindung der Briefmarken. Wimbledon: Selbstrerl. Septbr. 1890. 1 Bl. $4^{\circ}$.

- Ueber die Erfindung der Briefmarke. Verbesserte Uebersetz. Wimbledon: Selbstver"l. Oktbr. 1890. $1 \mathrm{Bl} .4^{\circ}$.

Chalmers-11ill-Streitfragc, Die. Wimbledon: Patrick Chalmers 1891. $2 \mathrm{Bl} .8^{\circ}$.

[Mehr alo 104 Pamphlete u. Einzolblätter, daron 9 Flugschriften in deutecher Sprache.]

- s. a. Chalmers, Patrick: 'Zur Beleuchtung der Frage über die Adhesiven Bliefmarken.

Evans, Edward Benjamin; Die Chalmer's-Hill-Frage.

Gündel, Carl von: Die Chalmers-Iillsche Streitfrige.

Kloss, Paul: Streitfrage „Chalmers coutra Hill".

Chelius, A.: Der Specialsammler von Bayern nach Abstempelungen. München: Selbstverl. 1900. 11, 29 S. $8^{\circ}$.

China. Andersch, M.: Die deutsche Post in der Türkei, in China $u$, in Marokko ... Berlin 1912.

- Chinesische Couverte; in: Moschkau, A., Zur Geschichte der Philatelie.

- [Friedemann, Albert]: Die Aushülfsmarken von Tsingtau u. ibre Fälschungen. Leipzig 1903.

- Kleeberg, Paul: China, seine Post u. seine Freimarken von 1878-1910. Chemnitz 1911.

- Katalog der Marken der Kaiserlichen Post in China. Chemnitz 1911.

- Maus, J.: Die Post in China. Vortrag gehaiten auf d. XIII. Deutsch. Philatelistentag zu Berlin 1901.

- Rommel, Otto: Die chinesischen Lokalmarken. Budapest 1907. (Leipzig 1906.)

- Die chinesischen Privatpostmarken. Würishofen 1911.

Clirewy: Die Anfertigung der Briefmarken in der Reichsdruckerei zu Berlin; in: Isakovics, A. v.: Internationales Tausch-, Adreb-u. Hülfsbuch für den Tauschverkehr T. 2.

Clausius, W. T. s. Bauschke, Gustav: Katalog aller bekannten seit 1840 bis Mitte 1865 emittierten Briefmarken.

Clericus, L.: Ein Beitrag zur Geschichte des alten Postwesens; in: Senf, Gebr.: Taschen. buch f. Briefmarken.Sammler 1889.

Cohn, David: Die Postwerthzeichen von Queensland s. Berlin (Deutscher Verein für Philatelie in Berlin) in: Vorträge uber Postwertzeichen [1881]. 
Coldewe, 0.: Katalog ïber alle bekannten, seit 1840 his Dezember 1869 ausgegebenen Briefmarken, die zu den beigefügten Preisen durch $\mathrm{O}$. C. in $\mathrm{W}$ olfenbüttel bezogen werden können ... Wolfeubüttel: Selbstverlag 1870. 48 S. $8^{\circ}$.

Cöln a. Rh, Denkschrift zur Eröffnung des Reichs-Postgebäudes an den Dominikanern in Cöln (Rhein) Novbr. 1893. Cöln: J. G. Schmitz in Komm. 1893. 139 S. m. Abb., I FaksimiléTafel, Lichtdr.-Titel u. 3 Grundrissen. $8^{\circ}$.

Columbia s. Kolumbien.

Commune Marken, Pariser. in : Moschkau, Alfred: Zur Geschichte der Philatelie.

- Zennig, Karl: Die Kommune-Post von 1871. Berlin 1913.

Connell-Marke, Die, in: Moschkau, Alfred: Zur Geschichte der Philatelie.

Correspondenzkarten. Finke, Georg: Geschichte des Penny-Porto-Systems u. der Briefmarken. "Anhang:" Úber die Erfindung der Postkarte.

- Friedl, Sigmund: Illustr. Katalog sämmtl. bis Anfang 1878 erschienenen Postkarten, Anweisungen, Mandate, Nacbnahmen, Postfrachtbriefe etc. Wien 1878.

- Gündel, Carl ven: Die Postkarte, ihre Erfindung v. ibre Verbreitung; in: Senf, Gebr.: Taschenbuch für Briefmarken-Sammler 1889.

- Haas, Theodor: Handbuch sämtl.Postkarten, Kartenbriefe, Postanweisungen u. Paket-Begleitadressen. Leipzig (1891).

- Herrmann, Emanuel: Die Correspondenzkarte. Halle a. S. 1876.

- Kalckhoff, Franz: Die Frfiudung der Postkarte u. die Korrespendenzkarten der Norddeutschen Bundespost. Leipzig 1911.

- Iíloss, Paul: Verzeichnis u. Beschreibung aller Postkarten mit aufgedrucktem Werth. stempel nobst Nachtr. Jresden 1880.

- Lariscb, Anselm: Postkarten-Katalog, enth. sämtliche bis 1890 erschienenen Postkarten. München 1891.

- Moschkau, Alfred: Photograph. Souvenir f. Deutschlands Philatelisten, B1.3. Correspondenzkarten-Tableau. Nossen 1875.

- Senf, Louis: Handbuch für PostkartenSammler. Leipzig 1890[-91.]

- Stephau, Heinrich : Ergänzung der Geschichte der Correspondenzkarten. Berlin 1865.

Converte. Friedl, Sigmund: lllustr. Katalog sämmtl. bis Anfangs 1878 erschienenen Briefumscbläge, Streifbänder etc. IVien 1878.

- Moschkau, Alfrod: Photographisches Souvenir für Deutschlands Philatelisten. Bl. 5. Couvert-Tableau. Nossen 1875.

- - Zur Geschichte der Philatelie. Artikel: „Das Sammeln ganzer Couverte". Leipzig 1879.

- Prietsch, F. B.: Catalog aller his April 1875 emittirten Postcouverte. Leipzig 1875.
Crole, B. E.: Geschichte der deutschen Post von ihren Anfängen bis zur Gegenwart. Dem deutschen Volke erzählt und geinen Post. beamten gewidmet von B. W. C. Leipzig: W. Malende [1889], $479 \mathrm{~S} .8^{\circ}$.

-_ - Zweite unveränderte Aufl. Ebda. 1892. 479 S. $8^{\circ}$.

- Illustrierte Geschichte der deutschen Post von ihren Anfängen bis zum Ableben Kaiser Wilhelms I., bez. bis zum Tode Stephans. Ein Volksbuch für Jedermann, insbesondere für Verkehrsbeamte, Philatelisten, Freunde der Geschichte u. des Verkehrswesens u.s.w. von Bruno Emil König. Mit vielen in den Text gedr. Abb. sowie Notenbeil., Bilderund Briefmarkentafeln. 3. bed. verm. u. verbess. Aufl. Bd. 1. Geschichte der hrandenburgisch-preußischen und der norddeutschen Post bis zu deren Entfaltung zur deutschen Reichspost, sowie deren ersten Kntwicklungsphasen n. der Entstehung des Weltpostvereins bis zum Ableben Stephans. Berlin u. Leipzig: Friedrich Luckhardt (1900). XIV, 372 S. $8^{\circ}$. Leinw. Geb.

[Bd. 2 ist nicht erechionon.]

Crome-Scliwieniug, C.: Fritz der Sammler. Eine Geschichte für die Jugend. Leipzig: Hugo Krrötzsch 1899. 45 S. m. Titelbildn. u. Briefmarkenabb. $8^{\circ}$.

- Über Presse und Philatelie. s. Deutscher Philatelistentag; in: Stenograph. Protokoll über die Sitzung des XI. Deutschen Philatelistentages am 29. Juli 1899 ...

CuJa. Friederich, Rudolf: $Y^{1 / 4}$. Fine Studie. s. Berlin (Berliner Philatelisten-Klub) in: Festschrift zur Feier des zehnjährigen Bestehens. Berlin 1898.

- s. a. Spanien.

laheim-Kalender für das Deutsche Reich. Auf das Gemeinjahr 1907. Biclefeld v. Leipzig: Velhagen \& Klasing 1907. IV, 288 S. $8^{\circ}$.

Inhalt: S. 178-196: Vom Briefmarken6ammeln. Pereöulichee u. Sachlichee, Leid u. Freud. Von einem altou Sammler.

1)änemark. Kohl, Paul: Illustr. Katalog der Freimarken von Europa. Abt.4. Dänemark ... Chemnitz 1895.

- Rijse, O. V.: Dänemark-Studie. MIünchen 1893.

- Ruben, M. F.: Vollständige Preisliste von Dänemark u.s.w. Kopenhagen 1889/90.

Dänisch-Westindien. Ruben, M. E.: Vollständige Preisliste von ... ${ }_{n}$ Dänisch-Westindien". Kopenhagen 1889/90.

Danuenfelser, W. F.: Katalog über alle bekannten seit 1840 bis April 1864 ausgegebenen Briefmarken, die zu den beigefügten Preisen durch F. IV. Dannenfelser in Leipzig bezegen werden können. Leipzig: Dannenfelser 1864. 27 S. $8^{\circ} \cdot\left(32^{\circ}\right)$

[s. a. Iditeraricches Huseum unter gleichlautendem Titel gedr.]

batenzeiger d. Weltpostgeschichte. s. Schranka, Eduard Maria. 
1)autlı, 11. J.: Catalogue de l'Union Postale Universelle. Abdruck postamtlicher Documente. Hrsg. von H. J. D. Frankfurt a. M. H. J. Dauth [1890]. $8^{\circ}$.

H. 1. Ägypten, Brasilien, Canada, Luxemburg, Neuseeland, Norwegen, Schweden, St. Pierre. $31 \mathrm{~S}$.

H. 2. Inbalt S. 33-44: A ufstellung sämmtlicber Postwerthzeichen Perus. Ǘbersetzung der uns von der General-Postdirection zum Ablruck ïberlassenen Aufstellung der Société Philatélique zu Lima. Ebda. 1890. [Unvollendet.]

- Katalog altdeutscher Postwerthzeichen. Frankfurt a. M.: H. J. Dauth 1890. 14 S. $4^{\circ}$.

[ө. a. "Freudenetein ${ }^{4}$ unter gleichlautendem Titel ged ruckt.]

- Manco-Liste zu Ferd. Meyer's Katalog mit Angabe von ca. 6000 Preisen für BriefmarkenSammler. Hrsg. von H. J. D. Frankfurt a. Main: Gebrïder Knauer 1884. 56 S. $8^{\circ}$.

- Nachtr. Ebda. 1885. $16 \mathrm{~S} .8^{\circ}$.

- Manco-Listen für Briefmarken-Sammler. Hrsg. von H. J. D. Ausg. 1. Zu Zschiesche's illustr. Briefmarkenalbum. Ebda.1884. 76 S.80.

- - Ausg. 2. Zu Schwaneberger's Briefmarken-Sammelbuch ... Ebda.1884. 96 S. $8^{0}$.

- Ausg. 3. Zu Schaubek's illustr. Briefmarken-Album ... E Ebda. 1884. 86 S. $8^{\circ}$.

- s. a. Mangold, Otto: Catalogue de l'Union Postale Universelle. Abdruck von den General-Postämtern überlassener Scbriftsti̊cke.

Dencker, Dr. jur.: Die Abarten u. Farbenunterschiede der Deutschen Adler-Kolonialmarken. s. Deutscher Philatelistentag; in: Stenograph. Bericht ii. d. XV. Deutschen Philatelisten-Tag. ... in Pforzheim 1903.

1)eroce0, E.: Geschichte der Postwertzeichen von Serbien 1866-1911. Nach amtlichen Quellen. (Durchgesehener u. ergänzter Sonderabdr. aus der Berliner Bliefmarken-Zeitung 1911 u. dem Ganzsachensammler 1912/13.) Berlin: Philipp Kosack \& Co. 1914. 89 S. m. Abb. $4^{\circ}$.

Deutselı Kolonien. Andersch, M.: Die deutsche Fost in der Türkei, in China und in Marokko... Berlin 19I2.

- Brendicke, Hans: Einiges aus den deutschen Kolonien. Vortrag. s. Deutscher Pbilatelistentag; in: Stenograph. Bericht ü. d. XIII. Deutschen Philatelisten.Tag zu Berlin 1901.

- Demcker, Dr. jur.: Die Abarten u. Farbenuntersehiede der Deutschen. Adler-Kolonialmarken. Vortrag gehalten auf dem XV. Deutschen Philatelisten-Tag ... in Pforzhein 1903.

- Dister, G.: Das Vineta-Provisorium. Hannover 1907.

- Fregse, Gustav: Preis-Bucb "Europa" mit Anh. "Deutsche Folonien", Februar 1905. Hannover 1905.
Deutsehe Kolonien. [F'riedemann, Allert]: Die Aushülfsmarken von 'T'singtau u. ihre Fälschungen. Leipzig 1903.

- Friedemann, Albert: Dic Postfreimarken u. die Fntwertungen der deutschen Postanstalten in den Scliutzgebieten und im Auslande. Leipzig 1908.

- Kalckhoff, Franz: Die Postkarten der deutschen Schutzgebiete u. der deutschen Postanstalten im Auslande. Leipzig 1902.

- Knopf (-Goldberg), Dr.: Entwurf zur Anlage einer deutschen Schiffs- u. Marinepoststempel-Sammlung. GöBnitz 190I.

- Kublmann, K. J.: Deutschland und Kolonien (Marken u. Ganzsachen). Spezial-Kata$\log \mathrm{Nr}, 1$. Hannover [1901].

- Metzkes, Ernst: Spezial-Sammlung der Marken des Deutschen Reiches, seiner Auslandposten u. Kolonien. Barmen [1912].

- Reichspostamt. Post- u. Telegraphen-Nachrichten für den Verkehr mit den Deutschen Schutzgebieten. Berlin 1913.

- Schmidt (-Schwerin), Max: Verzeichnis der Kaiserl. Deutschen Marine-Schiffsposten. GöBnitz, S.-A. 1903.

- (Senf, Gebrüder): Illustr. Spezial-Katalog der deutschen Kolonialmarken und der Deutschen Postämter im Auslande. Leipzig 1904.

- Z Zweite Aufl. Leipzig 1907.

- Siebelist, Otto: Die Post im Auslande. 2. Aufl. Berlin 1892.

- 4. Aufl. Berlin 1909

Deutscher Philatelistentag. (Stenographisehe Berichte u. Protokolle.) Bericht über den am 18. August 1889 zu Mainz in der Stadtballe abgehaltenen Ersten Deutschen Pbilatelistentag. Nach stenographischen Aufzeichnungen im Auftrage des leitenden. Vereins für Briefmarkenkunde" Mainz hearb. von G. Sandkuhl, Kastel a. Rh. Mainz: Verein fül Briefmarkenkunde Mainz I. (1889). 7 S. $8^{\circ}$.

- - über den am 9. u. 10. August $1890 \mathrm{ab}$ gebaltenen 11. Deutschen Philatelisten-Tag zu Frankfurt a. M. Frankfurt a. M.: Higentum des Vereins f. Briefmarkenkunde Frankfurt a. M. (1890). $72 \mathrm{~S} \cdot 8^{\circ}$.

Die Verhandlungen enthalten fulgende Anträge in Form wienenschaftlicher Abhandlungen: Antrag 1. Weegmann. E. : Stellungaahme gegen den Hamiltonochen Vertrag mit den Centralamerikaniechen Repu. bliken. - Antrag 2. Glaeewald, A. E.: Wie känuen die Ssmmler gründlicher ale hieher var Betrïgern u. Falschern gewarnt u. geochüizt werden? - Antrag 3. Schindler, A.: Gründung einee Centralarganes für die Gesammtinteresen der Briefmarkenkunde. - Antrag 4. Thier, Mrsx: Gegen das Auevchneidon ron Ganzeachen. - Antrag 5. Schindler, A.: Uber die willkürliche Preiseteigerung vou Patwerthzeicben, namentlich der altdeutechen. - Antrag 6. Kloes, Paul: Streitfrage ${ }_{n}$ Chalmere contra Hill".

- - über den am 20., 21. u. 22. Juni 1891 abgehaltenen 11I. Deutschen Philatelisten'Tag zu Dresden. Dresden: Internationaler Philatelisten-Verein 1891.26 S. $8^{\circ}$.

["Wiesenechaftl. Teil enthält keine eigentlichen Varträge, eondern nur Beratungen.] 
Dentscher Philatelistentag. (Stenographische Berichte u. Protokolle.) Bericht ïber den IV. Deutschen Philatelistentag, abgehalten in Prag vom 9.-11. Juli 1892. (Prag 1892.) $61 \mathrm{~S} .8^{\circ}$. Wisenschaftl. Teil ${ }^{\mu}$ enthält den Vartrag: Töpfer, J. Ad.: Garantiestempel.

- Stenographischer Bericht ïber den 5. Deutschen Philatelisten-Tag in Berlin am 2., 3. u. 4. September 1893. Berlin 1893.68 S. $8^{\circ}$.

Wiscenechaftl. Toil " enthalt folgonde Vorträge: Kalckhoff, Franz: Úber die Einteilung der Marken u. ïber den Sammelwert der einzelven Gattungen. Ein Beitrag zur Albumfrage. - Neidhard, Otto, G.: Üher den Wert deo Sammelne ron Marken auf Brief. Haas, Theodor: Uther die sngeblichen bayriechen Neudrucke, - König, Ludwig : Bericht über philstelist. Veröffentlichungen in Tageazeitnngen u. Familienjournalea. - Brendicke, Hans : Zweck u. Finrichtung von Briefmarkenbörsen, - Lindenberg, Carl: Über die Postwertzeichen-Sammlungen des Reich6-Postmugeums.

über die Verbandlungen des 6. Deutschen

Philatelistentages in Kiel am 15. น. 16. Juli 1894. Kiel 1894. 95 S. $8^{\circ}$.

Wissenschaftl. Tei]" enthält falgende Varträge: Brendicke, Hane: Die Zukunft der Philatelie. Kalckhoff, 'Franz: Über Markencaneervirung. - Lin. denherg, Carl: Die Postwerthzeichen Helgolsnde. Brummer, Leon: Über die hayriechen Neudracke.

- - des VII. Deutschen Philatelistentages zu Mannhoim am 14. u. 15. Juli 1895. Mannheim (1895). $78 \mathrm{~S} .8^{0}$.

Wisenschaftl. Teil enthält folgende Vortrkge: Glacewald, A. E.: Wio verbessern wir uneere Tauochverbindungen? - Motz, H.: Mißgtände in der Philatelio. - Brnmmer, L.: Die Philatelie im Dienste rer Spekplation. - Rieigari, L.: Die Marken der italieniochen Kleinstasten. - Rosenherg, A.: Die Postwertzeichen Helgalande.

- - des VIII. Deutschen Philatelistentages am 20.-22. Juni 1896 zu Köln a. Kh. Köln (1896). $99+(2)$ S. $8^{\circ}$.

[Enthält keine wissenschaftlichen Vortrăge, sondern nur die Beratungen zweck 8 Gründngg eme日 Bundes Deutecher u. Öeterreichiecher Philatelisten-Vereine.]

- des 9. Deutschen Philatelistentages in Hamburg am 25. u. 26. Juli 1897. Hamburg (1897). 52 , III S. $8^{\circ}$.

Wisenechaftl. Teil ${ }^{4}$ enthält folgende Vorträge: Micheleen, G.: UUber die Postwertbzeichen der Vereinigten Staaten von Columbien. - Krötzech, IJugo: Die Prüfung vou Pastwerthzeichen $\mathbf{n}$, deren Entwerthungen. - Laвeau, Jul.: Hamburg [вeine Post- n. seine Paotwerthzeichen].

- Bericht über den $X$. Deutschen Philatelistentag verbunden mit dem II. Bundestag Deutscher u. Österreichischer Philatelisten-Vereine zu GöBnitz S.-A. am 16.-18. Juli 1898. GöBnitz, S.-A. Oktober 1898. 30 S. $8^{\circ}$ [nebst]: Fä]schungstafel m. 10 Marken-u. Couvertausschnitt-Fälschungen aus einem vom "Deutschen Philatelisten - Verband GöBnitz S.-A." aufgehobenen Fälschungsuest.

${ }_{n}$ Wissenschaf thicher Teil" enthălt nur einen kurzen Vermerk über die gebaltenen Vorträge: Glacewald, A. E.: Neuer u. Vergessenes iber Bulgarien u. Rumelien. [Als Sonderdruok erschienen.] - Crame-Schwiening, C.: Die Tagespreser u. die Philstelie.

- Stenographisches Protokoll über die Sitzung des Xl. Deutschen Philatelistentages am 23. Juli 1899 im Königlichen Belvedère auf der Brïhl'schen' Terrasse, sowie Bericht über den XI. Deutschen Philatelistentag u. III. Bundes. tag Deutscher u.Österreichischer Philatelisten-
Vereine in Dresten 21. his 25. Juli 1899. Dresden (1899), 18 S. 8\%. [Nebst Anhang]: Leyde, Oscal: Ias Sammeln von Postwerthzeichen nach Abstempelungen. Vortrag. $3 \mathrm{~S}$. - Maus, J.: Die Schiffspost auf dem Boden. see. Voltrag. 9 S. u. 4 Lichtdrucktaf. Crome-Schwiening, C.: Über Presse und Philatelie. Vortrag. $2 \mathrm{~S}$.

Deutscher l'hilatelistentag. (Stenographische Berichte u. Protokolle.) Stenographisches Protokoll über die Sitzung des III. Deut. schen Philatelistentages am 29. u.30.Juli 1900 im Gesellschaftshause des Palmengartens 〈JagdschloB auf dem Niederwald in Frankfurt a. 11. Frankfurt a. M. (1900). 14 S. $8^{\circ}$.

Eutbält fnlgende wiseenschaftliche Vorträge: Glasewald, A. Fa. : Die Zukunft unserer Tausch vereinlgungen. - Crome-Schwiening, C.: Phllatelistisches Allerlei.

Stenographischer Bericht ïber den XIII. Deutschen Philatelisten-Tag zu Berlin, am 7.-10. Septbr. 1901. Berlin 1901. 32 S. $8^{\circ}$.

Wisenschaftl. Teil enthält folgende Vorträge : Msu6, J.: Die Post in China. - König, Ludwig. Uber Vereinsweren. - Brendicke, Н.: Einiges aus den deutbchen Kolonien. - Sellechapp, W.: Gebraucht u. ungebraucht.

über den X1V. Deutschen PhilatelistenTag u. den VI. Bundestag des Bundes Deutscher u. Österreichischer Philatelisten-Vereine in Wien am 5. bis 9. Septbr. 1902. (Sonderbeil. zum, Vertraul. Korrespondenz-Blatt philatel.Vereine" Jg. XII. Nr.12. Dezbr. 1902.) Kíl 1902. $40 \mathrm{~S} .8^{\circ}$.

Wissenschaftl. Teil ${ }^{\alpha}$ enthält folgende Vorträge: Pallauech, Z. J.: Zur Reform der Neubeitenbesorgung. - Glasewsld, A. E.: Der Ballast in der Pbilatelie.

- uiber deu XV.Deutschen Philatelisten-Tag u. den VII. Bundestag des Bundes Deutscher u. Osterreichischer Philatelisten-Vereine in Pforzbeim, 28. August bis 1. Septbr. 1903. (Sonderbeil. zum "Vertraul. KorrespondenzBlatt philatel. Vereine" $\mathrm{Jg} . \mathrm{X} 111 . \mathrm{Nr} .10$. Oktbr. I903.) Kiel 1903. 58 S. $8^{\circ}$.

Wisenechaftl. Teil « enthält folgende Vorträge: Pauli, Emil : Die schwarzen Listen, Kataloge u. Bücher auf dem Gebiete der Philutelie im Lichte deo Rechts. - Demcker. Dr, jur.: Die Abarten und Farbenunterschiede der Dentschen Adler-Kolonialmarken, - Ohrt, Paul : Photugraphie u. Projektion für philatelietische Zwecke.

- üher den XVI. Deutschen Philatelisten-Tag u. den VIII. Bundestag des Bundes Deutscher u. Oesterreichischer Philatelisten-Vereine in Leipzig 〈u. Halle a. S.〉 vom 19.-23. August 1904. (Sonderheil. zum Vertraul. Korrespondenz-Blatt philatel. Vereine Jg. XV. Nr. 2. Februar 1905.) Leipzig 1905. 59 S. $8^{\circ}$.

Wiscenschaftl. Teil ${ }^{\star}$ enthält folgende Vorträge: Willadt, Carl: Island epeziell I Gildi. [Bemerkenowerte kurze Ausfïhrungen zu dem ursprünglich vor. gesehenen aber nicht gehaltenen Vortrag des Dr. Bergmann, Hsnnover]. - Haas, Thendor: Die Geschichtc uneerer großen Raritäten. - Schwaneberger, H.: Über ganze Bogen. - Setf, Richard: Die Druckverfahren bei Herstellung der Pootmarken.

- - über den XVII. Deutschen Philatelistentag u. den IX. Bundestag des Bundes Deutscher u. Oesterreichischer Philatelisten-Vereine in Regenshurg vom 21.-24. Juli 1905. (Sonderheil. zum „Vertraul. Korrespondenz-Blatt" philatel. 
Vereine Jg. XV. November I905.) Regensburg 1905. $71 \mathrm{~S} .8^{\circ}$.

"Wisecuscbafti. Teil ${ }^{\mu}$ enthält folgende Vorträgo: Glasewald, A. Fa : Postalisch verwondete Fälochungen,

- Köuig, Ludwig: Uber philatelietieche Literatur 〈mit Vorlagen〉.

Deutscher Philatelistentag. (Stenographische Berichte u. Protokolle.) Stenographischer Bericht über den XV111. Deutschen Phila. telistentag u. den $\mathrm{X}$. Bundestag des Bundes Deutscher v. Oesterreichischer PhilatelistenVeroiue in Nürnberg rom 31. August bis 2. Septbr. 1906. (Sonderbeil. zum Vertraul. Korrespondenz-Blatt philatel. Vereine Jg. 16. Novbr. 1906.) Nürnberg 1906. $64 \mathrm{~S} .8^{\circ}$.

Wiseenschaftl. Teil ${ }^{4}$ enthält folgende Vorträge: Willadt, C. : Nicbt-Katologieiertes aus den Gebiete der altientgcheo Staaten. - Willadt, C.: Uber Katalogproiso: - Schardey, Paul: Wohin ooll das ftihren? - Pauli, Emil: Entwertung falocher Marken. - Mis sou: Gescbichte der telephoniachea Marken ron Bel. gien.

- - des X1X. Deutschen Philatelisten-Tages und des XI. Bundes-Tages des Bundes Deutscher $u$. Österreichischer Philatelisten-Vereine zu Hamburg rom 23. bis 27. August 1907. Sonderabdr. aus dem Vertraul. Korrespondenz-Blatte philatelist. Vereine 1907, Nr. 9,10 u. 11.) Hamburg 1907. 68 S. $8^{\circ}$.

"Wiasenechaftl. Teil" entbält folgeude Vortrilge: Vicenz, Erast: Gibt es amtliobe Noudrucke u. Probe druck $\theta$ von Hamburger Steindruckmarken? - Sellechopp, W.: Western Franks, Freicouverte der Ameri. can Exprese-Compognied.

- - des XX. Deutschen Philatelisten-Tages u. des X11. Bundes-'Tages des Bundes Deutscher u. Oesterreichischer Philatelisten-Vereine zu GöBnitz (Sachsen-Altenburg) vom 1.-3. August 1908. GëBnitz 1908. [A. E. Glasewald in Komm.] $72 \mathrm{~S} .8^{\circ}$.

Die Vorträge im "Wissengchaftl. Toil ${ }^{*}$ sind auch besonders veröffeatlicbt. B. Doutscher Philatelistedtag: Vortrăge, gehalten auf dem XX. Deutgchen Philatclisten-Tage zu Gößaitz em 2. u. 3. Aug. 1908.

- - des XX1. Deutschen Philatelisten-Tages u. des X1II. Bundes-Tages des Bundes Deutscher u. Oesterreichischer Philatelisten-Vereine zu Karlsbad rom 19.-24. August 1909. (Sonderbeil.: zum Vertraul. KorrespondenzBlatte philatel. Vereine XX. Jg. Nr. 1. Januar 1910.) Hamburg 1910. 71 S. $8^{\circ}$.

Wiegengchaft]. Teil" entbält folgende Vorträge König Ludwig: Philatelistischer Bericht uher die Zeit vom XX. bia zum XXI. Deutscheu Pbilatelieten-Tage. - Pauli, Emil : Zweck u. Ziele, sowie Entwicklungegang der Wisenschaftlichen Gruppe. - Krötzsch, Hugo: Bericht iiber die Neudruckfrage. - Balke, Franz: Philatelistieche Jugenderziebung. - Katecha, Franz: Die Hellasaufdrucke auf dea Marken von Kreta, deren Fälschungen nnd Falscher, - Caspart, Rudolf: Uber вystematisches Typeneammeln (wit kleiner Austellung während des Vortrags).

- - des XXII. Deutschen Philatelisten-Tages u. des X1V. Bundes-Tages des Bundes Deutscher u. Oesterreichischer Philatelisten-Vereine zu Kiel vom 11. bis 16. August 1910. (Sonderbeil. zum Vertraul. KorrespondenzBlatte philatelistischer Vereine XX1.Jg. 1911.) [Hamburg 1911.] $56 \mathrm{~S} .8^{\circ}$.

Die Vorträge im "Wisseaschaftl. T'oil" aind beeon. ders veröffentlicht. 8. Dentecher Philatelietentag. Die Vorträge rom XXII. Doutschen Ptilateliatentage in Kiol am 14. Auguet 1910. beutscher Plijatelistentag. (Stenographische Berichte u. Protokolle.) Stenograplischer Bericht des XXIIl. Deutschen Philatelistentages und des XV. Bundestages des Bundes Deutscher u. Österreichischer Philatelistenvereine zu Wien vom 8. bis 12. Septbr. 1911. (Sonderbeil. zum Vertraul. KorrespondenzBlatte philatelistischer Vereine. XXII. Jg. 1912.) (Hamburg 1912.) 90 S. 8*.

Der Wisenschaftliche Teil “ entbält folgende Vorträge: Caspart, Rudolf: Ein philatelietiecher Streifzug durch die eüdamerikaaiøcben Unabbängigkeitgkriege. - Scheihe, Max : Mittel u. Wege, die Popularitht dee Briefmarkensammelns im Publikum zu fördern. - Woerz, Hone Ritter von: Ǔber die Neudrucke der Marken von Oaterreich u. der Lombardei.

- - des XIIV. Deutschen Philatelistentages u.des XV1. Bundestages des liundes Deutscher u. Osterreichischer Philatelistenvereine zu Marktredwitz vom 2. bis 6. August 1912. (Sonderbeil. zum Vertraul. KorrespondenzBlatte philatelistischer Vereine. XXIII. Jg. 1913.) (Hamburg 1913.) $76 \mathrm{~S} .8^{\circ}$.

Wisgenschaftl. Teil ${ }^{4}$ entbält folgende Vorträge: Caepart, Radolf: Liechtenetein. - Glasewald, A. E. Die Levante u. deren Poeten. [Auch ale Sonderár. erecbienen.]

- - des XXV. Deutschen Philatelistentages u. XV11. Bundestages des Bundes Deutscher u. Oesterreichischer Philatelistenvereine zu Hanuover vom 15, bis 16. August 1913. Hannover (1914). $4^{\circ}$ [in Schreibmaschinenschrift].

Wissenschaftlicher Teil" en thalt folgende Vortrăge : Glasewsld, A. E.: Dio bietoriecho Entwicklung der deutschen Postelempel. Unter Vorlage von Teilen einer Semmlung von Briefen deutscher Stasten von 1783-1850. [Auch ale Sonderdruck der PhilateliatenZeitung erechienen.] - Genth, F.: Wie $\theta$ o Ist, wie $\theta$ nicht iat und doch gein könnte!

bentscher Philatelistentag. (Sonderschriften.) Beck, Carl: Praktischer Wegweiser durch das Reich der Philatelie. Fin Notbehelf für jüngere Briefmarkensammler nebst einer Geschichte der Deutachen Philatelistentage. Berlin 1899.

- Geschichte und Geschäftsordnung der Deutschen Philatelisten-Tage, 1889-1908. Hanburg 1908. $16 \mathrm{~S} .8^{\circ}$.

- Pauli, Emil: Geschichte, Wesen u. Bedeutung der Deutschen Philatelisten-Tage. 1889 bis 1913. Berlin 1913.

- Vorträge, gehalten auf deu XX. Deutschen Philatelisten-Tage zu GöBnitz am 2. u. 3. August 1908. GëBnitz, S.-A.: A. F. Glasewald $1908.16 \mathrm{~S} .8^{\circ}$.

Inbalt: Balke, Fradz: Sollen Schüler Briefmarken eammeln? [Auch als Sonderabdr. veröfientlicbt 8. Bolke, Franz.] - Glasewald, A. E.: Reotbestände und deren Schickeale. - Hosa, Theod, Uber die Fohldrucke mit verkehrtem Mittelstick.

- Die Vorträge vom XXII. Deutschen Philatelistentage in Kiel am 14. August 1910. Hrsg. von der Wissenschaftlichen Gruppe des Ständigen Ausschusses der Deutschon Philatelistentage. Leipzig: Selbstverlag der W. G. (Huge Krötzsch in Komm.) 1910. 16 S. $4^{0}$.

In alt: Apstein, Dr.: Über Maschinenstempel. Caepart, Rudolf: Iot die Ausgabe von Jubiläumemarken tateächlich vorteilbaft? - Viceuz, Ernst; Dio Kunet im Bilde der Briofmarken. 
Deutscher Verein fiir Briefmarkenkunde Prag. s. Prag: Deutscher Verein f. Briefmarkenkunde Prag.

Dentsches Reich. Berger, H.: Illustr. Preiskatalog der Telegrafen- u. Stempelmarken der deutschen Einzelstaaten. Hamburg 1896.

- Crole, B. E.: Geschichte der deutschen Post von ihren Anfängen bis zur Gegenwart. Leipzig 1889 u. 1892.

- - Illustr. Geschichte der deutschen Post von iliren Anfüngen bis zum Ableben Kaiser Wiłlelms l., bez. bis zum Tode Stephans. Berlin u. Leipzig (1900).

- Dauth, H. J.: Katalog altdeutscher Postwerthzeichen. Frankfurt a. MI. 1890.

- Denkschrift zur Einweihung des neuen ReichsPostgebäudes in Goslar. Brannscliweig 1894.

- Denkschrift zur Eröffnung des Reichs-Postgebäudes an den Dominikanern in Cöln (Rhein). Novbr. 1893.

- Dieckmann, Carl: Postgesclichte dentscher Staaten seit einem halben Jahrtausend. Leip. zig 1896.

- Dieckmann, W.: Die dentschen Privatposten. Hannover 1898.

- Düsterbehn, प.: Katalog der deutschen Eisenbahnmarken. Oldenburg 1908.

- Eisenbahnmarken-Katalog Deutschland. Oldenburg 1911.

- Entwickelung, Die, des Post- n. Telegraphenwesens im Herzogthum Sachsen-Altenburg. Altenburg 1900.

- Erdmann, A. E.: Die deutschen Privat-Postwerthzeichen. Leipzig 1887.

- Faulhaber, B.: Geschichte der Post in Frankfurt a. Main. Frankfurt a. M. 1884.

- Frendenstein, S.: Katalog altdeutscher Postwerthzeichen. Leipzig 1890.

- Friedemann, Albert: Proken u. Entwürfe für die Marken der Dentschen Reichspost u. der Deutschen Schntzgehiete. Leipzig 1909.

- Gast, R.: Katalog altdeutscher Postwerthzeichen. Dresden 1890.

- Glasewald, A.E.: Permanenter BriefmarkenKatalog. Deutschland. Mährisch-Ostrau 1888.

- - Die historische Entwicklung der dent. schen Poststempel. GöBnitz 1913.

— - Katalog u. Mancoliste sämmtlicher... herausgegebenen deutschen Privatpost-Marken [u. Ganzsachen]. Gößnitz 1887 u. 1888.

- - Verzeichnis der in Gruppen auftretenden Nummern-, Orts- etc. Stempel der deutschen Einzelstaaten u. Kolonien. Gößnitz 1905 [nebst] Nachtr. 1906.

- Glasewald, A. E., O. Sattler \& Fr. Wagner: Handbuch der deutsehen Privatpostwerthzeichen, 2 'l'eile. Gößnitz 1889/90.

- Gobbert, Fr.: Verzeichnis sämmtlicher antlich ausgegebenen Briefmarken der Staaten des dentschen Bundes bis znr Errichtung des deutschen Reiches. Breslau 1891.

- Göpfert, R.: Staatspost und Privatpost. Dresden 1887.

- Grosse, Oskar: Die Beseitigung des Thurn- u. Taxisschen Postwesens in Deutschland durch Heinrich Stephan. Mlinden 1898.

Deutsches Reich. Grosse, R.: Das Postwesen in der Kurpfalz im 17. u. 18. Jahrhundert. Tübingen 1902.

- Herrmann, Fr.: Die Marken und Ganzsachen altdeutscher Staaten, nebst deren Preisen. Leipzig 1891 .

- Jung, J.: Entwickelung des deutschen Postu. Telegraphenwesens in den letzten $25 \mathrm{Jah}$ ren. Leipzig 1893.

- Kalckhoff, Franz: Die Telegraphenmarken u. Fernsprechscheiue der Deutschen Staaten. Berlin 1910.

- Kausch, Oskar: Die Nameukunde der Länder u. Städte des Dentschen Reiches. Leipzig 1890.

- Knust, Jul.: Illustr. Einkanfs-Ḱatalog über Altdeutsche Marken 1894/95. Harburg a. d. Elbe 1894.

- Koch, Georg: Katalog der auf der Internationalen Postwertzeichen-Ausstellung zu Berlin 1904 aufgelegten Sammlungen von G. K. GieBen.

Inbalt: Deutscher Reich, Altdeutgche Staaten, Ganze Bogen. Berlin 1904.

- Koh], Paul: Katalog der Freimarken Deutschlands. Chemnitz 1893.

- Illustr Katalog der Freimarken von Europa. Abt. 1. "Deutschland." Chemnitz 1894/95.

- Krötzsch, Hugo: lllustr. ausführlicher Katalog über dentsche Postfreimarken. Leipzig 1896.

- - Die Postfreimarken der Deutschen Staaten in: Krötzsch, Permanentes Handbuch der Postfreimarkenkunde. Bd. I. Abschnitt 1. (Rommel, Otto: Deutsche Reichspost.) Leipzig 1893 .

- Kuhlmann, K. J.: Deutschland und Kolonjen (Marken u. Ganzsachen). Spezial-Katalog Nr. I. Hannover [1901].

- Külutmann's Posthandbuch. Dresden 1913. [в. a. Stephan, Der kleine.] Dresden 1901.

- Lindau, Joseph: Katalog zur Sammlung der Postwerthzeichen Altdeutsche Staaten sowie Deutsches Reich mit Kolonien" in ungebrauchten Stücken. Heidelberg 1900.

- Maus, J.: Die Schiffspost auf dem Bodensee. Dresden [1899].

- Metzkes, Frnst: Spezial.Sammlung der Marken des Deutschen Reiches, seiner Auslandposten u. Kolonien. Barmen [1912].

- Niggl, Arthur: Das Postrecht. Stuttgart 1913 u. 1914.

- Pauli, Emil: Über den strafrechtlichen Schutz der Postwerthzeichen des Deutschen Reichspostgebietes gegen Fälschung u. MliBbrauch. Berlin 1911.

- Reichspostamt. Das Reichs-Postgebiet. Topographisch-Statistisches Handbuch für die Reichs-Post- u. Telegraphen-Anstalten Deutschlands. In zwei Teilen. Berlin 1878. $8^{\circ}$.

T. 1. VIII, $935 \mathrm{~S}$.

,2. $1 \mathrm{~V}, 263 \mathrm{~S}$. 
Deutsehes Reieh. Radenhausen, H.: Katalog der cleutschen Privatpostwerthzeichen. Mähr.Ostrau 1888.

- Reinheimer, A.: Katalog der deutschen Entwertungsarten von 1849-1875. 2 IIefte. Frankfurt a. M. 1891/92.

- 1llustr. Preiskatalog der deutscheu postalischen Entwertungsarten. Dresden 1894.

- Rommel,Otto: Deutsche Reichspost (Krötzsch, Handb. Bd. 1. Abschnitt 1.) Leipzig 1893.

- - Special-Catalog der Postwertzeicheu der Deutschen Postbezirke. Dresden 1896.

- Sauer, C. Cl.: Permanenter Katalag, „Deutschland".

- Schimmelpfennig, W.v.: Die bildlichen Dar. stellungen auf den deutschen Privatmarken in: Senf, Gebr.: Taschenb. f. BriefmarkenSammler pro 1889.

- Schindler, A.: Über die willkürliche Preissteigerung von Postwerthzeichen, namentlich der altdeutschen. Antrag 5 auf dem VII. Deutschen Philatelisten-Tag zu Frankfurt a. MI. 1890.

- Schreiber, J. J.: Stadtbriefbeförderung für 2 Pfennig. Berlin 1874

- Stenglein, M.: Die Post-, Bahn- u. Telegraphengesetzgebung des Deutschen Roiches. Berlin 1893.

- Stephan, Der kleine. Posthandbuch. Dresden 1901.

[a, a. Kühtmann'e Poetbaudbuch. Dreoden 1913.

- Sternheim, Carl: Katalog der deutschen Privatpostmarken. Schöneberg-Berlin 1902 [nebst Nachtr.] 1904.

- - 2. Aufl. Ebda. 1909.

- Tarif- und Beförderungs-Bestimmungen der Neuen Berliner Omnibus- u. PacketfahrtActien-Gesellschaft. Gegr. 1884. Berlin $1892 / 93$.

- Ton, Max: Altdeutsche Postwerlzeichen. kunde. Leipzig [1913].

- Verzeichnis der z. Z. bestehenden Privatpost.Unternehmuugen. [GöBnitz, S.-A., 1890.]

- Wagner, F.: Die Privatpost-Wertzeichen. H. 1. "Heidelberg." Berlin-Friedenau 1912.

- Wehner, Fr.: Über halbiert gebrauchte Marken des Deutschon Reichspostgebiets. Hamburg [1900].

- Willadt, Carl: Nicht-Katalogisiertes aus dem Gebiete der altdeutschen Staaten. Vortrag. s. Deutscher Philatelistentag; in: Stenograph. Bericht ü. d. XVIII. Deutschen Philatelistentag. . . in Nürnberg 1906.

- Wülbern, Arthur: Illustr. Preis-Katalog der Telegraphen- u. Stempelmarken Deutscher Staaten u. Kolonien. Hamburg 1912.

- Zschiesche, Alwin: Katalog sämtlicher his 1891 ausgegebenen Couverts, Postkarten $u$. Kreuzbandstreifen der deutschen Staaten. Naumburg a. S. 1891.

- s. a. Deutsche Kolonien; Feldpost; Postgebiihren; Post- u. Telegrammgebühren; Post-
gebïhren-Ordnung; Postgebiihren-T'ahelle; Post-Tarif; Privatposten, Deutsclio, u. P'rivatpostwertzeichen.

Deyhle, Robert: Illustrirter Philatelisten-Kalender pro 1881. Hrgg, von R. D. Bern: R. Deyhle \& Cie. 1881. 64 S. $8^{\circ}$.

In balt: Kalendarium. - Der achweizerische Ober. poetdirektor Edmund Höhn. - Glasewald, Frnet Der Briefträger oder unverbofteo Glïek, Novelle. - Dejble, Robert: Die Hungertour. Philatel. Reieerinnerung. - Aut einer Sammlung postaliecber Gegenstände. - Rundschau auf das Jabr 1880. - Folgen einer philatelistiechen Vereinigung, Humoreske. Deyhle, Robert: Plauderei. - Mobrere Gedichte. Miezellen.

- Illustrirter Philatelisten-Kalender pro 188.2. Neue Ausg.] Ebda. 1882. 64 S. $8^{\circ}$.

In b a It: Kolendarium. - Dae Schwein n. die Briefmarke Eine Fahel. - Fugen Borel, der Director dee internationalen Fontbureau. - Agatha, Novelette. - Ein Abenteuer im Schwarzwald. - Alt-Bundearath Näf, der Begründer des echweizeriechen Postwegeno. - Deyble, Robert: Auf hoher Firn. - Deyble, Ro. hert: Der gelbe Pechrogel oder die Neujahrofeier des Philateliten Schribs. - Die ungebrauchte u. gestempelte Briefmarke. Eine Fabel, - Mehrere Gedichte. - Miscellen.

Deyhle, Robert, c Co.: Preisliste über schweizerische Postwerthzeichen der Briefmarkenhandlung R. D. \& Cie. Bern, Februar 1888. 8 S. $8^{0}$

lewez, Freiherr von: Biographie, in : Jeran, 0, Wegweiser für Verkehrswertzeichen-Sammler.

Dieckmann, Carl: Postgeschichte deutscher Staaten seit einem halben Jahrtausend. Unter Berücksichtigung der Einführung der Freimarken. Besonders für Fachmänner u. Postwertzeichen-Sammler. Leipzig: Ernst Heitmann 1896. 368 S. m. Marken-Ahb. $8^{\circ}$.

Dieckmann, W.: Die deutschen Privatposten. Denkschrift im Auftr. der Freien Vereinigung deutscher Privatposten verfasst. Hannover 1898. 39 S. $8^{\circ}$.

[Als Ms. gedr.]

Diena, Emilio: Die 1-Bajocco-Marke des Kir chenstaates. s. Berlin (Berliner PhilatelistenǨlub) in: Festschrift z. Feier des zehnjährigen Bestehens des B. Ph.-Kl. Perlin 1898.

Diggelmann, Jacob: Die Fälschung von Sammlungsobjekten $u$. die strafrechtliche Bekämpfung derselben. Zürich: Art. Institut Orell FüBli 1916. 179 S. 80

[Behandelt auch die Briefmarken von philate]ist. u. jnrist. Stand punkte.]

Dister, Georg: Zur Neudruckfrage. Im Auftr. des Ring-Vereins "Frisia" in Wilhelmshaven verfaBt von G. D. Mit einem Nachwort der Schriftleitung der "Germania-Berichte". Hrsg. vom Germania-Ring. Essen, Juli 1909. 12 S. $8^{\circ}$.

Dister, $f_{*}:$ Das Vineta-Provisorium. Hannover: Gustav Freyse 1907. 15 S. m. Abb. u. 4 'Taf. nehst Nachtr. 3 S. u. 1 Taf. m. Abb. $4^{\circ}$.

Djurling, Hilmer u. Rudolf Krasemann: Die Postmarken von Schweden 1855-1905. Nach amtl. Quellen hrsg. von Sveriges FilatelistFörening $u$. für das Deutsche bearb. von Hilmer Djurling u. Rudolf Krasemann. Leipzig: Hugo Krötzsch \& Co. 1908. 116 S.m. Abb. $8^{\circ}$. (Krötzsch, Handb. Ausland-Bd. 2.) 
Doczkalik, F.: 1 Landbuch der Munieipal-Stempelmarken Italiens. Kaposvár: Selbstverlag 1911. 38 S. m. Abb. 8*. [Unvollendet.]

Douau - Dainpfsehiffahrts - (Aesellscliaft. s. Oesterreich-Ungarı.

boubletten. Die Verwerthung von Doubletten; in: Jeran, O.: Wegweiser für Verkehrswertllzeichen-Sammler (Philatelisten-Fibel).

Iresden. (Internationaler Philatelisten-Verein.) Deutscher Philatelistentag. Bericht ïber den am 20., 21. u. 22. Juni 1891 ahgehaltenen III. Deutschen Philatelisten-Tag zu Dresden. Eigenthum des I. Ph.-V. D. 1891.

- - Mitteilung, Vertrauliche, des Intern. Philatelisten-Vereins Dresden. s. Abt. "Zeit. schriften unter: Mitteilungen.

- Plotz, Ernst: Katalog der Bibliothek des Intern. Philatel.-Vereins Dresden. Dresden: I. Ph.-V. 1903.

[Als Ms. gedr.]

- ${ }_{\pi}$ Vertraulich", I. Ph. -V. D., Copie des "Sehwarzen Buches" Nr. 154. Dresden [1892]. 21 S. $8^{\circ}$.

- Verzeichnis u. Beschreibung aller Post. karten. Bearbeitet von Mitgliedern des lntermationalen Philatelisten-Vereins. Dresden: Alwiu Nieske 1879. 176 S. $8^{\circ}\left(16^{\circ}\right)$.

[Veröffentlicht in einzelnen Teilen ale Frgänzung zur Deutschen Briefmarken-Zeitung "Union" 1879 , azch Vervollgtondigung 1880 ale Einzel-Werk unter dem Namen des Bearbeitere "Dr. P. Klos" erechienen. ө. 3. и. Klone, Paul.]

Druekverfalien, Die, bei Herstellung der Postmarken. Vortrag, gehalten auf dem XVI. Deutsehen Philatelisten-Tag ... in Leipzig (u. Halle a/S.) 1904. s. Senf, Richard.

Duerst, G. B.: Die Essais von Rumänien. (Sonderdruck aus der Festschrift des Berliner Plilatelisten-Kilubs 1913.) Berlin : Selbstverlag des Klubs 1913. 18 S. $8^{\circ}$.

- s. a. Berlin (Berliner Philatelisten-Klub) in: Festschrift z. Feier des 25 jähr. Bestehens des B. Ph.-Kl. Berlin 1913.

Diisterbehn, H.: Katalog der deutschen Eisenbahnmarken. Oldenburg: Selbstverlag 1908. $64 \mathrm{~S} . \mathrm{m} . \mathrm{Abb}, 8^{\circ}$.

- Eisenbahnmarken-Katalog Dentschland. Oldenburg: Selbstverlag 1911. 93 S. $8^{\circ}$.

[Bildet die 2. Aufl. deo 1908 erechienenen Werkes: "Katalog der deutschen Eigenbahnmarken".]

Eekelmann, P. \& Co.: Illustrirter Postwertzeichen-Katalog. Enthaltend alle bis 1891 erschienenen Postmarken, postaliseh gebrauchten Stempelmarken, Briefumschläge, Postkarten, Kartenbriefe, Postanweisungen, Streifbänder, Packetbegleitadressen u. Rủckscheine einschlieblich Fehldrucke, Typen u. Neudrucke unter Berücksichtigung aller Verschiedenheiten, bezüglich Zähnungen, Wasserzeichen, Klappenstempel, Formate, Gummirungen etc. Dresden: P. Wclielmann \& Co. 189. Schmal $8^{\circ}$.

[Siehe a. Friedl, Sigmund ${ }^{*}$ u. ${ }_{n}$ Senf, Gebrider, nnter gleichlautendem Titel gedruckt.]
Finteilung der Marken u. ïber den Sammelwert der einzelnen Gattungen s. Kalckhoff, Franz. Vortrag, geh. auf dem 5. Deutschen Philatelistentag Berlin 1893.

Eisenbahmmarken s. Düsterbehn, $H$.

Eissen, Flanz, \& Cos: Catalog sämmtlicher bis Anfang 1879 erscbienenen österreich.-vngarischen Briefmarken, Couverts, Postkarten, Anweisungen u. Nachnahmskarteu, mit t'reis: angabe. Budapest: Selbstverl. [1879.] 10 S. $4^{0}$.

[Den gleichen Katalog gibt es auch mit dem überklebten Namen Sigmund Friedl $^{u}$.]

Elb, Ferdinand: Catalog nebst Preisliste mit Beschreibung iber alle seit Anbeginn der Ausgabe von Briefmarken ủberhaupt bis zum heutigen Tage ausgegebenen und projectirten Briefmarken und Stempel, entlialtend bis dato 2800 Nummern. Hrsg. unter Beihilfe u. Revision eines höheren Postheamten u. Benutzung authentischer, officieller u. privater Quellen. [Nebst:] Nachtrag. Dresden: Selbstverlag 1864.132 S. $8 \%$.

- Nachtr. 2. Ebda. 1866. $19 \mathrm{~S} .8^{\circ}$.

- Nachtr. 3. Ebda. 1867. 8 S. $8^{\circ}$.

Elsaß-Lothringen s. Norddeutscher Postbezirk.

Elster, Johannes: Katalog der auf der Internationalen Postwertzeichen-Ausstellung zu Berlin 1904 aufgelegten Sammlungen von Fahrikbesitzer J. E. Berlin: Selbstverlag 1904. 4 S. $8^{\circ}$.

Engliselue Kolonien. Kohl, Paul: Jllustr. Freimarken-Katalog: Englische Kolonien. Chemnitz $1898 / 99$.

Entwertungen. Friedemann, Albert: Die Postfreimarken $\mathrm{n}$. die Entwertungen der deutsehen Postanstalten in den Schutzgebieten u. im Auslande. Leipzig 1908.

- Glasewald, A. E.: Die historische Entwicklung der deutschen Poststempel. Gößnitz, S.-A. 1913

- Verzeichnis der in Gruppen auftretenden Nummern-, Orts-etc. Stempel der deutschen Einzelstaaten u. Kolonien. GöBnitz, S.-A.1905.

- Knopf (Goldberg), Dr.: Entwurf zur Anlage einer deutschen Schiffs- v. MarinepoststempelSammlung. GöBnitz, S.-A. 1901.

- Pauli, Emil: Entwertung falscher Marken. 8. Deutschen Philatelistentag; in: Stenograph. Bericht i.. d. XVIII. D. Pb. in Nürnberg 1906.

- Pirl, Paul: Ein Beitrag zur Abstempelungsfrage. Charlottenburg 1911.

- Priwe, Amtsrichter: Die deutschen Schiffsbrief-Entwertungen. Leipzig, Breslau 1915.

- Töpfer, J. Ad.: Garantiestempel. Vortrag, geh. auf dem IV. Deutseben Philatelisten. tag in Prag 1892.

Entwickelung der Posten vom Altertum bis zur Neuzeit s. Haass, F.

- des Post- und Telegraphenwesens im GroBherzogtum Baden 1874-1896. Karlsruhe: Ch. F. Mïller 1897. 35 S. u. 4 Karten. $4^{\circ}$ (Lex. $8^{\circ}$ ). 
Fintwicklung des l'ost- u. 'l'elegraplionwesens im Herzogtum Sachsen-Altenburg. Altenburg, S.-A. Schnuphase 1900. 23 S. m. J 'l'af. A bb. $4^{\circ}$.

- des deutschen Post- u. Telegraphenwesens s. Jung, .T.

Erdmann, 1. [d. i. Arthur Ernst Glasewald]: Die Postmarken ron Griechenland. Philate. listische Studie.

[1. Aufl. nar ale Artikel in Nr. 1 der Mitteilungen des Bayrischen Philatelisten-Vereine in Minchen Jenuer 1886 erschiczen ]

- 2. Aufl. Separatdr. auf Seidenpapier. GöBnitz, S.-A.: A. E. Glasewald. 1886.

- - 3. Aufl. Ebda. April 1886. 3. T. Text u. '2 Bl. (Narkenfelder). $4^{\circ}$.

- 4.-6. Aufl. Kbda. Juni u. Juli 1886 je 8 S., davon $2 \mathrm{~S}$. Text u. 2 Taf. $4^{\circ}$.

- 7. Aufl. GöBnitz, S.-A.: Buchhandlung Arthur Glasewald. 1887. 14 S., davon 2 S. 'l'ext u. 6 Taf. $4^{\circ}$.

- 8. Aufl. Ebda. 1889. 16 S., davon 2 S. 'l'ext u. 8 Taf. $4^{\circ}$.

- - 9. Auft. Französische* Ausg., übers. von Paul Stroehlin, Genf. Ebda. 1890. $20 \mathrm{~S}$., davon $2 \mathrm{~S}$. Text u. 9 Taf. $4^{0}$.

- - 10. Aufl. Euda. 1892. 16 S., davon $2 \mathrm{~S}$. Text u. 8 Taf. $4^{0}$.

- - 11. Aufl. Ausg. A. für ,Durchschnittssammler. Ebda. 1896. 14 S., davon 2 S. Text u. 6 Taf. $4^{\circ}$.

- - - Ausg. B. für ,Spezialeamm]er". Ebda. 1896. 14 S., davon 2 S. Text u. 12 Taf. $4^{\circ}$.

- - Ausg. C. Dasselbe lose auf "Carton". Ebda. 1896. $4^{\circ}$.

- 12. Aufl. Nachtr. zu "Ausg. B.". Fibda. 1903. Mit weiteren 6 Taf. (zus. 18 Taf.). $4^{\circ}$. [Die Griechenlend-Studie iet auch in den Katalegen von Kohl-u. Scott enthalten, also diese denach besrbeitet.]

- Die deutschen Privat-Postwertbzeichen, mit Markenabb.u.Städtewappen.(Album m. Text.) Leipzig: Ernst Heitmann 1887. $111 \mathrm{~S} .4^{0}$.

- Nachtr. Ebda. August 1888. 58 BI. $4^{0}$.

Erhaltung und Schönheit s. Flatterer.

Erinnerungen an 1870-71. s. Moschkau, Alfred: Photographisches Souvenir für Deutschlands Philatelisten. Bd. 1. Nossen 1875.

Esbach, Friedrich Carl: Taxis. Festschrift zur Silberhochzeit des Fürstenpaares von '1hurn u. 'Taxis am 15. Tuli 19I5. Regensburg: Verlagsanstalt vorm. G. J. Manz, A.-G. 1915. 30 S. $8^{\circ}$.

[Vnthält u. a. kurze Hinweise auf die Thnrn u. Taxisschen Pestgerechteame.]

Essais u. Probedrucke. Krause, Richard: Über Essais-Sammeln im Allgemeinen u. die deutsclien Levante-Essais. Brünn 183 i.

- Moschkau, A.: Handbuch für Essais-Sammler. Leipzig 1875.

- Reinheimer, A.: KurzgefaBte Beschreibung der Essays-Sammlung von Martin Schroeder. Leipzig 1903.

- s. a. u. d. Einzelstaaten.

Ton, Handbnelı.
Essliurer, (': : Das l'ostwesen in Ostfriesland in der Zeit von 1744-1806. Aurich: D. Friemann 1908. IV, 90 S. $8^{\circ}$.

Furopa. Freyse, Gustav: Preis-Buch ${ }_{\text {Furo. }}$ pac... Hannover 1905.

- Europa-Katalog. Hannover 1907-1910. Friedemann, Albert: Briefmarken-Preisbuch Europa 1908-1914. Leipzig 1907 bis 1913. GlasewaId, A. H.: Preisbuch [der Marken von] Europa. 1-11. Aufl. GöBnitz 1890-1904.

- liohl, Paul: Freimarken-Katalog 1900. Euıopa u. europäische Kolonien. 2 Bde. Chemnitz 1900

- 1llustr. Katalog der Freimarken von Europa. Chemnitz $1894 / 95$ u. 1897.

Kosack, Philipp: Berliner BriefmarkenKatalog. Huropa. Berlin 1912-1915.

- Künast, 1 .: Preisbuch [der Marken] ron Europa. Berlin 1894-1900.

- Michel, Hugo: Europa-Katalog. Apolda u. Veimar 1910-1916.

- Michels Mignon. Europa-Katalog. Weimar 1914-1916.

- Schultze, Joh. O.: Preisbuch [der Marken von Europa. Oelsnitz i. Erzgeb. 1896.

- Seelig, Cr.: Preisbuch [der Marken] von Europa. Stettin 1896.

Willadt, Carl \& Co.: Lager-Preisbuch Europa". Pforzheim, Baden 1907-1913.

Zschiesche, Alwin: Katalog über alle bis 1896 ausgegebenen Bricfmarken von Europa. Naumburg a. S. [1896].

Evans, Edward Benjamin: Die Chalmers-HillFrage. Übersetzung aus dem, Stamp Advertiser" von J. Siewert. (Extrabeilage zu "Der Philatelist" 1890, Nr. 11.) Dresden: internat. Philatel.-Verein 1890. $8^{\circ}$.

Ewen, Henry L'Estrange: Katalog u. Preisliste der Brief-u. Telegraphen-Marken von Grohbritannien, nehst Verzeichnis der Abstempelungen. Übers. von E. D. Frank, Frankfurt a. M. Offenbach a. M. 1895. Druck ron Will. Gerstung. 112 S. $8^{0}$.

[Denteche Ubers. der 4. Aufl. in engl. Sprache.]

Fabergé, A. s. Schmidt, C. \& A. Fabergé: Die Postwertzeichen der russischen Landschaftsämter.

Filscher-Schutzfrage. Glasewald, A. E.: Wie können die Sammler griudlicher als bisher vor Betrügern u. Fälschern gewarnt u. geschützt werden? Antrag 2 auf II. Deutsch. Philatelisten-Tag zu Frankfurt a. M. I890.

- Diggelmann, Jacob: Die Fälscliung von SammJungsobjekten u. die strafrechtliche Bekämpfung derselben. Zürich 1916.

Fỉischungen. Zum Fälschungswesen; iu : Moschkau, A., Zur Geschichte der Philatelie.

- Fournier, F.: Prix-Courant de la Maison F. F. Nr. 14-17. ('enève (Suisse) 1909, 1911, 1912 u. 1914. 
Fỉsehungeu. Fournier, F.: Welcher Unterschied bestelit zwischen einer Marken-Nachahmung (Eac-similé, in Farbe, Zähnung, Format, Abstempelung usw. dem Original äbnlich) und einer Markenfälschung? Festgabe für die Herren Delegierten zum Schweizer Philatelisten-'I'ag in Zürich, 17.- 18. Juni 1911. Genf: F. Fournier 1911. 7 S. $8^{\circ}$.

- Glasewald, A. E.: Postalisch verwendete Fälschungen. Vortrag gebalten auf dem XVII. Deutschen Philatelistentag in Regensburg 1905.

- Lietzow, Paul: Das schwarze Buch der Philatelie oder Neudruck und Fälschung von Postmarken u. Briefumschlägen. Berlin 1879. Pauli, Emil: Entwertung falscher Mlarlien. Hrwiderungs-Rede, gehalten am 2. Septur. 1906 auf dem XV1l1. Deutschen Pliilatelistentag in Niirnberg.

_. - Die schwarzen Listen, Kataloge u. Bücheı aul dem Gebiete der Philatelie in Lichte des Rechts. Greifswald 1903.

_ - Ï̈ber strafrechtlichen Schutz der Postwertzeichen in Belgien gegen Fälschung und MiBbrauel im lichte der Philatelie. Berlin 1913.

Über den strafrechtlichen Schutz der Postwertzeichen des Dentschen Reichspostgebietes gegen Fülschung u. MiBbrauch. Berlin 1911 .

- Tobler, Adolf: Der Kampf gegen das Fäischerwesen. Vortrag. Bern 1914.

- VerzeichniB der aus dem Leutzsch'schen Concurse herrïhrenden, vom Deutsch. Philatelisten-Verband erworbenen Eohl'schen Fälschungen ... GöBnitz, S.-A. 1898.

- s. a. Le Fac-Similé in: Abt. Keitschriften.

Fälsehungen und Nendrueke. Auszug aus dem Vertraulichen Korrespondenzblatt, Jg. 1/XV. 1891-1905. Hamburg: Verein für Briefmarkenkunde zu Hamburg von 1885. $8^{\circ}$.

Farblafelu fir Postwertzeichen-Sammler s. Germania-Handbücher Bd. 1.

Faulhaber, B.: Geschichte der Post in Franli. furt a. M. Frankturt a. M.: Völcker 1881. $256^{\circ} \mathrm{S} .8^{\circ}$.

Felldrueke, Haas, Theodor: Über die Fehl. drucke mit verkehrtem Mittelstück. Vortrag. GöBnitz, S.-A. 1908.

Fehllisten über deutsche philatelistische literatur.

Berlin (Königl. Bibliothek). Rommelsche Philatelistische Sammlung, Fehlliste. Berlin 1916.

- 'liffany, J. k.: FehJliste der Abtheiluug von Deutsche Werke. St. Louis, Mo. 1894.

Feldpost. Becker, Herm. Joseph: Das Postwesen im Saargebiet... [nebst] Anh.: Bedeutung u. Tätigkeit der Post im modernen Kriege. Saarbruicken 1915.

- Franke, P.: Aushunftsbuch über die amtlichen Feldpostbestimmungen ... Düsseldorf $19 ! 4$.
Feldpost. Glasewald, Arthur Ernst: Die Post im Kriege. Beitråge zur Geschichte der Feldpost. Gößnitz [1914/15].

Grosse, O.: Die deutsche lieldpost (Unterm Eisernen Kreuz 1914/15. H. 24.) Berlin (1915).

_- _- Die deutsche Feldpost im TTeltkriege. Braunschweig (1916).

Jaap, Carl: Postbuch für den Feld-u. KriegrsPost- v. T'elegrammverliehr. Berlin 1915.

- Keitel, Otto: Feldpost-Ratgeber beim Verschicken u. Terpacken von Feldpostsendungen . Jueipzig 1914.

Köuig, Fimil: Deutsclilands Feldpost. Ein Gedenkblatt a. dem Deutsch-Französisehen Iirieg 1870-71. Gera 1871.

Krötzsch, Hugo: Karte der deutschen Poststraßien in Frankreich 1870/71. Leipzig 1895.

- - Norddeutsclier Postbezirk 〈nebst Feldpost 1870/71). Leipzig 1895.

(Krötzseh, Handb. Bd. 11.)

- Mosch]iau, Alfred: Krinnerungen an 1870-71. Nossen 1875.

Müller, A.: Zusammenstellung der wichtig. sten Feldpost-Vorschiften ... Weinheim 1914.

- Postuann, T.: Feldpostdienstordnung u. Ausführungsbestimmungen, sowie die Feldpostvorschriften für den Postbetriebsdienst. Frankfurt a. M. 1910.

- Reielispostamt. Die Klagen über die Feldpost. Ber]in o. J. [1914].

- Merkblatt für Feldpostsendungen. Berlin 1915. Gedr. in der Reichsdruckerei.

- Sommerfeldt, WVilly: Die Feldpost-Ganzsachen der OPD. (Oberpostdirektion) StraBburg. Berlin [1915].

- Speiser, W.: Von der Feldpost zur Postreform. Leipzig 1915.

- Verkelır, Der, mit der Feldpost. Gießen 1914.

- Wiltz, Hermann: Die Feldpostdienstordnung nebst Ausfïhrungsbestimmungen in gedräugter Darstellung. Schwerin i. Mecklbg. 1911.

Feldpost, Die. Eine Sammlung der einschlï. gigen Vorschriften als wichtiger Behelf fur die Kriegszeit. [Sonderabdr. aus: „Kallina's Post-Tarifen 1915*.] Wien: Druckerei- u. Verlags-Aktiengesellscliaftvorm. R.von Waldheim 1915. $11 \mathrm{~S}$. $8^{0}$.

- K. u. k. Organisation der k. u. k. Feldpost. - Leitung des k. u. k. Feldpostdienstes in militärischer Beziehung. - Dienstvorsehrift f. das k. u. k. Feldpostpersonal. Bentitzung der k. u. k. Feldpost. - Feld. postbetrieh E-47. Wien: (Hof- a. Staatsdruckerei) 1913. 102 S. m. I Taf.

Feldpost-Kontrollouch. Mit Feldpost-Tarif, Adressennacliweis u. Raum für Notizen. Leipzig: Siegbert Schnurpfeil 1915. $16 \mathrm{~s} .8 \%$.

Feldpost-0roles für 1870-1873. $2^{\circ}$. 
Finke, ficorg: Geschichte des Penny-PortoSystems u. der Briefmarken. Nit einem An. hange über die Erfindung der Postkarto. Zum 50 jährigen .Tubiläum der Biriefmarke. Nlit einem Portrait J. Chalmers, dessen Briefmarken-Entwürfen u, einem Postwertzeichen-Entwurfe Charles Whitings. Leipzig: Ernst Heitmann 1890. 49 S. u. 1 Taf. m. Abb. $8^{\circ}$.

- Vademecum des Philatelisten. Qualienbrück: H. Neyer 1889. $65 \mathrm{~S} .8^{\circ}$.

- Katechismus des Briefmarkensammlers (zugleich 2. Aufl. vom „Vademecum des Philate(isten $\left.{ }^{2}\right)$. Bremen: Mlarbes 1891. 63 S. $8^{\circ}$.

- (zugleich 3. Aut. vom ${ }_{n}$ Vademecum des Philatelisten"). Bremen: Marbes 1901.62S. 8".

Finnland. Finnland-Couvert 1845, 10 liep. schwarz. Fälschung; in: Moschkau, A., Zur Geschichte der Philatelie.

- Granberg, R.: Die Ganzsachen von Finnland. Berlin 1903.

- Hauciau, L.: Die Postwertzeichen des Grobfürstentuins Finnland. Berlin 1909

- Helisch, L.: Die Postwerthzeichen von Finnlaud. Tien 1880.

- Helsingfors Frimärkssamlare Förening: Katalog über die Freimarken des Grokfürstentums Finland. 2, 3. ฉ. 4. Aufl. Helsingfors 1904,1908 u. 1913.

Jürgens, W.: Katalog-Preisliste alter Marken, Karten u. Briefumschläge ven Finnland und RuBland. Helsingfors 1875-1883 u. Reval 1884-1891.

liohl, Paul: lllustr. Katalog der Freimarken von Europa. Abt. 4.. "Fimnland."

- Michailoff, J.: Preisliste über Marlien, Briefumschläge, Karten etc. von Finland. IViborg 1891 .

- Rommel, Otto: Die Privat-, Eisenbahn-u Dampfschifismarken von Skandinavien u. Finnland. GöBnitz, S.-A. 1909.

- liuben, M. E.: Vollständige Preisliste von ... Finnland ... Kopenhagen $1889 / 90$.

Fischer, Lndwig: Die Zukunft der Briefmarkenkunde in: Senf's Taschenbuch f. Briefmarken-Sammler pro 1889.

(Flatterer:) Erhaltung und Schömheit. Eim ernstes IVort an alle Sammler u. an solche, die es werden wollen. (Sonderabrl?. aus der Berliner Briefmarken-Zeitusg.) Berlin: Philipp Kosack 1908. 12 S. mit Abb. 40.

Flcisclumann, 1.: s. Martinetz, J. A. Internationales Briefmarkenländler-Adrebuch. Wien 1877.

Flugschrift des Dürer-Bundes zur ästhetischen Kultur, Nr.61. Briefmarken sind keine Kleinigkeit. s. Avenarius, Ferdinand.

Fouré, Georges: Nachrichten über Porto de Mar-Marken, Mexiko. s. Berlin. (Deutscher Verein für Philatelie in Berlin) in: Vorträge ïber Postwerthzeichen. Berlın ISSI.
Foure, Ceorges: Über die im Verkehr gewesenen Postmarken u. gestempelten frejscheine von YreuBen. s. Berlin. (Deutscher" Verein $f$. Philatelie in lerlin) in: Vorträge iiber Postwerthzeichen. Berlin 1 $<5$ !

Fouruier, F.: Prix-Courant de la Maison l. F. Editeur' de l'ac-Similés d'anciens timbresposte... (Ancienne Maison L.-II. Mercier. fondée en 1891.) Nr. 14. 1909. Genève 〈Suisse〉 1909. 22 S. 8o.
Nr. 15. 1911. Ebda. 1911. 28 S. $8^{\circ}$
- Nr. 16. 1913. Ebda. 1913. 34 S. u. (I4 S.).
80.
- Nr. 17. 1914. Ebda. 1914.63 S. $8^{0}$.

[Sämtl. Ausg. mit teilwcise deutscliem Text ]

Frank, E. 1).: s. Ewen, Henry L'Estrange: Katalog u. Preisliste der Brief-u. 'l'elegraphenmarken von Grobbritannien. Offenbach a. II. 1895 .

rranke, P': Auskunftsbucl über die amtlichen Feldpostbestimmungen u. die gesetzlichen bestimmungen über die Versorgung der Hinterbliebenen der gefallenes oder an Wunden u. sonstigen Kriegsdienstheschïdigumgen gestorbener Teilnehmer am Kriege 1914. Diisselderf (Apollinarisstr. 30): P. Franke 1914. 15 s. 80 .

Frinkel, Heinrich: Chronologische Tabelle der deutschen philatelistischen Zeitschriften I863-1893. Berlin: Selbstverlag 1894. (I6) S. v. $1 \mathrm{Bl}$. Berichtigungen. $4^{\circ}$. [Einseitig bedr.]

- s. a. Berlin. (Berliner Philatelisten-Club.) Festschrift zur Feier des zehnjährigen $\mathrm{Be}$ stehens.

Fraukfurt a, M. Faulhaber, B.: Geschichte der Post in Frankfurt a. M. Frankfurt a. M. 1884.

- Verein für Briefmarkenkuude zu Frankfurt a. MI.) Der Durchstich einer Braunschweiger Marke. Broschüre zur Wahrung berechtigter Interessen in der Streitfrage Thier-Reisse gegen Hanson. Frankfurt a. II. 1891. 36 S. u. I Taf. m. Abb. $8^{0}$.

- Mitteilungen des $V$. für B. zu F. a. M. s. Abt. "Zeitschriften".

Frankreich. Hoffmann, F. A.: Frankreich u. Colonien (Preisliste). Paris 1897.

- Kohl, Paul: 1llustr. Katalog der Freimarken von Europa. Abt. 2. "Frankreich".

- Mossauer, J.: Mitteilungen iiber unbekannt gebliebene Französische Postwertzeichen aus den Jahren 1850-1854. Straßburg 1896.

- Peichenheim, Franz: Einrichtung der Markenbogen Frankreichs. Berlin 1913.

- Schaupmeier, C.: Neuer Katalog der Postwertzeichen Frankreichs u, seiner Kolonien von 1849-1893. Darmstadt [1894].

- Preiskatalog der frauzösischen Marken samt Colonien. Paris 1896.

Französische Kolonien. Bernard, Lẻon: 1901 bis 1902. Preis-Courant der Marken der französischen Kolonien. Paris [1901.]. 
Französische Kolonien. Bernard, Léon: 1901-1902. En-Gros.Preisliste der Marken der französischen Colonien. Paris [1901].

- Preisliste 1903. Briefmarken der Französischen Kolonien u. L'ostämter. Paris (1902), - 2. Berichtigungsliste. Paris, A pril [1903]. - 6. Aufl. 1904-1905. Paris [1904].

- Schaupmeier, C.: Neuer Katalog der PostWertzeichen der französischen Kolonien. Darmstadt 1893.

- s. a. Frankreich.

Frascati, Salvatore: Briefmarken-Preisliste 8. Abt. "Zeitschriften".

Frondenberg, Werner: "Matador." Preisgekrönte Arbeit zu dem Preisausschreiben des Ganzsachen-Sammlers 19I2. (Sonderdr. a. Berliner Briefm.-Zeitung 1913). Berlin: Phil. hosack \& Co. 1913. 4 S. $4 \%$.

Froudenstein, S.: Katalog altdeutscher Postwerthzeichen. Leipzig: Giebr. Senf 1891. 14 S. $8^{\circ}$.

[ө. a.: "Dauth" II. "Gast" unter dem gleichnamizen Titel gedruckt.]

Freyse, fustar: Preis-Buch Europa mit Anh. Deutsche Kolonien“, Februar 1905. SSonderbeil. zum Internationalen Briefmarken-Offertenblatt März u. April 1905. Hannover: G. Freyse 1905. 64 S. $8^{\circ}$.

- Europa-Katalog (1907). Hannover: Freyse 1907. 96 S. $8^{\circ}$.

_- [Neue Ausg.] (1908.) Ebda. 1908. 104S. $8^{\circ}$.

- 1909. Ebda. 1909.

- 1910. Ebda. 1910. 100 S. $8^{\circ}$.

Die Post in Albanien. Erschöpfend philatel. Abhandlg. nebst lehrreicher $\mathbf{u}$. interessanter Mitteilung über Land u. Leute, Sitten, Politik etc. m. ca. 500 Künstl.-1llustr. u. 1 geograph. Kartenskizze. Hannover: Selbstverl. Leinw. geb.

[ln Vorbereitung, eoll orat nach FriedenaschluB erecheinen.]

Freytag, Rudolf s. Rübsam, Josef $\&$ Rudolf Freytag: Postgeschichtl. Dokumente des Fürstl. Thurn u. Taxisschen Zentralarchivs zu Regensburg $\langle 1504-1909\rangle$.

Friedemann, Albert: Die Anshülfsmarken von Tsingtau und ihre Fälschungen unter besonderer Berücksichtigung der beim Kaiserl. Gericbt von Kiautschou stattgehabten Gerichtsverfahren. Mit $22 \mathrm{Abb}$. und 3 Kunstdruck-Beilagen. Leipzig: Gebr. Senf 1903. 56 S. $8^{0}$.

- Briefmarken-Preisbuch Europa 1908. Leipzig: Selbstverlag 1907. 236 S. m.Abb. $8^{\circ}$.

— Desgl. 1909. Ebda. 1908. $256 \mathrm{~S}$.

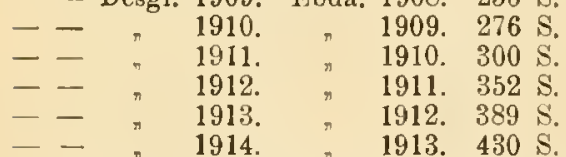

- Die Postfreimarken und die Entwertungen der deutschen Postanstalten in den Schutzgebieten und im Auslande. Mit über 800 Abbildungen. Leipzig: Selbstverlag 1908. III, 127 S. 80
Friedemann, Albert: Preis-Liste $1906 / 07$ der Briefmarkenhandlung A. F., Leipzig, Josephinenstr. 19 (vom 5. Januar 1907 ab: Leipzig, Härtelstr. 23). Leipzig: Albert Friedemann (1906). $40 \mathrm{~S} .8^{\circ}$.

- Preis-Liste 1907/08 der Briefmarkenhandlung A. F., Leipzig, Ilärtelstr. 23, über Echto Briefmarken in Sätzen u. in Paketen. Ebda. (1907). $32 \mathrm{~S} .8^{\circ}$.

- Preis-Liste 1909 der Briemarkenhandlung A. F.... über Echte Briefmarken in Sätzen u. Paketen, Briefmarken-Albums u. SammlerBedarf. Ebda. (1909). 44 S. 8\%.

- 1910... Ebda. (1910). 48 S. 80.

_ - 1911 ... Ebda. (1911). 64 S. m. Abb. $8^{0}$

— - 1912 ... Ebda. (1912). 72 S. m. Abb. $8^{\circ}$

- - 1913 ... Fbda.(1913). 88 S. m. Abb. $8^{\circ}$.

- Preisliste für echte Briefmarken in Sätzen u. Paketen 1914. Leipzig: Albert Friedemann (1914). 72 S. m. Abb. $8^{\circ}$.

- 1916. Ebda. (1916). 76 S.m. Abb. $8^{\circ}$.

- Preisliste 1907/08 der Briefmarkenhandlung A. F. . . über Briefmarken-Albums n. sonstige Sammler Bedarfsartikel. Leipzig: Albert Friedemann (1907). 16 S. m, Abb. $8^{\circ}$.

- Preisliste 1914 über Briefmarken-Albums u. Sammler-Bedarfsartikel. Ebda. (1914). 12 S. m. Abb. $8^{\circ}$,

- 1916 ... Ebda. (1916). 12 S. m. Abb. $8^{0}$.

- Proben und Entwürfe für die Marken der Deutschen Reichspost und der Deutschen Schutzgebiete. Leipzig: Selbstverlag [1909]. 12 Bl. in Mappe. $8^{\circ}$.

[Hiervon erschien eine Sonderausg fiir die Besucher des Sammlertages in Gotha mit Widmung in Gold druck.]

Friederich, Rudolf: Die Postwerthzeichen Spaniens und seiner Colonien, auf Grund bauptsächlich spanischer Quellen bearbeitet. T. 1. Die Postwerthzeichen Spaniens. Mährisch-Ostrau: Verlag des "Philatelistischen Börsencourier (Kittl) 1890. 66 S. m. Abb. $8^{\circ}$. - Zweite Aufl. 2 Teile in $1 \mathrm{Bd}$. Berlin: Dr. Hans Brendicke 1894. $8^{\circ}$.

T. 1. Die Postwerthzeichen Spaniens, 160 S. m. 63 Abb. u. 13 'Taf.

T. 2. Die Postwertzeichen der spanischen Colonien. 176 S. m. 14 Abb.u. 7 Taf.

[Hiervon gibt es eine Aueg. auf Schreibpapier gedr. ע. beide Teile in Leinw. oder Halbfr. geb.l

- $-\mathrm{Y}^{1}$. . s. Berlin (Berliner PhilatelistenKlub). Festschrift z. Feier des zehnjährigen Bestehens des B. Ph.-Kl. Berlin 1898.

Friedl, Rudolf: Illustrierter Spezialkatalog und Preisliste sämtlicher Post- und Telegrafenwertzeichen von Österreich-Ungarn. [Umschlagt.]: Die Postwertzeichen von Österreich-Ungarn. Wien: R. Friedl 1897. $72 \mathrm{~S} .8^{0}$.

- Taschenatlas für Briefmarkensammler. Wien: Rudolf Friedl 1908. $8^{\circ}$. 
Friedl, Signund: Ausstellungs-Katalog res Internationalen l'ost-Werthzeichen-Museums 7u Wien. Wien: S. Friedl 1883. 12 S. $8^{\circ}$. - [Neue Ausg.] Elbda. 8. 80.

- Zur (Yeschichte der Österreichischen Mercure 1850-56. (Sonderabdruck aus der ${ }_{\pi} \mathrm{Welt}$ post“.) Wien: S. Friedl. 8 S. m. Abb. 4".

- llandbuch und Preiskatalog aller Post- und T'elegraphen-Verthzeichen der Erde. Mit Berücksichtigung der hauptsiichlichsten Nuan cen, Fehldrücke, Local-, Trivat- und Specu lationsmarken. Wien: Sigmund Friedl I883-1884. (Aegypten-Italien.) I81 S. 4\%. [Uavolleadet ]

- Nllustrirter liatalog sämmtlicher bis 1876 erschienenen Briefmarken. (Der plilatelistischen Kataloge T. I.) Wien: Selbstverlag 1877. 167 S. So.

- lllustrirter Katalog sämmtlicher bis Anfang 1878 erschienenen Briefumschläge, Streifbänder etc. (Der philatelistischen Kataloge T'. 2.) Wien: Selbstverlag 1878. $62 \mathrm{~s} .8^{\prime \prime}$.

- Illustrirter Katalog sämmtlicher bis Anfang 1878 erschienenen Pestkarten, Anweisungen, Mandate, Nachnahmen. Postfrachtbriefe etc. (Der philatelistischen liataloge T.3.) [Nebst:] Nachtrag zu T. 2. Wien: Selbstrerlag 1878. 53 \$. $8^{\circ}$.

- Illustrirter Nachtrags-Katalog (Der philatellitischen Kataloge T. 4), enthält alle bis 1879 neu erschienenen Postwerthzeichen und vorgelrommenen Richtigstellungen zur Completirung der permanenten 3 Theile illustrirter Kataloge. Wien: Selbstverlag 1879, $50 \mathrm{~S}$. $8^{\circ}$.

- Nachtrag [ ${ }_{\pi}$ lllustr. Katalog ${ }^{*}$. Ebcla. 1880. 24 5. [Einseitig gedr.]

- Nachtrag [ IIlustr. Katalog*]. Ebda. 183I. 15 s. [Einseitig gedr.

- llistrierter Postwertzeichen-liatalog. Enthaltend alle bis I891 erschienenen Postmarken, postalisch gebrauchten Stempelmarken, Briefumschläge, Postkarten, Kartenbriefe, Postanweisungen, Streifbänder, Packetbegleitadressen u. Ritckscheine einschlieflich FehIdrucke, Typen u. Neudrucke unter Beriicksichtigung aller Verschiedenheiten, bezüglich Zihnungen, Wasserzeichen, Flaggenstempel, Formate, Gummierungen etc. Wien: Sigmund Friedl 1892. $544 \mathrm{~S}$. sehmal $8^{\circ}$.

[Siehe auch "Eckelmann, P. \& Co. "u. "Senf, (rebrïder" unter gleichlautendem Titel gedruckt.]

- Manco Liste [zum nIlustrierten Katalog"] Wien: Selbstveri. [1879]. $16 \mathrm{~S} .8^{\circ}$.

- Die Postwerthzeichen von Bayern. (Vortrag, gellalten im Wiener Philatelisten-Club am I. Juli 1850.) Wien: Verlag der "Weltpost" (S. Friedi) 1880.8 S. m. Abb. $8^{\circ}$.

Friell \& Baun. Intern. Postwertzeichen-Aus. stellung IVien 1890. Photografien dor hervorragendsten Objecte der Ausstellung... IVien I890. 2 S. m. I8 Phot. auf líalton.
Friedłänder, l'aul: Bassaher. s. Lietlin. (lierliner Philatelisten-kilub) in: Festschrift z. Feier des 25 jïhr, Bestehens des B. J'h. Kl. lierlin 19I3.

Friedrichsehe Prachtsammlung s. BriefuarlienVersteigerungen Paul liohl. [Katalog Nr.5.]

Fibrer, Der, im Labyrinthe der bisher erschienenen Bricfmarken s. Schubert, $G$.

Galerie Helbing s. Briefmarken-Versteigerungen (Kataloge: Paul Kohl, Chemnitz).

franzsachen. Sellschopp, IVilhelm: Preisliste über Ganzsachen. Hamburg 1904.

Teichmann, Dr.: schutz den Gauzsacien? Dresden [I890].

Thier, Max: Gegen das Ausschneitien von Ganzsachen. Antrag 4 auf dem 11. Meutsch. Philatelisten-Tag zu Frankfurt a. Ml. I890.

(Xarantiestempel, Über, s. Töpfer, J. Ad. (Vortrag, geh. auf dem IV. Deutschen Philate. listentag in Prag I892.)

Gast, Richard: Katalog aller bekannten, seit 1840 bis Fnde 1874 emittirten Briefmarken. $\mathrm{Zu}$ den beigesetzten Preisen zu haben bei $\mathrm{l}$. G. Dresden: Selbstverl. 1874. 34 S. 4".

- Katalog aller bekannten, seit 1840 lis linde 1876 emittirten Briefmarken . . bei li. G. Ebda. $1876.42 \mathrm{~S} .4^{\circ}$.

Katalog altdeutscher Postwerthzeichen. Ebda. (1890). 14 S. $4^{0}$.

[в, a. Dauth, H. J." u. "Freudenstein, S. ${ }_{\eta}$ unter dem gleichnamigen Titel gedruckt.]

Gebraucht $u$. ungebraucht. s. Sellschopp, W. (Vortrag, gehaiten auf dem XIII. Deutschen Philatelisten-Tag. Berlin 190I.)

(ienth, F.: Abstempelung und Entwertung auf Narken Portugals und seiner Kolonien. Vortrag, geh. auf dew Begrühungsabend des 17. Ringtages zu Cassel, I6. Juni 19I1. (Sonderdruck-Beilage zu Kohl's Mitteilungen.) Chemnitz: PaulKohl 1911.24 S. u. 8 Taf.m. 1bb. $8^{\circ}$. - Übersicht über die in den Jahren 1840-191 I ausgegebenen und verwendeten geteilten Marken. Cassel: Selbstverlag 1911. 18 S. $8^{\circ}$.

- Wie es ist, wie es nicht ist und wie es doch sein könnte! Vortrag. s. Deutscher Philatelistentag; in: Stenograph. Bericht des XXV. D. Ph. . . zu Hannover 1913.

feorg, Wilhelm: Katalog über alle seit 1840 bis IS64 erschienenen Briefmarken, welclie zu den beigefügten Verkaufspreisen durch IV. G. in Basel bezogen werden können. Basel und Genf: H. Georg (1864), 99 S. $8^{\circ}$.

Gerdt, A. [d. i. A. Gerhauser]: Herrn Schwofelmeier's zeitgemäfe Betrachtungen itber die Farbenbezeichnungen in der philatelistischen Litteratur. [2. Aufl.] Lcipzig: Gebrüder Senf (I89I). 48.80

Germania-Handbiicher. Bd. I. l'atbtafeln. Für Postwertzeichen Sammler. Hrsg. vou P. Ohit. Düsseldorf 1906.

Bd. 2. Neudrucke. Handbuch aller bekannten Neudrucke staatlicher Postfreimar- 
lien, lianzsachen 4. Hssays... Bearb, von l'. Ohrt.

ๆ. 1. Afghanistan-Hamburg. Düsseldorf 1907

2. Hannover-Marshallinseln. Düsseldorf 1912

3. [lm Erscheinen.]

liplnania-Ring. Mitglieder-Verzeichnis des (iermania-Rings /Verband deutscher Postwertzeichensammler. Vereine, E. V.) neust einem Anhang, enthaltend:

t. Satzungen des Germania-Rings,

2 - des Kauf-u. Tauschverkehrs,

3. der Schadenersatzkasse

4. dler Briefmarkenbörse,

5. Geschäftsordnung der Ringtage,

6 der Sammlertage,

7. $\rightarrow$ der Neubeiten-Kaufstelle,

8. der Prifungsstetle,

$4 . \quad$ der Bücherei.

Stand am 15. Juni 1914.

Leipzig-Breslau: Selbstverl. des GermaniaRings 1914. $124 \mathrm{~s}$. 80 .

- Schwarzer Permanent-Katalog zum Sehutz geren nicht empfehlenswerte Adressen. Mit periodischen Nachträgen hrsg. vom GermaniaRing. Berlin 1902. IT, $72 \mathrm{~S} .8^{\circ}$.

Gesantinteressen der Briefmarkenkunde. schindler, A.: Gründung eines Centralorganes für die Gesamtinteressen der Briefmarkenkunde. Antrag 3 auf d. II. Densteh. Philatelisten-'Tag zu Frankfurt a. M. 1890.

Gibraltar. Breimeier, W.: Die Postwerthzeichen von Gibrattar seit 1839. Leipzig 1892.

Fohl, Paul: Illustr. Katalog der Freimarken von Europa, Abt. 3. "Gibraltar".

Gießmann, W.: Internationales PhilatelistenAdreBbuch mit Angabe der Sammelobjecte, 'Tausch-, Kauf-und Verkaufsbedingungen ete. der Sammler und Händler ... Saalfeld, Leipzig: Ernst Heitmann 1898. X, 212 S. $8^{\circ}$.

[Das Werk erechieu unter gleichem Titel, jedoch ohne Nomenenenung des Verfassers jm Jahre 1902 bei Hermenn Dege in Leipzig.] $\theta$. a. u. d. T. Adressen.

(kirsewall, (. .: Die Schweizer Marken von 1813 bis 1854 . Studie anläblich des 50 jïhr. Iubiläums der ersten schweizerischen Postmarken. (Sonderabdr. aus der Postwerthzeichenkunde, April-Juni 1893.) Minchen: A. Larisch 1893. 20 S. m. Typen-u. TtempelAbb. 80 .

Glasewald, Arthur Erust: Die Abstempelungen der Marken von Baden. Unter Mitwirkung mehrerer Specialisten bearb. von A. E. F. GöBnitz, S.A.: A. F. Glasewald 1898. 96 个. m. 2 Taf., 1 Brief iacsinile $n$. 112 Abb. i. Text. $8^{\circ}$.

Die Abstempelungen der Marken des Thumu. Taxis'schen Postgebietes. Nach mehreren Vorträgen (unter Zugrundelegung der eigenen Sammlung bearb. von A. E. G. Göb nitz, S.-A.: Buchhdlg. Arthur Glasewald 1893. [Tmsehlag] $1894.60 \mathrm{~S}, 10$ Taf. u. 2 liarten. 80 filasewall, Irthur Erust: Baden-StempelVerzeichnis. (Sonderablruck aus Mitteldeutsche Philatelisten-Zeitung 1894.) GöBnitz: Selbstverl.) $4 \mathrm{~S} .8^{0}$.

- Ver Ballast in der Philatelie. Vortrag. s. Deutscher Philatelistentag; in: Stenograph. Bericht ii. d. XIV. Deutschen PhilatelistenTag ... in Wien 1902.

- Permanenter Briefmarken-Katalog. Deutschland. Mihrisch-Ostrau: [K. C. Sauer 1888.] $40 \mathrm{~S} .8^{\circ}$.

- Don Carlos.Marken. Vortrag zum 26. Philatelistentag in Cassel, gehalten rom Bundesprüfer A. F. G. Sonderabdr. aus Philatetisten-Zeitung. " GöBnitz, S.-A.: A. E. Glasewald 1914. 2 S. u. I Taf. m. Abb. $4^{\circ}$.

[Der Vortrag war fir den 26. Philatelietentag uแ. vorbereitet; infolge Kriegeauehriclis fel der Philatelistentag aue, der Vertrag wurde jedech vom Verfosser auderweitig in philatelietiechen Vereivea gehalten und erschien auch im Druck.]

- Die historische Entwicklung der cieutschen Poststempel. Vortrag, gehalten am 25. deutschen Philatelistentag in Hannover. (Sonder. abdr. aus .Philatelisten-Zeitung ${ }^{*}$ 1913.) (GöBnitz, S. A. : Selbstverlag 1913. 4 S.m. Abu. $4^{\circ}$.

- Postalisch verwendete Fälschungen. Vortrag. s. Deutscher Philatelistentag; in: Stenograph. Bericht ii. d. XVII. Deutschen Philatelistentag . . . in Regensburg 1905.

- Kurze fieschichte des Mitteldeutschen Philatelisten - Verbandes nebst Vorgeschichte $\langle 1887-1894\rangle$. Zum 5jähr. Stiftungsfeste zusammengest. von A. E. G. Gähnitz, S.A.: Selbstverl. 1894. 14 S. 80.

- Katalog und Mancoliste såmmtlicher bis Ende September 1887 herausgegebenen deutschtn Privatpost-Marken. [2. Autl.] GöPnitz, S.-A.: Buchhdlg. Arthur Glasewald 1887, 25 5. 8.

_ - 3. Aufl. T. 1: Marken ... his Ende No vember 1887. Ebda. 1887. 104 S. $8^{0}$.

T. 2: Ganzsachen . . . bis Ende 1887. Ebda. 1887. 115 S. $8^{\circ}$.

- 4. Autl. Kiatalog ... bis April 1888. Euda. $116 \mathrm{~S} .8^{0}$.

[Dic 4. Aufl. orechien auch als Supplement zu deu ${ }_{n}$ Neuesten Privatpost-Nachrichten ${ }^{4}, J g .1$. 1888.1

- Lager-Verzeichniss der PostwerthzeiclenHandlung von A. F. $\left(\frac{1}{1}\right.$. . . Weilnnachten 1888. Fößnitz, S.-A.: A. E. Glasewald 1888. 16. so.

- Neue Ausg. Fbda. 1890. 22 S. 80.

- Die Levante und deren Posten: Vortrag für den XXIV. Pliilatelistentag in Marktredwitz, gehalten ron A. F. 1\%. Sonderabdr: aus Philatelisten-Zeitung"1912.] GöBnitz, S. A.: A. E. Glasewald. 4 S. m. 32 Stempel-Abb. im Text. $4^{\circ}$.

- Die Nachportomarken von Griecheuland. (Sonderabdr. a. d. Mittheilungen d. Oesterreichischen Phil.-Clubs.) Wien: W. Jacobi 1890. 3 S. m. Abb. $4^{\circ}$.

- Neudrucke von Helgoland. Mit 35 Neudrucken bektebter Karton. roönitr. S.-A.: Selbstverlag 1910. 
Mlasewald, Inthur lirusl: Die Neuclrucke von Kirelıenstaat. Mit 50 Neutrucken beklebter Karton. GöBnitz: Sellustverlag 1910.

- Neues und Vergessenes iliber Bulgarien und Rumelien. Vortrag, gehalten auf dem X. Deutschen Plilatelistentag in Göbnitz. Fonderabdr. aus Mitteldeutsche PlilatelistenZeitung" 1898.1 GöBnitz, S.-1.: A. K. lilasowald 18.98. 4 S. m. Marken-u. Stempel-Abb. in Text. $4^{\circ}$.

- Iie Post im liriege. Beiträge zur Geschichte der feldpost. Gö́nitz, S.-A.: A. E. Glasewald 1914. 240 S. m. Abb. 80 .

lisschien ursprünglich getrent in 2 Teilen T. 1. S. $1-100$ (1912).

T. 2. S. $101-240(1913 / 14)$.]

- Die Postmarken von Griechenland. Philitelistische Studie s. Isakovies, A. v.: Internationales Tausch., AdreB. 10. Hüilfsbueh tül den Tauscliverkehr, T. 2.

- - s. a. u. Erdmann, A. (pseud.)

- Die Postwerthzeichen von Griechenlaurl. Nach den neuesten Forschungen bearb. von A. E. G. GöBnitz, S.-A.: Selbstverl, 1896. 64 s. $8^{0}$.

- Preisbuch "Europa", zugleich Manco-Liste zum Universal-Briefmarken-Album (Verlag von Hupfeld u. Gröber). Ausgegeben Lezbr. 1890 von der Briefmarkenhandlg. A. E. Cilasewald. GöBnitz, S.-A.: A. F. Glasewald 1890. 50 S. $8^{\circ}$.

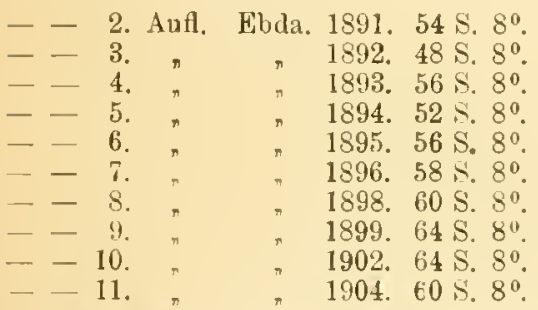

Restbestiinde oder Neudrucke altdeutscher Marken nebst deren Verbrauchszeit. [Zum 25 jïhr. Jubiläum des Händler-Vereins Berlin 1911.] (Extra-Beil. zur Philatelisten-Zeitung.) (rößnitz, S.-A,: A. F. (rlasewald 0. J. [Einblattdruck] $4^{0}$.

- Restbestände u. deren Schicksale. Vortrag. s. Jeutscher Philatelistentag; in: Vorträge, gehalten auf dem XX. Deutschen Philatelisten-Tage zu rößnnitz 1908.

- Verzeichnis der in Gruppen auftretenden Nummern-, Orts-etc. Stempel der deutschen Finzelstaaten u. Kolonien i. A. des Verbandes der Sammler von Postwertzeichen nach Abstempelungen bearb. von A. H. C. Cöbnit\%, S.-A.: Verband der AbstempelungsSammler 1905. 320 S. $8^{0}$.

[Verüftentlicht in 8 Einzelliefgu.]

- Nachtr. Ebda. 1906. 109 \&. 8\%.

[Teilg nur einseitig bedr.]

- Wie können die Sammler gründlicher als bisluer vor Betrüigern ฉ. Fiilschern gewarnt u. geschützt werden? s. Deutscher Plilate-
Iistentag: Antrag 2. in: Bericlit über den I1. Leutschen Plilatelisten-L'ag zu Frankfurt a. N. 1890.

lilasewald, Mriur Erust: Wie velbessern wir unsere Tauschverbindungen? Vortrag. s. Deutscher Plilatelistentag; in: Stenograph. Bericht i. d. Verhandlungen des TII D. I'h. zu Mannheim 1895.

- Die Zukunft unserer Tauschverbindungen. Vortrag. 3. Deutscher Philatelisten-Tag; in : Stenogr. Protokoll ii. d. Sitzung des XII. Deutsch. Philatelisten-Tages ... in Frankfurt a. M. 1900.

filasewa1, A. E., O. Satller a Fr. Iragner: IIandbuch der deutschen I'rivat-Pestzeichen. 2 Bde. 8\%. Gölnitz, S.-A.: Buchhdlg. Arthur tilasewald.

Bd. 1. (H. 1-11.) Altona-líreiz. 1889. 324 s.

Bd. 2. (II. 12.) Hamburg-Haunover. 1890. 40 s. [Unvollendet.]

Glasewald, Erust: Biograph. Shizze von Chri stian WeiB in Philatelistischer Börsen-Courier, VI. Jahrg. 1891. Nr. 13.

Gobbert, Fr.: Verzeichnis sämmtlicher amt. lich ausgegebenen Briefmarken der Staaten des deutschen Bundes bis zur Errichtung des deutschen Reiches. Breslau: Fustav Liurmeister 1891. $29 \mathrm{~S} .80$.

(iojpfert, R : Staatspost und Privatpost. Versuch einer Erläuterung der Stellung der Staatsverkehrsanstalten im deutsehen Reiche gegenibber Privatverkehrs-Anstalten. Vom fachnänn. Standpunkte unter Berücksiclit. der geschichtl. Entwiekelung der Post u. der Postgesetzgebung bearb. von R. (., Kaiserl. Postdirektor. Dresden: Wilhelm Baensch 1887. 43 S. $8^{\circ}$.

Goslar. Denkseluift zur Einweihnng des neuen Reichs - Postgebändes zu Goslar an 28. Mai 1894. Braunschweig: Buchdruckerei Albert Limbach [1894]. $8^{\circ}$.

fiobnitz, S.-A. (Deutscher Philatelisten-Verband.) Bibliotheks-Verzeichnis des Deutsclien l'hilatelisten-Verbandes. (Stand am 1. Juli 1897.) GöBnitz, S.-A., 1897. $8 \mathrm{~S} .8^{\circ}$.

- Verbands-Nachrichten des Leutschen Pliil atelisten-Verbandes. s. Abt. "Zeitselıriften".

- (Mitteldeutscher Philatelisten-Verband.) Mitglieder-Verzeichniss des MI. Ph.-V. Stand vorn 15. Februal 1891. GöBnitz, S.-A. 1891. 8 S. $8^{\circ}$.

- Verbands-Naehrichten des M.Ph.-V. \&. Abt. "Zeitschriften $"$

liottschalk, Albert: Die Aufdrucke auf den Postmarken. (Sonderabdruck aus "Der Philatelist* 1895, Nr. 1-6.) Berlin: Sielbstverlag. 20 s. $8 \%$.

- Die deutsche philatelistisclie Literatur 1894 Jis 97. s. Berlin (Berliner Philatelisten-Klub) in: Festschrift z. Feier des zehnjailurigen liestehens des B. Pli.Kil. Berlin 1898. 
Grauberg, Jh.: Die Ganzsachen von Finnland. lierlin: Plilipp líosack 1903. 24 S. m. 63 Abb. 80

(Yraz (lnneıösterreichischer Philatelisten-Club). Bestimmnngen für den Tausch- und haufverkehr des 1. Ph.-CI. Graz: [Selbstver]. 1888.] $48^{\circ}$.

- (Verein der Briefmarkensammler.) Fest schrift anläBlich des 50 jährigen Jubiläums der ersten Oesterreichischen Briefmarke. ( $/ \mathrm{mm}$ 14. Stiftungsfest des Vereins am 21. Mär\% 1900.) (Araz: Terein der Briefmarken-Sammler $1900.12 \mathrm{~S} .4^{\circ}$.

[Autographiert.]

Griebert, llugo: Montenegro. Spezial.Album [und Katalog] fiir die Briefmarken Monte negros. Iondon: 11. Griebert (1913). $76 \mathrm{Bl}$. z. T. m. Vordruck. Quer $4^{0}$ (Quarteblong).

(iriechenland, Glasewald, A. E.: I)ie Nachportomarken von Griechenland. Wien 1890.

- Die Postmartien von Griechenlands. 1sakovics, A. v.: Intern. J'ansch., AdreB- u. Hiilfsbuch $\mathrm{f}$. den Tauschverkelır 2.

- - s. a. Erdmann, Arthur.

- - Die Postwerthreichen von Griechenland. Gölinitz 1896.

- Kohl, Paul: 1llustr. Katalog der Freimarken von Europa. Abt. 8. "Griechenland."

Grilstad, 0laf: Briefmarken-Preishiste. Drontheim (Norwegen). 1. August 1892. 84 S. $8^{\circ}$ $\left(16^{\circ}\right)$.

_- [Neue Ausg.] Ebda. 1. Novbr. 1893. 36 S. 40

- Hngros-Preisliste. Drontleim (Norwegen). 1. Dezbr. 1891. 14 S. $8^{\circ}$.

- - [Neue Ausg.] Ebua. Septbr. 1892. 12 S. $8^{\prime \prime}$.

_- Neue Ausg.] Ebda. 1. Mai 1896. $10 \mathrm{~S}$. So.

- - [Nene Ausg.] Hbda. 1. Novbr. 1896. 8\$. $8^{0}$.

- Preisliste äber Skandinavische Briefmarken. Irontheim (Norwegen). Septbr. 1898. $12 s 8^{\circ}$.

- -.. [Neue Ausg. Ebda. Juni 1899. 10 \$. 80. Preisliste iiber Skandinavisclue Briefmarken, Couverts, Postkarten etc zn beziehen von O. G. Diontheim, Norwegen, Juli 1889. $14 \mathrm{~S}$. 8".

- - [Noue Ausg.] Ebda. August 1890. $16 \mathrm{~S}$. - - [Neue Ausg.] Ebda. 1. Iuni 1891. $16 \mathrm{~S}$.

Grönlaul, Eine Schwindelemission; in: Moschkau, A., Zur Geschiclıte der Philatelie.

Goßbritanuien. Castle, M1. P.: Die selteneren Marken von GroBbritannien. Jierlin 1898.

- Ewen, H. L'Estrange: Katalog u. Preisliste der Brief- u. Telegraphenmarken von Großbritannien, übers. v. F. D. Frank. Frankfurt a. M. 1895 .

[8. a. u. Frank, E. 1).]
(Arobbritannien. Kohl, Paul: Illustr. liatalog der lireimarken von luropa. Abt. 3. "Lirol.. britannien."

Westoby, W. A.S. Bleuté par la gomme nebst der deutschen Übersetzung! Vom Trummi geblitut. Berlin 1898.

(irosse, Oskar: Die Beseitigung des Thurn und Taxisschen Postweseus in Deutsclland durch Heinrich Stephan. Minden: J. C. C. Bruns" Verlag 1898. 131 S. 80.

- Dic deutsche Felipost. 31.-35. T'aus. Berlin, Verlag Kameradschaft (1915). 64 S. $8^{\circ}$. (Unterm Eisernen Kreuz 1914)15. Kriegsechriften deg Kaiser-Tilhelm-Dank. Verein der Soldatenfreunde, H. 24.)

Die deutsclie lieldpost im Weltkriege. (Tortrag m. Lichtbildern gelialten Ende $1915 \mathrm{im}$ Wissenscliaftl. Theater der Berliner Urania.) Sonderabdr. a. "Westermanns Monatsheften". Braunschweig, Berlin, Hamburg: George Vestermann (1916). 18 S. mit $14 \mathrm{Abb}$. im T'ext. $4^{\circ}$.

(irosse, IR.: Das Postwesen in der Kurpfalz im 17. ๒. 18. Jahrhundert. Tübingen: J. C. B. Mohr 1902. 67 S. $8^{\circ}$.

Grossnanu, F. W.: Katalog aller seit 1840 bis 1874 ausgegebenen Briefmarken. Zu den beigesetzten Preisen zu haben bei F. W. G. Dresden: Selbstverl. 1874. 50 S. $8^{\circ}$.

- - 2. Aufl. Ebda. 1876. 60 S. $8^{\circ}$. [3. $\rightarrow$ 1878. $51 \mathrm{~S} .8^{\circ}$. 4. $\pi, 1880.47$ S. $8^{\circ}$. 15., 1880.47 S. $8^{\circ}$. $-[6 . ;], 1880.47$ \&. $8^{\circ}$.

lirotrinn, Ludwig: Preisliste Nr. 1 der Briefmarken-Handlung I. G. Hamburg 1890. 22 S. $8^{\circ}$.

- Preisliste Nr. 2 .. Hamburg 1892. 75 S. $8^{\circ}$.

(iruulziige der Briefmarkenkunde u. des Briefmarkensammelns. s. Suppantschitsch, Victor.

Guumirung. Westoby, W. A. S.: Blenté par la gomme [nebst deutscher Übersetzung]: Vom Gummi gebläut. Berlin 1898.

(tiindel, Carl ron: Biographie; in: Teran, O.: TVegweiser für Verkehrswertzeichen-Sammler.

- Die Chalmers-Hill'sche Streitfrage. Ebda.

- Die Postkarte, ibro Erfindnug u. ihre Verbreitung; in: Senf, Gobr.: Taschenbuch für Briefmarken-Sammler 1889.

Gutnienseh, J.: Peru [Katalog]. Verfasst mit

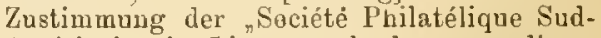
Américaine in Lima" nacb dem von dieser Gesellschaft herausgegebenen Kataloge. Frankfurt a. M. Terein für Briefmarkenkunde 1887. $12 \mathrm{Bl}$, einseitjg bedruckt in grauem Unschl. m. ïber 260 Feldern f. die Postmarken Perus vom 1.12.1857 bis 15.9.1886 zum Einfügen in das Scliaubek-, sowie jedes andere Quart-Album. 4\%. (Druek der Benedictiner Bucladruckerej in Briinn in AlbumForm.)

- Permanenter Iriefmarken-liatalog. Portugal und seine Colonien. Mälrisch-Ostrau [K. Cl. Sauer 18ss]. 36 s. 8*. 
IIack, IIrmaun: Haack's PhilatelistenAdressbuch 1910. VerfaBt ron Herm. Haack, Crefeld. 2. verm. Aufl. Crefeld a. Ris.: 11. llaack [1909]. 122 S. $8^{\circ}\left(16^{\circ}\right)$.

(1)ie 1. Aufl. erschien 1908 eiuseitig bedr.]

- - 1913. 3. Aufl. Ebda. (1913). 212 S. 8" IIaack's Philateliston-Kalender 1910/11. Ueberreicht von Herm. Haack, Verlag des "Philatelisten-ArireBbuch", Zentralo für pbil. atelistische Literatur u. Bedarfsartikpl: Briefmarken-Import. Crefeld 〈Rhli.〉, Noristraße 193 (1910). $34 \mathrm{~S} .8^{\circ}\left(16^{\circ}\right)$.

Preisbuch 1912/13 iiber philatel Bedarfsartikel. Philatel. Literatur. Crefeld, Noristr. 139: Selbstveri. 1912. 12 S. $8^{\circ}$.

- - 1913. Ebła. 1913. 12 S. $8^{0}$.

Ilaas, Theodor: Biographie in: Jeran, O.: Wegweiser für Terkehrswertzeicheu-Sammler; - auch in: Isakovics, A. v. Internationales Tausch", Adreb- u. Hiilfsbuch f. d. Taus:hverkehr, T. 2.

- Correferat, betreffend Reform des Vordruckalbums und Agitation gegen das Aufdruckunwesen. Wien 1S85. $4 \mathrm{~S}^{\circ}$. [Autographiert.]

- Uber die Febldrucke mit verkehrtem Mittelstück. Vortrag. s. Deutscher Philatelistentag; in: Vorträge, gehalten auf dem XX. Deutschen Pbilatelisten-T'age zn Gößnitz 1908.

- Die Geschichte unserer großen Rarititen. Vortrag. s. Deutscher Philatelistentag; in: Stenograph. Bericht ü. d. XVI. Deutschen Philatelisten-Tag ... in Leipzig (u. Halle a ২.) 1904.

- Handbuch sämtlicher Postkarten, Kartenbriefe, Postanweisungen u. Paket-liegleitadressen. (Dr. Moschkau: Handbuch für Postwertzeichen-Sammler, VII. Aufl., 11. Bil.) Leipzig: Gebrüder Senf. (1891.) VI, 330 S. $8^{\circ}$.

- Lebrbuch der Briefmarkenkunde. Ein Hülfs. und Nachschlagebuch. Nit 5 Portr., über $1300 \mathrm{Abb}$. u. mehreren ganzseitigen Taf. sowie einer farb. Darstellung verschiedener Druckarten.? Leipzig: Gebr. Senf (1905). X. $577 \mathrm{~S}$. nebst $1 \mathrm{Bl}$. Berichtigungen. $8^{\circ}$.

Errchien erstmalig bogeuweise alo Beilage zun Illustr. Briefmarken-Journal. JR. 1903-1905, вp̈̈terhin erschien dann ejne Ausgabe auf besserem Papier gedruckt.]

- Katalog zu der vom Bayerischen Philatelisten-Verein München in der ersten OktoberHälfte 1884 veranstalteten Postwerthzeichenausstellung. Zsgest. ron Theodor Haas, Vor* sitzender des Ausstellungs-Comités. München: Selbstverl. des Bayeriscben Philatelisten-Vereins 1884. 11 S. u. 2 Facsimiles der "Mulready"-Briefumschläge. $8^{\circ}$.

- Leitfaden der Briefmarkenkunde. 4. Autl. Leipzig: Gebr. Senf 0. J. (1914). S".

- Über die augeblichen bayrischen Neudrucke. Vortrag. s. Deutscher Philatelistentag; in: Stenograpb. Bericht über den 5. I. Ph, in Berlin 1893.
Has, Theodor: Die ailtesten Postwerthzeichen von 1653 in: Isakovies, A. v. : Intern. T'ausch-, Adref-u. Hïlfsbuch f. d. Tauschverkehr. T. 2.

- Die Postwertzeichen des Suaheli-Sultanates. s. Berlin (Berliner Pluilatelisten-Klub) in: Festsclurift z. Feier des zehnjähr. Bestehens des B. Ph.*Kl. Berlin 1898.

- Tortrag über das Aufdrucksameln, geh. im Bayerischen Philatelisten. Terein am 27 . Februar 1885. Nünchen: Figentum des Bayerischen Philatelisten-I rreins $1885.4 \mathrm{~S} .8^{\circ}$.

- s. a. Moschkau, O. C. A.: Handbuch fïr Postwertlzeichen-Samwler. 7. Auf. lid. 2.

Haass, F.: Entwickelung der Posten vom Alter. tum bis zur Neuzeit. Stuttgart: Metzler's Terlag 1891. $24 \mathrm{~S} .8^{\circ}$.

- 2. Aufl. Berlin: Rosenbaum \& Hart 1895. 26 s. 50.

- Der Meltpostverein. 2. Aufl. Ludwigsburg 1893. $31 \mathrm{~S} .8^{\circ}$

[Auch in Komm, bei Roserbaum \& Hart in Berlin.]

Hagen, Rudolf: Gelegenheitsofferte $\mathrm{Nr}$. 34 a. Leipzig 1904. 43 S. $8^{0}\left(16^{\circ}\right)$.

- $\mathrm{Nr}$. 35. 1904-1905. Leipzig 1904. $199 \mathrm{~S}$. $8^{\circ}\left(16^{\circ}\right)$.

llalbrock. E.: 150 Adressen von anerkannt guten Händlern. Bielefe:d 1872.

IIamburg. Hein, E: Die Postwerthzeichen von Hamburg. IVien 1880.

- Lindenberg, Carl: Die Briefumschläge von Hamburg u Blemen. Berlin 1894.

- - Hamburg; im "Großen Handbuch der Philatelie" Bd. 2. S. 181-188. Leipzig 1889.

- Lossau, Julius: Hamburg, (seine Post u. seine Postwertzeichen). Vortrag, geh. auf dem 9. Deutsehen Philatelistentag in Hamburg 1897.

- Obrt, Paul: Notizen über Hamburger Postfreimarken. [Charlottenburg 1900.]

- Die Hamburg'scben Steindruckmarkeu $11 / 4$ u. $2 \frac{1}{2}$ Schilling der ungezähnten Ausgabe 1864. Berlin 1898.

- Fommel, Otto \& Hugo Krötzsch: Hamburg (Krötzsch, Handb., Bd. 7). Leipzig 1893 bis 1896.

- Ruben, M. E.: Vollständige Preisliste von . Hamburg . . Kopenhngen 1889,90.

- Vicenz, Ernst: Gibt es amtliche Neudrucke u. Probedrucke von Hamburger Steindruckmarken? Dresden 1907.

- (Hamburger Briefmarken-Börse.) Gesetze der Hamburger Briefmarken Börse. Revidiert 1. Novbr. 1871. Hrsg. vom Vorstande. Hamburg: Selbstverl. 1871. 4 S. $8^{\circ}$.

Hamilton-Vertrag. Weegmann, F.: Stellung nahme gegen den Hamilton'schen Virtrag mit den Centralamerikanischen Republiken. 8. Antrag 1 auf II. Deutsch. PhilatelistenTag zu Frankfurt a. MI. 1890. 
Ilancian, Louis: Die Diligencias von Uruguay. s. Berlin (Berliner Pbilatelisten-Klub) in: Festschrift z. Feier des 25 jähr. Bestehens des B. Ph.-Kl, Berlin 1913.

- Die Postwertzeichen des Grobfürstentums Finnland. Berlin: Philipp Kosack 1909. 59 S. m. Abb. $4^{0}$.

Handbuelı fïr Briefmarkensammles. Anweisung zur zweckmässigsten Einlichtung der Brietmarkensammlungen, nebst vollstäudigej" Ubersicbt und Beschreibung aller bis jetat ausgegebenen Briefmarken. Leipzig: Dürrsche Buchlandlung 1863. 56 S. 8\%.

- Zweite umgear'o. u. vermehrte Aufl. Ebda 1863. 64 S. 80.

Ilandbuch, (iroßes, der Pluilatelie. Verzeichnis u. Beschreibung aller staatlichen u. privaten Post-u. Telegraphen-TVertzeichen sowie der bekanntgewordenen Essais. Mlit vielen erlïuternden Notizen und Bekanntgabe aller Neudiucke unter Mitwirkung bedentender l'hilalelisten. Begonnen von Otto Teltz und fortgesetat von C. Lindenberg, Frhr. von Vittinghoff-Schell, I. H. Anheisser, Dr. F. Kalckhoff und Ernst Heitmann. Leipzig: Ernst Heitmann. 1887-1896. 80.

T. I. Die staatlichen Postwertzeichen.

Bd. 1. Ägypten-Franz.-Guiana. VI, 704 s. 2. Gabun-Mozambique. 1V, $1063 \mathrm{~S}$. 3. Nabha-Neu-Seeland. $132 \mathrm{~S}$.

[Das Werk erschien in 37 Lieferungen und blieb uavollender. s, a. u. Teltz, O.: Großes Handbuch der Philatelie. Uraprüngliche Lieferungaausgabe.]

Iland- I. Lehrbuicher der Philatelie. s. Anheisser, J. H.; Beck, Carl; Berger-Levrault, Oscar; Brendicke, Hans; Haas, Theodor ; Handbuch f.Briefmarkensammler; Heitmann, Ernst; Johannes, Carl; Joessel, Alfons; Kalckhoff, Franz; Kausch, Oskar; Kloss, Paul; Kobl, Paul; Krause, Richard; Krootzsch, Hugo; Lietzow, Paul; Lindenberg, Carl; Meyer, Ferd.; Moschkau, Alfred; Schidlof, Bertbold; Scholtze, Carl Johannes; Suppantscbitsch, Victor; Teltz, O.; v. Vittingboff-Schell; Wenzel, Ernst; Withalm, Hanns.

Hannover. Hoffmann, Emil: Hannover-Durcl. stich. Berlin 1913.

Tíaiser, Wilhelm: Die Postscheine des König. reichs Hannover. o. O. 1. J.

- Kirützsch, Hugo: Hannover. (Krölzsch, Handl). Bd. 8.) Leipzig 1893-1896.

- Lindenberg, Carl: Die Briefumscluäge von Hannover. Berlin 1895.

- Hannover; im GroBen Handbuch der Philatelie" Bd. 2. S. 189-206. Leipzig 1889.

Hanson, 11. G. 5. Frankfurt a. 11. (Verein fül Briefmatkenkunde). Der Durchstich einer Braunscliweiger Marke.

IIartung, Н.: Die Wechselstempelmarke, ihre Verwendung und ibre Fotwertung. (Gratisleil. z. "Illustr. Anzeiger f. Contor u. Bureau, Jg. 1878, Nr. 6.) Neuscbönefeld: Commissionsrath Henze 1878. 16 S. $8^{\circ}$.
Hawai. Kohl, Paul: Jllustr. Freimarken-Kalalog "Englische Kolonien". Abt. 3. Hawai ...

Hayn, Erust: Engros-Preisliste der Briefmarkenbandlung von Fi. II. . . Berlin [1887]. 16. S. $8^{\circ}$.

- Katalog für Briefmarken v. ausgeschnittene Postkarten, Couverts u. Streifbänder der Briefwarkenbandlung en gros $\mathbf{u}$. en detail von $\mathrm{F}$. H. Berlin [1887!] 64 S. $8^{\circ}$.

- Preisliste (Nr.4) der Briefmarkenhandlung von E. H. . . Berlin [1887]. 15 S. 8\%.

Heidelberg. Wagner, F.: Die Privatpost-Wertzeichen ron Heidelberg. Berlin-Friedenau 1912.

lleim, Eduarel: Die Postwertbzeichen von Hanburg. (Vortrag, gehalten in Wiener Phil. Club am 22. Oktbr. 1880.) Wien: Sigmund Friedl 1880, 2 S. 10. Abb. 8".

Ileinlolı, ..: L'riefmarken-Sammler ט. Briefmarkenhandlungen aller Welttheile und Länler. Erstes philatelistisches Verzeichnis iiber 2100 Adressen; gesammelt und alphabetisch zusammengestellt. München: Selbstverlag 1831. $44 \mathrm{~S} .4^{0}\left(\mathrm{gr} .8^{0}\right)$.

Heitmann, Erust: Heitmann's illustriertes Handbuch für Briefmarkensammler. Prois-u. Mancoliste zu Schwaneberger's Zukunfts-Album sowie jedem anderen Briefmarken.Album. Hrsg. von Paul Lietzow . . 1894-95. 2 Bde. in $1 \mathrm{Bd}$. geb. $8^{\circ}$. Leipzig: Ernst Heitmann 1894.

Bd. 1. XV1, 434 S. u. Preisänderungen. 2. $178 \mathrm{~S}$.

- Neue Ausgabe. 1895-96. 2 Bde. in 1 Bd. geb. 80. Fibda. 1895.

Bid. 1. XVI, $434 \mathrm{~S}$.

$$
\text { ข. } 178 \mathrm{~S} \text {. }
$$

- Das Haus Heitmann; in: Jeran, O.: Wegweiser f. Verkehrswertzeichen-Sammler (Philatelisten Fibe].)

- Manko-Liste zu Schwaneberger's Briefmarkensammelbuch sowie zu jedem anderen Briefmarken - Album [Umschlagt.]: Heitmann's Briefmarken-Katalog und Nanko-Liste zu Schwaneberger's Zukunfts-Album, Leipzig: Ernst Heitmann 1885. V, 227 S. $8^{\circ}$.

- Rundschreiben an die P. T. Abonnenten des Grofien Handhuches der Plilatelie". Fbda. 1893. 12 S. $8^{\circ}$.

s. a. Teltz, O.: GroBes Handbuch der Philatelie. [Lfg. 28-37] 1887[-97.]

- Wandkalender für 1890. (Beilage zur . Jllustrierten Briefmarken-Zeitung *.) Ieipzig : Heitmann $1890.4^{\circ}$.

Hekseh, Iudwig: Die Postwerthzeiclien des Grossherzogthums Finnland. Referirt ron Zahnarzt L. H. in Wien in der Sitzung des "Viener Philatelisten-CJub" am 16. Dezbr. 1880. Wien, Siegmund Friedl [1881]. $12 \mathrm{~S}$. m. Abb. $8^{\circ}$. 
Helgolaur. Filasewald, A. E.: 'Tafel mit den verschiedenen Neudrucken von Helgoland. GöBnitz 1910.

- Kalckhoff, Franz: Helgoland; im nGroBen Handbuch der Philatelie ${ }^{*}$ Bd. 2. S. 207-217. l.eipzig 1889.

- Kohl, Paul: Illustr. Katalog der Freimarken von Europa. Aht. 3, ${ }_{n}$ Helgoland .

- Lindenberg, Carl: Die Postwerthzeichen llelgolands. Vortrag geh. auf dem 6. Deut. schen Philatelistentag in Kiel 1894.

- Pilger, Robert: Helgoland und seine Post. Cuxhaven 1875 .

- Pirl, Paul: Der 3 u. 20 Pf.-Wert von Helgoland im Original u. Neudruck. Berlin 1913. Post. Die Post und Telegraphie auf Helgoland. [Heligoland] 1895.

- Rosenberg, A.: Die Postwertzeichen Helgolands. Vortrag geh. auf dem VII. Deutsclien Philatelisten-Tag zu Manubeim 1895.

- Die Postwertzeichen Helgolands. Bremen 1892.

Wiilbern, Arthur: Originale u. Neudrucke von Helgoland. Hamburg (1906).

Helsingfors. (Helsingfors H'rimärkssamlare Förening) Katalog iiber die Freimarken des (irossfürstentrms Finland. 2. verb. u. verm. Aufl. Helsingfors: Selbstverlag 1904. 18.'. u. 3 T'af. $8^{\circ}$.

- Die Freimarkien des Grossfürstentums Finland [Umschlagt.]: Katalog iiber die Freimarken des Fürstentums Finland, 3. verm. u. verb. Aufl. Ausg. 1908. Kbda. 1908. 333 ś. $8^{\circ}$. [S. 5-33 nnr einseitig bedr.]

Herrmann, Adolf: Druckdaten der Postliarten von Wiirttemberg. s. Berlin. (Berliner Philatelisten-Klub.) Festschrift z. Feier des 25 jähr. Bestehens des B. Ph.-Kl. Berlin 1913.

Herrmann, Emanuel: Biographie; in Jeran, O.: Wegweiser für VerkebrswertzeichenSammler.

- Die Correspondenzkarte. (Drittes Bild der Miniaturbilder aus dem Gebiete der Wirtschaft S. 71-133.) Halle: Louis Nebert 1876. $8^{\circ}$.

Herrmann, Fr.: Die Marken und Ganzsachen altdentscher Staaten, nebst deren Preisen. Leipzig: H. Veit 1891. [Zwei Auflagen.] $8^{\circ}$.

Herrmann, Waldemar s. Moschkau, A.: Handbncl für Postmarken Sammler. 5. Autl. Leipzig 1884 nebst Nachtr. 1885 n. 1886.

Herzog, II. s. Neyer, A.: Die dentsche Post im Veltpostverein.

Itildburglıusen. Human, A.: Geschichte des Postwesens in Hildburghausen. Hildburghausen 1892.

Hildebrandt, Eduard: Die hauptsächlichsten Mängol im Reichspost-u. T'elegraphenwępn. Berlin: Alexander Duncker 1905. $27 \mathrm{~S}$. 8". llildebrandt, Eduard: Jas Postwesen. Kur Reorganisation von Verwaltung u. Betriel). Berlin: E. Nagel 1904. 39 S. $8^{\circ}$.

Ilill, Rowland: Biographie; in: Jeran, 0. : Wegweiser für Verkehrswertzeichen-Sammler. - auch in: Moschkau, A : Beitritge zur Geschichte der Philatelie.

llirsehheydt, A. Y.: Ein neuer Schluss zum Artikel "Wenden". (Sonderabdr. aus der Berliner Briefmarken-Zeitung ${ }^{\star}$ vom 15. Mäı\% 1. 15. Mai 1908.) Berlin: Philipp Kosack 1908. 4 S. $2^{\circ}$. [Vorgang s. Svenson, W.: 'I'ypen u. Abarten der Wenden'sehen Originalmarken.]

Hirt, A.: Preisverzeichnis aller his $1886 \mathrm{er}-$ schienenen Postmarken. Wien: Selustverlag 1886.

Hobrecker, Karl: Die ersten deutschen Luft postmarken. (Sonderdr. a. Berliner Brief marken-Zeitung 1912.) Berlin: Philipp Kosack $1912.7 \mathrm{~S} .8^{\circ}$.

llolfmann, Adolf: Der Illustrierte Briefmarkenfreund. Mannheim [1863]. Hinblattdruck. $4^{*}$. [Nur als Prospekt erschienen.]

lloffmann, Emil : Hannover-Durchstich s. Berlin. (Berliner Philatelisten-Klub.) lestschrift z. Feier des 25 jähr. Bestehens des li. Ph.Kl. Berlin 1913.

II ffrmann, F. A.: lrankreich u. Colonien. (Preisliste.) Paris: Selbstverlag 1897.60 S. 8".

Holkar s. Indore.

Hollands. Niederlande.

Honduras. Hoffmann, E.: Honduras im "GroBen Handbuch der Philatelie" Bd. 2. S. 22l-226. Leipzig 1889.

Hongkong. Hoffmann, E.: Hongkong im „iroBen Handbucli der Philatelie Bd. 2. S. 227-235. Leipzig 1889.

llosp, E.: Preisliste über bayeriscle Marken von der Markenhandlung F. H. München [1884]. 4 S. $8^{\circ}$.

IIruby, Robert: Catalog aller'T'ürkischen, Ostrumelischen, Bulgarischen und Russischen Levantepostmarken, Carten $u$. Couverts mit billigsten Preisen. [Nebst]: Berichtigung. Constantinopel: Selbstverl. 1884. 6 u. 2 S. 8*. Katalog über Orientalische Postwerthzeichen. 'Ksgest. nach Meyer's u. Moschkau's V. Handb. f. Postmarkensammler u. mit billigaten Preisen versehen. Per Śtïck, Satz 100, 500, 1000 u. 2000 Stück. Constantinopel: Selbstverlag 1887. $16 \mathrm{~S} .8^{\circ}$.

- Neue Ausg.| 1892-18\$3. Ebda. 1892. $18 \mathrm{~S} .8^{\circ}$.

[8. a. Antoniades J. T. unter gloichlsutendem Titel gedr ]

_ - Neue Ausg.] 1894-1895. Fbda. Dezhr. 1894. $30 \mathrm{~S} .8^{\circ}$.

Hibel, P.: Deutsches Post- u. 'Telegraphenwesen unter hesonderer Beruicksichtigung der gesamten Literatur. Mïnchen: C. Andelfinger \& Co. 1912. XI, 98 S. $8^{\circ}$. 
II ugelmaun, Carl: Die Concurrenz der Werthzeichen in der Oesterreichischen Briefpost. (Separat-Abdruck aus der "Statistischen Monatschrift".) Wien: Alfred "Hölder 1889. $15 \mathrm{~S} .8^{\circ}$.

lluman, A.: Geschichte des Postwesens in Hildburghausen. Hildburghausen: Radow \& Solnn 1892. 52 S. u. 1 Bildn. S*

IIunimel, Sigmund: Katalog italienischer Militärmarken. München, Dreimüblenstr. 84 : Selbstverlag 1915. 8\%.

Hyllested, Carl: Preise der Briefmarkenliandlung C. H. Copenhagen 18S4. 12 S. $8^{\circ}$.

Iasp, Carl: Postbuch für den Feld- u. KriegsPost- und Telegrammverkehr. Ein Ratgeber in allen Kriegs-Post- $u$. 'Telegraphenangelegenheiten. Bearbeitet von C. J. Berlin: Carl Heymanns Terlag 1915. V1ll, 45 S. $8^{\circ}$.

Jacobs, Carl: Einkaufs-Preisliste der Briefmarken-Handlung C. I [Zweite Aufl.] Goslal a. Harz 1880 . 15 S. $8^{0}$.

- Preisliste Nr. 12. Ebda. 1875. 59 S. 8n.

- Nr. 15. Ebda. 1884. 16 \$. $8^{\circ}$.

- - Nr. 16. Ebda. 1885. 31 S. $8^{\circ}$.

Jacobsen, Nils: Die Zeitbücher der Weltpost. 1. Buch: Die Post der Urzeit oder die Nachrichtenvermittelung vor der Sündfluth. Aus dem Schwedischen übers. Leipzig: Karl Fr. Pfau 1892. 97 S. $8^{\circ}$.

Jahrbuch, Internationales, des Internationalen Sammler-Vereins Hamburg. Ausg. 1911. Hamburg: Verlag des lnternationalen Sammler-Vereins 1911. $8^{\circ}$

Inhalt: Kalender, Reigeausweis, Paetgehührentafel usw.

Jahrbuelı der Philatelies. Wittrisch, Max.

Jamaica. Hoffmann, E.: Jamaica im "GroBen Handbuch der Philatelie" Bd. 2. S. $277-285$. Leipzig $18 \triangleleft 9$.

Jammu s. Kaschmir.

Japan. Beförderung of fcieller Correspondenzen in Japan. in: lsakovics, A. v.: Intern. Tauscl. . Adress- u. Hülfsbuch f. d. Tauschverkehr. T. 2

- (Fälschungen) in Moschkau, A., Zur Geschichte der Philatelie.

- Heitmann, Ernst: Japan; im GroBen Handbuch der Philatelie" Bd. 2. S. 289-320. Leipzig 1889.

Jeanrenaud, A.: Versteigerung aus der Prachtsammlung J. s. Auktionsliataloge. Koehler, Heinrich: Katalog der Briefmarken-Auktion $\mathrm{Nr} .4$.

Jeran, Osear: Wegweiser für Verkehrswertzeichen-Sammler (Philatelisten-Fibel). Berlin : Selbstverlag 1890, 144 S. $8^{\circ}$.

Inhalt: Kalendarium, Verzeichnis der PhilatelistenVereine u. d. philatel. Literatur, Preisangabe ron Raritäten $u$. folgende Fachartikel: Die Terwerthung vou Doubletten. - Vorgicht beim Ankauf von Verkehrewerthzeichen. - Einiges aus der Philatelie. Finigeg zur Geschichte der Verkehrswerthzeichen. Ist deu gebrauchten oder den ungehrauchten Marken der Vorzug zu gehen? - W'as gilt von Waserzeichen? Baggo, F. v.: Die kursiereudeo Ganzacluen Rusglands. - Das Hans Heitmann. - Biographien: liowland Hitl, Heinrich Stephan, J. Wilhelm, Freiherr Fon Dewez, Richard Krause, Wr. Alfred Mosehkau, Dr. Emanuel Herrmann, Victor Suppantschitseh, Paui Kloss, Theudor Haas, Georg Wendt (alias Kaufmanu), Ad. Schulze, Karl von Gindel. - Güudel, C. v. : Tie Chalmerg-Hill'sche Streitfrage.

liud. Heitmann, Ernst: Jhind: im rroben Handbuch der Philatelie" Bd. 2. \$. 325--334. Leipig 1889.

Indiens. Britisclı. Ustindien.

Indore. (Holkar.) Lindeuberg, C.: Holkar; im Groben Handouch der Philatelie ${ }^{*}$ Bd. 2. S. 208. Leipzig 1859.

Innerösterreichischer Philntelisten-Club s. Graz.

Internationale Postwerthzeichen-Ausslellung in Wiens. Friedl u. Baum.

- s. a. Wien.

Internationaler P'hilatelisten-Verein Iresden s. Diesden.

Johannes, Carl [d. i. Carl Joliannes Ścholtze|: Praktische Anleitung zum Briefmarken sammeln. Leipzig: Johannes Scholtze 1896. 100 S. S $^{\circ}$. [s. a. u. Sclioltze*.]

Johore. Lindenb ${ }^{2 r g}$, C.: Johore; im GroBen Handbuch der Philatelie" Bd. ¿. S. 337-3.39. Loipzig 1889.

Ionische Inseln. Lindenberg, C.: Ionische lnstln; im Großen Handbuch der Philatelie" Bd. 2 S. 239 u. 240. Leipzig 1889.

- Kohl, Paul: 1llustr. Katalog der Freimarken von Europa. Abt 3. "Ionische Inseln".

Joris, Cornelio: Aufstellung der Couverte, Streifbänder, Postkarten und Postanweisungen des Königreichs Baiern, München: Selbstverlag 1883. $22 \mathrm{~S} .8^{\circ}$. [Autographiert.]

Joris, Cornelio u. Otto Sedlmayr: Geschichte und Katalog del Postwertzeichen des Königrleichs Bajern. 2 Teile. München: Selbstverlag des Bayerischen Philatelistenvereins $1891-1893.8^{\circ}$.

Teil 1: Geschichte, $104 \mathrm{~S}$

$$
\text { 2: Katalog. S. } 105-108 .
$$

[Uñvollendet. Fracbien erstmalig als Beilage zum ${ }_{n}$ Postwerthzeichen" Jg. 1891-1893.]

Joseph, Dr. s. Bauschke, G.: Katalog allerbekannten seit 1840 bis Mitte 1865 emittirten Briefmarken. Leipzig 1865.

s. a. Zeclimeyer, G.: Der Briefmarkenhandel vor den Breslauer Gerichten .. Nürnberg 1888.

Joessel, Mlfous: Praktische Anleitung zum Briefmarken-Sammeln. [Umschl.]: Das Briefmarkensammeln. Leipzig: Verlag für Kunst und Wissenschaft Albert Otto Paul, o. J. 56 S. $8^{\circ}$. (Bd. 278 der Hiniatur-Bibliothek.)

- Die Postwertzeichen der OesterreichischUngarischen Monarchie. Anlüsslich des 50jïhrigen liegierungs .Jubiläums Seiner Kíaiserlichen u. Königlich-apostolischen Majestät Franz Josef 1. . . hrsg. von A.J. Leipzig: Scholtze \& Klemm 1898. IV, 242 S.m. Abb. $8^{\circ}$. 
Ioessel, Alfons: Vie Postwertzeichen der nesterreichisch-Ungarischen Monarchie. Neue ergänzte Ausg. Leipzig: Richard Scheffe] [1900|. II, $248 \mathrm{~S} . \mathrm{m}$. A bb. $8^{\circ}$.

Isakovies, Alois von: Ein Beitrag zur V'ereinsfrage; in: Isakovies, A.v.: Intern. 'I'ausch-, Adress- 11. Huilfsbuch f. d. 'Tauschverkehr. 'l'. 2.

- Internationales 'l'ausch-, Adress- und Hiilfsbucl fiu den Tauschverkehr. 2 Teile. thimn: Selbstverlag 1886. $8^{\circ}$.

Teil 1. Tauschadressen. S. 1-34.

2. Verschiedene Fachartikel. S.35-37.

Islaud. Heitmann, Ernst: Island; im firoben Handbuch der Philatelie Bd. 2. S. 241-244. Leipzig 1883.

Kohl, Paul: Illustr. liatalog der Freimarken von Huropa. Abt. 4. "Island".

- Ruben, H. E.: Voltstindige Preisliste von lsland .. Kopenhagen 1889;90.

- Willadt, Carl: Island, speziell I Gildi. Be. merkenswerte kurze Ausführungen zu dem ursprünglich rorgeselienen, aber nicht gelialtenen Vortrag des Dr. Bergmaun Hannover] aut lem XII. Dentschen Pbilatelisten'T'age . . . in Leipzig' (u. Halle a./S.) 1904.

Israel, Wilhelm Theodor s. Bauchke, Gustav: Katalog aller bekannten seit 1840 bis Mittp 1865 emittirten Briefmarken.

Ist den gebrauchten oder den uugebrauchten Marken der Vorzug zu geben? in: Jelan, O.: Wegweiser f. Verkehrswerthzeichensammler (Philatelisten-Fibel).

Ist es vom philatelistischen Standpunkte aus angehracht, Spezialsammlungen anzulegen, was ist in solche aufrumehmen $u$. wie verfährt man hei evtl. Anlage? Mähr.-Ostrau: Julius Kittl. 1887, $18 \mathrm{~S}, \mathrm{~S}^{\circ}$.

Italien. Borek, li.: Die Briefmarken der Italienischen Postämter in der Levante. Braunschweig 1908.

- Doczkalik, E.: Handbuch der MunicipalStempelmarken Italiens. Kaposvár 1911.

- Hummel, Sigmund: Katalog italienischer Militärmarken. München 1915.

Kalckhoff, Franz: Italien; im "Groben Handbuch der Philatelie ${ }^{*}$ Bd. 2 \$. 247--276. Leipzig 1889.

- Kirch, R.: Illustr. Katalog der Postwertzeichen... von Italien u. den ehemaligen italienischen Kleinstaaten ... Neapel [1894.]

Kohl, Paul: Illustr. Katalog der Freimarken von Europa. Abt. 7. Italien, Altitalienische itaaten.

Ravel, Wrnest, Johann: Illustr. Katalog der Postwerthzeichen... vou Italien u. den chema], italien. Kleinstaaten . . [1894.]

- Risigari, L.: Die Marken der italienischen Kleinstaaten. Vortrag geh. auf dem VIl. Deutschen Philatelisten-Tag zu Mannlieim 1895.
Jugenderziehung, Philatelistische. Vortrag geh. von Franz Balke s. Deutscher Philatelistentag; in: Stenograph. Bericht des XXI. Deutschen Philatelisten-T'uges... su Karts. bad 1909.

Jung, J., Entwickelung des deutschen Postund Telegraphenwesens iu den letzten 25 Jahıen. Gedenkblätter zum 25 jähr. Bestelren eines einheitlichen Post- u. 'Telegraphenwesens in Teutschland. \%weite unveränderte Auf. Leipzig: Duncker \& Ilumblot $18 \% 3$. 185. S. m. 7 graplischen 'Taf. 80 .

Der Weltpostvereiu und sein linfluss auf len Weltverkehr und die Weltwirtschaft. Hin Vortrag geb. in der Gesellseluaft f. Errl kunde u. Kolonialweseu \%u Strabiburg. Stralslourg: J. H. E. Hoitz 1903. IT, 46 今. п. 5 litlograple. Taf. $4^{0}\left(\mathrm{gl}^{\circ} .8^{\circ}\right)$.

- Ler Weltpostverein und der Wiener l'ostkongress. (Aus: Jalrbuch fiir lieset\%gebung etc. $\left.{ }^{*}\right)$ Leipzig: Huncker \& Humblot 1892. V, 58 S. m. 2 graph. Darstellungen. $4^{0}$ $\left(\mathrm{gl}^{\circ}, \mathrm{S}^{0}\right)$.

Jiirgens, Wollemar: Preisliste |Nr, 1. | llelsingsfors 1-77. 1 Bog. $2^{\circ}$.

Ni.2. Ebda. 1878.4 S. $4^{\circ}$.
- Nr.3.
- Nr. $4 . \quad$ "1875.] 31 S. 4".

- Katalog-Preisliste (Nr. 5) aller Marken, Karten u. Briefumschläge von limmland u. Russland, mit einem Anhange enthaltend: -Verschiedenes", April 1880 ... Hbda. 1880. 16 S. $8^{\circ}$.

- Engros-Preisliste (Nr. 6). Hbda. 1882. 89 s. $8^{\circ}$.

- Katalog-Preisliste (Nr. 7) . . Fbda. 1883. 32 S. 80 .

'ritel wie bei Nr. 5.]

- Ḱatalog.Preisliste (Nr. 8) aller Harken, liarten u. Briefumschläge von Finnland, Russland, Dänemark, Norwegen u. Sehwelen, mit einem Anhange enthaltend: "Verschiedenes ${ }^{*}$. Reval 1886, 52 S. $8^{\circ}$.

— - Nachtr. 1. Ebda. 1886. 4 s.. 8\%.

- Jager-Verzeichniss (Nr.9) (engros it en detail). Specialitat: Russland, Finnland u. Scandinavien der Postwerthzeichenhandlung von W. J. Ebda. 1891. 31 \$. $8^{\circ}$.

\section{K. K. Versteigerungsam Ihorotheum Wien} s. Auktions-Kataloge.

Kaiser, WiJhelm: Die Postscheine des Königreichs Hannover. Eine katalogische Zusammenstellung mit kurzen geschichtlichen Rüickblicken von IV. K. Göttingen. o. O. u. I. $31 \mathrm{~S} .4^{0}$.

[Wrschien erstmali. als Zeitungartikel in eiuzelneu Forketzungen im Jg. 1911 Der deutecho Philatelist, Verlag von Guslav Frejse, Hannorer.]

Kaisertum, Ottouanisches, s. Meyer, Ferl.: liatalog des Ottomanischen liaiserthums. 
Kalckhoff, Frauz: Ueber die Einteilung der Marken und über den Sammelwert der einzelnen Gattungen. Ein Beitrag zur Albumfrage. Vortrag s. Deutscher Philatelistentag; in: Stenograph. Bericht über den 5. D. Ph. in Berlin 1893

Die Erfindung der Postkarte und die Korrespondenzkarten der Norddentschen Bundes. post. Mit 2 Beilagen u. 18 Abb. im Text. Leipzig: Krötzsch \& Co. 1911. VI, 58 S. $8^{\circ}$. Grosses Handbuch der Plilatelie. Lfg. 2 u. 3 in zweiter Auf. Bearb. von Dr. K. Leipzig: Finst Heitmann $1890 / 91$.

[в. a. u. Teltz, O.: Grosese Handbuch der Plitatelie Lfg. $21-27$.

- Ueber Markenconservirung. Vortrag s. Deutscluer Philatelistentag; in: Stenograph. Bericht ï, d. Verhandlgu, des 6. Deutschen Philatelistentages in Kiel 1894.

- Die Postkarten der deutschen Schutzgebiete und der deutschen Postanstalten im Auslande. (Berichtigter u. ergänzter Sonderabdr. aus der Deutschen Briefm. Ztg.) Leipzig: Hugo Krötzsch 1902. $32 \mathrm{~S} .8^{\circ}$.

- Die T'elegraphenmarken und Fernsprechscheine der Deutschen Staaten. (Sonderabdr. aus der , Berliner Briefm.-Zeitung, Jg. 1910.) Berlin: Plilipp Kosack 1910. $8^{\circ}$.

Die Preussischen 'Telegraphen-Marken s. Berlin (Berliner Philatelisten-Klub) in: Festschrift z. Feier des zehnjährigen Restehens des B. Ph.-Kl, Berlin 1898.

Illustrirtes Verzeichniss aller bekannten Neudrucke staatlicher Postwertzeichen nebst Angabe ihrer Unterscheidungsmerkmale. Mit einer Einleitung von Landgerichtsrat Lindenberg. Unter Mitwirkung hervorragender Sammler u. Händler hrsg. von F. K. Leipzig : Ernst Heitmann 1892. 96 S. $8^{\circ}$.

- - Nachtrag. Ebda. 1893. 20 S. 8*.

- s. a. Berlin (Berliner Philatelisten-Klub). Festschrift zur Feier des zchnjäbrigen Be. stehens des B. Ph.-Kl. Berlin 1898.

Kalckhoff, Franz u. Ednard Sachse: Geschichte des Berliner Philatelisten.Klubs s. Berlin. (Berliner Philatelisten-Klub) in : Festschrift z. Feier des 25 jähr. Bestehens des B. Ph.-Kl. Berlin 1913.

Kalender für Briefmarkensammler s. Starke, Kionrad.

Kap der guten Hoflnung. Iloffmann, E.: Cap der guten Hoffnung; im Groken Handbuch (ler Philatelie" Bd.1. S.28I -294. Leipzig 1888.

Kap Verdische Inselı. Heitmann, Ernst: Cap Verde; im ,Großen Handbuch der Philatelie Bd. 1 S. 295-298. Loipzig 1888.

Kaschmir. Heitmann, Ernst: Kaschmir; im "Groben Handbuch der Philatelie" Bd. ". S. 342-354. Leipzig 1889.

- (Jammu.) Kohl,Paul: Die Marken von Kaschmix. Clemnitz 1911.

Kataloge s. Auktions-Kataloge; Postwertzeichen-Kataloge.
Katalogpreise, Ueber. s. Willadt, Carl. (Vortrag, gehalten auf dem XVIII. Deutschen Plilatelistentag in Nürnberg 1906.)

Kalechisuus des Briefmarkensammlers : Finke, freorg.

- s. a. Marbes, August.

Katscher, Leopold: Das Postwesen einst und jetzt. Mit 23 Jllustr. Berlin, Leipzig: IIermann Hillger Verlag [1906]. 94 S. $8^{\circ}$.

(Hillgers illustr. Volksbücher Bd. 68.)

- Der Weltpostverein. Gautzsch b. Leipzig: Felix Dietrich 1909. 14 S. 80.

Kauscl,, 0skar: Die Sprachwissenschaft in der Briefmarkenkunde, Ein Handbuch für Sammler jeder Richtung, insbesondere für Freunde des Briefmarkensammelwesens. Dresden: E. Stryk 1886. 184 S. 8\%.

[Hierron erschien auch ein $z$ weiter Abdruck in Krehn, Julius: Internationales Pbilatelisten-Adressbuch und ist mit diegem zusammengebunden ]

- Zweite verbess. Aufl. Leipzig: W. A. Louis Senf \& Co. 1891.11, 243 S. $8^{\circ}$.

(Bibliothek für Postwerthzcichen-Ssmuler Bd. 4.)

Dritte durch Nachträge erweiterte Aufl. Leipzig: Gebrüder S'enf [1895]. VI, 248 S. 9". Kurze Inhaltsangalo:

Vorwort.

1. Snrachwise enschaftl. u. literarischoVorbemorkungou.

2. Die germanischen u. romanischen Sprachen auf dov Marken: a) deutsch, L) onglisch, c) holländisch, d) dänisch, e) schwediech, f) itslienisch, g) fran zD̈вi

3. Die Aussprache der Msrken-Inschriften. a) Allgemeinse, h) englisch, c) holländisch, d) dänisch, e) schwodisch, f) italienisch, g) französisch, h) вpsnisch, i) portugiesisch, k) rumänisch.

4. Darstellung u. Auseprache slaviooher, magyarischer, griechischer, eanscrita und persischer luschriften: a) russisch (mit Darstellung der ruse. Druckschrift), b) polnisch, c) tschechisch (böhmisch), d) msgyarisch (ungarisch), o) griechisch (neugriechisch) mit Daratellung grischischer Schriftzetchen, f) sanskrit mit Darstellung dor Dêvanagirischrift, g) persisel mit Daretellung der arabisch-peraischen Schrift.

5. Wörterbuch der Brisfmsrkenkunds.

6. Nachträge.

- Der Wechsel in den Vorbildern der Postwerthzeichen; in: Senf, Gebr., Taschenbuclı f. Briefmarken.Sammler pro 1889.

Orientalische Zahlenzeichen. (Festgabe zum 10. Stiftungsfest des Internationalen Philatelisten-Vereins Dresden.) Dresden: Ernst Petritz [1887] Taf. $2^{\circ}$ in Mappe $12>16 \mathrm{~cm}$. [Litlogrsphiert.]

- Die Namenkunde der Länder u. Städte des Deutschen Reiches. Leipzig: Frnst Heit mann 1890. VIII, 219 S. $8^{\circ}$.

Kees, Johann Jakol I u. II. Oberpostmeister s. Kieus, Kurt: Das kursächsische Postwesen.

KeesJacher, Carl: Oesterreichisches Postwesen. Hine Zusammenstellung aller noch giltigen Verordnungen aus den 33 bisher erschienouen Verordnungs-Bänden. Von C. K., k. k. Postamtsverwalter in Villach. Klagenfurt 1870. 2 Teile. 80.

T. 1. XXXVIII, 656 \&.

T. 2. S. $657-1159$. 
Keitel, Otto: Feldpost-Ratgeber beim Verschicken u. Verpacken von Feldpostsendungen mit einem Wörterverzeichnis über die gebräuchlichsten militärischen Bezeichnungen. Leipzig: E. Stoll 1914. $12 \mathrm{~S} .8^{\circ}$.

Keller, Anton: Statistische 'l'afel mit vel' schiedenen Vergleichstabellen zsgest. f'ür Freunde der Briefmarken-liunde. Von A. I. k. b. Post-Revisor. Im Juli 18S4. Münclien: Selbstverlag] 1884. 25 S. $4^{\circ}$.

[Lithograpliert.]

Kiautschou. [Friedemann, Albert]: Die Aushülfsmarken rou Tsingtau u. ihre Fälschungen. Leipzig 1903.

Kiel. (Kieler Philatelisten-Kilub.) ErinnerungsBlatt für die Theilnehmer an der 25 jährigen Stiftungsfeier des K. Ph.-Kl. am 8. Dezember 1900. Kiel: Selbstverl. 1900. 16 S. $8^{\circ}$

Kirch, Rollevich: lllustrierter Katalog der l'ostwertzeichen (Bricfmarken, Postkarten, Kartenbriefe, Geldanweisungen, Postpaketbegleitharten) von ltalien u. deu ehemaligen italienischen Kleinstaten mit Preisverzeichniss der Briefmarkenhandlung R. K. Neapel: [Selbstverl. 1894.] $42 \mathrm{~S} .8^{\circ}\left(16^{\circ}\right)$,

[s. a. Rarel, Ernest Johau»" unter dem gleichlautenden Titel gedr.]

Kirchenstaat. Diena, Emilio: Die I BajoccoMarke des Kirchenstaates. Berlin 1898.

- Glasewald, A. E.: 'l'afel mit den verschiedenen Neudrucken von Kirchenstant. GöBnitz 1910.

- Lindenberg, C.: Kirchenstaat; im ,GroBen Handbuch der Philatelie* Bd. 2. S. 355-360. Leipzig 1889.

- Rom 1 Scudo u. 50 Bajocci (Fälschungen); in: Moschkau, A., Zur Geschichte der Philatelie.

hirchhofer, Hans s. Reuterskiöld, A. de: Die Kantonalmarken der Schweiz und deren Fälschungen

Kirmis, M. s, Klasing, Otto: Das Buclı der Sammlungen". 6. Aut. 1906.

Klasing, 0tto: Das Buch der Sammlungen. Praktische Anleitung zum Anlegen, Ordnen und Erhalten aller Arten von Sammlungen, überhaupt zur Unterstützung natur- u. geschichtswissenschaftlicher Liebhabereien. Unter Mitwirkung bewährter Fachmänner für die deutsche Jugend hrsg. 5. vermehrte u. verbesserte Autl. Bielefeld u. Leipzig: Velhagen \& Klasing 1890. 458 S. $8^{\circ}$.

(Artikel : "Die Postwertzeichengammluug ", S. 425 bi 458.)

- 6. völlig neubearbeitete Aufl. von Prot. Dr. MI. Kirmis, Ebda. 1906. 422 S. $8^{\circ}$.

(Artikel : "Die Postwertzeichensammlung", S. 391 bis 422.)

Klaus, 0.: Die Post aut dem Weltmeer. Berlin: F. S. Mittler \& Sohn. 1908. 39 S. m. $\mathrm{Abb}, 8^{\circ}$.

(Meeregkunde. Sammluug volketïmlicher Vorträge, Jg. 2, H, 9.) hleeberg, Paul: China. Seine Post und scine Freimarken. 1878-1910. Chemnitz: Paul Kohl G. m. b. H. 1911. VII, 63 S. m. Abb. u. 4 Taf. i. Text. $8^{\circ}$

- Katalog der Marken der KíaiserJicben Post in China. (Pei)age zu: Kleeberg, Paul: China, seine Post u. seine Freimarken.) Ebda. 1911. $16 \mathrm{~S} .8^{\circ}$.

Klenze, Iteinricl rou: Referat betr. Agitation gegen das Aufdruckunwesen. Hünchen: Bayerischer Philatelisten-Verein 1885. $4 \mathrm{~S}$. Q

[Autographiert.]

Referat betr. Reform der Vordruckalben. München: Ebda. 1885. $4 \mathrm{~S} .8^{\circ}$. [Autographiert.]

- Die Ziele der Briefmarkenkunde; in: Isakovics, A. v.: Internationales Tausch-, Adressu. Hïlfsbuch für den Tauschverkehr. T. 2.

Kliewes, P.: Manco-Liste fül Jen 'Tausch. Verkelir. Danzig: Selbstver]. [I880]. $38 \mathrm{~S}$. $8^{\circ}\left(16^{\circ}\right)$.

- Preiskatalog der internationalen Briefmarkenhandlung P. Kl. Danzig: Selbstrerl. [1876]. 64 S. 8".

- Nachtr. Ebda. 1878. 1V, 16 S. $8^{\circ}$.

[Bildet zugleich die 2. Aufl, des 1876 ergchicnenen Hauptwerke日.]

— - 3. Aufl. Ebda. [1880]. IV, 50 s. $4^{\circ}$.

Kloss, I'aul: Biographie; in: Jeran, O.: Wegweiser für Verkebrswertzeichen-Sammler.

- Geschichte der Post-WVerthzeichen des Kö. nigreichs Sachsen. Mit 68 11l. Nach amtl. Quellen bearb. Leipzig: Gebr. Senf (1882). 85 S. $8^{\circ}$.

- Nachträge zu Meyer's Handbuch für Postmarken-Sammler. $8^{\circ}$. Niirnberg: ${ }^{\circ}$. Zechmeyer.

$$
\begin{array}{ccc}
\text { Nacbtr. } & 4 . & 1884 . \\
\pi & 5 . & 1886 . \\
& 6 . & 1888 . \\
& 7 . & 1890 .
\end{array}
$$

- Streitfrage "Chalmers contra Hill" s. Deutscher Philatelistentag Antrag 6; in: Bericlit ii. d. ... Il. Deutschen Philatelisten-Tag zu Frankfurt a. M. 1890.

- Veseinigte Staaten von Nord-Amerika. Eint Couvert- und Streifband-Aufstellung (615 Nummern). Leipzig: Louis Senf I880. $32 \mathrm{~S}$. m. $26 \mathrm{Abb} .8^{\circ}$.

(Philatelistieche Bihliothek Bd. 5.)

- Verzeichniss u. Beschreibung aller PostKarten mit aufgedruckten Werthstempe]. Bearb. von Dr. jur. P. K. Tresden: Alwin Nieske 1880. 297 S. $8^{\circ}$.

[Die Seiten 3-176 waren ursprünglich in einzelnet Teilen als Ergänzmng zur Union": Dentache Brief - warken-Zeitung, Jg. 1879 veröffentlicht. 8. a. u. Dre日den (Internationaler Philatelisten-Verein) Verzeichnis Ci $11,8, \mathbf{w}$.

- - Zweite Ausgabe. Nit Nachträgen. Dresden: Ernst Petritz 1882. 310 S. $8^{\circ}$.

- s. a. Moschkau, A.: Handbuch f. Postmarmarken-Sammler. Nachtr. 5. Leipzig 1886. 
Filuss, Hul: s. a. l'etritz, Elust: Manco-Liste zu Dr. Kloss's harten-Catalog. Dresden 1885.

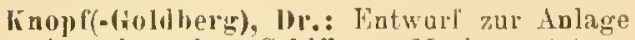
einel deutschen Schiffs- $u$. Marinepoststempel Sammlung. (Gonderabdr. aus .Mitteldtsch. Philatelistenztg.") Göfaitz: Glasewald 1901. $8 \mathrm{~S} .8^{\circ}$

linorr, Louis: Markenmüller. Philatelistische Berichte. Leiprig: Gebrider Senf [1892]. $1325.8^{\circ}$.

Kunst, Jnlius: Illustrierter Kinkaufs-Katalog über Altdeutsche Marken 1894/95. Versehen mit 53 Marken-Abb. ฉ. 624 Einkaufspreisen der Briefmarkenbandlung .I. is IIarburg a. d. Elbe 1894. $20 \mathrm{~S} .8^{\circ}$.

forlu. Feorg: Kiatalog der auf der Internationalen Postwertzeichen-Ausstellung zu Berlin 1904 aufgelegten Sammlungen von G. K. Giessen. Berlin: [A usstellungsleitung] 1904. $22 \mathrm{~S} 8^{\circ}$.

Inhalt: Deutschea Reich u. seine liolonien, Alt deutsclie Staaten, Ganze Bogen.

Internationale Postwerthzeichen-Ausstellung Wien 1890. Verzeichniss der von Georg Koch in Cairo ausgestellten Administrationsmarken Aegyptens. Kairo, Wien: [Selbstver]. 1890. 18 S. $8^{\circ}$.

s. a. Merläuder, Ludwig: Die Administrationsmarken Aegyptens. Alexandria, Leipzig 1890.

liohl, Paul: P. K.'s Briefmarken-Handbuch und Grosser Katalog 1912. 9. Aufl. 2 Bile. Leinw. geb. Chemnitz: Panl liohl G. m. b. H. 1912. $8^{\circ}$.

Bd. 1. Europa mit allen Kolonien europäisiher Staaten. XXXl, 1258 S.

Bd. 2. Selbständige Ueberseestaaten. XVI, $560 \mathrm{~s}$.

1915. 10. Aufl. (Jubiläums-Ausgabe.)

2 Bde. Leinw, geb. Hbda. 1915. 8.

Bd. 1. Europa mit allen Kolonien euro. päischer Stanten. XXX11, 1536 ฬ..

Hid. 2. Selbständige L'eberseestaaten. XVI, $675 \mathrm{~S}$

- P. K.'s illustrierter Briefmarken-Refurıliatalor 1907. Chemnitz: Paul Kohl G. m. b. H. 1907. IVI, $830 \mathrm{~S}$. Leinw. geb. $8^{\circ}$.

- - 1908. Fbda. 1908. XVl, $872 \mathrm{~S}$. Leinw. geb. $8^{\prime \prime}$.

[Fortsetz, s. u. d. T. "Briefmarken-Norma]-Katalog, IIlustrierter.".]

- Freimarken Katalog 1900. Hrsg, von P. K. Europa, Europäische Kolonien, Ausland. 2 Bde. Chemnilz: Paul Kobl. 1900/01. 8".

Bd. 1. $216 \mathrm{~S}$.

Bd. 2. $304 \mathrm{~S}$.

[Titel von Bd. 2 lautet : "Freimarken-Katalog $1900 / 01^{\prime "}$. Das Gesamtwerk bildet die 1. Autl, von Kobl's Grosser Katalog u, Briefmarken-Handbuch.]

- - [Zweite verbesserte Auflage, mit allen Narken Abbildungen u. Aufdrucktypen 1902.7 Ebda. $1902.736 \mathrm{~S} .80$.

- Freimarken-Katalog 1902-1903. [3. Aufl.] Fbda. [1902]. XXX1], 834 S. 8.

- Freimarken-Katalog 1903-1904. [4. Auf.]

libda. [1903]. XXIV,976 S. $8^{\circ}$.
Kolsl, Panl: Wreimarken-Katalog 1904-1905. [5. Aufl.] Hbda. [1904]. XXIV, 1112S. $8^{\circ}$.

- 1906. Freimarken-Katalog. 6. Aufl. Ebda. [1905] XXIV, 1152 S. 80.

[Fortsetz. 8. u. d. T': ${ }_{n}$ Kolil's 1llnstrierter Briefmarken-Reform-Katalog " sowie auch "Kohl's Grosser Katalog "1. Jriefmarken-Handbuch" ${ }^{\text {. }}$.]

P. K.'s illustrierter Freimarken-liatalog Englische Kolonien. 3 Teile. Chemnitz: Paul Kohl 1898-99. $8^{\circ}$.

Abt. 1. Kolonien in Nordamerika u. Westindien. $60 \mathrm{~S}, 1 \mathrm{n}$. Abb

Abt. 2. Kolonien in Afrika. 48 S. m. Abb.

Abt. 3. Kolonien in Australien nebst Hawai, Samoa, Tonga... Bearb. von William Uhlich. 67 S. m. Abb.

- P. K.'s Grosser Tratalog und BriefmarkenHandbuch 1907. 7. Auf. Chemnitz: Paul kiohl 1907. XXIV, 1256 S. m. Abb. Lninw. geb. $8^{\circ}$.

- - 1909. 8. Auf. Fbda. 1908. XXIV, 1522S. м. Abb. Leinw. geb. $8^{\circ}$.

Berichtigungen u. Nachträge zu Kohl's Grassem illustrierten Káalog u. Handkucl 1909. Ebula. [1908]. 3. S. $8^{\circ}$.

- P. I.' illustrierter Ḱatalog der Freimarken Deutschlands, enthaltend sämtl. Freimarken des Dentschen Reichs $u$. der Deutschen Stanten u.s. W. Chemnitz: Paul liohl 1892. 46 S. m. Abb. $8^{\circ}$.

- 2. Aufl. Ebda. 1894-1895. 63 S. m. Abb. $S^{0}$

[Sonderausg, von Paul Kohls illustr. Kata]og der Freimarken ron Europa. Abt. 1.]

- l'K.'s illustrierter Katalog der Freimarken von Deutschland... 3. stark verm. Aufl. Ebda. 1897. $70 \mathrm{~S} .8^{\circ}$.

- I. K.'s illustrierter Katalog der Freimarken von Furopa. In 9 Abteilungen. Chemnitz: Paul Tíohl 1894-95. 8".

Abt. 1. Deutschland, 2. Aufl. $63 \mathrm{~S}$.

Abt. 2. Belgien, Frankreich, Luxemturg, Niederlande. 39 S.

Abt. 3. Grossbritannien, Gibraltar, Helgoland, lonische Inseln, Malta. $27 \mathrm{~S}$.

Abt. 4. Dänemark, Island, Russland, Finnland, Polen, Schweden, Norwegen. $35 \mathrm{~S}$. Abt. 5. Oesterreich-Ungarn, Bosnien. $31 \mathrm{~s}$. Abt. 6. Schweiz. $23 \mathrm{~S}$.

Abt 7. Italien, Altitalienische Staaten, San Marino, Monaco. $33 \mathrm{~S}$.

Abt. 8. Balkanstaaten u. Griechenland. $62 \mathrm{~S}$.

Abt. 9. Spanien u. Portugal. 63 S.

P. K.'s illustrierter Katalog der Freimarken von Europa. Praktische Vorlage für Paul Kohls Permanent-Briefmarken-Album. 2. stark verm. Aufl. Ebda. 1897. 424 S. $8^{\circ}$.

- Katalog der Kriegsmarken von Deutschland, Oesterreich-Ungarn und den neutralen Staten, sowie verschiedener durch den Krieg hervorgerufener Marken. Chemnitz: Paul Koh] [1916]. 12 S. 8".

[Dieser Katalog ist aus den beiden Naclitrïgen zum Neuen Normalkatalug 1915 entstanden.] 
Kolıl, Iaul: Die Marken von Kaschnir (Jammu), mit vielen Abbildungen und 6 'l'ypentafeln. Chemnitz: Paul Kohl G. m. 1. 11. 1911. 30.5. $8^{\circ}$.

- Offerten-Tafel Nr. 1-12. $8^{\circ}$.

[Wiedergabe seltener postwertzeichen suf kumst. drucktafeln nebst erlihuterndem Text.]

- Die Postwertzeichen von Aethiopien (Abessinien). Chemnitz: Paul Kohl G. m. b. H. 1911. $16 \mathrm{~S}$, m. Abb. u. 5 Taf. $8^{\circ}$.

- Die Postwertzeichen von Papua. Kataloy u. eingehende Beschreibung. Chemnitz: Paul KohI G.m.b.H. 1912.48 S.m.Abb.u. I'af. $8^{\circ}$.

[Den Seiten $35-48$ eteht eil besonderes Titelblatt vorau: "Katalog der in Britiech-Neuguinea epäter Papua postreitig veraugabten Marken."

- Probedrucke, Marken mit Specimen sowie Essais der Vereinigten Staaten von Amerika. Chemnitz: Paul Kohl G. m. b. H. 1911. 40 S. m. Abb. $8^{\circ}$.

- Typentafeln. Chemnitz: Paul Kohl o.J. $8^{\circ}$. Bamra 1889, Originale.

San Marino, 'Typentafeln zu Katalog Nr.8, 9,10

San Marino, in OriginalgröBe (Photographie).

Rumänien, in OriginalgröBe (Photographie) Nr. 22 I.

Rumänien, Moldau 81 Parale, Original u. Probedruck, stark vergröBert.

Rumänien, Moldau 108 Parale, Original u. Probedruck, stark vergröBert.

Schweiz, Typentafeln zu Katalog Nr. 10 (Photogr.).

Turks-Inseln, Typentateln zu Katalog Nr. 7-17 (Photogr.).

Victoria, Typentafeln zu Katalog Nr. 2 A u. 2 B (Photogr.).

Vorzugs-Angebot seltener Marken. Chem. nitz: Paul Kohl G. m. b. H. [1916], 8.

(Liste) 35. Afghanistan bis Bulgajien. S. $1-66$.

Liste 36. Canada bis Franz.-Indien. S. $67-130$.

Liste 37. Franz-Indochina bis Helgoland. S. $131-194$.

Liste 38. Helgoland bis Kreta. S. 195-260.

Liste 39. Labuan bis Mlexiko. S $261-324$.

Liste 40. Noçambique bis Nicaragua. S. $325-388$.

Liste 41. Niederlande bis Pahang. S. $389-452$

Liste 42. Panama bis Portug.-Indien. S. $453-516$.

Liste 43. Portug.-Kongo his Salvador. S. $517-580$.

[Weitere Liaten ergcheinen, Das Ganze gibt ein geschlossenes Werkchen.]

Köhler, lleiurich: [Katalog der] BriefmarkenAuktion $\mathrm{Nl}$. 12. Stïckweise Versteigerung einer Prachtsammlung im Küustlerhaus Berlin W., Bellevuest. 3. Versteigerungstage: 20. u. 21. JX. 1916. Berlin: Heinrich Köhler 1916. 58 S. m. 1 Taf. u. 4 Abb. im Text u. 12 Taf. m. Abb. apart. $8^{\circ}$.

[Die vorhergehenden Kataloge Nr. 1-11 s. unter "Auktions-Kataloge".]

Ton, Handbuch.
Kibler, Heinrich: [Katalog dor] BriefmarkenAuktion (Nr. 13). Stïckwoise Versteigerung einer Prachtsammlung von Marken auf Brief im Künstlerhaus Berlin W., Bellevuestr. 3. Versteigerungs-Tage:28.-30.Novbr. 1916. Berlin: Heinrich Köller 1916. 48 S. m. 26 Abb. im 'l'ext 11. 12 Kunsturuck 'Tafeln. $8^{\circ}$.

- Preisliste und Gelegenheits-Ofterten vorrätiger Briefmarken, Blockstücke, Marken auf Brief, Ganzsachen nebst Angeboten von ganzen Sammlungen der Briefmarkenbandlung H. K. Berlin: Heinrich Köhler 1915. 68 S. u. 12 Taf. m. Abb. $8^{\circ}$.

Kolumbien. Michelsen, G.: Ueber die Postwerthzeichen der Vereinigten Staaten von Columbien. Vortrag, geh. auf dem 9. Deutschen l'hilatelistentag in IIamburg 1897.

Kommune-Post s. Zennig, Karl.

Konföderierte Staaten vou Amerika. Rechert, Joseph: Conföderierte Staaten von Amerika im GroBen Handbuch der Philatelie" Bd. 1. S. 387-392. Leipzig 1888.

Kongo-Staat. Hoffmann, E.: Congo-Staat im (iroBen Handbuch der Philatelie ${ }^{\star}$ Bd. 1. S. 393 - 397. Leipzig 1888.

- Sauer, K. C.: Permanenter BriefmarkenKatalog. Beigien und Congo-Stat. Mährisch-Ostrau [1889].

König, Bruuo Fmil s. Crole, B. F.

Köıig, Emil : Deutschlands Feldpost. Fin Gedenkblatt an den Deutsch-Französischen Kirieg 1870-71. Von E. İ., Herausgeber der "Deutschen Post" in Berlin. Nit einer Feld. postkarte. Gera: Issleib \& Rietzschel 1871. 61 S. 8".

- Geschichte der Briefgeheimnis-Verletzungen und des Cabinet noir in Frankreich, nebst einer kurzgefassten Geschichte der Briefverschlussmittel. Leipzig: W. Opetz 1881. $71 \mathrm{~S} .8^{\circ}$.

- Schwarze Kabinette. Mit Anlagen: Geschichte der Thurn und 'Taxis'schen Postanstalt und des österreichischen Postwesens. Ueber die gerichtliche Beschlagnahme von Postsendungen in Preussen-Deutschland. Von E. K., Begriunder der Wochenschrift „Deutsche Post ${ }^{*}$... Braunschweig: IV. Bracke Jr. 1875, $103 \mathrm{~S} .8^{\circ}$.

König, Lndwig: Philatelistischer Bericht über die Zeit vom XX. bis zum XXJ. Deutschen Philatelisten-Tago. Vortrag. s. Deutscher Philatelistentag; in: Stenograph. Bericht des XXl. D. Ph. . . z zis Karlsbad 1909.

- Bericht ïber philatelistische Veröffentlichungen in Tageszeitungen u. Familienjournalen. Vortrag. s. Deutscher Philatelistentag; in: Stenogr. Bericht über d. 5. D. Ph. in Berlin 1893

- Ueber philatelistische Literatur <mit Vorlagen). Vortrag. s. Deutscher Philatelistentag; in: Stenograph. Bericht ü. d. XVII. Deutschen Philatelistentag ... in Regensburg 1905. 
König, Ludwig: Ueber Vereinswesen. Vortrag. s. Deutscher Philatelistentag; in: Stenogr. Bericht ü. d. XllI. D. Ph. zu Berlin 1901.

Koeppe, Oskar 8. Larisch, A.: Nummern-Liste zum Preisverzeichnis von A. L., zu O. Koeppe's Permanent-Album etc. München 1892.

- s. a. Larisch, A.: Preisverzeichniss sämmtl. bis 1886 ersch. Postmarken, zugleich MancoListe zu Koeppes Permanent-Album etc. [1.-6. Auf.] Mlünchen 1887-1893.

Koren. Heitmann, Frnst: Korea im Großen Handbuch' der Philatelie" Bd. 2. S. 361-362. Leipzig 1889.

Kosack, Philipp: Berliner Briefmarken-Kata. log. Berlin: Philipp Kosack. $8^{\circ}$.

Ausg. 1909.[1908.]'T.1. Europa.II, 160 S.m.Abb. - 1909. T.2. Asien. II, 126 S. m. Abb.

"1910/11. T.3. Afrika, II, $6 \pm$ S. m. Abb.

- 1914. T.4. Amerika. 48 S. m. Abb.

- Europa. NNeue Ausg. m. d. Neben-Titeln: Gelegenheits-Augebote, 1llustr. Satzpreisliste, Deutsche Kolonialmarken, Sammlungen aus Privatbesitz, Seltenheiten usw.] Berlin: Philipp Kosack \& Co. $8^{\circ}$.

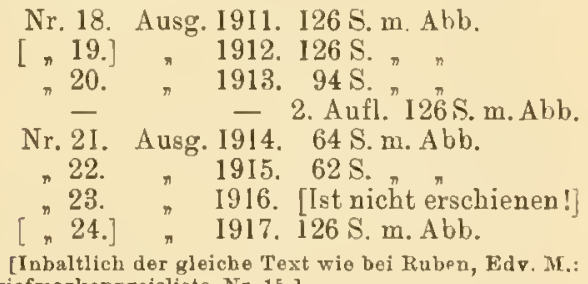
Briefmarkenpreisliate Nr.15.]

- Philatelistisches Feigenblatt 1Il: Zur Vertretung von Sammlerinteressen. Filzpariser oder Bismarck'sche Kürassierstiefel, dif einem „ausgewachsenen Philatelisten" nicht passen... P. Ohrt nachempfunden. Philatelist. Misch-satirische Monographie ... Berlin: Selbstverl. 1908. 24 S. $4^{\circ}$.

Kraiu. K. k. Post s. Radies, P. v.

Krasemann, Rudolf: Bibliographie der wichtigsten Spezialwerke über die Postwertzeichen einzelner Länder. Bearb. von $R$. K. Hrsg. von Sveriges Filatelist-Förening, Stockholm. Stockbolm: Sveriges-Filatelist-Förening 1907. $45 \mathrm{~S}$. Durchschossen. $8^{\circ}$.

- Zweiter durchgesehener u. stark verbesserter Abdruck. Sonderdr. aus "Der deutsche Philatelist" 1908. Hannover: Der deutsche Philatelist (Gustar Freyse) 1908. IV, 40 S. $8^{\circ}$.

[Wurde nor in $100 \mathrm{Expl}$. für den Privatgebrauch des Verfassers gedruckt.]

- s. a. Djurling, Hilmer u. Rudolf Krasemann: Die Postmarken von Schweden 1855 bis 1905 .

Krause, Richard: Biographie; in: Jeran, O.: Wegweiser für Verkehrswertzeichen-Sammler.

-Ueber Essais-Sammeln im Allgemeinen u. die deutschon Levante Essais im Besonderen; in: Isatovics, A. v.: Intern. Tausch-, Adressu. Hülfsbuch f. d. Tauschverkehr. T. 2.
K 1'ause, Riehard: Lehrbuch der Philatelie. Leip. zig: Ernst Heitmann 1889. XVIII, 144 S. $8^{\circ}$.

- Die Philatelie in Polen; in: Philatelia, Vereinsmittheilung der "Philatelia*. Sondershausen 1887.

Krebs, Kurt: 'Das kursächsische Postwesen zur Zeit der Oberpostmeister Johann Jakob Kees I u. II. Mit 4 Abb. im Text, 7 Taf., 2 farb. Porträts u. 3 mehrfarb. Postverkehrskarten. Leipzig: B. G. Teubner 19I4. XI, 566 S. $4^{0}$ (gr. $\left.8^{\circ}\right)$.

(Geschichte der Familie Kees. In Verbindg. m. Dr. Paul Kees u. Kurt Krebs brsg. v, Dr. Herm. Kees. Bd. 2.)

Krehn, Julius u. Max Löwinger: Internationales Philatelisten-Adressbuch. Wien: Sigmund Friedl 1882, $243 \mathrm{~S} .8^{\circ}$.

- Nachtrag 1 (Supplement) zum Internatiomalen Philatelisten-Adressbuch. Ebda. I884. 42 S. $8^{0}$.

- s. a. Reitz Edler von Bollheim: Das Haupt der deutschen Philatelisten.

Kreitz, CarI: Preisliste für Briefmarken-Sätze s. A bt. "Zeitschriften".

Kreta. Borek, Richard: Die Kreta-Mlarken mit dem "Ellas"-Aufdruck in Buchdruck. Braunschweig I909.

- Kutscha, Franz: Die Hellasaufdrucke auf den Marken von Kreta, deren Fälschungen u. Fälscher. Vortrag, geh. auf dem XXJ. Deutschen Philatelistentag zu Karlsbad 1909.

Kreuchauf, 01to: 100 Adressen von Briefmarkenhändlern [aus allen Weltteilen]. Hamburg: Selbstverlag 1872. Einblattdruck $4^{\circ}$.

Krenz, Unterm Eisernen 1914/15. Kriegsschriften des Kaiser-Wilhelm-Dank. Verein der Soldatenfreunde. H. 24. Große, O.: Die deutsche Feldpost. Berlin: Verlag Kameradschaft (1915) 64 S. $8^{\circ}$.

Krickeberg, E.: Heinrich von Stephan. Ein Lebensbild. Dresden u. Leipzig: H. Seemann Nf. 1897. 320 S. w. I Bildnis. $8^{\circ}$. (Männer der Zeit 1.)

Kriegsmarken. Kohl, Paul: Katalog der Kriegsmarken von Deutschland, Oesterreich-Ungarn usw. Chemnitz [1916].

- Locher, Eduard: Spezial-Katalog sämtlicher Rotkreuz- u. Kriegsmarken 1914/16. Zürich 1916.

Kropf, Hans: Die Abetempelungen der Marken von Oesterreich-Ungarn u. Tombardei-Venetien von 1850-1858. Prag: Franz Duschek. 1899. 162 S. u. 71 'Taf. m. 959 StempelTypen. $8^{\circ}$.

- Die Postwertzeichen der Oesterreich.-ungar. Monarchie, unter Benützung amtl. Quellen, bearbeitet von H. K. Als Jubiläumswerk des 50 jährigen Bestandes der Briefmarke in Oesterreich hrsg. vom Deutschen Verein für Briefmarkenkunde in Prag. (Enthält: Oesterreich, Lombardei-Venetien, Ungarn, Bosnien u. Herzegovina.) Prag: Deutscher Verein für Briefmarkenkunde. 1902. XIV, 455 S. m. 938 Abb. $4^{0}$. 
Kropf, Hans: Die Postwertzeichen des Kaisertumes Osterreich und der österreichisch-ungarischen Monarchie. Als Jubiläumswerk zur Feior des sechzigjähr. Regierungs Jubiläums Sr. Maj. des Kaisers Franz Joseph I. mit Benützung amtl. Quellen bearb. u. hrsg. von H. K. Mlit 35 Lichtdrucktafeln u. 34 Abb. im Text. Prag: Hugo Neugelaner, 1908. XIV, 447 S. 4".

Krötzsch, Hugo: Bericht über die Neudruckfrage. Vortrag. s. Deutscher Philatelistentag; in: Stenograph. Bericht des XXI. Deutschen Philatelisten-Tages . . zu Karlsbad 1909.

- Hirschfeld'sche Entwürfe u. Druckproben zu den Sächsischen Postfreimarken, s. Berlin (Berliner Philatelisten-Klub) in: Festschrift z. Feier des zehnjähr. Bestehens des B. Ph.-KI. Berlin 1898.

- Permanentes Handbuch der Postfreimarkenkunde mit Lichtdrucktafeln (und vierteljäbr. lichen Nachträgen), gleichzeitig Beibuch zum Pelmanent-Sammelwerk in losen Blättern. Ausführliche Abhandlungen über Postfreimarken mit besonderer Berücksichtigung del Herstellungsweise, Echtheitsmerkmale, Neudrucke, Fälschungen, Entwertungen und deren Fälschungen. Frster Teil: "Deutsche Staaten." Leipzig: Hugo Krötzsch 1893 bis 1897. So.

Bd. 1. Rommel, Otto: Deutsche Reichspost. 1893. 14 S. 口. 2 Taf,

Bd. 2. - Baden. 1893. 12 S. u. 1 Taf. Bd. 3. - Bayern. 1893 16 S. u. 2 Taf. Bd. 4. - Bergedorf. 1. Aufl. 1893. $10 \mathrm{~S}$. u. 1 Taf.

- Krötzsch, Hugo: Bergedorf 2. Aufl. 1896. 169 S. u. 9 Taf.

Bd. 5. Fommel, Otto a. H. Krötzsch: Braunschweig. 1893. 14 S. u. 4 Taf.

Bd. 6. - Bremen. 1893. 14 S. u. 6 Taf.

Bd. 7. - Hamburg. 1893. 20 S. u. 10 Taf.

Bd. 8. Krötzsch, Hugo: Hannover. 1593. 22 S. u. 9 Taf.

Bd. 9. - Lübeck. 1893. 24 S. u. 7 Taf.

Bd. 10. - Necklenburg-Scbwerin. 1. Aufl. 1893. 8 S. u. 7 Taf.

- Meckleuburg-Schwerin u. Mecklenhurg-Strelitz. 2. Aufl. 1895. $52 \mathrm{~S}$. и. 17 Taf.

Bd. II. - Norddeutscher Postbezirk <nebst Feldpost 1870/7I). 1894. 139 S. и. 21 Taf. nebst Karte der deutschen Poststralen in Frankreich 1870/71.

- 2. ergänzte Aufl. 1895.

Bd. 12. Ohrt, Paul: Oldenburg. 1894. 120 S. u. 18 Taf.

Bd. 13. - Preußen. 1896. 232 S. u. 14 Taf. Bd. 14. Sachsen. [Ist noch nicht erschienen.]

Bd. 15. Rosenkranz, A.: Schleswig-Holstein. 1897138 S. u. 14 Taf.

[Bd. 1-3, 4 (1. Aufl.) บ. 5-9 Bind als kurze Abbchnitte zu einem Sammelband vereinigt, dagegen bilden Bd. 4 (2. Aufl.) u. 10-15 selbotündige in sich abgeschlossene Einzelbande. Das Geramtwerk erachien iberdies erstmals 1893 in losen Bogen unter dem Titel: ${ }_{\pi}$ Permanentes Beibuch mit Ijichtdrucktafeln (u. vierteljabrl. Nachtr.) zum philatelıtischeu Permanent-Sammelwerk".]
Krötzseb, Hugo: Permanentes Ilandluch der Postfreimarkenkunde. Vierteljahrs-Nachträge. Mit 19 Lichtdrucktafeln. Ebda. 1894-1896. 204 S. $8^{0}$.

Jg. 1. Neujahr, Ostern, Johanuis, Michaelis 1894. Nr. I 4 .

Jg. 2. Neujahr, Ostern, Johannis, Michaelis 189j. Nr. 5-8.

Jg. 3. Neujahr, Pfingsten, Johannis, Weih. nachten 1S96. Nr. 9-12.

- Permanentes Handbuch der Postfreimarkenkunde mit Lichtdrucktafeln usw. Zweiter Teil: Ausland *. Leipzig: Hugo Krötzsch (s Co.) 1895-1908.

Bd 1. Bochmann, Eug. von: Die Postmar. ken des russischen Kaiserreiches. 1895. 178 S. u. 21 Taf.

Bd. 2. Djurling, H. \& R. Krasemann: Die Postmarken von Schweden 1855-1905. Nach amtl. Quellen hrsg. von Sveriges Filatelist-Förening. 1908. 116 S. m. Abb.

- Hannover. s. Krötzsch, Hugo: Permanentes Handbuch der Postfreimarkenkunde. Bd. 8.

- Ill. ausführl. Katalog über deutsche Postfreimarken mit Bekanntgabe der Auflagemengen, Restbestände, Lrkennungsmerkmale der Originale u. Neudrucke, sowie allen sonstigen dem Sammler wichtigen Anmerkungen aut Grundlage des anerkannt vorzügl. permanent. Handb. der Postfreimarkenkunde. Mit beigesetzten Preisnotierungen aus „Händlerkatalogen“ nebst Angabe, wie diese Preise zu den tatsächlichen Verkaufspreisen stehen u. welche Markenarten besondere Bewertung haben. Leipzig: Krötzsch. 1896. X, 150 S. m. Abb. $8^{0}$.

- Karte der deutschen Poststraßen in Frankreich 1870/71. Lichtdruck. Leipzig: Hugo Krötzsch. 1895. $4^{0}$.

[8. a. Krötzzch, Hugo: Permanenteg Handboch der Postfreimarkenkunde. Bid. 11 (als Beilage).]

- Lübeck. 8. Krötzsch, Hugo: Permanentes Handbuch der Postfreimarkenkunde. Bd. 9.

- Mecklenburg.Schwerin u. Mecklenburg-Strelitz. s. Krötzsch, Hugo: Permanentes Handbuch der Postfreimarkeukunde. Bd. 10.

- Norddeutscher Postbezirk. s. Krötzsch, Hugo: Permanentes Handbuch der Postfreimarken. kunde. Bd. 11.

- Die Postfreimarken des beiderstädtischen Postamtes Bergedorf. 2. Aufl. Leipzig: Hugo Krötzsch 1896. 169 S. u. 9 Taf. 8º

(Krötzsch, Handb. Bd. 4.)

- Die Prüfung ron Postwertzeichen und deren Entwerthungen. Vortrag. 8. Deutscher Philatelistentag; in: Stenograph. Bericht ü. die Verhandlgn. des 9. Deutschen Philatelistentages in Hamburg 1897.

- Statistik über Stückzahl und Wert meiner Postwertzeichen-Sammiung. OrdnungsgemäBer Übersichtsplan über die eigne Sammlung, unentbehrliche Unterlage für Versicherungsabschlüsse gegen Feuer und Einbruch, be- 
sonders bei entstebenden Frsatzansprüchen, ein praktischer Ratgeber für NachlaB-Erben. 2. verm. u. verb. Aufl. Iseipzig: Hugo Krötzsch it Co. (1910.) $76 \mathrm{~S}$. Leinen geb. $8^{\circ}$.

Krötzseh, Ilugo: Statistik über Stückzahl ... 3. verm. u. verb. Aufl. Ebda. [1916.] 80 S. Leinen geb. $8^{0}$.

(Die 1. Aufl. s. u. d. T. "Statietik".)

- s. a. Rommel, Otto \& Hugo Krötzsch: Braunschweig. leipzig 1893.

Kuhlmann, Karl, J.: Deutschland und Kolo. nien (Marken u. Ganzsachen) Spezial-Katalog Nr. 1. Hamnover: Selbstverl. [1901.] 23 S. $8^{\circ}$.

Kimucl, Julius: Katalog aller bekannten seit 1840 emittirten Briefmarken. Hrsg. unter freundlicher Mitwirkung der bedeutendsten Sammlerautoritïten Deutschlands von J. K. in Firma: Literarisches Museum. 12. u. 13. Aufl. Leipzig: Literarisches Museum. 1869. IV, 116 S. u. 1 Taf. m. 6 Markenabb. $8^{\circ}$.

[Bildet die Fortsetz, von Bauschke, Gustav: Kata $\log$ aller bekannten Briefmarken. Leipzig 1867.]

- Nachtrag. Ebda. 1869. 4 S. $8^{\circ}$.

_ 14. Aufl. Ebda. 1870. IV, 116 S. u. 1 Taf. m. 6 Markenabb. $8^{\circ}$.

Kinast, Walther: Preis-Verzeichniss der Postwerthzeichenhandlung von W. K. Berlin [1885.] 16 S. $8^{\circ}\left(16^{\circ}\right)$.

- Internationale Postwerthzeichen-Handlung. W. K. ... Preis-Verzeichniss. Berlin [1886.] 19 S. $8^{\circ}$.

_- [Neue Ausg.] Berlin [1887.] 26 s. $8^{\circ}$. - Nene Ausg.] Juli 1888. Berlin 1888. 24 S. $8^{\circ}$.

_- [Neue Ausg.] April 1889. Berlin 1889. 24 S. $8^{\circ}$.

- - [Neue Ausg.] Februar 1890. Berlin 1890. 28 S. $8^{\circ}$.

- - 25. Jubilänms - Preis - Verzeicbniss 1866-1891. Berlin 1891. 45 S. $8^{\circ}$.

- Nr. 26. Preis-Verzeichniss der Internationalen Postwertzeichen-Handlung von W. $K$. Mai 1893. Berlin 1893. 57 S. $8^{\circ}$.

- Nr. 27. Preis-Buch der Marken von Europa ...von W. K. ... Dezember 1894. 42 S. $8^{\circ}$.

[s. a. "Glasewald, A. E." u. "Schultze, Joh Otto" unter dem gleichnamigen Titel gedruckt.]

- Nr. 28. Preis-Verzeichniss der Internatio nalen Postwerthzeichen-Handlung von W. K. Oktober 1895. Berlin 1895. 70 S. $8^{\circ}$. [Fortsetzung ?]

Kunst, Die, im Bilde der Briefmarken. s. Vicenz, Ernst. (Vortrag, geh. auf dem XXII. Deutschen Philatelistentag in Kiel 1910.)

Kurpfalz. GroBe, R.: Das Postwesen in der Kurpfalz im 17. und 18. Jahrhundert. 'Tübingen 1902 .

Kütmann's Posthandbuch. [Früher u. d. 'T. "Der kleine Stephan", Posthandbuch.] Ein unentbehrl. Hilfsbuch für das Publikum. Ein tägl. Ratgeber für jeden Geschäftsmann. Handhuch für den gesamten In-u. Auslands-
Verkehr, nebst einem Verzeichnis der Nach. barpostorte, der wichtigsten Bestimmungen über den Telegramm- u. Fernsprechverkehr, einem postal. Straßenverzeichnis von Berlin u. enner Beilage: 'Tabelle der Gebührensätze. 19. Aufl. (abgeschlossen Januar 1913). Nach den amtl. Veröffentlichungen der Reichspostverwaltung bearb. von Fritz Vilich. Ausg. 1913/14. Uresden: Gerhard Kühtmann (1913.) XX, 287 S. m. 1 Tab. $8^{\circ}$.

Kiihtmaun's Posthandbuch. 20. Aufl. Kriegsausg. Bearb, von Oberpostassistent Otto Rehfeld. Die neuen Gebührensätze im Post- v. Telegraphenverkehr des ln- u. Auslands, insbesondere Österreich-Ungarns, Belgiens und Polens nach dem Stande vom 15. Nov. 1916. Euda. (1916). 68 S. $8^{\circ}$.

[8. a u. d. T. : ${ }_{n}$ Der kleine Stepban“.]

Kutscha, Franz: Die Hellasaufdrucke auf den Marken von Kreta, deren Fälschungen und Fälscher: Vortrag. s. Deutscher Philatelistentag; in: Stenograph. Bericht des XXI. Deutschen Philatelisten-Tages ... zu Kíarlsbad 1909.

Laband, Paul: Die Wertmarken. Sonderabdruck aus der Festschrift für Georg Cohn. (Abt. III: Die Postwertzeichen.) Zürich: Art. Institut Orell Füssli. 1915. 34 S. 8*.

Labuan. Heitmann, Ernst: Labuan im ,Großen Handbuch der Philatelie", Bd. 2, S. 363-367.

Lagos. Hoffmann, E.: Lagos im ,GroBen Handbuch der Philatelie", Bd. 2, S. 369-371.

Iarisch, Anselu: Engros-Preis-Liste. Wien: A. Larisch. $1885.32 \mathrm{~S}, 8^{\circ}$.

- Nummern-Liste zum Preis-Verzeichnis von A. L.; zu O. Koeppe's Permanentalbum u. zu Ferd. Meyer's Handbuch für PostmarkenSammler. München: A. Larisch. 1892. 40 S. $8^{\circ}\left(16^{\circ}\right)$.

- Postkarten-Katalog, enthaltend sämtliche bis 1890 erschienenen Postkarten. München: A. Larisch. 1891. VI1, 223 S. $8^{\circ}$.

- Prois-Verzeichniss sämmtlicher bjs 1886 erschienenen Postmarken, zugleich Manco-Liste zu Koeppe's Permanent-Album u. zu Ferd. Meyer's Katalog. Zsgest. von A. L. Wien: A. Larisch. 1887. 204 S. $8^{\circ}$.

_ - Zweite Aufl. München: A. Larisch, 1888. 188 S. $8^{\circ}$.

- Dritte Aufl. Ebda. 1890. 198 S. $8^{\circ}$.

- Vierte Aufl. Ebda. 1891. 220 S. $8^{\circ}$.

- Fünfte Aufl. Ebda. 1892. VI, 232 S. $8^{\circ}$. - - Sechste Aufl. Ebda. 1893. V1, 282 S. $8^{\circ}$.

Larsson, Jolın: Preisliste (ohne Verbindung) inber Skandinaviscbe Postwerthzeichen, zu beziehen vou J. L. Göteborg (Schweden): John Larsson. 1891. 10 S. $8^{\circ}$.

Lauber, August: Katalog aller bekannten seit 1830 bis Mitte 1866 angegebenen Briefmarken. Hrsg. von A. L. mit den Verkaufspreisen der Handlung von Hermann Goez in Stuttgart. Zweiter Abdruck. Stuttgart: F. Fibner. 1866. IV, 83 S. $8^{\circ}$. 
Laue, Robert: Geschichte des Vereins (Philatelia) s. "Philatelia" (Vereiu) in: Vereinsmittheilung der "Philatelia". Sondershansen 1887.

- Vereinsmitteilung der Philatelia. Vivat Philatelia. Festschrift zur Feier des dritten Stiftungsfestes. Sondershausen: Selbstverl. 1887. 24 S. $4^{\circ}$.

[Autographiert.]

Lederer, Paul: s. Lücke's Atlas der Briefmarken-Geographie.

- s. a. Opitz, C. \& Paul Lederer: Lücke's Länder- u. Weltverkehrskarte für Postwertzeichensammler.

Leede, 0sear: Das Sammeln von Postwerthzeichen nach Abstempelungen. s. Deutscher Philatelistentag, in: Stenograph. Protokoll über die Sitzung des XI. Deutschen Philatelistentages am 23. Juli 1899.

Leeward-Inselu. s. Antigua im Nachtr.

Lehmann, H.: Die einzig richtigen Adressen der hauptsächlichsten Brietmarkenhändler. Berlin: Selbstverlag. 1872. $8^{\circ}$.

Ausg. 1 mit 50 Adressen.

$$
\text { " } 2 \text { " } 100
$$

- Adressbuch der hauptsächlichsten Briefmarkenhändler [der ganzen Welt], enthaltend 625 Adressen. Berlin: Selbstverlag. 1873. (Druck: C. Ihring, Berlin, Dresdnerstr. 84.) 23 S. $8^{\circ}$.

[Bildet die 3. Aufl, vom vorhergebenden Werk des gleichen Verfassers.]

Leipzig. (lnternationale Ausstellung f. Buchgewerbe u. Graphik 1914.) Rübsam, Josef \& Rudolf Freytag: Postgeschichtl. Dokumente des Fürstl. Thurn u. Taxisschen Zentralarchivs zu Regensburg $\langle 1504-1909\rangle$. Regensburg 1914.

Leitfaden der Briefmarkenkunde (Philatelie). 8. Joessel, Alfons; Schidlof, Berthold; Senf, Gebrüder; Suppantschitsch, Victor.

Leitsïtze zur Bewertung der Abstempelungen. 8. Pirl, Paul.

Leman, Ed. v.: Spezialkatalog der Postwerthzeichen der Schweiz. Bearb. von E. v. L. Lausanne: Hans Kirchhofer. 1889. $31 \mathrm{~S} .8^{\circ}$.

- - Genf: Champion \& Cie. [1894]. 32 S. m. Abb. $8^{\circ}$.

Lengyel, S.: Die Wasserzeichen der ungarischen Postwerthzeichen. (Sonderabdr. aus d. Ill. Briefmarken-Zeitung v. 1. Sept, 1890.) Leipzig: Heitmann. 1890. 3 S. m. Abb. $4^{\circ}$.

Leopold, Ernst: E. L's Hand-u. Hilfsbüchlein für den Tauschverkehr. 1.-5. Taus. Bremen: August Marbes. 1893.38 S. $8^{\circ}$.

Liberia. Hoffmann, E.: Liberia im ${ }_{n}$ GroBen Handbuch der Philatelie", Bd. 2. S. 373-379.

Lieehtenstein. s. Caspart, Rudolf. (Vortrag geh. a. d. XXIV. Deutschen Philatelisten-Tag zu Marktredwitz 1912.)
Lienlıard, (Pfarrer): Die Entwerthungsarton der schweizerischen Briefmarken $1843-1863$, nebst sonstigen, damals auf den Briefen gebräuchlichen Aufdrucken. Zürich: Selbstverl. 1896. 14 S. u. 14 Taf. m. 283 Abb. $8^{\circ}$.

Lietzow, l'aul: Das schwarze Buch der Philatelie oder Neudruck und Fälschung von Postmarken und Briefumschlägen. Berlin: Selbstverlag. 1879. 72 S. $8^{\circ}$.

- Handbuch der Filatelie. T. 1. Vollständiges Verzeichniss aller amtlich ausgegebenen Postmarken u. deren genaue Beschreibung unter Berücksichtigung aller ihrer Verschiedenheiten (Farben, Sinnbilder, Wasserzeichen, Zähnung, Umrandung) mit beigesetzten Verkaufspreisen. Berlin: Selbstverlag. [Behr's Buchhandlung in Komm.] 1880. 113 S. $8^{\circ}$.

- Zweite bedeutend veränderte Aufl. Ebda. 1888. 127 S. $8^{\circ}$.

- - T. 2. Vollständiges Verzeichniss aller amtlich ausgegebenen Post-Briefumschläge, Streifbänder, Postkarten, Postanweisungen, Postscheine usw. und deren genaue Beschreibung unter Berücksichtigung aller ihrer Verschiedenheiten mit beigesetzten Verkaufspreisen. Fibda. 1882. 135 S. $8^{\circ}$.

- - 1'. 3. Vollständiges Verzeichniss aller seit Oktober 1879 ausgegebenen Postmarken und deren genaue Beschreibung unter Berücksichtigung aller ihrer Verschiedenheiten mit beigesetzten Verkaufspreisen. Ebda. 1885. $100^{\circ}$ S. $8^{\circ}$

— - T. 4. Vollständiges Verzeichnis aller seit Oktober 1881 ausgegebenen Briefumschläge, Streifbänder, Postkarten usw. und deren genaue Beschreibung. Ebda. 1886. 104 S. $8^{\circ}$.

- Seltenheiten und deren Preise. Preisverzeichniss der seltensten Postmarken aller Länder. Aufgestellt ron P. L. Berlin: Selbstverlag. 1884. 29 S. $8^{\circ}$.

- - Zweite veränderte Aufl. Berlin: Selbstverlag [u. Leipzig: Gebrüder Senf in Kowm.]. 1887. 56 S. $8^{\circ}$.

- Dritte veränderte Aufl. Ebda. 1890. 43 S. $8^{\circ}$.

- Spanien. Post- u. Verkehrswesen auf der pyrenäischen Halbinsel. (Beilage der "Deutschen Briefmarken-Zeitung" Oktober 1891.) Berlin: Dr. H. Brendicke. 1891. 8 S. $8^{\circ}$.

- s. a. Heitmann, Ernst: H's illustr. Handbuch für Briefmarkensammler usw.

Lima. Socièté Philatélique Sud-Américaine in Lima. s. Gutmensch, J.: Peru [Katalog].

Lindan, Joseph: Katalog zur Sammlung der Postwerthzeichen "Altdeutsche Staten sowie Deutsches Reich mit Kolonien" in ungebrauchten Stücken, bearb. vom Eigentümer J. L. in Heidelberg. Expesition Philatélique Internationale, Paris 1900. Classe 11I, Division I, Section B. Heidelberg: Selbstrerlag. 1900. II, $36 \mathrm{~S} .8^{\circ}$. 
Liudenberg, Carl: Aus vergangenen Tagen. Fine philatelistische Autobiographie von C. L. Zur Feier des fünfzigjährigen PhilatelistenJubiläums des Verfassers abgedruckt aus den Vereinsmitteilungen des Berliner Philatelisten-Klubs 1889. (Sonderdr.) Berlin: Selbstverlag des $\mathrm{B} . \mathrm{Ph} \cdot-\mathrm{Kl}$. 1912. 8 S. $8^{\circ}$.

- Die Briefbogen von Britisch-Ostindien. s. Berlin (Berliner Philatelisten-Klub) in: Festschrift z. Feier des zehnjähr. Bestehens des B. Ph.-Kl. Berlin 1898.

- Die Briefmarken von Baden. (Sonderdruck aus der "Deutschen Briefmarken-Zeitung" 1893.) Berlin: Dr. H. Brendicke. 1893. 68 S. u. 1 Lichtdr.-Taf. $8^{0}$.

- Lie Briefmarken von Baden, unter Benutzung amtlicher Quellen bearbeitet von C. L. Ebda. 1894. 172 S. u. 1 Lichtdr.'Taf. $8^{0}$.

- Die Briefumschläge der deutschen Staaten unter Benutzung amtlicher Quellen bearb. von C. L. H. $1-15$ in 3 Bdn. Berlin: Dr. H. Brendicke. $1892-1895.8^{\circ}$.

Bd. 1. H. 1. Braunschweig. 1892. $47 \mathrm{~S}$. m. Abb.

2. Mecklenburg-Sehwerin und Mecklenburg-Strelitz. 1892. $71 \mathrm{~S} . \mathrm{m} . \mathrm{Abb}$.

3. Lübeck. 1892. 32 S. m. Abb.

4. Thurn und Taxis. 1892. VIl, 72 S. m. Abb.

, 5/6. Norddeutscher Postbezirk. 1893. 120 S. m. Abb.

Bd. II. H. 7. Oldenburg. 1893. 39 S. m. Abb.

8. Baden. 1894. 68 S. m. Abb.

9. Hamburg und Bremen. 1894. 82 S. m. Abb.

10. Sachsen. 1894. 107 S. m. Abb. 11/12. Hannover. 1895. 176 S. m. Abb.

Bd. 111. H. 13. Bayern, 1895. 77 S. m. Abb. , 14/15. Württemberg. 1895. 269 S. m. Abb.

[H. 16|17. Preußen u, H. 18. Deutschland sind nicht erschienen.]

- Katalog der Marken-Sammlung des Reichs. Postmuseums. Bearbeitet von C. L. Berlin: Reichspostmuseum. 1888. VII1, 344 S. $8^{\circ}$.

- Die Postwerthzeichen Helgolands. Vortrag. s. Deutscher Philatelistentag; in: Stenograph. Bericht ü. d. Verbandlungen des 6. Deutschen Philatelistentages in Kiel 1894.

- Über die Postwertzeichen-Sammlungen des Reichs-Postmuseums. Vortrag. 8. Deutscher Philatelistentag; in: Stenograph. Bericht äber den 5. Deutachen Philatelisten-Tag in Berlin 1893.

- s. a. Berlin (Berliner Pbilatelisten-Klub). Festsebrift zur Feier des zehnjährigen Bestehens des B. Ph.-Kl.

- 8. a. Kalckhoff, Franz: Illustr. Verzeichnis aller bekannten Neudrucke.
Lindenberg, Carl: s. a. Teltz, O.: Grosses Handbuch der Philatelie. [Lfg.4-20] 1887[-90].

Lipski, Oberpostdirektions-Sekretär s. Stephan, Der kleine.

Ijiste, Die schwarze, des Vertraul. Korrespondenz-Blattes philatelistischer Vereine. Znsammenstellung der Warnungen aus den Jahren 1891-1899. Bearb. v. Hans Müller, Hannover. (Sonderbeil. zu Nr. 4 des Vertraul. Korresp.-Blattes v.10.April 1900.) (Hannover: Briefmarken Club 1900.) $20 \mathrm{~S} .8^{\circ}$.

Listen, Die sehwarzen, Kataloge u. Bücher auf dem Gebiete der Philatelio im Lichte des Rechts. Vortrag gebalten auf dem XV. Deutschen Philatelisten-Tag ... in P'forzheim 1903. s. Pauli, Emil.

Literarisches Mnseum in Leipzig: Katalog über alle bekannten seit 1840 bis Januar 1864 ausgegebenen Briefmarken, die zu den beigefïgten Preisen durch das Literarische Mluseum in Leipzig bezogen werden können. Leipzig: Literarisches Museum 1864. $23 \mathrm{~S}$. $8^{0}\left(32^{\circ}\right)$.

- - bis April 1864 ... Dritte Aufl. Ebda. 1864. 27 S. $8^{\circ}\left(32^{\circ}\right)$.

[8. a. Danneufelsen, W. F. u. Mana, Chrigtian unter gleichlautendem Titel gedr.]

- Katalog aller bekannten seit 1840 bis 1868 emittirten Briefmarken. Hrsg. unter freundl. Mitwirkung der bedeutendsten Sammlerautoritäten Deutschlands. Neunte Aufl. Leipzig: Literarisches Museum. 1868. IV, $111 \mathrm{~S} .8^{\circ}$.

- Zehnte Aufl. Ebda. 1868. 1V, 111 S. $8^{\circ}$.

- Elfte Aufl. Ebda. 1868. IV, 111 S. $8^{\circ}$.

- s. a Bauschke, Gustav: Katalog. Ausg. 1865 u. 1867 .

- s. a. Briefmarkenhandlung des Literarischen Museums in Leipzig.

— s. a. Kümmel, Julius: Katalog. Ausg. 1869 u. 1870 .

Literatur, philatelistische. Gerdt, A.: Herrn Schwefelmeier's zeitgemässe Betrachtungen über die Farbenbezeichnungen in der philatelistischen Litteratur. Leipzig 1891.

- Gottschalk, Albert: Die deutsche philatelistische Literatur 1894 bis 1897. Berlin 1898.

- König, Ludwig: Über philatelistische Literatur 〈mit Vorlagen〉. Vortrag gehalten auf dem XV1I. Deutschen Philatelistentag in Regensburg 1905.

- Moschkan, A.: Photographisches Souvenir für Deutscblands Philatelisten. Bl. 6. Philatelistische Literatur u. deren Förderer. Nos. sen 1875 .

- s. a. Bibliographien philatelistischer Litelatur.

Lobenstein (Reuss. j. L.). Katalog der Post. werthzeichen-Austellung in Lobenstein am 17. April 1892. Veranstaltet vom Vogtländischen Philatelisten-Verband, Vorort Zeulenroda. Lobenstein (Reuss. j. L.): [Ausstellungskomitee] 1892. 8 S. $8^{\circ}$. [Hektographiert.] 
Locher, Eduard: Spezial-Katalog sämtlicher Rotkreuz. u. Kriegsmarken 1914/16. Zürich, Bahnbofstr. 76: Edouard Locher 1916. $32 \mathrm{~S}$. m. Abb. $8^{\circ}$.

Lombarlei-Venetien. Kropf, H.: Wie Abstempelungeu der Marken von ÖsterreichUngarn u. Lombardei-Venetien 1850-1858. Prag 1899.

Jongueville, Frau von, Die Erfinderin der Postwertzeichen; in: Moschkau, A., Zur Geschichte der Philatelie.

Lossau, Julius: Hamburg (seine Post u. seine Postwertzeichen). s. Deutscher Philatelistentag; in: Stenograph. Bericht ü. die Verhandlgn. des 9. Deutschen Philatelistentages in Hamburg 1897.

Iöweulagen, Rudolf: Die Marken von Mexiko der Ausgaben 1895 bis 1899. (Sonderabdr. aus "Germania-Berichte" rom 30 . Septhr. 1900.) Blankenese, Hamburg: Selbstverlag. 1900. Einblattdr. $2^{\circ}$ (fol.).

lïwinger, Max H. s. Krehn, Julius: Internationales Philatelisten-Adressbuch.

Lübeck. Krötzsch, Hugo: Lübeck. (Krötzsch Handb. Bu. 9.) Leipzig 1893.

- Lindenberg, Carl: Die Briefumschläge von Lübeck. Berlin 1892.

- Lübeck im ,Grossen Handbuch der Pail-

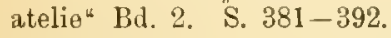

- Wilde, H.: Lübecks Postwertzeichen. Leipzig 1889.

- (Verein für Freunde der Briefmarkenkunde zu Lübeck.). Mitteilungen des Vereins ... s. Abt. "Zeitschriften".

Liibkert, Hugo: Handbuch aller bis 1881 bekanntgewoldenen Postwerthzeichen derRuralPosten von Russland. Wien: Sigmund Friedl. 1882. 132 S. m. Abh. $8^{\circ}$.

Lublin, (1.: Katalog für Briefmarken-Sammler. Berlin: [Selbstverlag. 1882-1894] $8^{\circ}$.

Nr. 1-6. nicht ermittelt.
7. [1882.] $32 \mathrm{~S}$.
8. $[1883] .32 \mathrm{~S}$.
9. [1883.] $36 \mathrm{~S}$.
10. nicht ermittelt.
11. [1885.] $40 \mathrm{~S}$.
12. [1886.] $46 \mathrm{~S}$.
13. [1886.] $92 \mathrm{~S}$
14. 1887. $138 \mathrm{~S}$.
15. [1888.] $144 \mathrm{~S}$.
16. [1890.] $106 \mathrm{~S}$.
17. [1891.] $112 \mathrm{~S}$.
18. [1892.] $120 \mathrm{~S}$.
19. [1894.] $34 \mathrm{~S}$.

- Separat-Abdruck der Deutschen Staaten aus (t. Lublin's Illustr: Katalog Nr. 18. Berlin: [Selbstrerlag. 1892.] $16 \mathrm{~S} .8^{\circ}$.

Liicke, C. F.: Lücke's Atlas der BriefmarkenGeographie. 198 Karten aller Staaten, die jemals Marken rerausgabten. Bearb. von C. Opitz \& P. Lederer. Vollständig in $2 \mathrm{Lfg}$.

Lfg. 1. Europa-Asien. 90 Karten in fünffarbigem Stich.

2. Afrika-Australien. 108 Karten. [lm Erscheinen.]

Leipzig: C. F. Lücke, G.m. b. H. 1917. $8^{\circ}$.

Liieke, C. F.: Fehlliste zum Schaubek-Albun. Übersichtsbuch zur schriftl. Festlegung des Bestandes einer jeden Briefmarkensammlung u. zur Ausübung einer ständigen Kontrolle über dieselbe. 4. Aufl. Leipzig: C. F. Lücke o. J. 123 S. +4 S. Anh. f. Notizen. 80.

- Jahrbuch für Briefmarken-Sammler 1907 bis 1908. Allen Briefmarken-Sammlern gewidmet vom Verlag des Schaubek-Album. Leipzig: C. F. Lücke, 1907. 64 S. $8^{0}$.

- - - 1908-1909. Ebda. 1908. 64 S. $8^{\circ}$.

- - - 1909-1910. Mit Kalendarium, geographisch-statistischen Notizen $u$. Tabellon zur Aufnahme des Bestandes von BriefmarkenSammlungen u. s. w. Ebda. 1909. 64 S. $8^{\circ}$.

- - - 1910-1911. Mit Kalendarium, Münztabellen, sowie Rubriken zur Hintragung des Bestandes von Briefmarken-Sammlungen. Utberreicht vom Verlag des Schaubek-Albums. Ebda. 1910. 64 S. $8^{\circ}$.

- - 1911-1912. - Ebda. 1911.64 S. $8^{0}$.

- - Inventur- u. Jahrbuch für BriefmarkenSammler 1912-1913. Mit Kalendarium, Münztabellen, sowie Rubriken zur Eintragung des Bestandes von Briefruarken-Sammlungen. Leipzig: C. F. Lücke. 1912. 64 S. $8^{\circ}$.

- - - 1913. Mit Kalendarium 1913/14 u. s. W. Ebda. 1913. 64 S. $8^{\circ}$.

- - lnventurbuch für Briefmarken-Sammler 1914. Uberreicht vom Verlag des SchaubekAlbums. Leipzig: C. F. Lücke. 1914. 64 S. 80.

- - Preisverzeichuis ïber Schaubeks illu. strierte Briefmarken-Albums sowie über sonstige Bedarfsartikel für Briefmarkın Sammler. [Ausg.] 1904. Leipzig: C. F. Lücke. [1903.] 48 S. $8^{\circ}$.

- - - [Ausg.] 1905. Ebda. [1904.] 48 S. $8^{\circ}$.

[Fortsetzung unter "Lücke's Ratgeber".]

_- Ratgeber 1906. Allen Postwertzeichen. Sammlern gewidmet vom Verlag des Schauhek-Album. Leipzig: C. F. Lücke. 1906. 80 S. $8^{\circ}$.

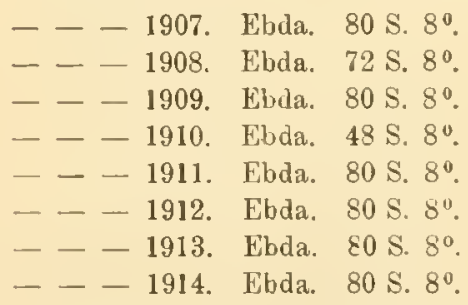

- s. a. Opitz, C.: Lücke's Länder- u. Weltverkehrskarte für Postwertzeichen Sammler. 
Luftpostmarken. Hobrecker, $K_{\text {. }}$. Die ersten deutschen Luftpostmarken. Berlin 1912.

Lutler, A. F.: Briefmarken-Preiscourant. Braunschweig: Selbstverlag. 1869. $16 \mathrm{~S} .8^{\circ}$.

Luxemburg. Faber-Lakaff: Timbres-posteBriefmarken. Prixcourant de gros et de détail de la maison F.-L. (Timbres-postede Luxembourg.) Luxembourg: [Selbstverlag] 1897.

[Mit teilweise deutechem Text.]

- Kobl, Paul: 1llustr. Katalog der Freimarken von Europa. Abt. 2 "Luxemburg".

- Lindenberg, Carl \& Emil Hoffmann: Luxemburg im "GroBen Handbuch der Philatelie*, Bd. 2. S. $395-450$.

- Sauer, C. Cl.: Permanonter Katalog. „Luxemburg."

Nacao. N. N.: Macao im "Groben Handbuch der Philatelie", Bd. 2. S. $451-460$.

Madagaskar (britische Konsularpost). Heitmann, Ernst: Madagaskar im ,GroBen Handbuch der Philatelie", Bd. 2, S. 465-476.

Madeiru. F. M.: Madeira in „Großen Handbuch der Pbilatelie", Bd. 2. S. 477-482.

Magdeburg. (Verein für Briefmarkenkunde zu Magdeburg.) Die Internationale Ausstellung offizieller Postwertzeichen, veranstaltet zur 50 jähr. Jubelfeier der Kinfúhrung der Briefmarke, zu Magdeburg in den Sälen der ${ }_{n}$ Freundschaft" vom 4.-II. Mai 1890. Magdeburg: Verein f. Briefmarkenkunde. 1890. 8 S. $4^{0}$.

- Führer dureh die Internationale Ausstellung officieller Postwertzeichen, veranstaltet zur 50 jähr. Jubelfeier der Briefmarke am 4. bis 11. Mai 1890 in den Sälen der Gesellschaft ${ }_{n}$ Zur Freundscbaft". Ebda. 1890. 18 S. $8^{\circ}$.

- Sammlung der Erkennungs-Zeichen seltener Briefmarken. Nr. 1. 9. Februar 1891. (Sonderabdr. aus ,1llustr. Brieimarken-Zeitung ${ }^{*}$ 1891.) Ebda. 1891. 4 S. $8^{\circ}$.

- s. a. Roggenstroh, H.: Die Postwertzeichen von Rumänien, u. Trillich, H.: Das Wasserzeichen und seine Erkennung in Postwertzeichen.

Mährisch-0stran, Osterreich. (Internationaler Philatelisten-Verband zu Mährisch-Ostrau.) s. Verbandsuachrichten des 1. Ph.-V. M.-O. Abt. "Zeitschriften".

Maier, M. Kurt: Kataloge der BriefmarkenVersteigerungen. s. Auktions-Kataloge und Briefmarken-Verkteigerungen.

- Preisliste 1917. Berlin W.8, Friedrichstr. 185. M. Kurt Maier 1917. 128 S. $8^{\circ}$.

[в. a. u. Ruben, Edvard M.: Briefmarkenpreisliste Nir. 15.]

Mainz. Mainzer Post s. Beck, Otto.

Malta. Heitmann, Ernst: Malta in GroBen Handbuch der Philatelie *, Bd. 2. S. 487-496.

- Kohl, Paul: 1llustr. Katalog der Freimarken von Europa. Abt. 3 "Malta*.
Manco-Listen (Fehllisten). s. Dauth, H. J.; Heitmann, Ernst; Larisch, A.; Lücke, C. F.; Oppenfeld, E. v.; Senf, Gebrüder.

Mangold, 0tto: Catalogue de l'Union Postale Universelle. Abdruck von den General-Post. ämtern überlassener Schriftstïcke. Hrsg. von H. J. Dautb. Amtliche liste: Portugal u. Colonien, bearb. u. m. Preisen verseben von O. M., Fraukfurt a./M. Frankfurt a./M.: H. J. Dauth [1891.]. 21 S. $8^{\circ}$.

- s. a. Dauth, H. J. Catalogue de l'Union Postale Universelle. Audruck postamtlicher Documente.

Mann, Christian: Adressbuch europäischer Markenbändler. Hrsg. u. bearb. von C. M. junior. Leipzig: Hermann Luppe in Komm. 1865. - S. $8^{\circ}$.

- Katalog über alle bekannten seit 1840 bis Mai 1864 ausgegebenen Briefmarken, die zu den beigefügten Preisen durch C. M. junior in Leipzig bezogen werden können. Dritte Aufl. Leipzig: Selbstverl. [u. Hermann Luppe in Komm.] 1864. 29 S. $8^{\circ}\left(32^{\circ}\right)$.

[8. a. Literarisches Museum in Leipzig unter gleichlautendem Titel gedr.j

- Katalog über alle bekannten seit 1840 bis August 1865 ausgegebenen Briefmarken $u$. 8. W. Ebda. 1865. 38 S. $8^{\circ}\left(32^{\circ}\right)$.

- Katalog ... bis August 1868 ... Ebda. 1868. 41 S. $8^{\circ}$.

- Katalog ... bis Dezemher 1870 . . Fibda. 1871. 42 S. $8^{\circ}$.

- Nacbtr. 1. Ebda. [1871.] 4 S. $8^{\circ}$.

- Katalog aller seit 1840 bis auf die neueste Zeit ausgegebenen Briefmarken zu den ver. zeichneten Preisen, welche durch C. M. junior bezogen werden kônnen. Ebda. 1876. 55 S. $8^{\circ}$.

Marbes, August: Philatelistisches Adressbuch. Verzeichnis der bedeutensten Briefmarkensammler u. -Händler der Welt. Zsgest. vom Verleger. Bremen: Marbes. 1890. 80 S. $8^{\circ}$.

- Zweite, bedeutend verm. Ausgabe. Ebda. 1896. $144 \mathrm{~S} .8^{\circ}$.

- s. a. Finke, Georg: Katechismus des Briefmarkensammlers, u. Leopold, Ernst: Handu. Hilfsbüchlein $f$. $d$. Tauschverkebr.

Marburg. Beitrag zur Geschicbte des Postamts M. 1784-1884 s. Ruhl, J.

Marcus, M. II.: Katalog u. Preisliste orientalischer Postwerthzeichen. Constantinopel: Selbstverl. 1896. 32 S. $8^{\circ}$.

Marken auf Brief. s. Neidhard, Vortrag geh. auf dem 5. Deutschen Philatelistentag in Berlin 1893.

Marken, geteilte. Utbersicht üher die in den Jahren 1840-1911 ausgegebenen u. verwendeten geteilten Marken, s. Genth, F.

Markenbogen. s. Schwaneberger, Hugo: Uber ganze Bogen. Vortrag, geh. auf dem XVI. Deutschen Philatelistentag in Leipzig (und Halle a. S.) 1904. 
Markenconservirung, Über. s. Kalekhoft', Franz. (Vortrag geh. auf den 6. Deutschen Philatelistentag in Kiel 1894.)

Markenhaus der Anstalten zu betlel bei Biclefeld. Briefmarken-Katalog vom M. der A. zu B. bei B. 1894-95. Betlel 1894. 110 S. $8^{\circ}$. $\left(16^{\circ}\right)$.

- - [Neue Ausg.] 1895 -96. Ebda. 1895. 147 S. $8^{\circ}$. $\left(16^{\circ}\right)$.

- - Neue Ausg.] 1896-97. Ebda. 1896. $144 \mathrm{~S} .8^{\circ},\left(16^{\circ}\right)$.

Marken- M. Manzsachenhaus (r. m. b. 11. Berlin. [hatalog der] Briefmarken-Versteigerungen v. 22.-24.Januar 1917. BerlinW. 8, Friedrichstr. 162. Marken- u. Ganzsachenhaus 1. m. b. H. 1917. 82 S. m. Markenabb. $4^{\circ}$.

- Die gegenwärtige Lage des Briefmarkenmarktes und der Einfluss unserer letzten Versteigerung auf dio Gestaltung der jetzt geltenden Preise fuir Raritäten. Vergleichende Uebersicht über die Preisgestaltung einiger beliebter Seltenheiten. Berlin W.8, Friedrichstr. 162: Narken-u.GanzsachenhausG.m.b.II. (1917). 7 S. 4\%.

Nebst Beil.: Ergebnis der Versteigerung vom 22.-24. Januar 1917. 8 Blatt gummiert u. perforiert. $4^{\circ}$.

- Die neue Preis-Grundlage 1917 bis 1918. Berlin W. 8, Friedrichstr. 162. Marken- u. Ganzsachenhaus G.m.b.H. 1917. 128 S. $8^{\circ}$.

[в. a. n. Ruben, Edvard, M.: Briefmarkenpreisliste Nr. 15.]

Markenmiiller. s. Knorr, Louis.

Markensammlers. Wegweiser f. Markensammlel Marokko. Anderech, Max: Die deutsche Post in der Türkei, in China und in Marokko, unter besonderer Berücksichtigung der anderen fremden Postanstalten in diesen 1,ïndern. Mit 3 Karten. Berlin: R, von Deckers Verlag 1912. $183 \mathrm{~S} .8^{\circ}$.

[Siebe auch unter "China u. Türkei.]

- Rommel, O.: Marocco, seine Post und seine Postwerthzeichen. Leipzig [1907].

- Kleinere Abarten bei den Marken fü. die fremdherrlichen Postanstalten in Marocco. Leipzig [1908].

Martiuctz, J. A.: Internationales Briefmarkenhändler-Adressbuch. International stamp dealer's directory. Hrsg. v. J. A. M., Arnold Fleischmann, Robert Odabassian. Wien: 1877. $1 \mathrm{~V}, 64 \mathrm{~S} .8^{\circ}$.

Martinique. Vittinghoff-Schell, Frhs. v.: Martinique in ,Grossen Handbuch der Philatelie". Bd. 2. S. $497-508$.

Maschinenstempel. Apstein, Dr.: Über Maschinenstem pel. s. Deutscher Philatelisten. tag; in: Die Vorträge vom XX1I. Deutschen Philatelistentage in Kiel. Leipzig 1910.

,Matador ${ }^{\text {. }}$ s. Freudenberg, Werner.

Mauritius. Vittinghoff-Schell, Frhr. von: Mauritius im Grossen Handbuch der Pliilatelie*. Bd. 2. S. 509-557.

Maus, J.: Die Post in China. Vortrag. s. Deutscher Philatelistentag; in: Stenograpl. Bericht ii. d. XI1. D. Ph. zu Berlin Iy0I.
Maus, J.: Die Schiffspost auf dem Bodensee. Vortrag. 8. Deutscher Philatelistentag; in: Stenograph. Protokoll iuber die Sitzung des Xl. Deutsclien Philatelistentages.

Meckleuburg-Sclwerin. Anheisser, J. H.: Mecklenburg-Schwerin (u. Strelitz) im n GroBen Handbuch der Philatelie“. Bd.2. S. 559-584.

- Couvert-Emission 18565 Shilling blau (Fälschung); in: Moschkau, A., Zur Geschichte der Pbilatelie.

- Krötzsch, Hugo: Mecklenburg-Schwerin. 1. Autl. Leipzig 1893.

Mecklenburg.Schwerin u. MecklenburgStrelitz. 2. Autl. Leipzig 1895.

(Krötzвch, Handb. Bd. 10.)

Lindenberg, Carl: Die Briefumschlïge von Mecklenburg-Schwerin u. Strelitz. Berlin 1892. Noeller, C.: Geschichte des Landes-Postwesens in Mecklenburg-Schwerin. Schworin i. M. 1897.

Mecklenburg-Strelitz, Anheisser, J. H.: Necklenburg-(Schwerin u.) Strelitzim Grossen Handbuch der Philatelie". Bd.2. S.559-584.

- Krötzsch, Hugo: Necklenburg-Schwerin u. Mecklenburg-Strelitz. 2. Aufl. Leipzig 1895. (Krötzsch, Handb. Bd. 10.)

Lindenberg, Carl: Die Briefumschläge von Necklenburg-Schwerin u.Strelitz. Berlin 1892.

Mehler, J. B.: Das fürstliche Haus Tliurn u. Taxis in Regensburg. Zum 150 jähr. ResidenzJubiläum. Regensburg: Habbel 1899. XV, 299 S. m. Abb. $8^{0}$.

(S. 265-268. Hartmann: Der BriefmarkensammlerVerein ${ }_{n}$ Ratisbona" Regensburg. Sondersammlgn. von Thurn u. Taxis.)

Melkeustein und Photiadès. 1888. Preislisle Orientalischer Briefmarken, Ganzsachen etc. Constantinopel: Selbstverl. 1888. IV, 28S. $8^{\circ}$.

Meuze, 0.: Adress-Verzeichniss der Briefmarken-Händler und Samm]er, [Hamburg: Selbstver]. 1876.]

[Autographiert.]

Mercure. Zur Geschichte der Österreichischen Mercure. s. Friedl, Sigmund.

IIerläuder, Ludwig: Die Administrationsmarken Apgyptens. Gratis-Ausgabe während der Ausstellung dieser Marken von G. Koch in Cairo (Aegypten). Zur Erinnerung an die 1. Internationale Postwerthzeichen-Ausstellung in Wien rom 20. April bis 4. Mai 1890 .. I Leipzig: Ernst Heitmann in Komm. 1890. 8 S. m. Abb. $8^{\circ}$.

[Zugleich Sonderdruck aus „Der Philatelist". Feluruar Nr. 1890 ]

Metzger, 0. 11.: Alfred Moschkau. Ein Lebensbild. Maria Enzersdorf (Nieder-Oesterreich): Selbstverlag 1915. (Mödling bei Wien. Franz Gschmeidler in Komm.) $48 \mathrm{~S} .8^{\circ}$.

Metzkes, Erust: Spezial-Sammlung der Marken des Deutschen Reiches, seiner Auslandposten u. Kolonien, Barmen, Frnst Netzkes [1912]. $18 \mathrm{~S} .8^{\circ}$.

Mexiko. Fouré, Georges: Nachrichten über Porto de Mar-Marken, Mexiko. Berlin 1881. 
Mexiko. Schell, Frhr, von, J. H. Anheisser u. A. Püschel: Mexico im Grossen Handbuch der Philatelie" Pd. 2. S. 585-972.

Ieyer, A.: Die deutsche Post im Weltpostverein ฉ. im Wechselverkehr. Frläuterungen zum Weltposthandbuch u. zum Handbuch für den Wechselverkelir. Nach dem Stande v. 15. VI. 1901 bearb. Berlin: Julius Springer 1901. VIIl, 337 S. $4^{\circ}$. (gl. 80$)$.

- - 2, verm. u. verïnderte Aufl, nach dem Stande vom 15. VIl. 1908 bearb. v. H. Herzog. Ebda. 1908. XIl, 416 S. $4^{0}$. (gr. $\left.8^{\circ}\right)$.

Meyer, Ferdiuaud: Afghanistan, seine Post und seine Postwertzeichen. Wien: Sigmund Friedl 1879. 23 S. m. Abb. $8^{\circ}$.

- Handbuch für Postmarkensammler für den permanenten Gebrauch bestimmt. Vollständiges Verzeichniss u. Beschreibung aller amtlich ausgegebenen Postmarken sowie sämmtlicher Privatmarken ... Nürnberg: Georg Zechmeyer 1881. XV, 656 S. $8^{\circ}$.

- Erster Naclitr. Ebda. 1881. IV, $78 \mathrm{~S} .8^{\circ}$.

- Z Zweiter.

1882. $1 \mathrm{~V}, 80 \mathrm{~S} .8^{\circ}$.

- Dritter $₫$ Von Ferd. Meyer. Revidiert von Dr. jur. P. Kloss. Ebda. 1883. VIII, 50 S. $8^{\circ}$.

- Vierter Nachtr. Zsgest. von Dr. jur. P. Kloss. Ebda. 1884. 90 S. $8^{\circ}$.

- - Fünfter Nachtr. Zsgest. von Dr. jur. P. Kloss. Ebda. 1886.87 S. $8^{\circ}$.

- Sechster Nachtr. Zsgest. von Dr. jur. P. Kloss. Ebda. 1888. $119 \mathrm{~S} .8^{\circ}$.

- Siebenter Nachtr. Zsgest. von Dr. jur. P. Kloss, nebst Register zu den Nachträgen 1-7. Ebda. 1890. 127 S. $8^{\circ}$.

- Katalog der Postwerthzeichen des ottomaniscben Kaiserthums. Wien: TSigmund Friedl]. 1878. $16 \mathrm{~S} .8^{\circ}$.

[Auch als Gratio-Prämie zum Jg.1877 der Wiener 11luotr. Briefmarken-Zeitung.]

- s. a. Antoniades, J. T.: 1892-1893. Katalog über Orientalische Postwertzeichen.

- s. a. Dauth, H. J.: Manco-Liste zu Ferd. Meyer's Katalog.

- s. a. Hruby, R.: Katalog über Orientalische Postwertlizeichen.

- 8. a. Larisch, A.: Preis-Verzeichniss sämmtl, bis 1886 erschienenen Postmarken nebst Nummernliste.

- s. a. Raditch \& Co., G.: Katalog über Orien. talische Postwerthzeichen.

Michalloff, Johanues: Preisliste über Marken, Briefumschläge, Karten etc. von Finland. Wiborg, Finland: Selbstverl. 1891. 10 S. $8^{\circ}$.

- Detail-Preisliste über finnische Brietmarken etc. Ebda. 1893. 1 S. $4^{0}$.

Michel, Ilugo: Europa-Katalog 1910. Apolda: Hugo Michel (1910), 114 S. 80.

[Eiuband rotbraun.]

- 1911. Ebda. (1911). 126 S. $8^{0}$. [Einband hellgrau.]
Michel, Il ugo: liuropa-líatalog 1912. Libda. (1911). $136 \mathrm{~S} .8^{\circ}$.

[Einband oliv.]

- - Nachtr. No. 1 April 1912. $16 \mathrm{~S}$.

No. 2 Juli $1912.16 \mathrm{~S}$.

No. 3 October 1912. $24 \mathrm{~S}$.

— 1913. Ebda. (1912). 144 S. $8^{\circ}$.

[Eiuband violett.]

- - Nachtr. Nr. 1 April 1913. $28 \mathrm{~S}$.

- $\quad$ Nr.2 Juli 1913. $16 \mathrm{~S}$.

Nr. 3 October 1913. $32 \mathrm{~S}$.

- 1914. Ebda. (1913). 157 S. $8^{\circ}$.

[Einband kornblau.]

- Nachtr. Nr. 1. April 1914. 24 S.

$\mathrm{Nr}$. 2. Juli 1914. $16 \mathrm{~S}$.

Nr. 3. Oktober 1914. $16 \mathrm{~S}$.

- - 1915. Kbda. (1914). 166 S. $8^{\circ}$.

[Einband feldgrau.]

- Nachtr. Nr. 1. April 1915. $32 \mathrm{~S}$

- _ Nr.2. Juli 1915. 16 S.

- $\quad$ Nr.3. Oktober 1915. $16 \mathrm{~S}$.

- - 1916. Weimar: Hugo Michel 1915.

174 S. $8^{\circ}$.

[Einband blutrot.]

- Nachtr. Nr. 1. April 1916. $20 \mathrm{~S}$.

- $-\quad \mathrm{Nr} .2$. Juli 1916. $24 \mathrm{~S}$.

- Nr.3. Oktober 19l6. $32 \mathrm{~S}$.

- 1917. Ebda. 1917. 186 S. 80.

[Hinbagd gelbbraun.]

- Michel's Mignon. Jan. - März 1914. Apolda: Hugo Michel 1914. 92 S. $8^{\circ}$.

- April-Juni 1914. 94 S. $8^{\circ}$.

- Juli-Septbr. 1914. 94 S. $8^{\circ}$.

- - Oct.-Dezbr. 1914. 96 S. $8^{\circ}$.

- Miclıel's Mignon. Jan. - März 1915. Apolda: Hugo Michel (1915). 94 S. $8^{\circ}$.

_ - April-Juni1915. Ebda.1915.94S. $8^{\circ}$.

_ - Juli-Septbr.1915. Ebda.1915.94S.80.

— - Oct.-Dezbr.1915. Ebda.1915.96S.8º.

- - Januar-März 1916. IVeimar: Hugo

Michel 1916. $95 \mathrm{~S} .8^{\circ}$.

_ - A pril-Juni 1916. Ebda. 1916. 96S. $8^{\circ}$.

— - Juli-Oct. 1916. Ebda. 1916. 98 S. $8^{\circ}$.

[Erecheint ab Oct. 1916 nicht mebr.]

Mielelseu, G.: Über die Postwerthzeichen der Vereinigten Staten von Columbien. Vortrag. s. Deutscher Philatelistentag; in: Stenograph. Bericht ü. die Verhandlgn. des 9. Deutschen Philatelistentags in Hamburg 1897.

Miguon-[Katalog] Europa. s. Michel, Hugo.

Miniaturbilder aus dem Gebiete der Wirtschaft s. Hermann, Emanuel: Die Correspondenz. karte.

Mirabaud, Paul \& A. de Reuterskiöld: Die Schweizerischen Postmarken, $1843-1862$. [Deutsche Übersetz. von "Les timbres - poste Suisse, $1843-1862$ der gleichen Verf. in 150 numerierten Exemplaren gedr.] Paris 1899 [1900]. XIV, 280 S. u. 14 Taf. $4^{\circ}$.

Misson, Estrelle: Geschichte der telephonischen Marken von Belgien. Vortrag mit Lichtbildern von Fräulein Misson, geh. auf dem XVIII. Deutschen Philatelistentag in Nüın- 
berg. s. Deutscher Philatelistentag; Stenograph. Bericht ü. d. IVIII. D. Ph.... in Nüınberg 1906.

Missstïude in del Philatelie s. Motz, Hermann.

Mitlelmann, Ilermann: Adressbuch der überseeisclien und der orientalischen Briefmarkensammler. Lahr (Baden): Mloritz Schauenburg 1891. $53 \mathrm{~S} .8^{\circ}$.

- Katalog der existierenden Briefmarken7eitungen. Hrsg. v. H. M. Mähr.-Ostrau : Julius Litt] 1889. 32 S. 8".

Mocambique. Vittinghoff-Schell, Frhr. von: Mozambique; im Grossen Handbuch der 1'hilatelie" Bd. 2. S. 1047-1063.

Modena. Diena, E.: Modena im Grossen Handbuch der Philatelie" Bd. 2. S.973-995.

Molda॥ s. Rumänien.

Moeller, C.: Geschichte des Landes-Postwesens in Mecklenburg-Schwerin. Schwerin i. Mecklbg.: Bärensprung 1897. 359 S. m Abb. $8^{0}$.

Monaco. Kohl, Paul: Illustr. Katalog der Freimarken von Europa. Abt. 7: ,Monaco".

- Vittinghoff-Schell, Frhr. von: Monaco; im "Grossen Handbuch der Philatelie" Bd. 2. S. $997-1012$.

Ioens, J. B.: Nieder]ändische Essais. s. Berlin (Berliner Philatelisten-Klub) in: Eestschrift z. Feier des zehnjähr. Bestehens des B. Ph.-Kl. Berlin 1898.

- Die Postmarken von Württemberg. s, Berlin. (Deutscher Verein f. Philatelie in Berlin) in: Vorträge ïber Postwerthzeichen. Ber]in 1881.

Montenegro. Bickel, Otto: Montenegro im "Grossen Handbuch der Philatelie Bd.? S. $1013-1036$.

- Griebert, H.: Montenegro. Spezial-Album [und Katalog] für die Briefmarken Montenegros London (1913).

Montserrat. Vittinghoff-Sche]], Frhr. v.: Montserrat; im ,Grossen Handbucl der Plilatelie* Bd. 2. S. 1037-1046.

Mosambik s. Moçambique.

Mosehkau, Alfred: Biographie; in: Jeran, O.: Wegweiser für Verkehrswertzeichen-Sammler.

— - s. a. Metzger, O. H.; Nüssle, Dr.; Schmidt, Rudolf.

- Briefmarken-Zeitung für die Jugend. Für die Käufer unseres Briefmarken-Albums hrsg. von Dr. A. NI. Leipzig: Ernst Heitmann [1889.]

[Reklameschrifc gelegeotlich einer Neuaugy. von Schwanebergers Briefmarken-Album ]

- Erinnerungen an 1870-71. Photographien in Visitformat: Ballonbriefe, Taubenpostdepeschen, Postkarten aus der Belagerung von Paris wiedergebend. Nossen: Moschkau's photogr. art. Institut 1875.

[8.a. Moschkau, Alfred : Photogr. Souvenirf, Deutschlands Philatelistea. BL. I.]

- Geschichte der Briefmarken u. der Philatelie. s. Moschkau, Alfred: Die Wasserzeichen auf den... Briefmarken. „Dritte gänzl, umgearb. Aufl."
Moschkau, Alfred: Zur Geschichte Ifl Philatelie. Gesammelte Beiträge zur Geschichte der Briefmarken und der Briefmarkenkunde. Mit Portr. u. Illustrationen. Leipzig: Louis Senf 1879.80 S. $8^{\circ}$.

(Philatelistieche Bibliothek Bd.4.)

Iuhalt: Frau von Longugville, die Erfinderin der Postwertbzeichea. - Sir Rowlsnd Hill, Begríoder dee Posimarkenweeen8. - Dr. Heiuich Stephau, Generalpostdirektor des Dentscben Reichee. - Prinz Wilbeln von Preußen. Ein fürstlicher Plibatelist. - Fürotliche Plilistelisteu. - Wer sarumelt eigentlich Briefmarken? lieantwortung dieser Frage durch geine (Dr. Moschkau'e) Autographeneamming von Philatelisteu. Rükeriunerungen. - Die Briefmarken auf dor Wiener Weltausstellung. - Die Converte Sardiniene und ihre Nachahmungen. - Eine Curiosität von Braungchweig. - Die Connell-Marke" . Das Sammelu ganzer l'ouverte. - Das Sammela vou Stempelmsrken. - Pariser Commune-Markeo. - Amorikanisches postamtliche Papiergeld. - Chinesieche Couverte. - Die Lokalpostmarken Russlands. - Zum Filschungrwesen. Filschungen: Basler Täubchen, Nespel 1860 mit savoyigchem Kreuz, MecklenLurg-Schweriu CourertEmission 18565 Shilling blau, Philippinen I. Fmission, Rom 1 Scudo und 50 Bajochi, die Certificationes von Columbia ual ibre Nachahmungen, Sardinien \$ lire brouce und Finulsad-Couvert $181510 \mathrm{Kop}$, Bchwarz, Zürich-Filschungen, eine Schwindel-Emisgion von Grönland, Japan, Toecsna.Marken, dis 27 para der Moldau, Peru Medio Peeo, Spaniens Bär. - Auch eine Rückerinnerung.

- Handbuch für Essaissammler. Verzeichniss aller bis dato bekannten officipllen postalischen Essais. Leipzig: Louis Senf 1875. $51 \mathrm{~S}$. u. 1 Taf, m. Abb. $8^{\circ}$.

(Philatelistioche Bibliothek Bd. 2.)

- Handbuch für Postmarkeusammler. Kin Catalog aller von 1653-1876 emittierten Postmarken, Couverts, Karten, Streifbänder etc. etc., mit mehreren Hundert erläuternden Notizen, mehreren Tafeln llustrationen, dem Portrait und einer von Dr. Nïssle bearbeiteten Biographie des Verfassers. Mit beigedr. Verkaufspreisen der Briefmarkenhandlung von Louis Senf, Leipzig. 2. Aufl. Leipzig: Louis Senf 1876. 223 S. $8^{\circ}$.

(Philatelistigche Bibliotbek Bd 3.)

- Nachtrag aller seit Dezember 1875 bis 1. Juli 1876 emittirten Briefmarken, Couverts, Postkarten, Anweisungen, Streifländer. Ebda. 1876. $18 \mathrm{~S} .8^{0}$.

[1. Aufl. s, u. d. T.: Moschkau, Alfred: Katalog über alle oeit 1818 bis April 1874 ausgegebenen Briefmarken.]

- Handbuch ... 3. verm, u. verb. Aufl. Ebda. 1877. 268 S. + 89 S. m. Abb. $8^{\circ}$.

(Philatelietiache Bibliothek Bd.3.)

- - 4. verm. u. verb. Aufl. Ebda. 1880. 315 S. nebst 1 Portr. u. 99 S. m. Abb. $8^{\circ}$.

(Philatelistische Bibliothek Bd. 3.)

- - Nachtrag (zur 4. Aufl.). ... Mlit 54 Albb. Dresden: Ernst Petritz 1881. 38 S. + 6 S. m. Abb. 8*.

- - 5, von IV. Herrmann bearb. Aufl. Illustrierter Katalog aller seit 1653 bis Ende 1883 erschienenen Postmarken. Leipzig: Gebrüder Senf 1884. 512 S. u. 168 S. m. A bb. $8^{\circ}$.

(Philatelistische Bibliothek $\mathrm{Bd} .3$ )

- - Nachtr. [1.] bearb, von WV. Mermunn. Ebda. 1885. S.513-596+169-182 u.Abb. $8^{\circ}$. 
Moschkau, Alfred: Handbuch... Nacht 1 . [2.]. Alle seit Dezbr. 1884 his März 1886 erschienenen Postmarken etc. enthaltend, bearb. von W. Herrmann. Ebda. 1886. S. 597-701 $+183-193$ m. Abb. $8^{\circ}$.

- 6. Aufl. Neubearb.von Richard Senf, Leipzig. Ebda. $1888[-1890$.] 509 S. 8".

[Ureprünglich in 6 einzelnen Teileu veröffentlicht.]

- Nachtrag zu Bd. I von Dr. M.'s Handbuch für Postmarken-Sammler. 6. Aufl. Neubeart. von Richard Senf, Leipzig. Ebda. 1890. 55 S. $8^{\circ}$.

[Eineeitig bedruokt.]

- Handbuch für Postwertzeichen-Sammler. 7. Aufl. Bd. 1: Ill. Katalog aller bis zur neuesten Zeit erschienenen Postkarten [= Druckfehler, muB "Postmarken" heissen], Briefumschläge u. Streifbänder, [Sondertitel:] Handbuch sämtl. Postmarken, Briefumschläge u. Streifbänder. Bearb. von Richard Senf. Mit vielen erläuternden Anm. u, zahlr. Abb. Die beigesetztenVerkau fspreise sind die der Briefmarkenhandlung Gebrüder Senf in Leipzig. Leipzig: Gebr. Senf (1891.) XVI, 632 S. $8^{\circ}$.

- - Nachtrag. Alle bis Anfang 1896 hrsg. Postmarken, Briefumschläge u. Streifbänder enthaltend. Ebda. 1896.154 S. $8^{\circ}$.

- - Bd. 2. Illustrierter liatalog aller bis zur neuesten Zeit erschienenen Postkarten, Kartenbriefe, Postanweisungen und PaketBegleitadressen. [Sondertitel:] Handbuch sämtlicher Postkarten, Kartenbriefe, Postanweisungen und Paket-Begleitadressen. Bearbeitet von Theodor Haas. Mit vielen crlïuternden Anmerkungen usw. Leipzig: Gebr. Senf (1891). V1, 330 S. $8^{\circ}$

- Katalog über alle seit 1818 bis April 1874 ausgegebenen Briefmarken, Briefcouverts, Postkarten, Streifbänder und Lokalmarken aller Länder. Mlit beigedruckten Verkaufspreisen. Leipzig: H. Werninck \& Co. 1874. 74 S. u. 4 Tafeln m. Abb. $4^{0}$.

[Die 2. u. ff. Aufl eiehe u. d. T.: Moschkau, A1fred: Handbuch für Poetmarkensammler".]

- Katalog der vom Wiener Philatelisten-Club veranstalteten ersten öffentlichen Ausstellung der Postwerthzeichen aller Länder in Wien vom 13. biв 20. Novbr. 1881. Zsgest. von Dr. A. M., Secretär des Ausstellungs-Comité. Wien: Siegmund Friedl 1881. 8 S. $8^{\circ}$.

- Z Zeite Aufl. Ebda. 1881. 8 S. $8^{\circ}$.

- Deutscher Philatelisten-Kalender für 1877. Leipzig: Louis Senf $1877.52 \mathrm{~S} .8^{\circ} .\left(64^{\circ}\right)$

- Dr. von Stephan in Oybin (Juli 1892). Fin Gedenkblatt. Oybin: [Selbstverl.] 1892. $8 \mathrm{~S}$. $8^{0}\left(16^{\circ}\right)$.

[Nur in 25 numerierten Exemplaren verauegabt.]

- Photographisches Souvenir für Deutschlands Philatelisten. $6 \mathrm{Bl}$. in Visitformat. Nossen: Moschkau \& König, photogr.-art. Institut 1875.

B1. 1. Erinnerungen an 1870-71.

2. Die Essais Norddeutschlands.

"3. Correspondenzkarten-Tableau.

4. Postmarken-Tableau.
B1. 5. Couvert-Tableau

6. Philatelistische Literatur u. deren Förderer.

[Blatt 6 auch in Kabinett-Format erschienen.]

Mosehkau, Alfred: Die Wasserzeichen auf den seit 1818 bis dato emittirten Briefmarken $\mathrm{u}$. Couverts nebst Abriss einer Geschichte der Briefmarken u. des Briefmarkensammelwesens (.Philatelie": Mit 70 in den Text gedr. Holzschnitten. Dresden: C. E. Dietze 1871. VIII, 43 S. $8^{\circ}$.

(Philatelistiache Bibliothek Bd. 1.)

- Neue Aufl. Ebda. 1872. VIII, 49 S. m. $84 \mathrm{Abb}$. u. 1 Portr. Rowland Hills. $8^{\circ}$.

- Dritte gänzl. umgearb. Aufl. u. d. T.: Geschichte der Briefmarken n. der Philatelie (Briefmarkenkunde). Nebst einer Beschreibung aller bekannten Wasserzeichen auf Briefmarken, Couverts, etc. Leipzig: Louis Senf 1878. 88 S. u. 12 Taf. $8^{0}$.

(Philatelistische Bibliothek Bd, 1)

Vierte Aufl.u.d. T.: Die Wasserzeichen auf Briefmarken, Couverts, Postkarten etc. Nebst einer Geschichte der Briefmarken $\mathrm{n}$. des Briefmarkensammelwesens (Philatelie). F.bda. 1880. 98 S. u. 12 Taf. $8^{\circ}$.

(Philatelietieche Bibliothek Bd. 1.)

- Die Weihe der "Moschkau-Denktafel " im Hausgrunde am Oybin. Am 31. Mai 1882. [?] $1882.4 \mathrm{~S}, 8^{\circ}$.

- s. a. Antoniades, J. T.; Hruby, Robert; Oppenfeld, E. von; Raditsch \& Co., G.

Mossaner, Johann: Mitteilungen über unbekannt gebliebene frauzösische Postwertzeichen aus den Jahren 1850-1854. Gratisausgabe. StraBburg: [Selbstverl.] 1896. 12+2 S. $8^{\circ}$.

Motz, Ilermann: Missstände in der Philatelie. Vortrag. s. Deutscher Philatelistentag; in: Stenograpb. Bericht ü. d. Verbandlungen des Vll. D. Pl. zu Mannheim 1895.

Miilhausen i. Elsaß. (Internationale Postwertzeichen-Ausstellung.) Katalog zur I. Internationalen Postwertzeichen-Ausstellung Mülhausen i. Els. vom 12. bis 16. April 1903, abgebalten durch die BriefmarkensammlerVereine "Oberelsässischer Philatelistenclub" u. "Philatelisten - Verein Mülhausen*. Im Neuen Museum. Mülhausen i. Els.: [Ausstellungsleitung] 1903. $32 \mathrm{~S} .8^{\circ}$.

Miiller, A.: Zusammenstellung der wichtigsten Feldpost-Vorschriften für die Sendungen nach dem Feldheer $\mathrm{u}$. an deutsche Kriegsgefangene im Ausland. Weinheim: F. Ackermann 1914. $16 \mathrm{~S} .8^{\circ}$.

Miiller, Hans s. Die schwarze Liste des Vertraul. Korrespondenz Blattes 1891-1899.

[Bearheiter derselben.]

Miiller, H[aus] von: Zur Lebensgeschichte des Generalpostdirektors Schmückert, geb. 12. Novbr. 1790; gest. 3. Febr. 1862. Berlin: E. S. Mittler 1904.126 S. m. 3 Lichtdr. $8^{\circ}$.

Mïnchen (Bayerischer Briefmarken-SammlerVerein) s. Bayern. Neudruck. 
Mïnchen. (Bayerischer Philatelisten-Verein). Entwurf zur Finrichtung einer neuen Kaufvereinigung. München: Selbstverla 1884. 4 S. $4^{\circ}$.

[Autogranhiert.]

- Haas, Theodor: Katalog der vom B. Ph.-V. M. in der ersten October-Hälfte 1884 veranstalteten Postwerthzejchen-Ausstellung. München 1884.

- - - Vortrag über das Aufdrucksammeln. München 1885.

- Katalog zu der vom Bayerischen Philatelisten-Verein München veranstalteten 11 . lnternationalen Postwerthzeichen - Ausstellung, vom 29. Septbr. bis 8. Oktbr. 1889. München; [Ausstellungsleitung] 1839. 35 S. $8^{\circ}$. - Klenze, Heinrich von: Referat hetr. Agitation gegen das Aufdruckunwesen. München 1885.

- _ - Referat betr. Reform der Vordruckalben. Nünchen 1885.

- (Senf, Gebrüder:) Erinnerung an die 11. Internationale Postwertzeichen-Ausstellung des Bayerischen Philatelisten-Vereins München 1889. Leipzig [1889].

- - s. a. "Vertrauliche Mitteilungen" und ,Vereinsmitteilungen des Bayerischen Philatelisten-Vereins M̂̉nchen" Abteilung "Zeitschriften *.

Munderking s. Postgeschichte.

Mnnicipal-Stempelmarken Italiens s. Doczkalick, E.

Minster (Fürstbistum). ('ieschichte des Postwesens. s. Rensing, Franz Josef.

Niinzen-, Siegel-u. Briefmarken-Sammler, Der. 8. Ortleb.

Museu m, Literarisches. s. Literarisches Museum.

Nabha. Heitmann, Ernst: Nabha; im , GroBen Handbuch der Philatelie" Bd. 3. S. 1-13.

Nachseblagebuch, Philatelistiscbes. s. IVenzel, Ernst.

Nachweis philatelistischer Aufsätze in don vorhandenen Zeitschriften; in: Jahresbericht des Vereins für Briefmarkenkunde Kiol 1898/99. S. 37-60. Zsgest. vom Vereins-Vorstande. Kiel 1900. Druck von Schmidt \& Klaunig. $8^{\circ}$.

Nandgaon. Heitmann, Ernst: Nandgáum; im "GroBen Handbuch der Philatelie Bd. 3. S. $15-17$.

Natal. Tamsen, Emil und Frhr. von Scbell: Natal; im "Großen Handbuch der Philatelie" Bd. 3. S. $19-45$.

Nawanagar. Kalckhoff, Franz: Nawanagar; im ,Großen Handbuch der Philatelie" Bd. 3. S. $47-52$.

Nawiasky, Hans: Deutsches u. österreichis ches Postrecht. Der Sachverkehr. Erster Teil: Die allgemeine Rechtsstellung der Post. Wien 1909. 282 S. $8^{\circ}$.

[Ein vorzügl.Buch, auch dieBriefmarken behandelnd.]

Neapel. Diena, Emilio: Neapel; im ,Großen Handbuch der Philatelie" Bd. 3. S. 53-58.
Neapel. Neapel 1860 mit savoyischem Kreuz (Eälschung); in: Moschlzau, A., Zur Geschichte der Philatelie.

Segri-Sembilan. Heitmann, Ernst: NegriSembilan; im , GroBen IIandbuch der Philatelie- Bd. 3. S. 59 u. 60 .

Neilharl, Otto (x.: Uber den Wert des Sammelns von Marken auf Brief. Vortrag. s. Deutscher Philatelistentag; in: Stenograph. Bericht über den 5. D. Ph. in Berlin 1893.

Vepal. Heitmann, Ernst u. Franz Kalckhoff: Nepal; im "Großen IIandbuch der Philatelie* Bd. 3. S, $63-65$.

Neu-Braunschweig. King, Donald, A.: NeuBrannschweig; im ,Großen Handbuch der Pluilatelie $^{6}$ Bd. 3. S. $67-71$.

NeurCaledonien. Flandrin, Victor: Neu-Kialedonien; im "Großen Handhuch der Philatelie Bd. 3. S. $111-120$.

Neudrucke. Brummer, Leon: Über die bayerischen Neudrucke. Vortrag. s. Deutscher Philatelisteutag; in: Stenograph. Bericht ii. d. Verhandlgn. des 6. D. Ph. in Kiel 1894. Haas, Theodor: Über die angeblichen hayrischen Neudrucke. Vortrag. s. Deutscher Philatelistentag; in: Stenograph. Bericht über den 5. D. Ph. in Berlin 1893.

- Kalckhoff, Franz: Illustr. Verzeichniss aller bekannten Neudrucke staatlicher Postwerth. zeichen. Leipzig 1892.

- Ohrt, P[aul]: Handbuch aller bekannten Neudrucke staatlicher Postfreimarken und Ganzsachen. Leipzig 1806.

- - Zweck und Ausfühbbarkeit der Abstem. pelung von Neudrucken u. Fälschungen; in : Krötzsch, Hugo: Permanentes Handbuch der Postfreimarkenkunde. Vierteljabrs-Nachträge Jg. 1, Nr. 3. Johannis 1894. S. 41-55. $8^{\circ}$.

- Sellschopp, W.: Postwertzeichen, Neudruck und Sonderausgabe. Vortrag. Hamburg 1909.

- 8. a. Fälschungen u. Neudrucke.

Nendruekfrage. Dister, Georg: Zur Neudruckfrage. Essea 1909.

- Krötzsch, Hugo: Bericht über die Neudruckfrage. Vortrag, geh. auf dem XXl. Deutschen Philatelisten-Tag zu Karlsbad 1909.

Nene Berliner 0muibus- u. Packetfaht. Aetien-Ciesellschaft. 8. Tarif und Beförderungs Bestimmungen.

Neue Europa-Preisliste. s. Ruhen, Edvard Ml: Briefmarkenpreisliste $\mathrm{Nr} .15$.

Neue Republik. Tamsen, Emil u. Frhr. von Schell: Neue Republik; im ,GroBen Handbuch der Philatelie" Bd. 3. "S. $73-88$.

Yeu-Fundland. King, Donald, A.: Neu-Fundland; im "GroBen Handbuch der Philatelie* Bd. 3. S. $89-110$.

Neuheitenbesorgung. Borek, Richard: Neuheiten-Preisliste. s. Abt. Zeitschriften.

Pallausch, Z. J.: Zur Reform der Neubeitenbesorgung. Vortrag, gehalten auf d. XIV. Deutschen Philatelistentag in Wien 1902. 
Neulinger, Eduard ron: Permanenter Briefmarken-Katalog. Österreich-Ungarn. Mähr.Ostrau [K. C. Sauer 1889]. $8^{\circ}$.

- Schweiz. Ebda. 1890. $8^{0}$.

- Special-Katalog sämmtlicher Brief- und Zeitungs-Marken von Österreich - Ungarn. a. Oesterreich. b. Ungarn. c. Lombardei u. Venetien. d. Bosnien u. Herzegowina. Zsgest. von E. v. N. Wien: Sigmund Friedl 1858. $32 \mathrm{~S} 8^{\circ}$.

Neumann, Ileinricl L., K. u. k. Hofkunsthändler, Wien. (Versteigerung seiner Europa-Sammlung.) s. Auktions-Kataloge, K. k. Versteigerungsamt Dorotheum, Wien. 261. Kunstauktion.

Yeu-Sehottland. King, Donald, A.: Neu-Schottland; im "Großen Handbuch der Philatelie" Bd. 3. S. $123-130$.

Neuseeland. Sachs, Franz: Die Dienstganzsachen Neuseelands. Berlin 1913.

Niederlande. Kohl, Paul: Illustr. Katalog der Freimarken von Europa. Abt. 2. "Niederlande ${ }^{4}$. Chemnitz 1894/95.

- Moens, J. B.: Niederiändische Essais. Berlin 1898.

- Sauer, K. Cl.: Permanenter BriefmarkenKatalog. "Niederlande u. seine Colonien." Mährisch-Ostrau [1889].

Niggl, Arthur: Das Postrecht. Die wichtigsten Vorschriften des inländ. u. des internationalen Postrechts mit Erläuterungen. Stuttgart: W. Kohlhammer 1913. V11, 267 u. 7 S. $8^{\circ}$.

- - (Neue Aufl.) Die Vorschriften des inländischen u. internationalen Postrechts (mit Einschluss des Postscheckrechts). Erläutert. Ebda. 19I4. VII, 306 S. $8^{\circ}$.

No. 1. I'hilatelisteu-Club st. Gallen. s. Pfenninger, Otto: Handbuch der Schweizer Postwerth-Zeichen. Lausanne 1890.

Nordborneo. Lindenberg, Carl: Britisch-NordBorneo; in "Großen Handbuch der Philatelie Bd. 1. S. 252-256. Leipzig 1888.

Norddentscher Postbezirk. Die Essais des norddeutschen Bundes. Photographisches Tableau mit 32 Photographien. Berlin: Selbstverl. des Generalpostamts 1867.

- Gobbert, Fr.: Verzeichnis sämmtlicher amtlich ausgegebenen Briefmarken der staatı u des deutschen Bundes bis zur Errichtung des deutschen Reiches. Breslau 1891.

- Kalckhofi, Franz: Die Erfindung der Postkarte u. die Korrespondenzkarten der Norddeutschen Bundespost. Leipzig 1911.

- König, Emil: Deutschlands Feldpost. Gera 1871.

- Krötzsch, Hugo: Norddeutscher Postbezirk 〈nebst Feldpost 1870/71). Leipzig 1895.

(Krötzecb, Handb. Bd. 11.)

- Lindentserg, Carl: Die Briefumschläge des Norddeutschen Postbezirks. Berlin 1893.
Norddeutseher Postbezirk. Moschkau, A.: Photographisches Souvenir für Deutschlands Philatelisten. Bl. 2. Die Essais Norddeutschlands. Nossen 1875.

- Postrerwaltung. Verordnungen und Amtliche Nachrichten für H'lsass-Lothringen aus der Zeit vom Beginn der deutschen Occupation bis Ende Mlärz 1872. Hrsg. vom Oberpräsidial-Büreau. Strassburg: Karl J. Trübner 1872. I, 592 S. $8^{\circ}$.

Tordisches Briefmarken-Depot. Preisliste Nr. 1. Copenhagen: [Selbstverl.] Juni 1875. 2 S. $4^{0}$.

\section{- - Nr. 2. Ebda. August 1875. 4 S. $8^{\circ}$.}

Normal-Katalog, Neuer, für Briefmarken. (N. N. K.) 1914. Red.: Paul Kohl. BerlinSchöneberg: Neuer Normal-Katalog (1913). $\mathrm{X} \backslash \mathrm{X} 1,1119 \mathrm{~S}$. Leinw. geb. $8^{0}$.

[Anfang e. n. d. T. "Briefmarken-Normal-Katalog, Illustrierter".]

- (N. N. K.) 1915. Red.: Paul Kohl. Fbda. 1915. XXXIl, 1224 S. Leinw. geb. $8^{\circ}$.

- - (Nachtrag 1.) Kriegsmarken von Deutsch= land, Österreich-Ungarn und den neutralen Staaten. Ebda. 1915. 4 S. $8^{0}$.

- - (Nachtrag 2.) Kriegsmarken von Deutschland, Österreich-Ungarn und den weutralen Staaten, sowie rerschiedene durch den lírieg hervorgerufene Marken. Ebda. 1915. 8 S. $8^{0}$. [Forteetz, erscheint nicht mehr.]

Norwegen. Andresen, Ferd. jr.: Engros. En detail. Preisliste über norwegische Briefmarken. Christiania 1896.

Preisliste über Skandinavische Briefmarken. Christiania 1897.

- Kohl, Paul: Illustr. Katalog der Freimarken von Europa. Abt. 4. "Norwegen". Chemnitz $1894 / 95$.

Rommel, Otto: Die Privat-, Eisenbahn. u. Dampfschiffsmarken von Skandinavien und Finnland. Gössnitz 1909.

- Ruben, M. E.: Vollständige Preisliste von ... Norwegen ... Kopenhagen 1889/90.

Nïrnberg. (Bayerische Landes-Industrie-Gewerbe- und Kunst-Ausstellung in Nürnberg.) Briefmarken. Separat-Abdruck aus den Nummern 423 u. 425 des "Korrespondenten von und für Deutschland", Nürnberg. [1888.] 16 S. $8^{\circ}$.

Niissle, Dr.: Alfred Moschkau, Biographie; in: Moschkau, A.: Handbuch für PostmarkenSammler. 2. Aufl. Leipzig 1876.

Oberelsässiseher Philatelistenelub. s. Mül. hausen i./FisaB (Internationale Postwertzeichen Ausstellung).

(limann, Fritz: Die Anfänge des Postwesens u. die 'Taxis. Leipzig: Duncker \& Humblot 1909. XI, 342 S. m. 1 Stammtaf. u. 1 Karte. $4^{0}\left(\right.$ gr. $\left.8^{\circ}\right)$.

0elımigke \& Riemsehneider: Briefmarkenbilderbogen. 120 bunte Abbildungen. NeuRuppin 0. J. (um 1872).

[Nr 5146 der Neu-Ruppiner Bilderbogen; ee erecbienen auber dienem noch melrere Bogen.] 
Ohrt, l'aul: Fintutafelu. Fiir I'ostwertzeichen Sammler. Hrsg. v. P. U. .. | Hraunschweig. (Germania-King", Verband Dentscher l'ostwertzeichensammler - Vereine.) GermaniaHandlücher $\mathrm{Bd}$. 1.] Düsseldorf 1906 . IV 10 S. u. 33 Taf. i. Nappe. $4^{\circ}$.

- Philatelistisches Feigenblatt I: Zur Vertretung von Sammlerinteressen. Filzpariser oder Bismarck'sche Kürassierstiefel? Philatelistisch-satirische Monographie usw. Dïsseldorf: Selbstverl. d. Verf. 1908. 8S. $8^{\circ}$.

- Philatelistisches Feigenblatt II: [Ist nicht ersehienen.]

- Philatelistisches Feigenblatt 11I: Kosackenfrage und Russenplage. Werden die Marken der deutschen Schutzgebiete philatelistische Schmutzgebiete? Diisseldorf: Selbstverl. d. Verf. 8 S. $8^{\circ}$.

[Ist vicht zur Verbendung gelangt; 6. a. Kuback, Philipy: Philatelist. Feigenblait III.]

- Handbuch aller bekannten Neudrucke staatlicher Postfreimarken, Ganzsachen u. Essays, nebst Angabe ihrer Kennzeichen. Unter Mitwirkung hervorragender Sammler u. Händler zsgest. von P. O. [Braunschweig. ( GermaniaRing ${ }^{*}$, Verband Deutscher Postwertzeichensammler - Vereine.) Germania-Handbücher Bd. 2.] In Lfgn. $8^{\circ}$.

T. 1. Afghanistan-Hamburg. Düsseldorf 1907. S. $1-125$.

2. Hannover-Marshallinseln. Düsseldorf 1912. S. $126-261$.

3. [Noch unvollendet.] S. $261-$

[Das pleiche Werk erscheint außerdem noch seit 1906 in zwanglosen Lieferungen ala Beilage zum n1lluetr. Briefmarken-Journal ${ }^{\star}$. Das Titelblatt trigt hierzu den Aufdruck ${ }_{n}$ Germanja-Handbuch ${ }^{*}$. Leipzig: Gebr. Senf iu Komm, 1906. Desgl, noch unvollendet.]

- Moldau 27 parale. (Beilage zu ,GermaniaBerichte 2].9. 1900.) [Charlottenburg: Selbstverl. 1900.] 4 S. u. 1 Kunstdr.-Taf. $4^{0}$.

- Notizen über Hamburger Postfreimarken. Separatdruck aus den Germania-Berichten. [Charlottenburg: Selbstrerl. 1900.] 4 S. $8^{\circ}$.

Oldenburg. (Postmarken.) Leipzig: Ḱrötasch 1894. 120 S. u. 18 Taf. $8^{\circ}$.

(Krötzech: Handb. Bd. 12.)

Photographie und Projektion für philatelistische Zwecke. Vortrag. 5. Deutscher Philatelistentag; in: Stenograph. Bericht ü. den XV. Deutschen Philatelisten-Tag ... in Pforzheim 1903.

- Die Poststempel von Oldenburg.

[Erscheint seit 1913 in bogetweieen Lfgu. zu je 16 Seiten $8^{\circ}$ fortlaufend al Beilage zuden Mitheilungen der Firma Paul Kohl, G. m, b. Il. Chemujtz u. isl noch unvollendet.]

- Preussen. (Postmarken.) Leipzig: Krötzsch 1896. 23 \& S. v. 14 Taf. $8^{\circ}$.

(Ǩ̈rützeh: Handb. Bd. 13.)

- Die hamburgischen Steindruck-Marken (I'/ u. $21 / 2$ Schilling) der ungezähnten Ausgabe 1864. (Sonderdr. aus der Festschrift z. Feier des zehnjährigen Bestehens des Berliner Philatelisten-Klub.) Berlin: Selbstverlag des B. Ph.-Kl. 1898. 18 S. u. I Taf. m. Abb. $4^{\circ}$. - s. a. Berlin (Berliner Philatelisten-Kilub). Festschrift zur Feier des zehnjährigen Bestehens des B. Ph. Kl.
Ofitenburg. Lindenberg, Curl: Dio Briefunschläge von Oldenburg. Berlin 1893.

Ohrt, Paul: Oldenlurg (l’ostmarken.) leeipzig $\mathbf{1 8 9 4 .}$

- Die Poststempel von Oldenburg. Cliem nitz 1913

(Jpitz, C. d Paul Lederer: Lücke's Länderund Weltverkehrskarte für PostwortzeichenSammler mit Darstellung der Lage aller Mlarken ausgebenden Länder nebst Beriicksichtigung des gesamten Weltverkehrs. Bearb. von C. O. u. Paul Lederer. Leipzig: C. F. Lücke [1904]. gr. $2^{0}$ (Oblong.).

Oppenfeld, E. won: Manco-Jiste nach der fünften Auflage von Dr. Moschliau's Handbuch für Postmarken-Sammler. (Leipzig 1883.) Bearb. von F. v. O. Berlin 1883. IV, $153+3$ S. $8^{\circ}$.

Orient. Irataloge und Preisverzeichnisse über Orientalische ${ }^{4}$ Postwertzeichen. S. Antoniades, J. T.; Hruby, R.; Marcus, M. H.; Melkenstein \& Photiadès; Photiadès, C. D.; Tavoukdji.

Ortleb: Der Münzen-, Siegel- und Briefmarken-Sammler. Berlin: S. Mode's Verlag 1885. $46 \mathrm{~S} .8^{\circ}$.

isterreichischer Philatelisten-Klub, .Yindobona*. s. Wien: Osterr. Philatelisten.Klub "Vindobona".

Oisterreich-Ungarn. Bart], Johann: Handbuch für den ausübenden Postdienst iu Üsterreich. Auf Grund der bis Ende Dezlur. 1903 erschienenen Verordnungen und Erlässe bearb. u. hrsg. von Jos. Bart] u. Fr. Pelzl. 11. Aufl. Wien: Gerold \& Co. 1904. LXVI, 862 S. $8^{\circ}$

- Berger, H. [d. i. Arthur Wülbern]: Illustr. Preis-Katalog der 'Telegrafen- und Stempelmarken. Bd. 2. Österreich-Ungarn u. Balkanstaaten. Hamburg 1898.

- Derblich, Leo: Oesterreichisches Postrecht. Eine Sammlung der auf das Postwesen bezughabenden oesterreichischen Gesetze und Verordnungen, unter Anschluss von Entscheidungen des k. k. Verwaltungs-Gerichtshofes u. k. k. Obersten Gerichtshofes. Prag l, Postuasse 33: Finanz-Proc-Sekretär D. L. Derblich 1901. V11, I9I S. $8^{\circ}$.

- Eissen, Franz, \& Co.: Catalog sämmtlicher bis Anfang $\mathbf{1 8 7 9}$ erschienemen österreich. ungarischen Briefmarken, Couverts, Postkarten, Anweisungeu u. Nachnahmekarten, mit Preisangabe, Budapest [1879].

- (Friedl, Rudolf:) [Katalog der] X. Briefmarken-Auktion der Friedl'schen Spezialsammlung von Österreich-Ungarn, Lcmbardei, Lerante u. Bosnien. Auktion: 21. bis 25. Mai 1917. Schaustellung: 16.-.20. Mai 1917 in den Kunstauktionssälen des Dorotheums. Wien: K. k. Versteigerungsamt Dorotheum. 1917. 109 S. nebst 27 Illustr.Taf. auf Kunstdruckpapier. $4^{\circ}$.

- Friedl, Rudolf: Illustr. Spezialkatalog und Preisliste sämtlicher Post- u. Telegraphenwertzeichen v. Österreich-Ungarn. Wien 1897. 
Österreich - Ungarn... Friedl, Sigmund: \%or Geschichte der Osterreichischen Mereure 1850-56. Über die Österreichischen Mercure und die Prüfungs-Autoritäten derselben. Wien 1895.

- Hugelmann, Carl: Die Concurrenz der Wertzeichen in der Oesterreichischen Briefpost. Wien 1889.

- Joessel, Alfons: Die Postwertzeichen der Oesterreichisch-Ungarischen Monarchie. Leipzig 1898.

- Kohl, Paul: Illustr. Katalog der Freimarken von Furopa. Abt. 5. "Osterreich-Ungarn." Chemnitz 1894/95.

- König, Emil: Schwarze Kabinette. Mit Anlagen: Geschichte der 'I'hurn u. 'T'axis'schen Postanstalt und des österreichischen Postwesens. - liraunschweig 1875.

- Kropf, Hans: Die Abstempelungen der Marken von Österreich-Ungarn und Lombardei-Venetien ron 1850 bis 1858 . Prag 1899.

- Die Postwertzeichen der Österr.-ungar. Monarchie. Prag 1902.

-.. Die Postwertzeichen dos Kaisertumes Österreich $\mathbf{u}$. der österreichisch-ungarischen Monarchie. Prag 1903.

- Lengyel, S.: Die Wasserzeichen der ungarischen Postwerthzeichen. Leipzig 1890.

- Nawiasky, Hans: Doutsches u.österreichisches Postrecht. Der Sachverkehr. Wien 1909.

- Neulinger, Eduard von: Spezial-katalog von sämmtlichen Brief- u. Zeitungsmarken v. Österreich Ungarn (1850-1 888 ). Wien 1888.

- Prückler, J. C.: Katalog sämmtlicher bis Anfang 1886 erschienenen österreich-ungar. Postmarken etc. Budapest 1886.

- Radics, P. v.: Die k. k. Post in Krain und ibre geschichtliche Entwickelung. Laibach 1896.

- Woerz, Ritter Hans von: Über die Neudrucke der Marken von Österreich und der Lombardei. Vortrag. Wien 1911. (Hamburg 1912.)

- s. a. Graz (Verein der Briefmarkensammler). Festschrift des 50 jähr. Jubiläums der ersten Oesterreichischen Briefmarke.

ostfriesland. Postwesen. s. Esslinger, C.

Ostrumelien. Hraby, R.: Katalog aller türkischen, ostrumelischen und russischen levantepostmarken, Karten und Couverts mit Preisen. Constantinopel 1884-1891.

Ostwald, Wilhelm: Die Farbentafel mit 8 Zeichnungen u. 192 Farben. Leipzig: Verlag Unesma, G. m. b. H. 1917. 45 S.

Owiteh, Alexander: Welt-BriefmarkenhändlerAdressbuch. (त Universel-'T'imbre-Poste-Livre d'adresse. "Universal-Stamp dealer Adressbook" ...) Hamburg-Uhlenhorst: Selbstverl. des Verf. 1878. XIV, 94 S. $8^{\circ}$.

Pallausch, Z. J.: Zur Reform der Neubeitenbesorgung. 8. Deutscher Philatelistentag; in Stenograph. Bericht ü. d. XIV. D. Ph.... in Wien 1902 . lapiergeld, Amrikanisches postamtliches; in: Moscbliau, A, Kur Geschichte der L'hilatelie.

Papna. Kohl, Paul: Die lostwertzeichen von Papua. Chemnitz 1912.

Paris. (Internationale Postwertzeichen-Ausstellung 1900. Classe 111, Division l, Section B.) s. Lindlau, Joseph: Katalog zur Sammlung der Postwerthzeichen: "Altdeutsche Staaten". Heidelberg 1900.

l'auli, Emil: Entwfrtung falscher Marken. Erwiderungs-Rede geh. am 2. Septbr. 1906 auf dem XVIII. Deutschen Philatelistentag in Nürnberg. s. Deutscher Philatelistentag; in: Stenograph. Bericht ü. d. IVllI. Deutschen Philatelistentag... in Nürnberg 1906.

- Geschichte, Wesen u. Bedeutung der Deutschen thilatelistentage. In Erweiterung der in Hannover am 25. Deutschen Philatelistentage $\langle 18$. August 1913〉 gebaltenen Festrede dargestellt. Berin: Selbstverl.1913. 33 S. $8^{0}$.

-Die schwarzen Listen, Kataloge u. Bücher auf dem Gebiete der Philatelie im Lichte des Rechts. Vortrag geh.auf dem XV.Philatelistentage zu Pforzheim 1903. Greifswald: Hans Adler 1903. 27 S. $8^{\circ}$.

_ _ s. a. Deutscher Philatelistentag; in: Stenograph. Bericht über den XV. Deutschen Philatelistentag . . . in Pforzheim 1903.

- Über den strafrechtlichen Schutz der Postwertzeichen in Belgien gegen Fälschung $u$. Missbrauch im lichte der Philatelie. Sonderabdr. aus der Festschrift zum fünfundzwanzigjährigen Bestehen des Berliner PhilatelistenKlubs. Berlin: Selbstverl. 1913. 17 S. $8^{\circ}$.

- Über den strafrechtlichen Schutz der Postwertzeichen des Deutschen Reichspostgebietes gegen Fälschung und Missbrauch. Nebst einem Vorschlage im Interesse der Philatelie auf Abänderung der Paragraphen 284 v. 308 No. 3 des Vorentwurfs zu einem Deutschen Strafgesetzbuche unter vergleichender Be. rïcksichtigung der Vorentwïrfe zu den Strafgesetzlïchern für Österreich u. die Schweiz. Berlin: Selbstrerlag 1911. XVI, $101 \mathrm{~S}$.

- Zweck und Ziele sowie Entwicklungsgang der Wissenschaftlicben Gruppe. Vortrag. s. Deutscher Philatelistentag; in: Stenograph. Bericht des XXI. Deutschen Philatelisten'Tages... zu harlsbad 1909.

P'egan, Enrico: Adressbuch der hauptsächlichsten Briefmarken-Händler. Mit 100 Adressen. 1.-3. Aufl. Triest: Selbstverl. 1872. Je12S. $8^{\circ}$.

Peka-Album, Das. Ein Spezialalbum für Altdeutschland, Deutsches Reich mit Postämtern und Kolonien. Chemnitz: Paul KoblG.m.b.H. o. J. Prospekt $8 \mathrm{~S}$. auf Kunstdruckpapier m. Abb. $8^{\circ}$.

Penuy-I'orto-System. s. Finke, Georg: Geschichte des Yenny-Porto-Systems. Leipzig 1890. 
I'erlep, A.: Katalog der stempelmarken aller Staaten. Mit 45 Alub. u. beigesetzten billigsten Preisen, zu denen sowohl der Verleger dieses Kataloges dieselbeu besorgt, als auch durch jede andere Briefmarkenhandlung zu beziehen sind. Leipzig: Louis Senf 1880. 225 S. u. 3 Taf. $8^{\circ}$.

(Thilatelistische Bibliothek Bd, 6.)

l'ermanent-Katalog, Schwarzer. s. GermaniaRing.

1'ersieu. Schüller, Fr.: Die persische Post u. die Postrertzeichen von Persien u. Buchara. Wien 1893.

Peru. Dauth, H. J.: Catalogue de l'Union Postale Universelle. Abdruck postamtlicher Dokumente H. 2. Aufstellung sämtl. Postwertzeichen Perus.

- Gutmensch, J.: Peru [Katalog]. Fraukfurt a. Maiu [Brünu] 1887.

- Medio Ceso (Fälschung); in: Mosclikau, A., Zur Geschichte der Pbilatelie.

- Rommel, Otto: Studie äber die Postwerthzeichen von Peru. München 1890.

Petritz, Erust: Mauco-Liste zu Dr. Kloss's Karten-Catalog ... Dresden: Selbstverl. ¿. Hrsgs. II, 37 S. $8^{\circ}\left(24^{\circ}\right)$.

(s a. Kloss, Paul: Verzeichnis u. Feschrsibung sller Postkarten etc. "Zweite Ausg. ${ }^{\prime}$ )

Pfaff, Car]: Über Bergedorfer Briefmarken auf Grundlage amtlicher Bestimmungen. Hanuover: Selbstverl. [1891.] $10 \mathrm{~S} .8^{\circ}$.

Pfenninger, otto: Handbuch der Schweizer Postwerthzeichen mit Beigabe von postamt. lichen Erlassen. Hrsg, von No. 1. Philatelisten-Club St. Gallen unter dem Protektorate Schweiz. Philatel. Vereine zur Feier des 40. jährigen Jubiläums der ersten Schweizer Buudespost-Marken ... Lausanne: Hans Kirchhofer 1890. 135 S. m. Abb. $8^{\circ}$.

- Kantonale und schweizerische Postentwerthungsstempel aus den Jahren 1850-1854. Ebda. [1891.] 8 S. u. 5 Taf. m. 107 Abb. $8^{\circ}$.

Philatelia. Vereinsmitteilung. Sondershausen 1887. s. Laue, Robert.

Philatclie. Der Ballast in der Philatelie. Vortrag s. Glasewald, A. E.

- Einiges aus der Philatelie; in: Jeran, O.: Wegweiser f. Verkehrswertzeichen-Sammler (Philateliston-Fibel.)

lhilatelisten, Fürstliche; in: Moscbkau, A., Zur Geschichte der Philatelie.

l'hilatelisten-Adressbuch s. Haack, Hermann. (s. a. u. nAdressbìcher".)

philatelisten-Kalender s. Haack, Hermann.

- Deutscher, für das Jahr 1877. Leipzig: Louis Senf 1877. 50 S. $8^{\circ}$.

- Illustrierter, s. Dejhle, R.

Philatelisten-Klub St. Gallen s. u. No. 1.

Philatelistentage. Feltmann, H.: Anleitung zur AbhaItung von Bundes- und PhilatelistenTagen. Entworfen vou H. F. unter Mitwirkung der Herren Baumgarten, Dedecke und
Dr. Künkler. Gestiftet von Jos. Baumgarten IVien. Hannover 1914. Druck von Carl Eluers. 32 S. $8 \%$.

[Seiten 1-21 einseitig bedr.]

plibatelistentage. s. a. u. "Deutscher Plilatelistentag".

llillippinel, I. Fmission (Fälschung); in: Moschkau, A.: Zur Geschichte der Philatolie.

l'lotiades, C. 1). 1892-1893. Katalog über Orientalische Postwerthzeiclıen zsgest. nach Meyer's u. Moschkau's Handbucb für Postmarkensammler $\mathrm{u}$. mit billigsten Preisen versehen per Stück, Satz, 10, 100, 1000, 5000 u. 10000 Stück. Hrsg. von C. D. P. Consulat de Grèce. Constantiuople: Selbstverl. d. Verf. 1892. VI, $18 \mathrm{~S} .8^{\circ}$.

P'lotographie u. Projektion für philatelistisclıe Zweeke. Vortrag gehalt, auf dem XV. Deutschen Philatelisten-Tag ... in P'forzheim 1903. s. Ohrt, Paul.

Pilger, Robert: Helgoland und seine Post. (Aus "Deutsches Postarchiv".) Cuxharen 1875. $8 \mathrm{~S} .8^{\circ}$.

Pilzecker, 0tto: Preisbuch Nr. 1 der Briefmarkenbörse Pilzecker in Hannover. Fehlliste für Schwaneberger's, Schaubek's u. Zschiesches Sammelbuch. Hannover u. Leipzig: [Cruse 1889.] 140 S. $8^{\circ}$.

- Preisbuch Nr.2. Ebda. [1891.] 122 S. $8^{\circ}$.

Pirl, Paul: Ein Beitrag zur Abstempelungsfráge. Ein Rückblick, Umblick v. Ausblick. Charlottenburg: Selbstverl. [1911.] $11 \mathrm{~S} .8^{\circ}$. - Der 3 u. 20 Pf.-Wert von Helgoland im Original u. Neudruck. (Sonderdr. aus der Festschrift zur Feier des 25 jäbrigen Bestehens des Berliner Philatelisten-Klub.) Berlin: Selbstverl. des B. Ph.-Kl. 1913. 36 S. u. 6 Taf. m. Abb. $8^{0}$.

- s. a. Berlin (Berliner Philatelisten-Klub). Festschrift zur Feier des 25jährigen Bestehens.

- Leitsätze zur Bewertung der Abstempelungen. [Beilage zu den Germania-Berichten] Schutzblatt Nr. 2. Essen a. d. Rubr: Germania-Ring 1913. 4 S. m. Abb. $4^{0}$.

Pleimes, Alphonse: Engros-Preiskurant von allen Marken von Sudan u. Aegypten. Birket-El-Sab (Aegypten): Selbstverl. 1911. $8^{\circ}$.

Polen. Kohl, Paul: Illustr. Katalog der Freimarken von Europa. Abt. 4 "Polen". Chemnitz $1894 / 95$.

- Krause, Richard: Die Philatelie in Polen. in: Philatelia. Sondershausen 1887.

Popularitït des Briefmarkensammelns im Pubtikum zu fördern. s. Scheibe, Max.

Portugal. Berger, Ludwig: Portugal. Studie über die Ausgaben 1853-1876. BerTin 1898.

- Genth, F.: Abstempelung u. Entwertung auf Marken Portugals u. seiner Kolonien. Chemnitz 1911.

- Gutmensch, J.: Permanenter BriefmarkenKatalog. Portugal u. seine Colonien. Mährisch-Ostrau [1888]. 
Portngal. Kohl, Paul: Illustr. Katalog der Freimarken von Europa. Abt. 9 "Portugal". Chemnitz 1894/95.

- Nangold, Otto: Catalogue de l'Uuion Postale Universelle. Abdruck von den Generalpostämtern überlassener Schriftstücke. Hrsg. v. I1. .T. Dauth. Amtl. Liste: Portugal u. Colonien. Frankfurt a/N1. [1891].

- Sauer, K. Cl.: Permanenter Katalog. Portugal u. Colonien". Mährisch Ostrau [1887].

Post. Die Post im Auslande s. Siebelist, O.

- Die Post u. Telegrapbie in Hamburg. Denkschrift zur kinweihung des neuen ReichsPost- u. T'elegraphen-Gebäudes am Stephansplatz. Hamburg: J. Ronge 1887. VI, 55 S. u. 1 Taf. $8^{\circ}$.

- Die Post u. Telegraphie auf Helgoland Heligoland] 1895. II, ]8 S. $8^{\circ}\left(16^{\circ}\right)$.

[Umschlagt.: "Das neue Posthaus auf Helgoland". Eröffnung im September 1895.]

- Die Post der Urzeit s. Jacobsen, Nils: Die Zeitbücher der Weltpost. Leipzig 1892.

- Die Post auf dem Weltmeer s. Klaus, O.

Postalieus, Jocosus: Die Post sonst und jetzt. Historiscbe-panegy rische Ver'se. Insterburg: B. E. König 1881. 44 S. $8^{\circ}\left(16^{\circ}\right)$.

Postiimter, Fliegende. (Aus dem Buche von der Weltpost.) in: Isakovics, A. v.: Intern. Tausch-, Adress- u. Hülfsbuch f. d. Tauschverkehr. T. 2.

postluch für Berlin und Umgegend. Hrsg. von der Kaiserl. Oberpostdirektion in Berlin. Berlin. Gedr, in der Reichsdruckerei. $8^{\circ}$.

[Erschien von 1874-1879 n. d. T.: Postbuch fir das correspoudirende Publikum in Berlin, ab 1879 bis 1913 auch u. d. T.: Postbuch z.11m Gebrauch für das Publikum iu Berlin (u. Uragegond). Alunliche Postbücher sind für Baden, Breslau, Cöln, Dresden, Düsseldorf, Hamburg und wabrscheinlich noch für andere Verkehrsmittelpunkte orschienen].

Postlmeh für München. Hrsg. rom Verkehr'samt der K. b. Posten u. Telegraphen in München. Stand vom 1. 5. 1914. Nünchen: H. Lukaschik 1914. I1, V, 176 S. $8^{\circ}$.

Postburean. Französisches Postbureau zur Zeit Ludwig XV. in: Isakovics, A. v.: Internationales Tausch-, Adress- u. Hülfsbuch f. d. Tauschverkehr. T. 2.

Post-Formularbncli, Illustrirtes. Entbält die im Verkehr mit der Post. vorkommenden Formulare u. deren Ausfüllung, sowie die Art der hauptsächlichen Aufschriften bei den Versendungsgegenständen. (Gratis-Beil. zu "Henze's Illustr. Anzeiger.") NeustadtLeipzig: Commissionsrath Henze 1882. $16 \mathrm{~S}$. m. tarb. Abb. $4^{\circ}$.

P'ostgebühren, Die neuen. [Plakat.] (1 Bl. auf Pappe) $21,5 \times 19 \mathrm{~cm}$. Leipzig: Meister \& Schirmer [1916].

- - gültig vom I. VIII. 1916 ab. [Plakat.] (1 Bl. auf Pappe). Leipzig: P. M. Blüher [1916.] $8^{\circ}$.

- 4 Bl. in Leporelloform. $9 \times 6 \mathrm{~cm}$. [0. 0 . u. n. J.] (Wiesbaden: H. Giess 1916.) l'ost- u. Telegrammgebiiluen, Die neuen, am 1. VIII. 1916 in Kraft tretend. (1 Bl. auf Karton.) 19,5 $\times 23,5 \mathrm{~cm}$. Dresden: Buchdr. von M. E. Fiseher [1916].

Postgebiihren-0rduung, Die neve, für das Gebiet der Reichspost, Bayern u. Württem. berg, Generalgouveruement Warschau u. im Ftappengebiet des Oberhefehlshabers Ost sowie Osterreich-Ungarn, Luxemburg u. den wichtigsten Auslandsverkehr. Nach amtl. Augaben. Gültig vom 1. VIll. 1916 an. Reutlingen: R. Bardtenschlager [1916]. 6 S. in Leporelloform. $8^{\circ}$.

Postgebiihrentabelle. s. Weber, A.

Postgeselichte, 'Hoclinteressante Munderkinger, aus den Jahren 1870, 1872, 1873, 1894-1896. Stuttgart: Strecker \& Moser 1896. 74 S. $8^{0}$.

Postmann, T.: Feldpostdienstordnung u. Ausführungsbestimmungen, sowie die Feldpostrorschriften für den Posthetriebsdienst in gedrängter Darstellung, nebst Anh.: Repetitorium in Frage $u$. Antwort. Frankfurt a./M.: Hermann Ehrentraut 1910. 59 S. $8^{\circ}$.

I’ostmarken-Tal)leau. s. Moschkau, A.: Photographisches Souvenir für Deutschlands Philatelisten. Bl, 4. Postmarken-Tableau. Nossen 1875.

Postreform. Speiser, W.: Von der Feldpost zur Postreform. Leipzig 1915.

l'oststammbuch. Eine Sammlung vou Liedern u. Gedichten, Aufsätzen u. Schilderungen, gewidmet den Angehörigen u. Freunden der Post. 3. verm. u. m. Abb. versehene Ausgabe. Berlin: Kgl. Geh. Oher-Hofbuchdr. (R. v. Decker) 1877. XIX, 248 S. $8^{0}$.

loststrassen. Karte der deutschen Poststrassen in Frankreich 1870/71. s. Krötzsch, Hugo.

I'ost-T'arif, Neuer, ab 1. August 1916 mit besonderer Berücksichtigung des württ. Postverkehrs. Stuttgart: Paul Mähler [1916]. $4 \mathrm{~S} .8^{\circ}$.

Postwertzeichen - Ausstellungen. s. Berlin; Bern; Leipzig (Intern. Ausstellung für Buchgewerbe u. Graphik 1914); München; Paris; iVien.

l'ostwertzeichen-Kataloge (allgemein.Inhalts). Baumbach \& Co., Bausebke, Gustav; Brand, H.; Briefmarhen-Nolmal-Katalog; Dannenfelser, W. F.; Friedl, Sigmund; Gast, Richard; Georg, Wilhelm; Glasewald, A. E.; Grossmann, E. W.; Hayn, Ernst; Heitmann, Ernst (Handhuch); Hirt, A.; Joseph, Dr.; IKloss, Paul; Kohl, Paul; Kosack, Philipp; Kümmel, Julius; Künast, Walther; Larisch, Anselm; Lauber, August; Lietzow, Paul (Handbuch); Lindenberg, Carl; Literarisches Museum; Lublin, G.; Maier, N. Kurt; Mann, Christian; Marken-u. Gauzsachenhaus G. m. b. H.; Meyer, Ferd.; Michel, Hugo; Moschkau, Alfred; NormaIKatalog, Neuer; Priebatsch, Leopold; Prietsch, F. B.; Ruben, Edvard N.; Dartori, Georg; 
Sauer, Karl, Cl.; Schaubek, Gustav; Schilling, C. E.; Schmidt, S.; Schroeder, Oswald; Schubert, G. W.; Schultze, Joh. O.; Seelig, G.; Senf, Gebrüder; Senf, Louis; Siekmann \& Braunschweig; Thiele \& Co.; Tramburg's lirhen; Ulex, Oskar; Vedel, M.; Werninck, H. \& Co.; Willadt, Carl; Willadt, Carl, \& Co; Wuttig, G.: Zschiesche, Alwin; Zschiesche, Reinherz; Zschiesche \& Köder.

Postwertzeichen-Kataloges. a. Berlin (Reichspostmuseum).

- s. a. Sachs, Franz: Ein Vergleich der Preise der massgebenden Kataloge. Berlin 1913.

Postwesen. Aschenborn, M.: Das Gesetz über das Postwesen des Deutschen Reichs rom 28. Oktoher 1871 und die Vorschriften der Reichsverfassung üher das Post- und 'T'elegraphenwesen. Art. 48-52. Erlïutert von M. A. Berlin: Julius Springer. 1908. V111, 429 S. $4^{0}\left(\mathrm{gr}^{\circ} 8^{\circ}\right)$.

- Becker, Herm. Joseph: Das Postwesen im Saargebiet. Sacrbrücken 1915.

- Dambach, Otto: Das Gesetz ïber das Postwesen des Deutschen Reichs vom 28. Oktober 1871. 6. vєrm. u. veränderte Aufl. hrsg. von Dr. Ernst von Grimm. Berlin: R. Schoetz 1901. XXIV, 364 S. $4^{0}$ (gr. $8^{\circ}$ ).

- - Nachtr. Ebda. 1904. 87 S. $8^{\circ}$.

- Hildebrand, Ed.: Das Postwesen, Berlin 1904.

- Katscher, Leop.: Das Postwesen einst und jetzt. Berlin u. Leipzig [1906].

- Ohmann, Fritz: Die Anfänge des Postwesens und die Taxis. Leipzig 1909.

- Stumm-Halberg, Frbr. Carl Ferd, von: Das Postwesen des Deutschen Reiches 1880-1891. Berlin 1910.

Post. und Telegraphenwesen. Aschenborn, O.: Das Gesetz über das Postwesen des Deutschen Reiches vom 28. Oktober 1871 u. die Vorschriften der Reichsverfassung über das Post- und Telegraphenwesen. Art. 48-52. Berlin 1908.

- Die Entwickelung des Post- u. Telegraphenwesens in Grossberzogtum Baden.

- Die Entwickelung des Post- u. Telegraphen. wesens im Herzogtum Sachsen-Altenburg. Altenburg 1900.

- Hildebrandt, Ed.: Die hauptsächlichsten Mängel im Reichspost- unđ Telegraphenwesen. Berlin 1905 u. 1906.

- Hübel, P.: Deutsches Post- u. Telegraphenwesen unter besonderer Berücksichtigung der gesamt. Literatur. München 1912.

- Jung, J.: Entwickelung des deutschen Postu. T'elegraphenwesens in den letzten 25 Jahren. Leipzig 1893.

Prag (Deutscher Verein f. Briefmarkenkunde Prag) s. Kropf, H.: Die Postwertzeichen der Oesterr-ungar. Monarchie. 1902, 40.

Preis-(frundlage, Die neue 1917-1918. s. Marken- u. Ganzsachenhaus G. m. b. H. Berlin. l'reiskatalog der Postwertzeichen der russischen Landscbaftsämter. 〈Rural-Marken, Rural-Ganzsachen.) Geordnet nach dem Moens'schen Katalog. Berlin: Kosack 1912.

Preisliste für echte Briefmarken in Sätzen u. Paketen 1917. Leipzig, Härtelstr. 23: Albert Friedemann 1917. 76 S. $8^{\circ}$.

[Die früheren Preislistcn s. u. "Friedemann, Albert".]

Preissteigerung von Postwertzeichen. Schindler, A.: Über die willkürliche Preissteigerung von Postwerthzeichen, namentlich der altdeutschen. Antrag 5 auf 1. II. Deutschen Philatelistentag zu Frankfurt a. M. 1890.

I'reussen. Fouré, Georges: Üher die in Verkehr gewesenen Postmarken u. gestempelten Freischeine von Preussen. Berlin 1881.

- Kalckhoff. Franz: Die Preussischen 'l'elegraphen-Harken. Berlin 1898.

- Obrt, Paul: Preussen. Leipzig 1896.

[Krötzsch, Handb. Bd. 13.]

Priebatsch, Leopold: Uehersicht aller bekannten von 1840 bis August 1863 ausgegebenen Franco-Alarken (Timbres-PostePostage Stamps), welche zu den beigesetzten Preisen zu haben sind durch L. P. Breslau: Selbstverlag 1863. $30 \mathrm{~S} .8^{\circ}\left(16^{\circ}\right)$. [3. a. "Wuttig, G." unter dem gleichoamigen Titel gedruekt.]

Prietsch, F. B.: Katalog aller seit 1818 bis April 1875 emittirten Postcouverte. Im ganzen Formate ungebraucht zu haben bei F. B. P. Leipzig: Selbstverl. [1875]. 33 S. $8^{\circ}$.

Priratposten, Deutsche, u. Privatpostwertzeichen. Dieckmann, W.: Die deutschen Privatposten. Hannover 1898.

- Frdmann, A.: Die deutschen Privat-Postwertbzeichen. Leipzig 1887.

- Glasewald, A. E.: Katalog und Mancoliste såmmtlicher bis 1887 herausgegebenen deutschen Privatpostwerthzeichen. Gössnitz 1887.

- Glasewald, A. E., O. Sattler \& Fr. Waguer: Handbuch der deutschen Privatpostwerthzeichen. Gössnitz 1889/90.

- Göpfert, R.: Staatspost und Privatpost. Dresden 1887.

- Misch, Karl: Die Privatpost-Marken von kiel 0. O. и. J. $12 \mathrm{~S} .8^{\circ}$.

- Die Privatpost.Marken von Lübeck o. O. u. J. 7 S. $8^{\circ}$.

- Radenhausen, H.: Katalog der deutschen Privatpostwerthzeichen. Mähr.-Ostrau 1888.

- Schimmelfennig, W. v.: Die bildlichen Darstellungen auf den deutschen Privatmarken; in:'Taschenb.f.Briefmarken-Sammlerpro 1889.

- Sternheim, Carl: Katalog cler deutschen Privatpost-Marken. Berlin-Schöneberg 1902 u. 1909.

- Tarif u. Beförderungs-Bestimmungen der Neuen Berliner Omniluus- und PacketfahrtActien-Gesellschaft. Gegr.1884. Berlin 1892/93.

- Verzeichniss der z. Z. bestehenden deutschen Privatpost-Unternehmen. [Gössnitz S-.A. 1890.] 
Privatposten. Wagner, F.: Die PrivatpostMarken von Grimma u. Dahlen o. O.u. J. 1 S. $8^{\circ}$.

- Die Privatpost-Wertzeichen. H. 1. ${ }_{n}$ Heidelberg". Berlin-Friedenau 1912.

I'rime, Hatter: Die deutschen SchiffsbriefEntwertungen. (Sonderabdr. a. den GermaniaBerichten Bd. 11. 1914.) Leipzig, Breslau 1915. Germania-Ring. ... S. $4^{\circ}$.

Probedrucke. 8. Essais u. Probedrucke.

Prozesse, philatelistische. s. Joseph, Dr.; Zechmeyer, G.; Stadthagen, Julius.

PriickJer, J. C.: Katalog sämmtlicher bis Anfang 1886 erschienenen österreich-ungar. Briefmarken, Couverts, Postkarten, Anweisungen, Begleitadressen, Telegraphenmarken, Nachnahmekarten etc. mit Preisangabe. Budapest: Selbstverl. 1886. 40 S. $8^{\circ}$.

Prifung, Die, von Postwertheichen u. deren Entwerthungen. Vortrag 1897. 8. Krötzsch, Hugo.

Piiscluel, Angust. Mlitarbeiter am, GroBen Handbuch der Philatelie. s. u. "Mexiko".

Quecusland. Cohn, David: Dic Postwerthzeichen von Queensland. Berlin 1881.

Rateulausen, Heinxich: Die Aufbewahrung der Briefmarken. Ein Beitrag zur Conservirungsfrage. s. "Philatelia" (Verein), in: Vereinsmittheilung der" Philatelia". Sondershausen 1887.

Katalog der deutschen Privatpostwerthzeichen. Mähr.-Ostrau: J. Kittl 1888. 33 S. $8^{\circ}$.

- Sollen wir Briefmarken in Paketen oder Sätzen kaufen? Ein Essai von H. $R$. in: Isakovics, A. v.: Internationales Tausch-, Adress- u. Hülfsbuch f. d. Tauschverkehr. T.2.

Radies, P. r.: Die k. k. Post in Krain und ihre geschichtliche Kntwickelung. Laibacb: v. Kleinmayr \& Bamberg 1896. 153 S. w. 1 Taf. $8^{\circ}$.

Raditch \& Co., (x.: Katalog über Orientalische Postwerthzeichen. Zsgest. nach Mejer's u. Moschkau's V. Haudbuclı für Postmarkensammler $u$. mit billigsten Preisen versehen per Stück, Satz, 100 Stück, 500, 1000 und 2000 Stück. Hrsg. . . von G. R. \& Cie. Constantinopel: [Selbstverl.] 1888. VlII S. $8^{\circ}$.

laritaiten. Haas, Theodor: Die Geschichte unserer groBen Raritäten. Vortrag gehalten auf dem XVI. Deutschen Philatelisten-Tag... in Leipzig (u. HaIle a.S.) 1904.

- Preisangabe von Raritäten; in: Jeran, O.: Wegweiser f. Verkehrswerthzeichen-Sammler (Philatelisten-Fibel).

Rarititentafel. s. Stock, Ernst.

Rave1, Ernst Johaun: Illustr. Katalog der Postwerthzeichen ... ron Italien und den ebemaligen italienischen Kleinstaaten ... [Selbstverl. 1894.] $42 \mathrm{~S} .8^{\circ}\left(16^{\circ}\right)$.

[A. s. "Kirch, R." unter dem gleichlautenden Titel gedruckt.]
Redwitz, Ferdinand: Gelegenheits - Offerte Nr. 1-. Stuttgart: Ferdinand Redwitz [1890-].

Vorschiedene Ausgaben in wecheeindem Format, teilweice auch u. d. 'L': , 'Preis-Liste über Briefmarken".]

Reeder, Indwig. s. Brendicke, Hans: Die Ludwig Reederschen Sammlungen zur Ge. schichte des Postwesens. Berlin 1898.

Rehfeld, 0tto. s. Kühtmann's Posthandbuch. 20. Aufl. Kriegsausg. 1916 .

Regensburg (Internationale Postwertzeichenausstellung). Katalog zur I. Internationalen Postwertzeichenausstellung zu Regensburg. Mit Spezialabteilung der Postwertzeichen des früheren fürstl. Thurn $u$. Taxisschen Postgebietes. Unter dem Protektorate Sr. Durchlaucht des Fürsten Albert von Thurn u. Taxis. Veraustaltet vom Briefmarkensammler-Verein Ratisbona (A.V.) im Reichssaale bezw. blauen Saale des Rathauses Regensburg rom 6.-11. Mai 1899. Regensburg: [Ausstellungsleitung.] 1899. $20 \mathrm{~S} .8^{0}$.

Reichenheim, Franz: Einrichtung der Markenbogen Frankreichs. s. Berlin. (Berliner Philatelisten-Klub.) in: Festschrift zur Feier des 25 jähr. Bestehens des B. Ph.-KI. Berlin 1913.

Reichspost. s. Beck, Otto; Ritter von Rittershain, Gottfried.

- s. a. Krötzsch, Hugo: Permanentes Handbuch der Postfreimarkenkunde. Bd. 1.

Reichspostamt. Die Klagen über die Feldpost. Berlin o. J. [1914] $12 \mathrm{~S} .4^{0}$.

- Weshalb hören die Klagen über die Feld. post nicht auf? Berlin 0.J. [1915] $5 \mathrm{~S} .4^{0}$.

- Merkblatt für Feldpostsendungen. Berlin 1914. Gedr, in der Reichsdruckerei. $2^{\circ}$.

- Post- u. Telegraphen-Nachrichten für den Verkehr mit den Deutschen Schutzgebieten. Hrsg. rom Reichspostamt nach dem Staude v. 1.Juli 1913. Berlin (1913) Reichsdruckerei. -.S. $8^{\circ}$.

Reichspostmuseum s. u. Berlin.

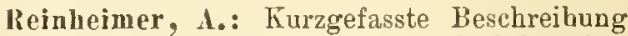
der Essays-Sammlung von Martin Schroeder, Leipzig. Zsgest. in den Jahren 1893-1902. Beschrieben von A. R., Frankfurt a. M. Leipzig: Carl Ernst Poeschel 1903. 58 S. nebst 72 Taf. mit Narkenabb. $8^{\circ}$.

- Katalog der deutschen Entwertungsarten von 1849-1875. 2 Hefte. Frankfurt a. M.: J. H. Schloss [1891-92]. $8^{\circ}$.

$$
\text { H. 1. } 1891.40 \text { S. m. } 600 \text { Abb. }
$$$$
\text { 2. 1892. } 52 \mathrm{~S}, \mathrm{~m} .700 \mathrm{Abb} \text {. }
$$

- Der bayerische Miuhliadstempel. (Sonderdruck aus „Der Philatelist" 1892.) Leipzig: 1892. 4 S. m. Abb. $8^{\circ}$.

- Illustr. Preiskatalog der deutschen postalischen Entwertungsarten. Bearb. von A. R. Mit 690 Abbildungen. Dresden: Internationaler Philatelisten-Verein 1894. $52 \mathrm{~S} .8^{\circ}$. 
Reitz Edler von Bollhein, Gustav: Das Haupt der deutschen Philatelisten (A. Moschkau). Separatdr. aus Krehn u. Loewingers Internat. Philatelisten-Adressbuch. Wien:GesellschaftsBuchdruckerei 1882. 11, 4 S. $8^{\circ}$.

- Die Pestwertzeichen ven Badeu. (Vortrag, gehalten im Wiener Philatelisten-Club.) Wien: [S. Friedl] 1880. $4 \mathrm{~S} .8^{\circ}$.

- s. a. Beschoren, Paul: Die Postwerthzeichen Sachsens.

IRensiug, lranz Josef: Geschichte des Pestwesens im Fürstbistum Münster. Hildesheim: A. Lax 1909. VIl, 88 S. m. 1 Karte. $8^{\circ}$.

(Beiträge f. d. Geschichte Niedersachsens u. Westfalens. II, 20.)

liestbestände. Glasewald, A. E.: Restbestände u. deren Schicksale. Vertrag. Gössnitz 1908.

Renterskiöld, Ixel le: Die Kantonalmarken der Schweiz u. deren Fälschungen. Bearb. von A. de R. In deutscher Sprache veröffentlicht durch die Redaction der "Pestwertzeichenkunde" u. Hans Kirchloofer. Lausanne: Kirchbofer [1890]. 39 S. u. 2 Taf. $8^{\circ}$.

Riesenlyiefwarken. s. Sammlung von 100 Riesenbriefmarken.

Riise, 0. V.: Die Postmarken von Dänemark. Eine philatelistische Studie. (Sonderabdr. aus Postwertzeichenkunde" 1893.) MIünchen: Larisch 1893. $59 \mathrm{~S} . \mathrm{m}$. Stempel-Abb. $8^{\circ}$.

Rísigari, L.: Die Marken der italienischen Kleinstaaten. Vortrag. 8. Deutscher Philatelistentag in: Stenograph. Bericht iuber die Verhandlungen des VII. D. $\mathrm{Pb}$. zu Mannheim 1895.

Ritter von Rittershain, Got1 fried: Die Reichspost der römischen Kaiser. Berlin: Carl Habel 1880. 32 S. $4^{\circ}$ (gr. $\left.8^{\circ}\right)$.

Roggenstroh, Hernann: Die Postwerthzeichen von Rumänien, Moldau, Moldau-Walachei, Fủrstentum Rumänien, Königreich Rumänien. Bearb. im Auftrage des Vereins für Briefmarkenkunde zu Nagdeburg vom Vereinsmitgliede H. R. . . . Magdeburg: [Verein f. Briefm.-Kunde 1894.] 20 S., 4 Taf. Markenabb. u. 1 Taf. Abbstempelungen. $4^{0}$ (fol.).

Rommel, 0tto: Baden. (Postfreimarken.) Leipzig: Krëtzsch 1893. $12 \mathrm{~S}$. u. 1 Taf. $8^{0}$.

(Krötzsch, Handb. Bd. 2.)

- Bayern. (Pestfreimarken.) Leipzig: Krötzsch 1893. 16 S. u. 2 Taf. $8^{\circ}$.

(Krötzsch, Handb. Bd. 3.)

- Bergedorf. 1. Aufl. Leipzig: Krötzsch 1893. 10 S. u. 1 Taf. $8^{0}$. [2. Aufl, s. u. "Krëtzsch".] (Krötzch, Handb. Bd. 4.)

- Geschichte der sächsischen Zeitungsmarke 3 Pfennige rot. (Sonderabdr. aus der lll. Briefmarkenzeitung, Januar-Mai 1894.) Leipzig: Ernst Heitmann 1894. 48 S. u. 1 Taf. $8^{\circ}$.

- Katalog der nordischen Bypostmarken s. Rommel, Otto: Die Privat-, Eisenbahn- und Dampfschiffsmarken von Skandinavien und Finnland. liommel, 0tto: Marocco, seine Post und seine Pestwertlizeichen. (Sonderdir. aus "Der. Philatelist", Juni 1906 bis Juni 1907.) "[Leipzig: Solbstverl. 1907.] 87 S. $8^{\circ}$.

- Kleinere Abarten bei den Marken für die fremdlherrlichen Postanstaiten in Marecce. (Sonderdr. aus „Der Philatelist“, März bis Juni 1908.) [Euda. 1908.] 24 S. 80.

- Die chinesischen Lokalmarken. (Sonderdr. aus Székula Bricfmarken-Verkehr, März 1906 bis Januar 1907.) [Budapest: Béla Szekula 1907.] 16 S. $8^{\circ}$.

- Die Pestwertzeichen des Bergederfer Pestbezirks. München: A. Larisch 1892. 56 S. m. Abb. $8^{\circ}$.

- - Nachtr. 1. 1892. 15 S. m. Stempelabb. $8^{\circ}$.

- - Nachtr. 2. (Sonderabdr. aus "Philatelist 1894 Nr. 8/10.) Dresden: lnt. Phil. - Verein 1894. 22 S. $8^{\circ}$.

- Nachtr. 3. (Sonderabdr. aus "Philatelist" 1895 Nr. 1/6.) Dresden: Int. Phil. - Verein 1895. $24 \mathrm{~S} .8^{\circ}$.

- Die Postwertzeichen von Lübeck. (Separatabdruck aus der "Postwertzeichen-Kunde“.) München: A. Larisch 1895. 95 S. u. 1 Taf. $8^{\circ}$.

- Die Privat-, Eisenbahn- und Dampfschiffsmarken von Skandinavien und Finnland. Gössnitz S.-A.: A.E. Glasewald 1909. 111 S. $8^{\circ}$.

[Auch u. d. T. "Katalog der nordischen Bypostmarken bekannt].

- Die chinesischen Privatpostmarken. (Sonderdruck aus dem Allgem. Anzeiger für Philatelie.) Wörishofen: Hans Schneider 1911. 54 S. m. Abb. $8^{\circ}$.

- Deutsche Reichspost (Pestfreimarken). Leipzig: Krötzsch. 14 S. u. 2 Taf. $8^{\circ}$.

(Krötzech, Haudb. Bd. 1.)

- Special-Catalog der Postwertzeichen der Deutschen Postbezirke enthaltend alle bis Fude 1895 zur Ausg. gelangten Freimarken, Nachportomarken, Dienstmarken, sewie die Briefumschläge $\mathfrak{u}$. Streifbänder mit eingeprägtem Wertstempel nebst vergl. Preisangaben auf Grund der hervorragendsten inu. ausländ. Cataloge, Handbücher u. Preislisten. Dresden: Intern. Philatelisten-Verein 1896. $121 \mathrm{~S} . \mathrm{m} . \mathrm{Abb} .8^{\circ}$.

- Studie über die Postwerthzeichen von Peru. (Sonderdr. aus der Pestwertzoichenkunde.) München: A. Larisch 1890.86 S. 80.

- Übersicht der in der Zeitungsliteratur des Jahres 1894 über die Postwertzeichen der Deutschen Staaten enthaltenen Aufsätze etc., sowie der über diese Gebiete in dem gleichen Zeitraum erschienenen Honograplien. [Leipzig o. Verlagsangabe 1895.] $41 \mathrm{~S} .8^{\circ}$. [Sonderdruck aus: „Die philatelistische Litteratur", Jg. 1 Nr. 3-10.]

— - ... des Jahres 1895 ... [Ebda. ๑. Verlagsangabe 1896.] 35 S. $8^{\circ}$. [Sonderdr. aus: "Die philatelistiscbe Litteratur", Jg. 2 Nr. 1-4.] - - . . des Jahres 1896 ... [Ebda. o. Verlagsangabe 1897.] $32 \mathrm{~S} .8^{\circ}$. [Sonderdr. aus: ,Die philatelistische Litteratur", Jg. $3 \mathrm{Nr}$. 2-9.] 
Rommel, ot10: Verzeichnis der wichtigsten philatelistischen Werke und Abhandlungen des 1n- und Auslandes mit AusschluB der Zeitungsliteratur. Zsgest. v. Dr. O. R. Chemnitz: Paul Kohl G. m. b. H. 1907. 16 S. $8^{\circ}$.

— s. a. Krötzsch, Hugo: Permanentes Handbuch der Postfreimarkenkunde.

- s. a. Zeitungs-Kiatalog, Internationaler Philatelistischer 1910 .

Ronmel, 01to \& llugo Krötusch: Braunschweig. Leipzig: Krötzsch 1893. 14 S. u. 4 Taf. $8^{\circ}$

(Krötzsch, Handb. Bu. 5.)

Bremen. (Postfreimarken.) Leipzig: Krötzsch 1893. 14 S. u. 6 Taf. $8^{\circ}$.

(Krötzech, Handb. Ed, 6.)

- Hamburg. (Postmarken.) Leipzig: Krötzsch 1893. 20 S. u. 9 Taf. $8^{\circ}$.

(Krötzsch Handb. Bd. 7.)

Rosenberg, Adulf: Die Postwertzeichen Helgolands. Vortrag. s. Deutscher Philatelistentag; in: Stenograph. Bericht ì. die Verhaudlgu. des V1I. D. Ph. zu Mannbeim 1895.

Rosenkranz, Albinus: Die Schleswig-Hol. steinische Post 1848-1852 und deren Postschillinge. Kiel (u. Leipzig: Ernst Heitmann in Komm.) 1891. 76 S. u. 10 Taf. m. Abb. $8^{\circ}$.

- Schleswig - Holsteinische Postanweisungen. s. Berlin (Berliner Philatelisten-Klub); in: Festschrift z. Feier des zehnjähr. Bestehens des B. Ph.-kl. Berlin 1898.

- Schleswig-Holstein (Postmarken). Leipzig: Krötzsch 1897. 138 S. u. 14 Taf. $8^{\circ}$.

(Krötzol, Handb. Bd. 15.)

Rotach, Arnold: Das Postwesen der Stadt St. Gallen von seinen Anfängen bis 1798. St. Gallen: Fehr 1909. 98 S. $8^{\circ}$.

Rubell, Edvard M.: Briefmarkenpreisliste Nr. 15. [Ausgabe] 1917. [Auch u. d. 'T. „Neue Europa-Preisliste*.] Berlin W. 8, Jägerstr. 5960 a. d. Friedrichstr. Selbstverl. 1917. $128 \mathrm{~S} .8^{\circ}$.

Kurze Inhaltsangabe: Altdeutgche Staaten S. 1-17. Üriges Europa S. 17-92. Vuerseeieche Staaten: Aitutaki-Jungferninseln S. 92-128.

[Die früberen Briefmarkenpreislisten erechienen in Kopenhagen in diniecher Syrache.]

- s. a. u. Maier, M. Kurt: Preisliste 1917 u. Marken-u. Ganzsachenhaus: Die neue PreisGrundlage 1917-1918, mit gleichlautendem Inhalt gedruckt.

[Ein Katalog in Form einer Zeitunge-Korrespondenz, der von jedem Händlor in beliebiger Anzahl mit oder ohne Aufdruck geiner Firma bezw. verändertem Umschlagt. beetellt werden tann und als vorlänfiger Ersatz für den oeit 191 j nicht erschienenen Senf-Katalog gelten eoll].

- Vollständige Preisliste von Dänemark, Finnland, Hamburg, lsland, Norwegen, SchleswigHolstein, Schweden u. Dänisch-Westindien. 1. u. 2. Aufl. Kopenhagen: Selbstverl. 1889.

- - 3. Aufl. 1890.

[Ausser dieseu Preiglisten ergchienen seit dem Jahre 1888 noch eine Anzahl Preislieten allgem. Inhalts, ohne besonderen literarischen Wert.]
Riibsau, Josef: Postgeschichtliche Dokumente aus dem fürstlich Thurn $\mathbf{u}$. Taxisschen Archiv. $\mathbf{1 5 0 4}$ bis $1866\langle\mathbf{1 9 0 9 \rangle}$. (Sonderabdr. aus dem Archiv für Post u. Telegraphie Nr. 11 vom Jahre 1910.) Berlin 1910. Gedruckt in der Reichsdiuckerei. $22 \mathrm{~S} .4^{\circ}$.

Riilsam, Josef \& Rudolf Freylag: Postgeschichtliche Dokumente des Fürstlich Thurn u. Taxisschen Zentralarchivs zu Regensburg $\langle 1504-1909\rangle$ auf der Internationalen Ausstellung fül Buchgewerbe und Graphik zu Leipzig 1914. Als Manuskript gedruckt. (Regeusburg, St. Emmeran: Fürstl. 'Thurn u. Taxissches Zentralarchiv August 1914.) 48 s. u. 1 Bild-Taf. $4^{\circ}$.

Riickerinuelungen; in: Moschkau, A., Zur Geschichte der Philatelie.

Kulı, J.: Beitrag zur Geschichte des Postamts Bebra. Mlarburg: N. Spiess 1892. 45 S. $8^{\circ}$.

- Beitrag zur Geschichte des Postamts Marburg von 1784-1884. Hbda. 1885. 38 S. $8^{\circ}$.

Rull, Moritz: Der Briefmarkenfreund. Eine Sammlung originalgetreuer Abbildungen der Iriefmarken aller Welttheile in vielfach vergröBertem Maassstabe. Leipzig: Moritz Rubl [1864-65]. 96 Taf. m. farb. Abb. 8*.

[Eröchienen in 12 Lfgn, zu je $8 \mathrm{Taf}$. LIg. 1 ent. halt ein Vorwort, const ohne jeden Text. Vom Jahre $1872 \mathrm{ab}$ wurden dann die Restbetiände der Tafeln u. d. T.: "Sammlung von 100 Rieseu-Briefmarken in Orig. Farben" veräussert. Siehe auch u. "Sammlung etc. ${ }^{\prime} j$

Rumänien. Duerst, Georg B.: Die Essais von Kumänien. Berlin 1913 .

- Ohrt, Paul: Mloldau 27 parale. [Charlottenburg 1900.]

- Die 27 Parale der Moldau (Fälschung); in: Moschkau, A., Zur Geschichte der Philatelie.

- Roggenstroh, H.: Die Postwerthzeichen von Rumänien, Moldau usw. Magdeburg [1894].

- Steinberg, L.: Preis-courant ïber Briefmarken von Rumänien. Bucarest [1895].

- Wassermann, O. u. Heiurich Fränkel: Rum̈̈nien, Ausgaben 1866-1872. Berlin 1898.

liund um Berlin. s. Vereinsgeschichte des Philatelisten - Vereins "Rund um Berlin" 1901-1905.

Rıral-Marken u. Ganzsaclen. Preiskatalog der Postwertzeichen der russischen Land. schaftsämter. (Rural-Marken, Rural-Ganzsachen.) Berlin 1912.

- Schmidt, C. \& A. Fabergè: Die Postwertzeichen der russischen Landschaftsämter. St. Petersburg 1909-

Rural-Posten. s. Lübkert, Hugo.

kussland. Baggo, E. v.: Die coursirenden Ganzsachen von Pussland nebst Preisverzeichniss. Dorpat 1889-90.

- Bochmann, Eugen von: Die Postmarken des russischen Rieiches u. deren Entwertung. Leipzig 1892.

- Die Postmarken des russischen Kaiserreiches. Leipzig 1895. 
Russland. Hruby, R.: Katalog aller türkischen, ostrumelischen, bulgarischen und russischen Levantepostmarken, Karten u. Couverts mit Preisen. Constantinopel 1884-1891.

-- Jürgens, Woldemar: Katalog-Preisliste (Nr. 5-8) aller Marken, Karten und Briefumschläge von Finnland u. Russland. Helsingfors $1880-83$ u. Reval 1886 .

- Lager-Verzeichniss (Nr. 9). Specialitait: Russland. Reval 1891.

- Kohl, Paul: Illustr. Katalog der Freimarken von Europa. Abt. 4. "RuBland." Chemnitz $1894-95$.

- Die Lokalpostmarken KuBlands; in: Moschkau, A., Zur Geschichte der Philatelie.

- Litbkert, Hugo: Handbuch aller bis 188I bekannt gewordenen Postwerthzeichen der Rural-Posten in Fussland. Wien 1882.

- Preiskatalog der Postwertzeichen der russischen Landschaftsämter. Berlin 1912.

- Schmiat, C. 11. A. Fabergé: Die Postwerthzeichen der russischen Landschaftsänter. St. Petersburg $\mathbf{I} 909$.

- s. a. u. Rural-Marken u. Ganzsachen, RuralPosten u. Wenden".

Saehs, Franz: Die Dienstganzsachen Nenseclands. s. Berlin (Berliner Philatelistenklub); in: Festschrift z. Feier des 25 jähr. Bestehens des B. Ph.-Kl. Berlin 1913.

- - Fin Vergleich der Preise der massgeben. den Kataloge, s. Berlin (Berliner Philatelisten-Klub); in: Festschrift zur Feier des 25 jähr. Bestehens des B. Ph.KI. Berlin 1913.

Saehse, Paul: Was dann? s. Berlin (Berliner Philatelisten-Klub); in: Festschrift z. Feier des zehnjähr. Bestehens des $\mathrm{B} . \mathrm{Ph}$.-Kl. Berlin 1898 .

Sachsen. Beschoren, Paul: Die Postwerthzeichen Sachsens. Wien [1881].

- Hüttner, G. F.: Das Briefpostwesen des Königlich Sächsischeu Postbezirks iu soiner neuesten veränderten Einrichtung. Unentbehrliches Taschenbuch für das correspondirende Publikum. Leipzig: Gustav Brauns 1851. XXIV, 88 S. $8^{0}$.

- Kloss, Paul: Geschichte der Post-Werthzeichen des Königreichs Sachsen. Leipzig (1882).

- Krebs, Kurt: Das kursächsische Postweseu zur Zeit der Oberpostmeister Johann Jakob Kees I u. II. Iseipzig 1914.

- Krötzsch, Hugo: Hirschfeld'sche Entwürfe u. Druckproben zu den Sächsischen Postfreimarken. Berlin 1898.

- Lindenberg, Carl: Die Briefumscbläge von Sachsen. Berlin 1894.

- Rommel, Otto: Geschichte der sächsischen Zeitungsmarke 3 Pfennige rot. Leipzig 1894.

- Schaefer, Gustar: Geschichte des sächsischen Postwesens. Dresden 1879.
Sacheu-Alteuluurg. Die Fntwickelung des Post- und Telegraphenwesens im Herzogthum Sachsen-Altenhurg. Altenburg S.:A. 1900.

Samoa. Kohl, Paul: lllustr. FreimarkenKatalog "Englische Kolonien" Abt. 3 ... Samoa... Chemuitz 1894-95.

Sammlung von 100 Riesen-Briefmarken in Original - Farben. Leipzig: Mloritz kubl [1872]. I00 farb. Tafeln. $8^{0}$.

[8. a. Kuhl, Moritz: Der Briefmarkenfreund.]

Sill Marino. Kohl, Paul: Illustr. Katalog der Freimarken von Europa. Abt. 7. „San Mlarino." Chemnitz 1894-95.

Sandknhl, f. s. Deutscher Philatelistentag. Bericht ü. d. a. 18. August 1889 zu Mainz... abgehaltenen Ersten Deutschen Philatelistentag.

Sardinien. Die Couverte Sardiniens u. ihre Nachahmungen; in: Moschkau, A., Zur Geschichte der Philatelie.

- Sardinien 3 Lire bronce (Fälschung); in: Moschkau, A., Zur Geschichte der Philatelie.

Sartori, freorg: Catalog aller seit 1840 bis Ende 1872 ausgegebenen Briefmarken. Mit beigesetzten Verkaufspreisen. Frankfurt a. MI.: [Selbstrer]ag 1873]. 50 S. $8^{\circ}$.

Sattler, 0. s. Glasewald, A. E., O. Sattler \& Fr. Wagner: Handbucl der deutschen PrivatPostwerthzeichen.

Saner, Karl C1.: Permanenter PostwerthzeichenKatalog. (Beilage zum Sauer'schen Permanentalbum.) Mährisch-Ostrau: [Kittl 1887]. 40 S. $8^{\circ}$.

[Nur bis "Brasilien" erschienen.]

- Permanenter Briefmarken-Katalog. Belgien und Congo-Staat. Ebda. [1889]. I6 S. $8^{0}$.

- - Niederland und seine Colonien. Ebda. [1889]. 20 S. $8^{\circ}$.

[Die Teile: Dentschland, Portugal u, seine Colonien, sowie Österreich-Ungarn" g. u. Glasewald, A. E.; Gutmensch, J.u. Neulinger, E. v. unter gleichnamigom Hauptitel gedr.]

Selhaefer, Gustar: Geschichte des sächsischen Postwesens rom Ursprunge bis zum Übergang in die Verwaltung des Norddeutschen Bundes. (Mit dem Bildnis des Churfürsten August u. 10 anderen Abb.) Nach archivalischen Queilen hearb. von G. S. Dresden: గ. v. Zahn 1879. 248 S. 8 .

Schïfer, J.: J. Sch's Scliwarzes Buch. Verzeichuis jener Personen, welche ihren Verpflichtungen nicht nachkommen nebst Angabe aller grösseren empfehlenswerten Vereine, Börsen, IIuseen, Postwertzeichen-Besorgungs- u. Prüfungsstellen. Hrsg. v. J. Sch. Turn Mlagurelle, Rumänien, Craiova: [Selbstverl.] 1897. $72 \mathrm{~S} .8^{0}$.

Sehardey, Panl: Wohin soll das führen? Vortrag. s. Deutscher Philatelistentag; in: Stenograph. Bericht ü. d. XVIIl. Deutschen Philatelistentag . . . in Nüruberg 1906. 
Seluarli, Friclrich: Die Ansprüche u. Rechte der fürstl. Thurn u. Taxis'scheu Post gegenüber den Eisenbahn-Unternehmungen, mit besonderer Beriicksichtigung der TaunusEisenbahn. Frankfurt a./Main 1840. $4^{\circ}$ (gr. $\left.8^{\circ}\right)$.

Scluabek, Gustav: Katalog aller seit dem Tahre 1840 bis auf die neueste Zeit ausgegebenen Brief- und Couvert-Marken. Nach der Alfred MLschkau'schen Sammlung bearb. und hrsg. von G.S. Leipzig: Exped. der Deutschen Briefwarken-Zcitung 1871.96 $\mathrm{S} .8^{\circ}$.

- Katalog aller bekannten bis auf die neueste Zeit ausgegebenen Briefmarken (Post-Freimarken - Couverts - Streifbänder - Karten). Bearb. von (1. S. Leipzig: Ed. Wartig 1874. 134 S. $8^{\circ}$

[Umschlagt, teilweise mit dem Zusatz: nZwoite vollständig umgearb. Aufl" versehen.]

- - Nachtr. 1 zur zweiten vollstäudig umgrearb. Aufl. bis November 1874. Ebda. 1875. $18 \mathrm{~S} .8^{\circ}$

- s. a. Dauth, H. J.: Manco-Listen f. Briefmarkensammler 1884; Pilzecker., O.: Preisbuch Nr. 1 [1889]; Senf, Gebrüder: Manco. Liste für Briefmarkeusamm]er [1887 - 1892].

Schaufuss \& Stolpe: Wegweiser für Marken. sammler. Leipzig: Schaufuss \& Stolpe 1911 bis 1914 ; je $64 \mathrm{~S} .8^{\circ}$.

Sehaupmeier, Charles: Neuer Katalog der Postwertzeichen Frankreiclis u. seinerKolonien von 1849-1893. Alle Aufdrucke ausgeschlossen. Darmstadt: E. Schaupmeier [1893]. 28 S. $8^{0}$.

- Neuer Katalog der Post-Wertzeichen der französischen Kolonien. Darmstadt: E.Schaupmeier. Juni 1893. $8 \mathrm{~S} .8^{\circ}$.

Scheihe, Max: Mittel und Wege, die Popularität des Briefmarkensammelns im Publikum zu förderu. Vortrag. s. Deutscher Philatelistentag; in: Stenograph. Bericht des XXIII. Deutschen Philatelistentages . . . zu Wien 1911.

Sehell, Vittinghofr-, Frhr. vou: Mitarbeiter am GroBen Handbuch der Pliilatelie". s. a. unter ${ }_{n}$ Vittinghoff-Schell, Frhr. von (der gleiche Verfasser).

Selidlof, Bertholl: Was muB der Briefmarkensanmler wissen? Ein Leitfaden zum Anlegen einer Briefmarkensammlung. Berlin: Hugo Steinitz 1904. 76 S. $8^{\circ}$.

Schiffspost, Die, auf dem Bodensco. s. Maus, J.

Schitfs- wul Marinepoststempel-Sammlung, Deutsche. s. Knopf, O.; Priwe, Walter; Schmidt(-Schwerin), Max.

Schilliug, C. F.: Katalog aller seit 1840 bis auf die neueste Zeit ausgegebenen Briefmarken, welche zu den verzeichneten Preisen durch C. E. Sch. zu beziehen sind. Leip. zig: Selistverl. 1876. 55 S. $8^{\circ}$.

Schiumelfennig, W. V.: Die bildlichen Darstellungen auf den deutschen Privatmarken; in: Taschenbuch für Briefmarken-Sammler pro 1889.
Schindler, August: Gründung eines Centralorganes für die Gesammat-Interessen der Briefmarkenkunde. s. Deutscher Philatelistentag. Antrag 3; in: Bericht über den II. Deutschen Philatelisten-Tag zu Frankfurt a./M. 1890.

- Über die willkürliche Preissteigerung von Postwerthzeichen, namentlich der altdeut schen. s. Deutscher Philatelistentag, Antrag 5; in: Bericht über den II. Deutschen Philatelisten-Tag zu Frankfurt a./M. 1890.

Schleswig-Holstein. Rosenkranz, A.: Die Schleswig-Holsteinische Post 1848-1852 u. deren Postschillinge. Kiel 1891.

- Die Postfreimarken der Herzogtümer Schleswig-Holstein. Leipzig 1897.

(Krölzscb, Handb. Bd. 15.)

- Schleswig-Holsteinische Postanweisungen 1865-1867. Berlin 1898.

- Ruben, M. H.: Vollständige Preisliste von .. Schleswig-Holstein ... Kopenhagen $1889 / 90$.

Selunidt, Gustar: Tauschrerkehrbuch für Postwertzeichensammler. Leipzig: Verlag des Universal - Briefmarken - Albums (Gustav Schmidt) 1903. 104 S. 4\%.

Schmidt (-Schwerin), Max: Verzeichnis der Kaiserl. Deutschen Marine-Schiffsposten. (Beil. zur Mitteldeutschen Philatelisten - Zeitung Jg. $1904 \mathrm{Nr}$. 6-11). Gössuitz S.-A.: A. E. Glasewald 1904.18 S. $8^{\circ}$.

Schmilt, Rudolf: Dr. Alfred Moschkau. Eine biographische Skizze. Mit Portrait. Als Manuscript gedr. Leipzig: Louis Senf 1877. 16 S. $8^{\circ}$.

Schmidt, S.: líatalog u. Preisverzeichnis aller existierenden Briefmarken. Erste Aut. Kopenhagen: Selbstverl. 1880. $88 \mathrm{~S} .8^{\circ}$.

Schmidt, C. E A. Fabergé: Die Postwertzeichen der russischen Landschaftsämter. Lfg. 1-10. St. Petersburg: Sektion des Intern. Philatelisten-Vereins "Dresden". 1909. 410 S. u. 52 Taf. m. Abb. gr. $4^{\circ}$.

[Unvollendet.]

Schuickert, Generalpostdirektor, Biographie. s. Müller, Hans v.

Sclınejder, Frauz: Katalog der Bayerischen Postkarten nebst Preisverzeichnis. (Durchges. Sondertruck a. d. "Ganzsachensammler" 1912/15.) Berlin: Philipp Kosack \& Co. 1915. 35 S. m. Abb. $4^{\circ}$.

Selıoltze, Karl Johannes [Pseud.: Kar] Johannes]: Praktische Anleitung zum Briefmarkensammeln. Leipzig: Johannes Scholtze [1896]. 99 S. $8^{0}$.

- 2. Aufl. Berlin: S. Mode 1900. 105 S. 80. [s, a. Johannes Carl.]

Schrauka, Eduard Maria: Datenzeiger der Welt postgeschichte. Leipzig : Slavische Buclih. 1893. 212 S. u. 4 Bilder-Tat. $8^{\circ}$.

- Das Gelbbuch (Bertels Postbüchel). Prag: J R. Vilcmek 18 .?? S. $8^{\circ}$. 
Schreiber, I. J.: Staltbripfbeförderung für 2 Pfennig. Juli 1874. .1. J. Sehreibers Briefu. Druckschriften-Expedition "Berlin". Berlin: Selbstverl. 1874. 15 s. $8^{0}\left(16^{\circ}\right)$.

schroeder, Martin s. Keinheimer, A.: Kur\%gefasste Beschreibung der Essays. Sammlung von Martin Schroeder, Leipzig. Frankfurt a./M. 1903.

- s. a. Thier, Max: Kurzgef. Besprechung der Briefmarken-Sammlungen von Martin Schroeder, leipzig. Charlottenburg 1903.

Schroeder, Oswald: Briefmarken-Catalog. Leiprig: Selbstverlag 1886. $30 \mathrm{~S} .4^{n}$.

Sichröter, Carl: Der Weltpostverein. Bern: K. J. Wyss 1900. 34 S. m. 3 Taf. 8".

sehuhert, (i. W.: Der Fïhrer im Labyrinthe ler bisher erschienenen Briefmarken, etc., aller Länder, oder specielles Verzeichniss der auf den Briefmarken u. Converts vorkommenden Sinnbilder, Portraits u. anderer Gebilde, Allegorien, Wappen, (verientschter) fremdländischer Werthbezeichnungen, der auf Mar. ken repräsentirten Landeswappen, ingleichen der vom Jahre 1865 ab bis mit 1866 neu emittirten Mlarken, etc., nebst anderen einschlagenden Notizen, behufs leichterer Erkennung und sicherer Einschaltung derselben in die Mlarkensammlungen; zsgest. von G. II. Sch. Dresden: Burdach'sche Hofbuch handls. 1867. $48 \mathrm{~S} .8^{\circ}$.

Schiller, Friedrich: Die persischẻ Post u. die Postwertzeichen von Persien u. Bucbara. Wien: Sigmund Friedl u. E. Heim 1893. 90 S. u.

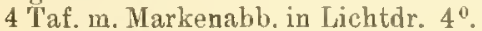

[Ausserdem hierzu 4 Nachtr.-Seiten ohne Zähluug.]

Schultze, Joh. 0.: Preisbuch der Marken von Europa. 1.-11. Auf. Oelsnitz i./Frzgel.: Jol. O. Schultze $1890-1904.11$. Aufl. 1904. 60 S. $8^{\circ}$

[0. a. nGlanewald, Arthur Ernst* u. , Küuast, W." unter dem gleichnamigen Titel gedrucht.]

Schulze, Alolf: Biographie; in: Jeran, O.: Wegweiser für Verkehrswertzeichen-Sammler.

- Die Schweizer Cantonal-Marken. (Als Beigabe zur Photographie.) Zürich: Selbstverl. November 1879. 4 S. $4^{\circ}$

[Hektographiert.]

Schutzblatt (Nr. 1) des Germania-Ring. Velband deutscher Postwertzeichensammlerveroine E. V. Wiu Entwurf von Dr. Pirl, Charlottenburg 5. (Bpil. zu Nr. 7 der GermaniaBerichte v. 10.7. 1912.) Essen a. d. Ruhr: Germania-Ring 1912. 4 S. $4^{\circ}$.

- (Nr. 2) des Germania-Ring usw. Die rom Germania-Ring 1912 angenommenen, Leitsätze zur Bewertung der Abstempelung ", angewandt auf die Entwertung der deutschen Schutzgebiete und Auslandsposten. Fin Entwurf von Dr. Pirl. (Beil. zu Nr. 3 u. 10 des Germania-Berichte 1913.) Ebda. 1913. 5 S. 4". (e. a. u. Pirl, Paul.)

Sehutzgebiete, Dentselse. s. Dentsche Kolonien.

Ton, Handhreh.
Sicliwanelicrger, Hugo: Uber ganze Bogen. Vortrag s. Deutscher Philatelisten-Tag; in stenograph. Bericht über d. XV1. Deutschen Philatelisten-Tag ... in Leipzig (u. IIalle a. S.) 1904.

s. a. Dauth, H. J.: Mankolisten für Briefmarken-Sammler 1884;

- Heitmanns Illustr. Handbuch 1894/95;

- Maukoliste zu Schwanebergers Briefmarkensammelbuch 1895;

- Moschkau, Alfred: Briefmarkeu-Zeitung für die Jugend [1889];

- Pilzecker, O.: Preisbuch $\mathrm{Nr} .1$ und 2 [1889 и. 1891].

Nehwarze Buch, Das, s. Lietzow, Paul.

Liste, Die, des Vertraulichen Korrespondenz-Blattes philatelistischer Vereine. Zusammenstellung der Warnungen aus den Jahren 1891 bis 1906. Hamburg: Bund Deutsch-Öster. Philatelisten-Vereine. 1907. 23 S. $8^{\circ}$.

[Urspringlich fortsetzungsweise im , Vertraul. Kor. respondenz-Blatt philatelistischer Vereine ${ }^{4}$ Juli-Dez. 1907 reröfentlicht. Frübere Erscheinung nur bis 1899 8. u. d. T. Liste, Die schwarze $\theta^{4}$.]

- s. a. Paul, Emil: Die schwarzen Listen, Kataloge und Bücher auf dem Gebiete del Philatelie.

Schwarzer Permanent-Katalog. s. GermauiaRing.

Schwalz-Sammlung. Stückweise Versteigerung s. Auktionskataloge. Koehler, Heinrich: Katalog der Briefmarken-Auktion Nr. 10.

Neliweden. Andresen, Ferd. jr.: Preisliste über skandinavische Briefmarken. Christiania 1897.

_- Djurling, Hilmer u. Rudolf Krasemann: Die Postmarken von Schweden 1S55-1905. Teipzig: Erötzsch 1908.

- Verzeichnis über die Postwertzeichen von Schweden. Stockbolm 1906, 1907 u. 1911. s. a. u. "Skandinavien".

- Koht, Paul: Mllustr. Katalog der Freimarken von Europa. Abt. 4. "Schweden." Chemnitz 1894-95.

Krötzsch, Hugo: Permanentes Beibuch mit Lichtdruck-Tafeln. T.2. "Scbweden." Leipzig 1908.

- Romme1, Otto: Die Prirat-, Eisenbahn- und Dampfschiffsmarken von Skandinavien und finnland. Gössnitz 1909.

- Ruben, M. E.: Vollständige Preisliste vou . Schweden . . Kopenhagen 1889/90.

Sveriges Filatelist Förening: Verzeichnis der Postwertzeichen von Schweden. Stockholm 1906 u. 1911.

- - Ganzsachen. Stockholin 1907.

Schwefelmeier's zeitgemäBe Betrachtungen über die Farbebezeichnungen in der philatelistischen Literatur. s. Gerdt, A.

schweiger-Lerchenfeld, 1. Hrhw, von: Das neue Buch von der Weltpost. Geschichte, Organisation u. Technik des Postwesens von 
den ïltesten Zeiten his aul die Gegenwart. Nit 99 Vollbildern, 633 Abl. im l'exte und 4 Karten. Wien u. Leipzig: A. llartleben [I892]. $952 \mathrm{~S} .4^{\prime \prime}$ (gr. 8").

[Iabalt u. a.: Die Taubenpust. - Die Falloupost S. 289-B18; Zur Geschichte des lineles. - Die Fint. wickelung des Briefrostverkelors, - Die Briefinarkeu. - Die Postkarte. - Allgemeineg uber Ganzacheu u. Philatelie S. $35:-480$.

Schwciz, biaseler Täubchen (Fälschungen); in: Moschkau, A., Zur Geschichte der Philatelie.

- l'ochmann, Eugen von: Die Schweizer Marken der Emission 1854-1863. Berlin /1898.

- Deyhle, liobert \& Cie.: Preisliste über schweizerische l'ostwertzeichen der Briefmarkenhandlung $R$. Deyhle \& Gie. Bern 1888.

- Mirsewald, C. von: Die Schweizer Marken von 1843 bis 1854 . München 1893 .

- Jiggli-IVeber, H: Über die geschichtliche Hntwicklung des Verbancles schweizer. Philat.elisten-Vereine bei Aulass seines 25 jährigen Bestehens. (Sonderdr. aus der, Schweizer Briefmarken-Zeitung $1916 \mathrm{Nr}$. 10.) Winterthur (Schweiz): Selbstrerl. 10 S. $8^{0}$.

hohl, Paul: Illustr. liatalog der Freimarken von Furopa. Abt. 6. Sclnweiz. Chemnitz $1894-95$.

- Leman, Ed. von: Spezialkatalog der P'ost. wertzeichen dej Schweiz. Lausanne 1889 u. Gent 1894 .

- Lienhard (Pfarrer): Die Entwerthungsarten ler schweizerischen Briefmarken 1843-1863. Ziiriclı 1896.

- Maus, J.: Die Schiffspost auf dem bodensee. Dresden 1899.

Mirabaud, Paul \& A. de Reuterskiöld: Die Schweizerischen Postmarken 1S13-1862. Paris 1899.

Neulinger, Eduard von: Permanenter Briefmarken-Katalog "Schweiz". Mälr.-Ostrau [1888].

- Pfenninger, O.: Handbuch der Schweizer Postwertlizejchen. Lausanne 1890.

- Kantonale und schweizerische Postentwerthungsstempel aus den Jahren 1850 bis 1854. Lansanne [1891].

lieuterskiold, Axel de: Die Kantonalmarken der Schweiz u. deren Fälschungen. Lausanne 1890 .

- Rotacir, A.: Das Postwesen der Stadt St. Ciallen von seinen Anfüngen bis 1798. St. Gallen 1909.

Sauer, C. Cl: l'ermanenter Hriefmarken Katalog. "Schweiz." s. Neulinger, Eduard von. Schulze, Adolf: Die Scliweizer CantonalMarken. Zürich 1879

— Stĩger, A.: Die eidgenössische Post. Bern 1911. - Stäger, Toh. Ant.: Das schweizerische Postwesen zur Zeit der Helvetik. Bern 1879.

- ritucki, A.: Grundriss der Postgeschichte $m$. besonderer Berücksichtigung der schweizerisehen Verkehrsverhältnisse. liern 1909.
Sehweiz. Zumstein, Ernst: Spezial-Katalog u. Ilandbuch über die Briefmarken der Schweiz. Bern 1909.

Zürich-Fälschungan; in: Moschkau, A., Zur Geschichte der J'hilatelie.

Neelig, G.: Preisbuch [der Marken] von Furopa. 1.-11. Aufl. Stettin, 1890-1904.

[s. a. u. Glasewald, Kinast, Schultze, Joh. 1]. uuter gleichnanigen Titel, wur wit geändertem Umschl. gedr.)

Sellsehopl, Wilhelm: I lie Wxpressgesellschaften des westlichen Nordamerika und ihre Wertzeichen. Vortrag anlässlich des XIX. Deutschen Philatelistentages in Hamburg gehalten. (Sonderabdr. a. d. Berliner Briefmarken-Zeitung*.) Berlin: Jhilipp Kosack 1907. 8S. $4^{0}$

- (Sonderabdr. a. "Der Philatelist" 1907, Nr.9 u. 10). Dresden: Intern, Pliilatel.-Verein 1907. $7 \mathrm{~S}, 80$

- (Separatabrug aus , lllustr. BriefmarkenIournal 1908, Nr. 2-4.) l,eipzig: Gelriider Senf 1908. 12S. 80.

- Gebraucht und ungebraucht. Vortrag. s. Deutscher Philatelistentag; in: Stenograpl. Bericht uber den XIII. Dentschen Philatelisten-Tag. Berhin 1901.

[Philalelistische Streiflichte I.]

- Postwertzeichen, Neudruck und Sonderauscrabe. Vortrag, geh, am V111. Internationalen Briefmarken-Händlertage in Berlin am 28. Jinuar 1909. Hamburg: Sellistrerl. 1909. 12 S. 80.

[Uhilatelistische Streiflichter II.]

- Preisliste über Ganzsachen. Ganze PostKarten, Doppelkaten, Kartenbriefe, Briefumschläge, Streifbänder, etc. etc., der Briefmarkenhandlung W. S. Hamburg: Selbstrerl. 1904. $95 \mathrm{~S} .8^{\circ}\left(16^{\circ}\right)$.

- -. Nr. 2 (Nachtr.). 32 S. $8^{0}\left(16^{\circ}\right)$.

- - Nr. 3 u. 4 (kleinere Nachtr.). 2 Bl. $8^{0}\left(16^{\circ}\right)$.

$\div-\mathrm{Nr}$ 5. 1910.152 S. $8^{\circ}\left(16^{\circ}\right)$.

- Nr.6. 1911. (Nachtr.) $36 \mathrm{~S} .8^{\circ}\left(16^{\circ}\right)$.

- Seltenlseiten-Buchführung. Vortrag, gelı. am iV. Internationalen Briefmarken-Händlertag am 22. Januar 1916 in Berlin. Hamburg: Selbstverl. 1916. 10 S. $8^{\circ}$.

- Western Franks, Freicouverts der American lixpress-Compagnien. Vortrag. s. Deutscher Philatelistentag; in: Stenograph. Bericht des XIX. Deutschen Philatelisten-Tages ... zu Hamburg 1907.

Nohin treiben wir"? Hine Abhandlung über Abstempelung. Vortrag, geh. a.d. Xll. lntermationalen Briefmarken.Händler-Tag zu BerIin am 24. Januar 1913. Berlin: Sellustverl. des Internationalen Postwertzeichen-HändlerTereins 1913. I5 S. $8^{\circ}$.

[Philatelistische Streiftichter III.]

Hiervon gibt es auch eine Augg. mit dem verinderten Untertitel: Ein Beitrag zur Abstempelungs. frage" und obne den Aufdruck: "Philatel. Streiflichter III". Text sonst unverindert.]

teltenheiten u, deren Preise. s. Lietzow, Paul. 
Senf, Gebriider: Die Ausbilfswarken von Tsingtau und ihre Fitlschungen. s. Frielemanu, Albert.

- Philatelistische Bibliothek. s. Bibliothel, Philatelistische.

- Fliegende Blätter aus Senf's Illustriertes Briefmarken-Journal. Zugeeignet von (i. S. Ebda. 1888 . $32 \mathrm{~S} .8^{\circ}$.

- Fliegonde Blätter für Freunde der Postwertzeichen-Kunde. Zugreeignet von G. S. Leipzig: Ebda. 1891. $64 \mathrm{~s} .8^{\circ}$.

- - [Neue Ausg.] Ebda. [1893.] $72 \mathrm{~S} .8^{0}$.

- - [Neue Ausg.] Ebda. 1899. 96 S. $8^{\circ}$.

[Enthillt u. a. Nachtrige u. text]. Buriclitigungen zu Senfs Postwertzeichen-Katalog 1898.]

Farben-Karte. (Sonderbeil. zu Senfs Illustr. Briefmarken-Journal 1888-1890.) Leipzig: Gebrüder Senf 1890. 18 'af. i. Nappe. $8^{0}$.

G. S.s Briefmarken-Journal Kalender. Angebot- u. Lebr-Büchlein für PostwertzeichenSammler. (Neue Folge der „Fliegenden Blaitter fiir Freunde der Postwertzeichenkunde*.) Mit zallreichen Abb. Hbda. 1895. 128 S. $8^{\circ}$.

- Fehlliste für Briefmarkensammler. s. v. Sent, Gebrüder: Mancoliste.

Flugblatt ïber den Sent-Katalog 1903 mit Aufforderung zor allgemeinen Abstimmung. Beigefügt ein kleiner Nachtrag zum SenfKatalog nod zur Satz-Preisliste. Ebda. 1908. 24 S. $8^{\circ}$.

- [Nene Ansg.] Hibda. 1908. 36 S. 8\%.

- G. S.'s Illustrierter Kriegsmarken-Katalog über die Postwertzeichen des Weltkriegs. Enthaltend alle seit Kriegsbeginn erschienenen in Beziehung zum Weltkriege stehenden Postwertzeichen der Länder der Mittelwächte und ibrer Verbündeten mit Preisen, sowic die Postwertzeichen des nentralen $\mathrm{u}$. feindlichen Auslandes 〈olne Preise). Mlit zahlreichen verkleinerten Abb. Leipzig: Gebr. Senf [1917]. $96 \mathrm{~S} .8^{\circ}$.

- Leitfaden der Brietuarken-Kunde für anrehende Postwertzeichen-Sammler. Hrste u. zweite Auf. Kbda. 1891. Je 63 S. $8^{\circ}$.

- Dritte Autl. Ebula. 1896. 64 S. $8^{\circ}$.

- Vierte Auf. Ebda. o. J. [1911]. 52 S. $8^{0}$.

- Manco-Liste für Briefmarken-Sammler. Bearb. auf Grund der 8. Aufl. von Schaubek's Briefmarken-Album. Iibda. [1887]. IV, $122 \mathrm{~S}$. $8^{\circ}$ [Taschenformat]. Biegsam Leinw. geb.

- auf Grund der. 9. Autl, von Schaubek's Briefmarken-Album. Ebda. [1888]. $122 \mathrm{~S} .8^{\circ}$.

- - auf Grund der 10. Aufl. von Schaubek's Briefmarken-Album. Ebda. [1889]. I20 S. $8^{\circ}$.

- Fehlliste für Briefmarkensammler. Bearb. auf Grund der 11. Aufl. von Schaubek's Brietmarken-Album. Fbula. [1890]. 121 S. $8^{\circ}$.

- Senf's Fehlliste für Briefmarken-Sammler. Bearb, auf Gruud der 13. Autl. v. Schaubek's Briefmarken-Album. Kuda. [1891]. 123 S. $8^{\circ}$.

- Bearb. auf Grund der 14. Aufl. v. Schaubek's Briefmarken-Album. Ehda. [1892]. 127 S. $8^{\circ}$.
Senf, ficbrïder: Die Marken dere dentschen Kolonien und Auslandspostimter. Auszum aus dem Senf-líatalog 1915 u. als Anluanor die Zustellungsmarken der Stadtpost Warschau. Nach den letzten Forschungen nenbearb. mit nenesten Pleisnotiernngen. [libla. 1916.] $25 \mathrm{~S} .80$

[Linseitig bed". in Format dos simf-liateloges zum Eintügen in diesen.]

- - [Neuausg.] wit allerneuesten Preinnotierungen. [Ebda. 1917.] $24 \mathrm{~S} .8^{\circ}$.

[' $Z$ weiseitig bedr.]

- Nummernliste der im 1904 er Senl-katalog autgenommenen Postwortzeichen ... Fehiliste. Wuda. 1904.157 \&. $8^{\circ}\left(16^{\circ}\right)$.

- 1905 er Senf-Katalog aufgenommenen Postwertzeichen... Fehlliste. Elula. 1905. 159 S. $8^{\circ}\left(16^{\circ}\right)$.

G. S.'s illustrierter I'ostwertzeichen-liatalor 1892. Hinthaltend simtliche bis gegen Ende 189 I erschienenen Postmarken, postalisch gebrauchte Stempelmarken, Briefumschläge, Postkarten, Kartenbriefos Postanweisungen, Streifbänder, Paket-Begteitkarten u. Rückscheine, einschlieblich lielıldıcke, Typen u Veudrucke, untar Berücksiclitigung aller Verschiedenlieiten bezügl. Wasserzeichen, Ziih nungen, Klappenstewpel, Forwate, Gummie lungen usw. Harken u. Ganzsaelien. Leipzig Gebrüder Senf (1892). XII, 592 S. mit Abu. 8". H'inband rot.]

- Zweiter Aburuck mit I'reisänderungen in Februar 1892. luda. 544 S. $8^{\circ}$.

[Hiervon gibt es auch eine Ausg. mit eingelegten leeren Blättern in rotom Einbund mit Goldtitel ]

- 1893. Marken und Ganzsachen. Hbda. (1893). XV1, 660 S.m. Abu. 8*. [Linband blau.] — - 1893. Neue Ausg.] Wbdil. (1893). $676 \mathrm{~S}$. m. Abb. 8\%. EEinb. blau.

- 1894. Marken und Giazsachen. Muda. (I894). XVI, 720 S. m. Abl. 8". [Einb. braun.] [Hiervon gibt es auch eine Ansg. mit $678 \mathrm{~s}$. in frauem Einb unter dem Namen: "Sigmund Friedt" fil $1 V^{\circ}$ en als Herausgeber.]

- 1895. Marken und Ganzsachen. Ebda. (1895). XXI1I, 83น S. m. Abb. 8\%. [Einb. grün.]

- 1896. Marken und Ganzsachen. Euda. (1896). XXVIl, $576+$ VIl, 286 S. m. Abl. 8". [Hinband grau.]

- - 1897. Marken und Ganzsachen. Hbda. (1897). XXIV, $640+$ V111,302 S. m. Abl. $8^{\circ}$. [limband violett.]

- 1897. Ganzsachen. Ebda. (1847). V111,

309 S. m. Abb. 8\%. [Linband violett.]

1898/1899. Marken und Ganzsachen.

Huda. (1892). XXIV, 656-VI11,319S.m. Abl), s". [Finband gelb.]

- 1898/1899. Gimzschen. Ebda. (1898).

VIll, 310 S. m. Abb. $8^{\circ}$. [Hinband gelb].

_ - 1899. Marken und Ganzsachen. Eibda. (1899). XX, 696 + V1Il, 385 S. m. Alb. So.

[Einband purpurrot.]

- 1899. Ganzsachen. Ebda. (1899). VIII, 335 S. m. Abu. 8\%. [Einband purpurrot.] 
Senf, Gebriider: G. S.'s illustrierter Postwertzeichenkatalog 1900. Enthaitend eämtliche Marken und Ganzsachen. Ebda. (1900) XVI, 736 - VIII, 352 S. m. Abb. $8^{\circ}$. [Einband weil, rote Schrift.

- 1900. Marken und Ganzsacben. Kleine Ausgabe. Ebda. (1900). V111, 472 S. m. Abl. $8^{\circ}$. [Einband weiB, rote Schrift.]

- 1901. Marken und Ganzsachen. Fibda. (1901). XVI, $768+$ VIII, 368 S. m. Abb. $8^{\circ}$. [Einband weiB, blaue Schrift.]

- 1901. Marken. Ebda.(1901]. XV1, $768 \mathrm{~S}$. m. Abb. $8^{\circ}$. [Einband weib, blaue Schrift.]

- - 1901. Ganzsachen. Ebda. (1901). V1ll, 368 S. m. Abb. 80. [Eiub.weiB, blaue Schrift.]

- - 1902/1903. Marken und Ganzsacben. Ébda. (1902). XVI, $816+400$ S. m. Alub. $8^{\circ}$. [Einband zinnoberrot.]

- - 1902/1903. Marken. Ebda. (1902). XVI, 816 S. m. Abb. $8^{\circ}$. [Einband zinnoberrot.]

- 1902/1903. Ganzsachen. Ebda. (1902).

400 S. m. Abb. $8^{\circ}$. [Einband zinnoberrot.]

- - 1903/1904. Marken und Ganzsachen. Fibrla. (1903). XXVI11, $888+435$ S. m. Abb.

$8^{\circ}$. Einband blau.

-1903/1904. Marken. Ebda. (1903). XXV111,

888 S. m. Abb. $8^{\circ}$. [Einband blau.]

- 1905. Marken und Ganzsachen. Ébda. (1904). XXVIII, $948+456$ S. m. Abb. 80. [Einband braun.]

- - 1905. Marken. Ebda. (1904). XXVIII, 948 S. m. Abb. 80. [Einband braun; Luxusausg. olivgrün.]

- - 1905. Ganzsachen. Ebda. (1904). $456 \mathrm{~S}$ m. Abb. 8\%. [Einband braun.]

- 1906. Marken und Ganzsachen. Ebda. (1905). XXXII, $1032+478$ S. m. Abb. $8^{\circ}$ [Einband grün.

- - 1906. Marken. Fuda. (1905). XXXI 1032 S. m. Abb. 80. [Einband grün.]

- 1906. Ganzsachen. Fibda. (1905), $478 \mathrm{~S}$ m. Abb. 80. [Einband grün; Luxusausg. dunkelbraun.

- - 1907. Marken und Ganzsachen. Lbda. (1906). XXXI, $1144+496$ S.m. Abb. $8^{\circ}$. [Einband violett.]

1907. Narken. Ebda. (1906). XXXI, 1144 S. m. Abb. 80. [Finband violett.]

- - 1907. Ganzsachen. Ebda. (1906). $496 \mathrm{~s}$ iu. Abb. $8^{\circ}$. [Einband violett.]

- - 1908. Reform-Ausgabe. Harkenteil und Ganzsachenteil. Ebda. (1907). XL, $1216+$ 512 S. m. Abb. $8^{\circ}$. [Einband grau.]

- - 1908. Reform-Ausgabe. Markenteil. Ejbda. (1907.) XI, 1216 S.m. Abb. 80. [Einband grau.]

- - 1908. Reform-Ausgabe. Ganzsachenteil. Ebda. (1907.) 512 S. m. Abb. $8^{\circ}$. [Einb. grau.]

- 1909. Reform-Ausgabe. Markenteil und Ganzsachenteil. Ebda. (1908). XL, $1297+$ 544 S. m. Abb. $8^{0}$. [Linband orange.]
Senf, Gebriider: G. S.'s illustrierter Postwertzeichenkatalog 1909 . Finthaltend sämtliche Reform-Ausgabe. Markenteil. Ebda. (1908.) XL, 1297 S. m. Abb. S*. [Einb. orange.] - - 1909. Reform-Ausgabe. Ganzsachenteil. Ebda. (1908). 544 S.m.Abb. 80. [Einb. orange.]

-_ 1910. Markenteil und Ganzsachenteil. Ebda. (1909). $1168+552+$ Anhang 48 S. m. Abb. $8^{\circ}$. [Einband rot.]

- - 1910. Markenteil. Ebda. (1909). XLVI1I, $1168+$ Anhang 48 S. m. Abb. $8^{\circ}$. [Einb. rot.] - 1910. Ganzsachenteil. Ebda. (1909). 552S. m. Abb. $8^{\circ}$. [Einband rot.]

1911. Markenteil und Ganzsachenteil. Eibda.(1910). XLVI11, 1192 + Anhaug 119 S. +576 S. m. Abb. 80. [Einband b]au.]

- 1911. Markenteil. Ebda. (1910.) XLVIII, 1192 + Anbang 119 S. m. Abb. 80. [Einband blau.]

- 1911. Ganzsachenteil. Ebda. (1910). 576s. m. Abb. $8^{\circ}$. [Einbaud blau.]

- - 1912. Markenteil. Ebda. (1911). XL, 1232 + Anhang 120 S. m.Abb. $8^{\circ}$. [Ëinband braun.] - 1913. Markenteil. Ebda. (1912). XXX11I, $399+$ Anhang 104 S. m. Abb. u. loses InbaltsVerzeichnis XVIS. 8*. [Einband grün.]

- - 1913. Ganzsachenteil. [lst nicht erschienen.]

- 1914. Harkenteil. Ebda. (1913). XLVI11, 1184 - Anhang 80 S. m. Abb. 80. [Einband violett; Luxusausg. oliv-violettmarmoriert.]

- 1914. Ganzsachenteil. Bearb. von C. Lindenberg, mit Unterstützung hervorragender Ganzsachensammler. Fbda. (1913). $655 \mathrm{~S}$. m. Abb. $8^{\circ}$. [Einband violett, Luxusausg. olivviolettmarmoriert.]

- - 1915. Markenteil. Hubda.(1914). XLV1II. $1280+48+79$ S.m. Abb. 80. [Einband grau.]

- - 1915. Ganzsachenteil. [Ist nicht erschienen.]

[Infolge Anschwellens des Markenmaterials wurde zwecks besserer Handlichkeit ab 1914 entgegen allen frìheren Ausgaben ein läneres schmales Format $9^{1} l_{g}: 20^{1 / 2} \mathrm{~cm}$ gewählt.]

- 1llustr. Hauptnachtrag zum Senf-Katalog 1915. Enthaltend alle seit Mitte Juli 1914 bekannt gewordenen Neuerscheinungen von Postmarken. Ferner einige bundert Preisänderungen. Mit rund 200 verkleinerten Abb. u. Aufdrucktypeudarstellungen. Ebda. 1915. $96 \mathrm{~S}, 8^{0}$.

Turde militärischerscits beschlagnabmt u. wuBte daher aus dem Handel zurïckgezogen werden.]

- - Umgearbeitete Neuauflage. Ebda. 1916. 36 S. $8^{\circ}$.

[Beide Ausg. 1915 u. 1916 zwecks Einfügung ein. zelner Bläter in den Hsuptkatalog nur einseitig bedr.

- Preisliste für Verwertung von Briefmarken im Verkaufs- oder Tauschwege nebst den einschlägigen Bedingungen der BriefmarkenHandlung G. S. in Leipzig. 7. Aut. Leipzig: Ebda. 1896. 55 S. $8^{0}$. 
Senf, Gebriider: Illustrierter Spezial-Katalog der deutschen Kolonialmarken und der Ientschen l'ostämter in Auslande unter Berücksichtigung del Farben- u. Aufdruckabarten sowie Fehldrucke u. Plattenfehler. Entwertungs-Stempel auf Ziffer- u. Adler-Zeichnung, Schiffsuild und Germaniatype. Ebda. 1904. 32 S. m. 109 Abb. $8^{\circ}$.

- Zweite Aufl. Fluda. 1907. 47 S. m. 197 Abb. 8".

- Taschenbuch für Briefmarken-Sammler 1887. Fuda. 1887. $88 \mathrm{~S}$. Leinw. geb. $8^{\circ}$.

Enthalt u. a. folgende fachwiesenschaftliche Beiträge : Zur Geschichte der Postwerthzeichen. Philatelie (Briefmarkenkunde). Ueber las Reinigen und Einkleben der Marken. Ueber Únkleben der Sazm. lungen. Das Vordruck - Albun. Fiir Ganzsachen. Bammler. Spezial-Sammlungen, Wasserzeichen. Provisorien und deren Sammelwerth. Gebrauchte und ungehrauchte Marken. Franko-Stempel und dereu Sammelwerth. Markentausch. Verwertung dur Doubletten. Uher den Ankauf von Markeu. Essais und deren Sammelwert.

- - 1887. Zweite Aufl. Eluda. 1887. 88 S. lseinw. geb. $8^{\circ}$.

[Der gleiche Inhait wie die erste Autl.]

- 1888. Hbda. 1888. 88 S. Leinw. geb. 80. [1)er gleiche Inhalt wie die $1887 \mathrm{cr}$ Ausgaben.]

- 1889. Kibda. 1889. 88 S. Leinw. geb. $8^{\circ}$.

Euthält u, a. folgende fachwissenschaftliche Beiträge: Die Zukunft der Friefmarkenkunde. Von Ludwig Fischer. - Einiges über das Brief-und Verkehrg. wesen im Altertum. Von Boethius. - Ein Beitrag zur Geschichto des alteren Postwesens. Mit einer Tafel. Von L. Clericus, - Der Wechsel in den Forbildern der Postwertzeichen. Mit Abbid. und einer Geschichtstafe]. Von O. Kausch. - Die Pastkarie, ihrc Lrfindung uud ilhe Verbreitung. Mit Abbildung. Von K. von Güudel. - Die bildl. Darstellungen anf den Dentschen Privatmarken. Nit vieleu Abbildungen. Von W. van Schimuelfennig. - Der 1]te, Erzibluug von Dr. Krauge. - Rätsel.

- Taschenbuch für BriefmarkenSammler 1894. Ebda. 1894. 104 S. m. Abb. Leinw. geb. $8^{0}$.

- 1895. Fluda. 1895. I04 S.m. Aub. Leinr. geb. $8^{\circ}$.

Nenf, Louis: Handbuch für Postkarten-Sammler bearb. von L. S. Verzeichnis aller bis zur Neuzeit erschienenen Postkarten u. Kartenbriefe. Mit vielen erklärenden Anmerkungen u. über 600 Abb. . . Leipzig: W. A. I.ouis Senf \& Co. I890[-91]. 4SS \& . $8^{\circ}$.

(Bibliotbek für Postwerthzeichels-Sammler Bd. Z.) [Ureprünglich in $5 \mathrm{Lfgu}$. ergchienen.]

Katalog aller seit 1653 bis Fnde Dezember 1875 emittierten Briefmarken [u. s. w.] bearbeitet von Dr. Alfred Moschkau. Leipzig: Louis Seuf $1876.250 \mathrm{~S} .8^{\circ}$.

- Naclitr. 1876. 20 S. $8^{0}$.

- s. a. Bibliothek, Philatelistische u. Moschkau, Alfred: Deutscher Philateli-ten- $\mathbf{K a l e n d e r}$ für 1877.

Teuf, Richard: Adressbuch aller briefmarkenHändler der Welt. Leipzig 1874.

- Die Druckverfalıen bei Herstellung der Postmarken. Vortrag. s. Deutscher Philatelistentag; in: Stenogr. Bericht ï. d. XVI. Deutschen Philatelisten-Tag ... in Leipzig (u. Halle a. S.) 1904 .
Senf, Richaril: s. a. Moschkau, Haudbuch für Postmarken-(bezw. I'ustwertzeichen.)Satnm]er. 6. แ. 7. AuH. 1890-

Serbe, C. Ilermann: 2150 Adressen der Brielmarken-Händler und -śammler allel Länder. 2. Aufl. Leipziğ: O. Herm. Serbe 1888. 2 ".

sickmaun A lirauschweig: Preisliste aller Postmarken usw. Lübeck 1872. 8\%.

- Naclitr. Ebila. 1873. 8".

Nieblist, Otto: Die Post im Auslaude. Darstellung der Posteinricbtungen des Auslaudes, nach amtI. Quellen tearb. 2. Aufl. Berlin: Julius springer' 1892. 450 S. m. I 'l'abelle. Leinw. geb. $8^{\circ}$.

— - 4.Aut. Ebda. 1909. ca. 500 ๖. m. 1 'Tabelle. Leinw. geb. 80.

siewert, J.: Wie Chalmers-Hill-liage. s. Evans, E. B.

Shandinavien. Audresen, Ferd. jr:: Preisliste über Skandinavische Briefmarken. Christiania $189 \%$.

- hommel, Utto: Die Privat, Eisenuabn- u. Dampfschiftsmarken von Skandinavien und Finnland. Gössnitz 1909.

- 'Tybring, Julius: 1887. Preisliste über shandinavische Briefmarkeu. Kopenhagen 1887.

Som luerfeldt. Willy: Die als Dienst formulare verwendeten überdruckten Kreuzel-Postkarten von Württemberg. (Sonderabdr. aus der Festschrift zur Feier des 25 jähr. Bestebens des Berliner Philatelisten-Klubs.) Berlin: Berline $1^{\circ}$ Philatelisten-Klub 1913. 16 \$. m. Alub. 8".

- - s. a. Berlin (Berliner Philatelisten-Klub); in: Festschrift z. Feier des 25 jähr. Bestehens des B. Plı.-Kl.

- Die Feldpost-Gianzsachen der OPD. (Oberpostdirektion) Strabburg. (Sonderabdr. aus "Vertlaul. Mitteilungen ${ }^{*}$ des Berliner Philatelisten-Klubs 1915. Berlin: Berliner Philatrlisten-Ǩlub 1915, 2 Bl, 4\%.

Soesman, F. J. II.: Spezial-'Tausch- u. Verkauts-Offerte. F. J. H. S. Samarang, Java 1887. 11 s. 80 .

souvenir. Yhotographisches, für Dentschlauis Philatelisten. s. Moscbkau, Alned.

spunien. Spaniens Bầ (Fälschung); in: Mosch. kau, A., Zur Geschicbte der Philatelie.

- Friederich, R.: Die Postwerthzeichen Spanims u. seiner Colonien. T'. I. Mähr. Ostrau 1890.

- - 2. Aufl. 2 'l'eile in I Bd. Berlin 1894.

- Glasewald, A. E.: Don Carlos-Marken. Torlrag. Gössnitz S.-A. 1915.

- Kohl, Paul: 1llustr. Katalog der Freimarken ion Europa. Abt. 9. Spanien". Chemnitz $1894 / 95$.

- Lietzow, Paul: Spanien. Post-u. Verkehrs. wesen auf der pyrenäischen Halbinsel. Berlin 1891. 
Speiser, I.: Von der Eeldpost zux Postreform. 1. Postfleiheit. "2. Die Weitformat-Postkarte. 'Wwei Anregungen. Leipzig: Carl Erust Poeschel in Komm. 1915. $31 \mathrm{~S} .8^{\circ}$.

Sipekulation. Brummer, Leon: Die Philatelie im Dienste der Spekulation. Vortrag. s. Deutscher Philatelistentag; in: Stenograph. Bericht ïber die Verhaudlungen des VII. D. Ph. zu Mannheim 1895.

Siezial-Kataloge n. l'reislisten für Altueutschland- u. Huropa-Sammler. Eckstein, Dr.: Altdeutschland-Katalog. Aachen: Selbstverl. 1913. $48 \mathrm{~S} .8^{\circ}$.

Freudenstein, S.: Katalog altdeutscher Postwertzeichen. Leipzig 1891.

[s. a. ${ }_{n}$ Dauth, H. J. ${ }^{4}$ u. ${ }_{n}$ Gust, R. ${ }^{\mu}$ uuter gleiclinamigem Titel gedr.]

Freyse, Gustav: l'reisbuch Europa*. Han. nover 1905

- Europa-Katalog. llannover 1907-1910.

- Vriedemann, A.: Briefmarken-Preisbuch Europa. Leipzig 1908-1914.

- Glasewald, A. E.: Preisbuch „Europa ete. Gössniť, S.A. 1890-1904.

- Kohl, P.: P. K.'s illustr. Katalog der Frejvarken von Deutschland. Chemnitz 1897.

- P.K.'s illustr. Katalog der Freimarken von Europa. In 9 Abt. Chemnitz 1894-95.

- Freimarken-Katalog 1900. Bd.1: Europa, Europäische Kolonien*. Chemnitz $1900 / 01$.

- Köhler, H.: Preisliste u. Gelegenheits-Offerten vorräiger Briefmarken. Berlin 1915.

Kosack, Ph.: Berliner Briefmarken-Katalog. Ausg. 1909. T. 1. Ber]in [1908].

- - [Nr. 24.] Ausg. 1917. Fbula, 1917.

- Krötzsch, H.: Illustr. ausführl. Katalog über Deutsche Postfreimarken. Leipzig 1896.

- Lublin, G.: Katalog für Briefmarken-Sammler Nr. 18. Separat-Abdruck der Deutschen Slaten. Berlin [1892].

Maier, M. K.: Preisliste 1917. Berlin 1917.

Jarken- u. Ganzsachenhaus G. m. b. H.: Die neue Preis-Grundlage 1917 bis 1918. Berlin 1917.

Michel, Hugo: Huropa-Katalog [nebst Nachträgen]. Apolda 1910-1915 und Weimar $1916-1917$.

- Michel's Mignon. Apolia 1914-1915 u. Weimar 1916.

- Rommel, 0.: Special-Catalog der Postwertzeichen der Deutschen Postbezirke. Dresden 1896.

- Ruben, Edv. MI: Briefmarkenpreisliste Nr. 15 [Ausg.] 1917. [Auch u. d. T.: ${ }_{\text {N }}$ Neue EuropaPreisliste".] Berlin 1917.

- Willadt, Car], \& C'o.: Europa-Special-Preisbuch. Pforzheim 1907-1914.

Zschiesche, Alwin: Katalog sämtlicher bis 1891 ausgegebenen Couverts, Postkarten 1. Kreuzbandstreifen der dentschen Starten. Naumburg a. S. 1891.
Spezial-Kataloge u. Preislisten für Altdeutsch. lank- u. Furopasammler. Zschiesche, Alwin: Katalog über alle bis 1896 ausgegebenen Briefmarken von Europa. Naumburg [1896].

- Zumstein, Frnst: Kuropa-Katalog. Bern 1911 u. 1912.

Spezialsammlungen. s. Ist es vom philatelistischen Standpunkte...

- s. a. Metzkes, Einst.

Statspost u. Privatpost. s. Göpfert, li.

Nialler, Erieh: Die Postordnung fül dias Deutsche Reich vom 20. Mälz 1900. l3erlin: Carl Heymanns Verlag 1914.

Siadthagen: Mitteilungen ijber philatelistische Prozesse. s. Berlin (Berliner PhilatelistenKlub); in: Festschrift z. Feier des zelınjähr. Bestehens des B. Ph.-K1. Berlin 1898.

Stadtpost Warschau. s. Zustellungsmarken.

Stäger, A.: Die eillgenössische Post. (Sonderabdr. aus: Jahrbuch der schweizerischen Fidgenossenschaft.) Bern: K. J. Wyss 1911. 62 s. m. I Bild. $8^{\circ}$.

Stäger, Joh. Ant.: Dis schweizerische Postwesen zur Zeit der Helvetik. Nach offziellen Quellen bearb. 2. Ausg. Bern 1879. 8\%.

Nitielile, Emil: Katalog und Preisliste der Postwertzeichen Ceylons. Mit Randbomerkungen u. Erläuterungen. Mannheim: Selbstverl. 1896. $23 \mathrm{~S} .8^{\circ}\left(16^{\circ}\right)$.

Starke, Konrad: Kalender für Briefmarkensammler für das Jahr 1895. Dortmund: Briefmurkensammler-Verein 1895. $192 \mathrm{~S} .8^{\circ}$.

Statistik. Keller, A.: Statistische Tafel mit verschiedenen Vergleichstabellen. Zsgest. für Freunde der Briefmarkenkunde. Miinchen 1884.

- Krötzsch, H.: Statistik über Stückzahl und Wert meiner Postwertzeichen-Sammluug. Leipzig [1916].

iber Stückzahl u. Wert meiner Postwertzeichen-Sammlung. Wien, XVIII., KarlLndwigstrasse Nr. 7: Karl Hudetz o.J.

[2. u. 3. Aufl. 8, u. „K Kötzscb, Hugo ${ }^{66}$.]

Steinberg, L.: Preisconrant über Briefmarken von Rumänien. Bulkarest: Selbstverl. [1895]. $12 \mathrm{~S} .8^{\circ}\left(16^{\circ}\right)$

stempelmarken. Perlep, A.: Katalog der Stempelmarken aller Länder. Leipzig 1880.

- Das Sammeln ron Stempelmarken; in: Moschkau, A.: Zur Geschichte der Philatelie. Wiilbern, Arthur: Illustr. Preis-Katalog der Stempel-u. Telegraphenmarken des Deutschen heiches u. seiner Einzelstaaten. Hamlurg 1912.

Stenger, Eriell: Bayern. (Sonderdr. aus „Der Fanzsachen-Sammler. ") Berlin: Philipp Kosack 1912. $4 \mathrm{~S} .4^{\circ}$.

- Bayern $9 \mathrm{Kr}$. grüu. 'l'y penunterschiede. (Sonderdr. aus "Germania-Berichte *) [Leipzig] Berlin: Selbstverl. des Verfassers. 1914. 4 S. $8^{\circ}$. 
Stenger, Erich: Bayern - Feldpost 1914. (Souderdr. d. A. Berliner Hriefmarken-Zeitung Berlin: Philipp liosack \& Co. 1914. 3 S. 4*.

- Bayern - Portomarke 3 kr. schwarz mit Seiclenfaden. (Sonderdi. a. d. ,Berliner I’́riefmarken-Zeitung".) lierlin: Plílim liosick 1910.8 S. $4^{\circ}$.

Dio Bewertung der alldeutschen Marken auf Brief im Neuen Normal-Katalog. (Sonderdr. aus der Berliner Briefm.-Zeitung".) Herlin: l'hilipp Kosack \& Co. 1913. 3 S. 4".

Die Bogeneinteilung u. die Auflagezalılen der Brief- u. Portomarken Bayerns. (Sonclerdr. a. d. Berliner Briefmarken-Zeitung ${ }^{\circ}$.) Berlin: Philipp Kosack 1911. TS. 4\%.

- Essais und Proben der Brief-, Porto-, 'Telegrapben- and Stempelmarken von Bayelu. (Sonderdruck aus der Festschrift des Berliner Philatelisten-Klubs 1913*.) Berlin: lierliner Philatelisten-Klub 1913. 18 S.n. Alıb. $8^{0}$.

- Essais u. Proben der Brief-, Porto-, 'Telegraphen-, Stempelmarken n. Ganzsachen von Bayern. (Sonderdr. a.d. Berliner BriefmarkenZeitung.) Berlin: Philipp Krosack \& Co. 1912. 35 S. $8^{\circ}$.

- Die objektive Feststellung der Wasserzeichen in scbwierigen Fillen. (Sonderur. a. d. Berliner Briefmarken-Zeitung".) Firda. 1914. $2 \mathrm{~S}, 4^{\circ}$.

- Teschichte u. Kataleg der Postanweisungen von Bayern. (Senderdr. aus, Germania-Berichte".) Leipzig: Germania-Ring. 1914. 52 S. $8^{\circ}$.

- Die rote frammierung bei Bayern-Marken. (Sonderdr. a. d. "Berliner Briefmarken-Z/tg. ${ }^{*}$ ) Berlin: Philipp Kosack \& Co. 1914. 2 S. 40.

- Die Güterexpeditionsstempel von Bayern als Briefstempel. (Sonderdr. a. d. Berliner Briefmarken-Zeitung ${ }^{\circ}$ ) Ebda. 1915.2 S. $4^{\circ}$.

- Uberklebte Marken von Bayern. (Sonderdr. a. d. Berliner Briefmarken-Zeitung".) Ebda. 1913. 2 S. 40.

- Pholographische Methoden im Dienste der Briefmarkenkunde. (Sonderd1. aus der ${ }_{n} \mathrm{Ber}^{\circ}$ liner Brietmarken-Zeitung") Berlin: Plilipp liosack 1909. $14 \mathrm{~S} .4^{\circ}$.

- Die Mühlradstempel von Bayern. (Sonderdr. aus ,Germania-Berichte".) [Leipzig] ๖. Berlin: Selbstverl. d. Verf. 1915. 8 S. 8".

-- Die Postkarten von Bayern. Hin Nachtrag zu Franz Schneider's Katalog nebst Preisverzeichnis. (Sonderds. a d. Borliner Briefmarken-Zeitung".) Berlin: Philipp Kosack \& Co. 1915. 2 s. $4^{\circ}$.

[8. a. Schneider, Franz: Katalog der Bayerischen Postkarten.]

- Speknation in Bayern-Marken. Sonderdr. aus der ,Berliner Briefm.-Zeitung*.) Fbda. 1914. 2 S. $4^{\circ}$.

itenger, krich n. Ludwig Sauter: Die Postablagen, Landposthoten und Posthilfstellen. Stempel von Bayern. Ebda. 1915. $96 \mathrm{~S} .8^{\circ}$.
Stephan, Der kleine. Fin Hilfsbuch für's Publikum. lid. 1. Ausg. fiir 1901. Jlhustr. beutsches Post- und Telegraphen-Handbuch für den gesamten In- 11. Auslands-Verkehr, nebst einem Verzeichnis der Nachbarpost. orte, Bestimmungen über den lernsprecl. verkehr u. posialischen Sirafenverzeichnis von Berlin. Neueste Aut. (abgeschlessen am 15. April 1901). Nach den amtl. Feröffentlichungen der lieichspostverwattung hesorgt von Lipski, Ober Pestdirektionssekretair. Dresden: Gerhard Kühtmann 1901. IX, 164 S. 8"

[Dio nenesten Ausg. 8. u, dem veribul*rton Titel ${ }_{n}$ Kiilıtmann's Posthandbuch".]

Stephan, Ernst HeinrichiWilhelm von: Biographie; in: Jeran, O.: Wegweiser fü $\mathrm{V}^{\mathrm{P}} \mathrm{r}$ kehrswertzeichen Sammler.

- auch ju: Moschkau, Alfr(4l: \%ul' lip. scluichte der. Philatelie.

s. femer auch selbständige Schriften von: Billig, B.; Krickeberg, H. u. Hoschkau, Alfred.

- Frgänzung der Geschichte der Correspondenz. liarten. Berlin: [Reichsprostmuseum] 1865. Als IIs. gedr. $2 \mathrm{~S} .4^{\circ}$.

- Treschichte der Preussischen Pest von ibrem Irsprunge bis zur Gegenwart. Naclı amtlichen Quellen. Berlin 1859. 8\%.

- Weltpost u. Luftschiffabrt. Fin Vortrag im wissenschaftlichen Verein zu Berlin gehalten. Berlin: Julias Springer 1874. 75 S. $8^{\circ}$.

Sternheim, Carl: Katalog der deutschen Privatpost-Marken. Bearb, u, wit Durchschnittspreisen versehen von C. S. SchönebergLerlin: Selbstrer. 1902. V11, $124 \mathrm{~S} .8^{\circ}\left(16^{\circ}\right)$.

- - Nachtr. Ebda. 1904. 1V, 17 S. $8^{\circ}\left(16^{\circ}\right)$.

- Zweite Aut. Berlin: Philipp liosack 1909. $149 \mathrm{~S} .8^{\circ}$.

Stiegler, Josef: Adress. und Handbuch fül Philatelisten. Wien $\mathrm{X} \mathbf{X} / 2$, Dresdenerstralie 80 : J. Stiegler 1912....S. 80.

Inhalt: Sammler, Händler-, Zeituugs, V'ereins-, Tezugequellen. Verzeichnis. - Briefpost. Geldbrief-, Postanweisungs Tarife, - Notizkalender, Minztabelle, statistık uiber den Wert der Sammlungen, Briefsteller iu 6 sprachen etc. etc.

Stock, Erust: Raritätentafel Nr.1-8. Berlin: Frust Stock 1901. $8^{\circ}$.

[Abbildungen gesuchter Seltonhesten.]

Stockholm (Sveriges Filatelist-Förening). s. Verzeichnis über die Postwertzeichen von Schweden.

Storch, Adolf Fr.: Der Briefmarkenfreund. Ill. Beschreibung aller Briefmarken der Frde. Wien: Selbstrerl. d. Verf. [auch, $V e r l a g$ der Post * in Komm.] 1866. $43 \mathrm{~S} . \mathrm{S}^{\circ}$.

- Post- und statistische Tabelle. Hrsg. u. verlegt von Adelf Fr. Sterch, K. K. Posteffizial u. Redakteur. Wien: Selbstrerl. 1866. 1 Bl. m. $98 \mathrm{Abb}$. von Postwertzeichen. $2^{\circ}$.

Strochlin, l'aul. s. Erdmann, A.: Die Postmarken ron Griechenland. 9. Autl. [Übelsetzer clieser Ausg. in's "Französische*.| 
Nucki, A.: Grundriss der lostgeschichte mit besonderer Berücksichtigung der schweizerischen Verkehrsverhältnisse. Bern: A. Francke 1909. XII, 168 S. $8^{\circ}$.

Stnmu-Halberg, Frlı. Carl Ferdinaud vou: Dns Postwesen des Deutschen Reiches 1880 bis 1891. (Aus des Freilherrn Carl Ferdinand von Stumm-Halberg Reden Bd.6.) Berlin: Otto Elsner 1910. X], 384 S. Sn.

stiind, Johann Wilhelm: Die Abstempelungen auf den Postwertzeichen von Bayern. Verzeichnis der Mühlradstempel nach Orten und Nummern. Leipzig: Hugo Krötzsch 1917. 47 S. m. 2 Ablu, im T'est. $8^{\circ}$.

Stultgart (Wiuttembergischer PhilatelistenVerein). Bericht über die Feier des zelınten Stiftungsfestes und die damit verbundene Postwertzeichen - Ausstellung. Stuttgart: Selbstverl. 1892. 8S. 4".

sualeli. Haas, Theodor: Die Postwertzeichen des Suaheli-Sultanates. Berlin 1898.

siidamerika. Caspart, Rudolf: Ein philatelistischer Streifzug durch die südamerikanischen Unabhängigkeitskriege. Vortrag. Wien 1911 (Hamburg 1912).

Sudan. 8. Pleimes, Alphonse: Kngros-Preiskurant von allen Marken von Sudan nud Aegypten.

Suppantschitsch, Victor: Biographie in: Jeran, O.: Wegweiser für VerkehrswertzeichenSammler.

- Beck, Carl; Senatspräsident Victor Suppantschitsch. Altmeister der österreichischen Philatelie. Biographische Skizze. (Sonderdr. a. "Der Philatelist". Dresden, Juli 1917.) Berlin: Selbstverl. 1917. 4 S. nebst Portr. 8\%.

- Bibliographie zugleich Nachschlagehuch der gesammten deutschen philatelistischen Literatur seit ihrem Entstehen bis Ende 1891 nehst einem Abriss der Geschichte der Philatelie mit hesonderer Berücksichtigung Deutschlands und einer kurzen Geschichte der deutschen philatelistischen Literatur. München: $\Lambda$. Larisch 1892 [-94]. 749 S. 8".

[Ursprianglich iu 16 Lfgn. erschienen]

- Die Entstehung und Entwickelung der philatelistischen Literatur in der zweiten Hälfte des XIX. Jahrhunderts. Wien: [Selbstverl.] 1901. $63 \mathrm{~S} .8^{\circ}$.

- Grundzüge der Briefmarkenkunde und des Briefmarkensammelns. Mit dem Portr. Rowland Hills u. 7 in den Text gedr. Abb. Leipzig: J. J. Weber 1895. V1II, $221 \mathrm{~S} .8^{\circ}$.

[Wehers Ill, Katechismen Nr. 152.]

- Katalog der philatelistischen Bibliothek des Oherlandesgerichtsrates Victor Suppantschitsch in Graz bis Ende 1893. (Sonderdr. aus ,Austria-Philatelist" Alärz 1895 bis .Juli 1897.) [Graz: Selbstver]. 1897.] 34 S. $8^{\circ}$.

- Leitfaden der Philatelie (Briefmarkenkunde). Ein unentbehrlicher Ratgeber für angeliende Philatelisten, sowie für fortgeschrittene Sammler, Leipzig: Fd. Wartig 〈Ernst Hoppe〉 1880. IV, $136 \mathrm{~S} .8^{0}$.
Suppantschilsch, Victor: Die philatelistische Literatur Österreich-Ungarns im XlX. Jahrhundert. (Sonderdr. aus "Der Philatelist", Januar-Mai 1909.) Dresden: Intern. Philatelisten-Verein 1909. $8 \mathrm{~S} .8^{\circ}$.

Die Seltenheiten der philatelistischen Zeitungsliteratur bis Ende 1900. (Sonderdr. aus Der Philatelist" Juni 1903 his Februar 1904.) Wien [u. Dresden: Intern. Philatelisten-Verpin] 1904. $12 \mathrm{~S} .8^{\circ}$.

Sivenson, W.: Typen und Abarten der Wendenschen Originalmarken, offiziellen Neudrucke, Nachdrucke u. Fälschungen. (Sonderdr. aus "Berliner Briefmarken-Zeitung" Februar bis Juni 1907.) Rerlin: Philipp Kosack 1907. 11 S. u. 4 Taf. m. Abb. $4^{\circ}$.

[5. a. Hirschheydt, A. จ.: Fin neuer Schluss zum Artikel "Wenden".]

Nreriges Filatelist-Förening, Sloekbolm. s. Verzeichnis iber die Postwertzeichen vos Schweden.

Nzekula, Béla: Almanach 1903-1904. Thenf: Selbstver. 1903. $72 \mathrm{~S} .8^{\circ}\left(16^{\circ}\right)$.

- Almanach 1905-1906. Budapest: Selbst.verl. 1905. $160 \mathrm{~S} .8^{\circ}$.

- Almanach 1907. Fbda. 1906. 144 S. 8*.

- Almanach Internationale Philatelisten “ (Philatelistes Internationaux) 1908. Budapest: Béla Szekula 1908. 128 S. $8^{\circ}$.

Inlalt: Philatelistischer Briefsteller in 11 Spra. chen. - Wörterbuch! - Adressen von SammlerFreunder (Blanko). - Statistik für Postwertzeichensammlungen. - Annoneen der Mitglieder der Internationalen Philatelisten" mit photograph. Aufnahmen. - Reklameteil. - Rlankobogen fïr den Tausch- u. Kaufverkehr. - Umsehlag mit Übersichtskalender.

Tabelle, Chronologische, der deutschen philatelistischen Zeitschriften. s. Fränkel, Heinrich.

Tafel, Statistische, mit verschiedenen Vergleichstabellen zsgest. f. Freunde der Briefmarkenkunde. s. Keller, A.

Tamsen, Emil. Nitarbeiter am, GroBen Handbuch der Philatelie". s. u. Natal", "Neue Republik".

Tarif und Beförderungs-Bestimnungen dè" Neuen Berliner Omnihus- und PacketfahrtActien-Giesellschaft. Gegründet 1884. Berlin S.: Selbstverl. 1892/95. 18S. $8^{\circ}$.

Taschenatlas f. Briefmarkensammler. s. Friedl, Rudolf.

'I'aschenbuch fü. Briefmarkensammler. s. Srnf, Gebr.

- für Philatelisten (1911). Wien: Verlag der Fachschrift für Philatelie $u$. Sammelwesen: "Der Universal-Anzeiger" 1911. 80.

Inhalt: Warnungstafel mit 300 Namen, Minz. tabellen, Belletristik, Prämienkupons, Legitimations. soite etc.

Tauschadresseu in: lsakovics, A. v.: Internationales 'Tausch-, Adress- $u$. Hïlfsluuch fiir' den Tauschverkehr. T. 1.

[s. a. Adressbücher.]

Tanschverbindungen. Glasewald, A. E.: Wie verbessern wir unsere Tauschverbindungen? Vortrag, geh. auf dem V1l. Dentschen Philatelisten-Tag zu Mannheim 1895. 
'lauschrercinigumgeu. (thasewald, A. E.: l)i Kuknntt unserer 'lauschvereinigungen. Vortrag, geh, auf dem Xll. Deutschen l'hilatelisten-I'ag. Frankfurt a. M. 1900.

'I'assehrerkehr. I.eopold, Ernst: H. I.'s lland und Hilfsbüchlein fül' den 'l'auschverkehr. livemen 1893.

'Tauseliverkehrsbuclı für Postwertzeichensammler. s. Sehmidt, Gustav.

'Tavouklji, 1’uul: Im Oktober 1887. PreisListe Nr. 1 n̈ber orientalische Briefmarken. Constantinopel 1837. 12 S. $8^{\circ}$.

Taxis-Taxen s. Thurn u. Taxis unter Schloss, H. J.

Teichmann, Ir:: Schutz den Ganzsachen(Sonderdr. aus den Mittheilungen d. Oester. reichischen Philatelisten-Clubs ${ }^{*}$ v. 14. Dezbr. 1889.) Dresden: [Selbstverl. 1890]. 4 S. 8\%!

T'eiehmann, M.: Handbuch für Briefmarkenn. Ansichtskarton-Sammler. Leipzig: [Selbstverl.] 1898. $40 \mathrm{~S} .8^{0}\left(16^{\circ}\right)$.

Telephonmarken von Belgien. s. Misson: Geschichte der telephonischen Marken von Belgien.

Telegraplenuarken. Kalckhoff, Franz: Die 'T'elegraphenmarken u. Fernsprechscheine der Deutschen Staten. Berlin 1910.

- Die Preussischen Telegraphen-Marken: Berlin 1898.

- Verzijl, Jean: Illustrierter Telegraphenmarken-Katalog. Louvain [u, Berlin] 1912.

- Wiilbern, Arthur: Illustr. Preis-Katalog cler Stempel-u.'Telpgraphenmarken des Deutschen Reichesu. seiner Einzelstaaten. Hawburg 1912.

Teltz, 01to: Großes Handbuch der Philatelie. Ursprüngliche lieferungsansgabe. Leipzig, Frnst Heitmann 1887 [-97]. $8^{\circ}$.

Lfg. $1-3$ bearl, von Otto Teltz.
$4-20, \quad$ Carl Lindenberg.
" $21-27 \%$ Freiherr von Vitting holf-Schell, J. H. Anheisser u. Dr. Franz Kalckhoff.

28-37 bearb, von Ernst Heilmanu.

[Die näheren bibliograph. Einzelheiten 8. u dem fresant-Titel: "Handbuch, GroBes, der Philatelie". s. a. u. Kalckluaf, Frauz: Grosses Handbuch der Philatelieu. 2. Aufl.]

- Wie wird wan Philatelist? Eine Anleitnng zu Nutz und Frommen jüngerer Sammler. s. Philatelia (Verein) in: Vereinsmittheilung der Philatelia*. Sondershansen 1887.

'Thalmau, Friedrich Wilhelm: Bücher-Verzeichnis der Zentral-Bücherei des GermaniaRinges, Hannover: Germania-Ring 1907. 8 S. $4^{0}$.

- Nachtr. Hbda. 1907. 5 S. 4\%.

- Fålschung Preussen 2 Silbergroschen ultramarin (Ausg. 1861/65) postalisch gebraucht auf Brief. (Sonderdr, aus den Germania-Berichten 1916.) Leipzig: Germania-Ring 1916. 14 S. m. Abb. $4^{0}$.
'Thalmaun, Friedrich Wilbeln: Kriegs. Ortspost-tempel. (Sunderabdr. aus den GermaniaBerichten 19l6) Euda. 19l6. 2 S. m. A bb. 4".

Die Potsdam. Poststempel-Sammlung des Vereins der Briefmarkensammler zu Potslam auf der Internationalen Postwertzeichen. Ausstellung in Cassel. (Sonderabdr. aus den Gelmania Berichten 1914. H.7/8.) Wbda.1914. 7 S. m. Abb. 4".

- Ansführliches Verzeichuis der ZentralBücherei des Germania-Ringes. Mit zahlreichen biblingraphischen Hinweisen. Essen a. d. Ruhr: Germania Ring 1912. $105 \mathrm{~S} .4^{0}$.

- Verzeichnis der in philatelislischen Zeitschriften $\langle\mathrm{J}$ ahrgang 1910$\rangle$ enthaltenen Fachartikel und der in den Jahren 1908-1910 erschienenen Handbüclıer. Ébda 1911. 15 S. 4 .

Theinert, H.: Katalog des Reichs-Postmusenms. Im Auftrage des Reichs-Postants bearbeitet. Berlin: Julius Springer 1889. $372 \mathrm{~S} .8^{\circ}$.

$$
\text { [в. в. и. "Berlia (Reiohspostmuseum)".] }
$$

Thicle d Co.: Vollständiger Catalog über alle his jetzt erschienenen Briefmarken, welche zu den beigesetzten Preisen bei $O$. Thiele \& Co. in Mannheim zu haben sind. Mannheim: Selbstverl. 1865. 25 S. $4^{\circ}$.

'Thier, Iax: Kurzgefasste Besprechung der Briefmarken-Sammlungen von Martin Schroeder, Leipzig. Leipzig: Martin Schroeder 1903. 72 S. $8^{\circ}$.

- Gegen das Ausschneiden von Ganzsachen. s. Deutscher Philatelistentag, Antrag 4; in: Bericht ü. d.... II. Deutschen PhilatelistenTag zu Frankfurt a/M. 1890.

'Thurn d Taxis. Der Begründer des deutschen Postwesens Lamoral $v$. Taxis; in: lsakovics, A. v.: Internationales Tausch-, Adress- und Hülfsbuch für den Tauschverkehr 'T. 2.

- Fisbaeh, Friedrich Carl: Taxis, Fesischrift. Regensburg 1915.

- Glasewald, A. E.: Die Abstempelungen der Marken des Thurn u. Taxis'schen Posigebietes. Gössnitz 1893/94.

- Grosse, Oskar: Die Beseitigung des Thurn u. Taxis'schen Postwesens in Deutschland durch Heinrich Stephan. Minden 1898.

- König, Fmil: Scbwarze Kabinette. Mit Anlagen: Geschichte der 'Thurn u. Taxis'sthen Postanstalt und des österreichischen Postwesens. Brannschweig 1875.

- Lindenberg, Carl: Die Briefumschläge von Thurn u. Taxis. Berlin 1893.

- Mehler, J. B.: Das fürstliche Hans Thurn u. Taxis in Regensburg. Regensburg 1899.

- Ohmanu, Fritz: Die Anfänge des Postwesens u. die Taxis. Teipzig 1909.

- Rühsam, Josef: Postgeschichtliche Dokumente aus dem fürstlich Thurn u. Taxisschen Archiv. 1504 bis $1866\langle 1909\rangle$. Berlin 1910. 
Thurn \& 'Iaxis. Rübsam, Josef \& Rudolf Freytag: Postgeschichtliche Dokumente des Fürstlich Thurn u. 'l'itxisschen Zentralaichivs zu Regensburg $\langle 1504-1909\rangle$ auf der Intern. Ausstellung f. Buchgewerbe und Graphik zu Leipzig 1914. Regensburg 1914. Als Mls. gedr. - Schloss, J.H.: Taxis-Taxen. Verzeichnis der Nummern-u. Orts-Abstempelungen von Thurn u. Taxis, Frankfurt a/Main: Selbstverl. 1907. $10 \mathrm{BI} .8^{\circ}$.

[Ursprianglich als Preisrerzeichnis für Tuxis - Abstempeluugen gedacht. Erschien jedoch ohne* Preise einseitig bedr, u. rückseitig gummiert $z$ wecks Aus schneidens der eiuzelnen Yostorte u. Einklebevs in eiue Sondersammlung.]

- Ton, Max: Die Thurn u. Taxis'schen Postfreimarken. Regensburg 1911.

Tiffany, John Kerr: Fehl-Liste der Abtheilung ron "Deutsche Werke". St. Louis, Mo. 1894. 26 S. $8^{\circ}$.

'Tobler, Alolf: Der Kampf gegen das Fålscherwesen. Vortrag, gehalten an 31. Juli 1914, anläBlich des Schweizer. Philatelisten-Tages in Bern. (Separat-A bzug aus der Schweizer Briefmarkpnzeitung " 1914.) Bern: Zumstein \& Co. 1914. $8 \mathrm{~S} 88^{\circ}$.

'Tou, Max: Der Briefmarken-Sammler. Leipzig: Verlag für Kunst u. Wissenschaft Albert Otto Paul [1917], 64 S. 80.

(Miniatur Bibliothek Bd, 278-279.)

- Handbuch der deutschen philatelistischen Literatur. Im Auftrage des Internationalen Philatelisten-Vereins Dresden unter Mitwirkung von Oberlandesgerichtsrat Dr. W. Berchelmann, Darmstadt - Redaktenr A. F. Glasewald, Gössnitz - Geh. Regielungsrat Dr. F. Kalckhoff, Berlin - Geh. Justizrat Emil Pauli, Berlin - Dr, med. Paul Pirl, Charlottenburg - Hrnst Plotz, Dresden Dr. jur. O. Romunel, Berlin - K. K. Senats. pricident i. R. Viktor Suppantschitsch, Graz - Franz IVillner, Blasewitz-Dresden, hrsg. u. bearb. von M. T. Iresden-Blasewitz: Franz Wallner 1916. 〈Als Manu-kript gedr.> Vollendet Frbruar 1918.] IV, 144 Spalten $8^{\circ}$.

- Aitcleutsche Postwertzeichenkunde. Ein Leitfaden fïr Postwertzeichensammler. Leipzig: Verlag für Kunst $\mathrm{u}$. Wissenschaft Albert Otto Paul [1913]. 174 S. $8^{\circ}$.

(Miniatur-Bibliothek Bd. 1059-1062.)

- Die Thurn u. 'Taxis'schen Postfreiwarken, ihre historische Bedeutung und ihr Sammelwert. Erfahrungen u. Vors hläge ïber das Ordnen u. Katalogisieren der Thurn u. Taxisschen Postfreimarken nebst einem Versuch über die richtige Bewertung der vorkommenden Abstempelungen. Regensburg: Georg Brand 19!1. $44 \mathrm{~S}$. 80.

[Die Reatan H 350 Expl. ging im Juli 1917 käuflich an die Briefmarken- und Verlagsbuchhandlung Frau Eugenie Müller, Müuchen, Theresienstr. 57 üher.]

Tonga. Kobl, Paul: lllustr. Froimark-n-Katalog "Englische Kolonien" Abt. 3 ... Tonga.

Töpfer, J. A.: Garantiestempel. Vortrag. s. Dentscher Philatelistentag; in: Bericht ü. d. IV. D. Ph. in Prag 1892.

Toscana-Marken (Fälschungen); in: Moschkau, A., Zur Geschichte der Philatelie.
'I'ramburg's Erloen: Obersicht aller bekanmten von 1849 bis 1862 emittirten FrancoMarken, welche in gröBerer Anzahl vorräthig und unter der Ziffer ${ }^{\circ} \mathrm{Ab} 1863^{*}$ bei Tram. burg's Frben. Hamburg: Tramburgs Frben [1863]. 8 S. $4^{\circ}$.

[Das Jahr 1849 ist irrtüulich statt $1840^{4}$ aufge-

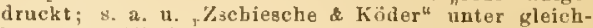
lantendem Titel gedr ]

'Trillich, IIeinrich: Das Wasserzoichen und seine Erkeunung in Postwertzeichen. Preisgelirönte Arbeit. Eigentum des Vereins für Briefmarkenkunde zu Magdeburg. Magdeburg: Verein f. Brietmarkenkunde 1890. 8S. $8^{\circ}$.

Tsingtau. Friedemann, Die Aushülfsmarken von T'singtau und ihre Fälschungen .... Leipzig Iy03.

Turin. Philatelistische Ausstellung unter dem Patronat des Ministeriums für Post- und Telegraphenwesen. Turin, 16. bis 30. Mai 1898. 6 S. $8^{\circ}$

[Prospekt der Ausstellungoleitung in deutscher sprache.]

Tiirkei. Andersch, M.: Dio deutsche Post in der Türkei, in Clina und in Marokko ... Berlin 1912.

- Antoniades, J. T.: Katalog über orientalische Postwertzeichen. Constantinopel 1892.

- Hruby, R.: Katalog aller türkischen, ostrumelischen, bulgarischen und russischen Levantepostmarken, Karten $u$. Couverts mit Preisen. Constantinopel 1884-1891.

- Meyer, Ferd: Katalog der Postwerthzeichen des ottomanischen Kaiserthums.

- Raditch \& Co., G.: Katalog ïber Orientalische Postwerthzeichen. Constantinopel 1888.

- Zarmikian, G.: Engros Endetail. PreisCourant iiber Orientalische Briefmarken. Varna (Bulgarien) I889.

Tybring, Julius: 1887. Preisliste über skandinavische Briefmarken, zu beziehen von J.T. Kopenbagen 1887. $16 \mathrm{~S} .8^{\circ}$.

Typensammelu, Üter Systematisches s. Caspart, Rudolf.

Ueherall, Hans: Die Panama-Gedenkmarken der Vereinigten Slaten von Amerika. Son. derdr. aus der Deutschen Briefm.-Zeitung H. 3. Jg. 1917. Chemnitz: Selbstverlag 1917. 9 S. m. Alb. $8^{\circ}$.

Ubersicht der in del Zeitungsliteratur der Jahre 1894 bis 1896 über die Postwertzeichen der Deutschen Staaten enthaltenen Aufsätze etc. s. Rommel, Otto.

Uhlich, Willian s. Kohl. Paul: P. K.'s illustriprter Freimarken-Katalog "Englische Kolonien" Abt. 3.

Ulex, 0scar: Ausführlicher Preis-Catalog aller seit 1840 bis jetzt emittierten Briefmarken. Hamburg: Selbstverlag 1872. 37 S. u. 1 Taf. m. Abh. $8^{\circ}$.

Ungebraucht. Walser, Hugo: Las Sammeln "ungebrauchter" Marken. Zürich 1916.

Unter dem Zeichen des Verkehrs. s. [Billig, B.]

Uruguay. Hanciau, Louis: Die Diligencias von Uruguay. Berlin 1913. 
Vallemeeun des Philatelisten, s. Finke, Geory.

Yodel, M.: Dr. M. Vedel, Kopenhagen K. Anerkannt beste Bezugsquelle für ernsthafte 11. fortgeschrittene Sammler. [Spezialofferte $\mathrm{Nr}$. I] liopenhagen [1884] $6 \mathrm{~S} .4^{\circ}$.

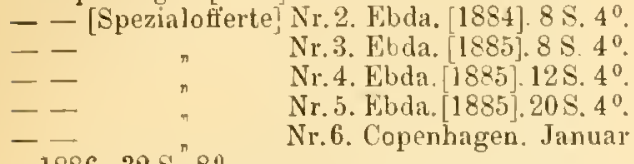
1886. 32 S. $8^{\circ}$.

- - Spezialofferte] Nr. 7. Ebda. Februar 1887

IV, 132 S. $8^{\circ}$. [Sonderausg. auf dickem Papier.]

- - [Spezialofferte] Nr. 8. Fbda. Novbr, 1889. $53 \mathrm{~S} .8^{\circ}$.

- - [Spezia]offerte] Nr. 9. Kbda. Närz 1891. 50 S. $8^{\circ}$.

Verband der Sammler rou Ibstempelnngeu: Verzeichnis der in Gruppen auftretenden Poststempel der deutschen Einzelstaaten u. Kolonien. 8. Glasewald.

Yeredarius, 0.: Das Buch von der Weltpost. Fintwickelung u. Wirken der Post u. Tele graphie im Weltverkehr. Berlin: Herm. J. INeidinger 1885. 400 S. m. 2 Karten u. Abb. im 'Text, n. 20 'Taf. 4\%".

- Dritte, durchges. Autl. (Neuntes Tausend.) Ebda. 1894. 367 S. n. 14 Taf.u Abb. i. Text. $4^{0}$. [In dieser Ausg, fehlt u, a ach die Postwertzeichentafel.]

Verein, Berliner, für Privatpost-Wertzeichen. s. Wagner, F.

Vereine für Hriefmarkensammler, s unter dem entsprechenden Namen u. Sitz.

- s. a. u. Adressbiucher und Verzeichnis der Philatelisten -Vereine.

Vereinigte Staateu yon Nordamerikid. Albrecht and Witt, New York: Suchen zu den hieriu aufgeführten Preisen Vereinigte StaatenMarken zu kaufen. New York [1892].

Kloss, Paul: Vereinigte Staaten von NordAmerika. Kine Convert-u. Streifband-Anfstellung. Leipzig 1880.

- Kohl, Paul: Illustr. Freimarkin-liatalog, "Englische Kolonien“, Abt. 1 "Nordamerika" Chemnitz 1898.

- Probedrucke, Marken mit Specimen, sowie Essais der Vereinigton Staaten voll Amerika. Chemnitz 1911.

- Sellschopp. Wilhelm: Die Express-Gesellschaften des westl. Nordamerika u. ihre Wertzeichen. Berlin u. Dresden 1907. Leipzig 1908.

- - Western Franks, Freicouverts der Anerican Express-Compagnien. Hamburg 1907.

- Ueberall, Hans: Die Panama-Gedenkmarken der Vereinigten Staaten von Amerika. Clem. nitz 1917.

Vereinsfrage. s. lsakovics, A. v.

Vereinsgeschichte des Philatelisten-Vereins "Rund um Berlin“ 1901-1905. Zsgest. aus Anlaß des 5 jährigen Stiftungstestes vom Vereins-Vorstand. Berlin: Selbstverl. 1906. 8 S. $8^{\circ}$.
Yereinswesen. König, Luclwig: Über Vereinswesen. Vortrag, gehalten auf dem XIll. Deutschen Philatelistentag. Berlin 1901.

Verkelur, Der, mit der Feldpost. Ein prak tisches Ililfstuch mit ausgefüllten FormularII ustern, Portotarifen n. Versendungs-Vorschriften. Nach amtlichem IIaterial bearb. Giessen: von Nünchow'sche Hof- $n$. Universitäts-Druckerei. 1914. $8 \mathrm{~S} .8^{\circ}$.

Verkehrswerthzeichen. Finiges zur Geschichte der Verkehrswerthzeichen; in: Jeran, 0 . Wegwriser tür Verkebrswerthzeiclıen-Sammler. (Philatelisten-Fibel.)

Vorsicht beim Ankauf von Terkehrswerth. zeichen; in: Jeran, O.: Wegweiser f. Verkehrswertbzeiches-Sammler. (PhilatelistenFibel.)

Veröllentlichungen, Philatelistische, in Tageszeitungen und Familienjournalen. s. König, Ludwig.

Verslcigerungs.Kalaloge. Köhler, Heinrich [Katalog der] Briefmarken-Auktion Nr. 14 Stückweise Versteigerung einer P'rachtsammlung im Küunstlerhaus Berlin W., Bellevuestr.?. Versteigerungatage: 12. bis 14. Sept. 1917. Berlin: Heinrich liöhler 1917. 67 S. m. Abb. i. Text und 20 Kunstdr.-Taf. $8^{\circ}$.

[Die vorhergehenden Kataloge Nr. 1-11 B. unter Auktions-Kataloge"; Nr. 12 und 13 unter, Köhler, Heinrich".]

- - Nr. 15. Stückweise Versteigerung einer wundervolien Pracht-Europasammlung im Künstlerhaus Berlin W., Bellevuestr. 3. Versteigerungstage: 12. bis 15. November 1917. Fbda. 1917. $76 \mathrm{~S} . \mathrm{m} \mathrm{Abb}$, i. Text u. 28 Kunstdr.-Taf. $8^{0}$.

- _ - Nr. 16. Stückweise Versteigerung einer Prachtsammlung im Künstlerhaus Berlin W., Bellevuestr. 3. Versteigerungstage: 21. bis 24. Januar 1918. Ebda. 1917. $110 \mathrm{~S}$. ra Abb. i. Text u. 20 Kunstdr.-Taf. $8^{\circ}$.

- Maier, M. Kurt: [Katalog der] BriefmarkenVersteigerung [im Künstlerbaus - Bellevuetr. 3, Berlin W. Versteigerungstage: 22. -24 . X. 1917. [Nebst:] lllustrationsbeilage [auf Kunstdruckpapier]. Berlin: M. Kurt Maier 1917. 64 S. m. Abb. i. 'Text u. 21 Taf. $8^{\circ}$.

- - - Versteigerungstage: 3.-5.Januar 1918. [Nebst:] fllustration-beilage [auf Kunstdruckpapier]. Ebda. 19I8. 56 S. m. Alb. i. Text u. 15 Taf. $4^{0}$.

- - Versteigerungstage: 25 -23. Februar 1918. [Nebst:] Il] ustrationsbeilage [auf Kunstdruckpapier]. Ebda. 1918. 96 S. m. Abb. i. Text u. 22 Taf. 80 .

(Sammlung ans dem liesitze eines unserer bedeuteudsten rheinischen Plilatelisten.)

[Die früheren Kataloge s. unter Auktiongliataloge i1. Briefmarken Versteigerungen".]

Marken- und Ganzsachenlnus G. m. b. H. Berlin. Verzeichnis der ersten BriefmarkenKriegs-lVersteigerung Zum Verkauf gelangen zwei prachtrolle Sammlungen, davon eine wit der goldenen Medaille prämiiert, sowie einige andere Stïcke im kiünstlerhaus Berlin W., 
Bellevuestr. 3. Verkaufstage 22,-24, Janual 1917. [Nebst:] lllustrationsbeilage mit 20 'Taf. auf Kunstdruckpapier. Berlin: Marken-und Ganzsachenhaus G. m. b. H. 1917. $82 \mathrm{~S} 4^{\circ}$.

[8. a. u. Marken- U. Gauzsachenhaus G. m. b. H. Berlin [Katalog der] Briefmarken-Versteigerungen v 22.-24. Januar 1917, wounit vorliegeuder Titel gleichbcdeutend jst.]

- - (Verzeichnis der) zweiteu Kriegsversteigerung im Künstlerhaus Berlin W., Bellevuestrabe 3. Eine Auslese edelster Stücke von Alteutschland u Furopa. Versteigerungstage: [Ursprünglich 27 -29. Juni 1917, sodann aber verlegt auf]: 9.-11. Inli 1917. Ebda. 1917. 103 S. m. Abb. i. Text auf Kunstdr.-Papier. $4^{\circ}$.

- - (Verzeichnis der) dritten Kripgsversteigerung, 17.-20. Dezpmber 1917 im Künstlerhaus Berlin IV., Bollevuestr. 3. Eine Fülle Edelstücke aller Länder. Beschrieben und geschätzt vou hiudolf Siegel. Fbda. 1917. $197 \mathrm{~S}$ m. Abb. i. 'lext auf Kunstdr.-Papier. $4^{\circ}$.

- s. a. u ,Auktions-Kataloge u. BriefmarkenVersteigerungen".

Verzeiehniss der aus dem Leutzsch'schen Concurse herrührenden, vom Deutschen Philatelisten-Verband erworbenen Fohl'sehen Fïlschungen und dazugehörigem Druckmaterial. [Sonderabdr. aus der , Mitteldeutschen Philatelisten-Zeitung" 1898]. Gössnitz, S.-A.: A. E. Glasewald 1898. 2 S. m. 12 Abb. im Text $\mathbf{~}$. 11 Fälschungs-Beigaben, 40.

- der Mühlradstempel nach Orten und Nummern. s. Stündt, Johann Wilheln: Die Abstempelungen auf den Postwertzeichen von Bayern.

- s. a. Stenger, Frich: Die Mühlradstempel von Bayern.

- s. a. Stenger, Erich u. Ludwig Sauter: Die Postablagen-, Landpostboten- u. Posthilfsstellen-Stempel von Bayern.

- der in Gruppen auftretenden Nummern-, Orts- etc. Stempel der deutschen Hinzelstaaten u. Kolonien. s. Glasewald, A. F.

- der Nummern-u. Orts-Abstempelungen von baden. s. Glasewald, A. E.: Baden-StempelVerzeichnis.

- von Thurn u. Taxis. s. Taxis-Taxen.

- - - s. a. Ton, Max: Die 'Thurn v. Taxisschen Postfreimarken.

- der Philatelisten-Vereine und der philatelistischon Literatur; in: Jeran, O.: Wegwejser für Verk fhrswerthzeichen-Sammler. (Hhilatplisten-Fibel.)

- über die Postwertzeichen von Schweden. Marken. Stockholm: Sveriges FilatelistFörening 1906. 30 S. m. 114. Abl, 8?.

- Dass. Ganzsachen. Stockholm: Sreriges Filatelist-Förening 1907.

- Dass. Marken [Teil 1]. Ebda. 1911. $38 \mathrm{~S} .8^{\circ}$

- der z. Z. bestehenden deutschen PrivatpostUnternehmen. [Gössnitz, S.-A.: A. F. Glasewald 1890.] $2 \mathrm{~S}$. $8^{\circ}$. [Einseitig bed..
Verzeichnis der wichtigsten pluilatelistischen Werke u. Al)handlungen des ln- u. Auslandes mit Ausschluss der Zeitungsliteratur. s. Rommel, Utto.

- der in jhilatelistischen Zeitschriften ... euthaltenen Fachartikel $u$.der ... erschienenen Handbücher. s. 'T'halmann, Friedrich Wilhelm.

Verzijl, J'an: lllustrierter T'elegraphennarken. Katalog. Louvain u. (Berlin: Philipp liosack in Komm.) 1912, $118 \mathrm{~S} .8^{\circ}$.

Vieenz, Ernst: Gibt es amtliche Neudrucke u. Probedrucke von Hamburger Steindruckmarken? Vortrag. s. Drutscher Philatelistentag; in: Stenograph. Bericht des XIX. Deutschen Philatelisten-Tages ... zu Hamburg 1907.

[Auch als Sooderbeilate zu "Der Philatelist" und "Philatelist. Miscellen" 1907.]

- Die Kunst im Bilde der Briefmarken. Vortrag. s. Deutscher Philatelistentag; in: Die Vorträge vow XXIl. Deutschen Philatelistentage in Kiel. Leipzig 1910.

Vigcner, 1.: Über Reinigen u. linkleben der Varken; in: lsakovics, A. v.: Interuationales Tausch-, Adress- u. Hülfsbu h f. den Tauschverkehr. 'T'. 2.

Vilicl. Frity. s. Fühtmann's I'osthandluch.

Vineta-I'rovisorium, s. Dister, G.

Voglliudisclıer PlilateIisten-Verlanul. s. Lobenstein (heuss j. L.).

Vollmers d Eekardt: Preiscourant von Briefmarken aller Lïnder. Bremen 1873. 8\%.

Vou Jehrling his zum Meister. Briefe von Onkel Oskal an seinen Neften Wilhelm, der lernen will, wie man richtig Briefmarken sammelt. Berlin: Philipp Kosack 0.I. (1911). $55 \mathrm{~S} .8^{\circ}$.

Vordruckalbum. Reform des Vordruckalbums. s. Haas, Theodor; Klenze, Heinrich von.

Torzugs-ingebot seltener Marken. s. Kolı], Paul.

Wagner, Fr.: Die Privatpost-Werlzej'hen, H.l. ${ }_{n}$ Heidelberg. Berlin-Friedenau: BerlinerTerpin für Privatpost-Wertzeichen 1912. 27 S. 8".

- s. a. Glasewald, A.E., O. Sattler \& Fr.Wagner: Handbuch der deutschen Privatpostzeichen.

Walser. Ilugo: Das Sammeln ungebrauchter. Briefnarken. Zürich: Artist. Institut Orell Füss]i 1916. 32 ร. $8^{\circ}$.

Waulkalender. s. Heitmann, Ernst.

Warseliau. s. Stadtpost Warschau.

Was dann? s. Sachse, Paul.

- muss der Briefmarkensammler wissen? s. S'chidlof, Berthold.

Wasvermanu, 0, u. Heinrich Fräikel: Rumïnien, Ausgab+n 1866-1872. (Sonderds: ius der Festschrift zur Feier des zehnjïlur. Bestehens des B. Ph. Iil. Berlin 1898. $30 \mathrm{~S}$. m. 3 Lichtdr.-Taf. $8^{\circ}$.

- s. a. Berlin. (Berliner Philatelisten-Klub.) lestschrift z. Feier des zehnjailur. Bestehens des Li, Ph.-lil. 
Wasserzeicheu. Moschkau, Alfed: 1)ie Wasserzeichen auf den seit 1818 bis dato enittienten Briefmarken. Dresden 1871 u. 1872.

_ - 3. Aufl. u. d. T.: Geschichte der Briefmarken u. der Philatelie (Briefmarkenkunde). Nebst einer Beschreibuug aller bekannten Wasserzeichen auf Briefmarken. L,pipzig 1878.

- - 4. Aufl. u. d. 'l':: Die Wasserzeichen auf Briefmarken. lueipzig 1880.

- Stenger, Frich: Die objelitive Feststellung Wasserzeichen in schwierigen Fällen. Berlin 1914.

- Trillich, Heinrich: Das Wasserzeichen und seine Krkenmung im Postwertzeichen. Magdeburg 1890.

- Was gilt von Wasserzeichen? in: Jeran, U.: Wegweiser f. Verkehrswerthzeichen-Sammlel (1'hilatelisten-Fibel).

Weber, 1.: Postgebührentabelle. 2. verb. Aut. (1 Bl, $44,5><50 \mathrm{~cm}$.) 'Trier: Śelbstverl. (Paulinus Diuckerei) 1916.

V'ecliselstempelmarke, Die. s. Hartung, H.

Veegmanu, E.: Stellungnahme gegen den Hamilton'schen Vertrag mit den Centralamerikanischen Republiken. s. Deutscher Pbilatelistentag, Antrag 1; in: Bericht über den... Il. Deutschen Philatelisten-T'ag zu Frankfurt a/M. 1890.

Wegweiser für Markensammler. s. Schanfuss ¿ Stolpe.

- für T'erkelı'swertzeichen-Sammler. s.Jeran, O.

Wehner, Fr.: Über halbiert gebrauchte Marken des Deutschen Reichspostgebiets. Vortrag. (Sonderabdr. a. "Germania-Berichte" v. 25. Novbr. 1900.) Hamburg: [Sel'stverl. 1900]. 4 S. u. 1 Taf. m. Abb. $4^{0}$.

Weidenhagen, Robert: Die Ursachen ïber den Rückgang in der Philatelie. Vortrag, geh. am 25. Februar 1898 im Hamburg Altonaer. Briefmarken-Sammler-Verein. (Sonilerabdr. a. d. Vereins-Mitteilungen v. 16. Mai 1898.) [Hamburg: Selbstverl. 1898.] 4 S. 8".

Weissenfels (Briefmarken Sammlerverein $\langle e . V$. Weissenfels). Fübrer dureh die 11. Briefmarken Ausstellung 14. Novbr. 1909. Weiss+nfels: [Ausstellungs-Komitee] 1909. $12 \mathrm{S.}$.

Weithase, Hugo: Geschichte des Wreltpost. vereins. Vou der rechts u. staatswissenschaftl. Fakultä.t der Kaiser- IVilhelms-Universität zu Strassburg (Elsass) preisgekrönt. Strasshurg: J. H. Ed. Heitz 1893. 111, 88 \$. $8^{\circ}$.

- 2. erheblich vermehrte u. verbess. Aut. Eboda. 1895. 1848. 80 .

Welt - Briefmarken - Katalog. (Beigabe zu Schwanebergers Briefmarken-Album, KaistalAusg.). Leipzig: Sclıaufuss it Stolpe 1914.

Weltpost. Beck, Otto: Weltpost, Reichspost, Maiuzer Post. Mainz 1909.

- Jacobsen, Niels: Dif Zeitbücher tel" Weltpost. Leipzig 1892.

Schweiger Lerchenfeld, A. Frhr. von: Jas neue buch von der Weltpost. IVien 1901.
Weltpost. Stephan, Wrast I einrich Wilhelm von: Veltpost u. luftschifffahrt. Berlin 1874.

- Terularius, O.: Das Buch ron der Weltpost. berlin 1885 u. 1894.

Weltpostrerein. Haass, l': Der Weltpostverein. Lulwigsburg 1893.

- Jung, .I.: Der Weltpostverein u. der Wiener lostkongress. loiprig 1892.

- Der Weltpostrerein u. sein Einfluss auf den Weltverkebr und dio Weltwirtschaft. Strassburg 1903.

- Katscher, L.: Der Weltpostverein. Gautzsch b. Leipzig 1909.

- Heyer, A.: Die deutsche l'ost im Wellpostverein u. im Wechselverkchr. Berlin 1901 u. 1908 .

- Schröter, Carl: Der Weltpostverein. liern 1900.

- Weithase, H : Geschichte des Weltpost. vereins. StraBlurg 1893 u. 1895.

Weltverkelurskartes. Opitz C. u. I'aul Lederer: Lücke's Ländel- und Weltverkehrskarte für Postwertzeichen-Sammler.

Wende, (ieorg (Pseud. Kaufmann): Biographie in: Jeran. O.: Wegweiser für Verkehrswert zeichen-Sammler.

- s. a. in: Isakovies, A. r.: Internationales 'lausch, Adress- u. Hülfsbuch f. d. 'I'auschverkebr' T.?.

Wemden. Hirschheydt, A. v.: Ein neuer Śchluss zum Artikel "Wenden". Berlin 1908.

- Svenson, W.: 'Typen u. Abarten der Wendenschen Originalmarken, offiziellen Neudrucke u. Fälschungen. Berlin 1907.

Wenzel, Ernst: Philatelistisches Nachschlagebuch. Enthaltend ein Verzeichniss der Adressen aller Vereine, von Sammlern und Händlern, die Preise seltener Briefmarken, eine Auswahl fachwissenschaftlicher Aufsätze und eine vollständige Uebersicht der Fachliteratur. Coepenick-Berlin: Selbstverlag [u. Leipzig: Lietr. Senf in Komm.] 1886. 160 $\% 8^{\circ}$.

Die fachwissenschaftlichen Beiträye lieforter Dr. H. Brendicke, Berlin und Dr A. Moschkau, Oybin Von ersterem enthält das Buch: Ist die Plilatelie Wissensebaft oder nur Manie? und eine Skizze über Fiuher nad Firclerer des fostwesens. Lotztgenannter schrieb "feschichte der Postwerthzeichen und ih her die Entwickelung der Briefmarkenkuude. Weiler entbilt das Buch drei biographische Skizzen der Philatelisten Mosclikau, Kloss und Friedl.\}

Wer sammelt eigentlich Briefmarken? in: Moschkau, A., Geschichte der Philatelie.

Veruigg, Franz: Verzeichnis jener Adressen, welche für jerlen reellen Philatelisten wissens wert sind. Wien: [Selbstrerl.| 1896. 56 S. 8".

- Nachtr. 1. Fbda. 1896. 16 S. $8^{\circ}$.

Wrumek is Co., H.: Catalog aller seit 1818 bis April 1874 erschienenen Brimfnaken, Couverts u. Karten, bearbeitet von $\mathrm{D}_{\mathrm{r}}$. A. Moschkau. Leipzig: Werninct: \& $\mathrm{Co}$. 1874. 192 S. m. 4 Taf, farb. Ahl, 4".

Wertmarken, Die. s. Isaluand, J'anl. 
Wessel, lt.: Engros Preisliste Nr. l über Briefetc.-Marken. Bremen: Selbstverl. 1877.4S. $4^{\circ}$.

Westindien. Kobl, Paul: Illustr. FreimarkenKatalog "Englische Kolonien". Abt. 1: W'stindien. Chemuitz 1898.

Wesloloy, William Ames Searborongh: Bleuté par la goinme [nebst deutscher Übersetzung]: Vom Gummi gebläut. s. Berlin (Berliner Philatelisten-Klub) in: Festschrift zur Feier des zehnjähr. Bestehens des B. Ph.-KI. Berlin 1898.

Wie ergänze ich mein Album? Leipzig: C. F. Lüclie. o. J. (1906) 32 S. $8^{\circ}$.

[Bildet den 1. Jahrg, von Lüicke"s Jahrbuch für Briefmarken-Sammler.]

- es ist, wie es nicht ist und wie es doch sein könnte! s. Genth, F. (Vortrag geh. auf dem XXV. Deutschen Philatelisten-Tag zu Hannover 1913.)

Wien (Internationales Postwerthzeichen-Mluseum). Isakovirs, A. ron: Das Internationale Postwerthzeichen-Museum in Wien iu: Isakovics, A. v.: Internationales Tausch-, Adressn. Hült'sbuch für den Tausclıverkehr. T. 2.

- Katalog der I. Internationalen Postwertzeichen-Ausstellung in Wien vom 20. Apri] bis 4. Mai 1890. Wien: [Ausstellungskom.| 1890. $36 \mathrm{~S} .8^{\circ}$.

- s. a. Friedl \& Baum u. Koch, Geors.

- (Weltausstellung.) Die Briefmarken auf der Wiener Weltausstellung; in: Moschkau, A., Zur Geschichte der Philatelie.

Wilde, H.: Lübecks Postwertzeichen. Vortrag, geh. im Verein f. Freunde der Briefmarkenkunde zu lübeck am 24 Jan. 1889. (Sonderabdr. a. d. „1ll. Briefmarken-Joumal 1889 Nr. 7.) Leipzig: Gebr. Senf $1 \& 89$. 6 S. m. Abb. $8^{\circ}$.

Wildt, Arthur: Preisliste. Krakau, Oktober 1866. 11 S. $4^{\circ}$.

- - [Neue Ausg.] Ebda. Januar 1867, 11 S. $4^{0}$.

- - [Neue Ausg.] Eibda. August 1867.

Willelm, Prinz, von Preusson. Fin fürstlicher Philatelist; in: Moschkau, A., Zur Geschichte der Philatelie.

Willelı, J.: Biographie in: Jeran, O.: Wegweiser für Verkehrswertzeichen-Sammler.

Willadt, Car]: Jsland speziell ] Gildi. [Bemerkenswerte kurze Ausführungen zu d’m ursprünglich vorgesebenen aber nicht gehaltenen Vortrag des Dr. Bergmann, Hannover]. s. Deutscher Philatelistertag; in: Stenograpli. Bericht ï. d. XVI. Deutschen PhilatelistenTag . . . in Leipzig (u. Halle a. S.) 1904.

- Über liatalogpreise! Vortrag. s. Deutscher Philatelistentag; in: Stenograph. Bericht üler den XVIJl. Deutschen Philatelistentag ... in Nürnberg 1906.
Willadt, Carl: Jager. Preisliste $\mathrm{Nr} .1$ der Firma C. W. Pforzheim (Baden) 1896. ... S. $8^{\circ}\left(16^{\circ}\right)$. - Nr. 2 ... Ebda. 1897. 255 S. $8^{\circ}\left(16^{\circ}\right)$. - Nr. 3. . . Ebda. 1898. 256 S. $8^{\circ}\left(16^{\circ}\right)$. — - Nr.4.] . . Kibda. 1899. 296 S. $8^{\circ}\left(16^{\circ}\right)$. - Nr. 5. . . Ebda. 1900. 287 S. $8^{\circ}\left(16^{\circ}\right)$.

- Nicht-Katalogisiertes aus dem Gebiete der altdeutschen Staaten. Vortrag s. Deutscher Philatelistentag; in: Stenograph. Bericht ü. d IV111. Deutschen Philatelistentag ... in Nürnberg 1906.

Willad1, Car] \& Co.: Lager Preisbuch [Nr.6] besonder's hervorragender Objekte, alle Länder umfassend. Pforzheim (Baden): Carl Willadt \& Co. 1902. 252 S. m. Abb. $8^{\circ}$.

- - Nr.7. 1902,03. Ebda. 1902. 160S. $8^{\circ}\left(16^{\circ}\right)$. - Nr.8. 1904/05. Ełıda. 1904. 152S. $8^{\circ}\left(16^{\circ}\right)$. - Nr.9. 1905/C6. Ebda 1905. 168S. $8^{\circ}\left(16^{\circ}\right)$. - - Nr.10.1906/07. Ebda. 1906. 168S. $8^{\circ}\left(16^{\circ}\right)$

- Europa Spezial-Preisbuch Nr. 11. 1907. Ebda. 1907. 112 S. m. Abb. $8^{\circ}$.

- Nr. 12. 1908. Ebda. 1908. 112 S. m. Abb. $8^{\circ}$.

- Nr 13. 1909. Ebda. 1909. 116 \& m. $\mathrm{Abb}, \mathrm{S}^{\circ}$.

- Nr. 14. 1910. Kbda, 1910. 116 s. m. Abb. $8^{\circ}$.

- Nr. 15. 1911. Ebda. 1911. 120 S. m. Abb. $8^{\circ}$.

- Nr. 16. 1912-1913. Fbda. 120 s.m. Abb. $8^{\circ}$.

- Nr. 17. 1913-1914 [nebst Beilagren]: Hande] u. Verkehr in der Philatelis im Jahre 1912-1913 u. Sonderangebot für Überseemarken. Ebda. 1913. 128 S. m. Abb. $8^{\circ}$ +4 S. $4^{\circ}+65$ S. $8^{\circ}$.

- Illustr. Preisliste über Marken der Kriegsausgaben. Ebda. 1915. ... S. $8^{\circ}$.

Wiltz, llermaun: Die Feldpostdienstordnung nebst Ausführungsbestimmungen in gedrängter Darstellung. Anh.: Wiederholung in Frage u Antwort. Schwerin i. Mecklenb.: Ludwig Davids 1911. IV, $32 \mathrm{~S} .8^{\circ}$.

Windratl, W.: Preisliste über Briefmarken anglischer Kolonien in Asien u. Ostafrika. Zürich: Selbstverl. 1911. .... S. $8^{\circ}$. [Auch: Basel: Gustav Schmidt in Komm.

— - Nachtr. Ebda. 1912. ... S. 8\%.

Wissenschaftiche Gruppe, Zweck und Ziele. s. Pauli, Emil.

Wissenswerles, Allerlei, iiber Europa-Marken. s. Brausewetter, Rudolf.

Withalm, Hanus: Der Briefmarken-Samuler. Leipzig: Hachmeister \& Thal 1910. 51 S. m. 41 Abb. $8^{\circ}$. (Lehrneister-Bibliothel Nr. $30,51$.

— - 2. Aufl. Ebda. 1917. 51 s.m. 38 Abb. 80.

Wit1riseh, Max: Jahrbuch der Philatelie 1881. Eine kurze Statistik dieser jungen Wissenschaft Borna: H. Schumann 1881. $98 \mathrm{~S} .8^{\circ}$.

Wohi川 soll das führen? s. Scliardey, l'aul. (Vortrag, geh. auf dem XVIII. Deutschen Philatelistentag in Nürnberg 1906.) 
Wohiu treiben wir: s. Sellschopp, Wilhelus, (Vortrag, geh. am XII. Internationalen Briefmarken-Händlertag in Berlin 1913.)

Woprz, Ritter IIans rou: Über die Neudrucke der Marken von isterreich und der l,ombardei Vortrag s. Deutscher Philatelistentag; in: Stenograph. Bericht des X.III. Deutschen Philatelistentages... zu Wien 1911.

Wiilbern, Arthur: Originale und Nendrucke von Helgoland. Eine Mlarkenstudie. Hamburg: Selbstverl. (1906). 64 S. m. Abb. 8".

- Die Postwertzeicben Helgolands mit beson. derer Berijcksichtigung der Falsifikate sowie der falschen Abstempelungen nebst einem Abriss der Postgeschichte Helgolands. Bre. men: August Narbes 1892. $32 \mathrm{~S} . \mathrm{m}$. Abb. $8^{\circ}$.

- Illustrierter Preis-Katalog der Telegraphenund Stempelmarken Deutscher staaten und Kolonien. 2. gänzlich neubearb. Aufl. Hamburg: Arthur Wülbern 1912. 62 S. m. Abb. $8^{0}$. Frste Autl. s. u. d. 'T. Berger, H]

- Bd. 2. Oesterreich-Ungarn und Balkanstaaten. 3. Berger, H.

Wiirttembery. Herrmann, Adolf: Druckdaten der Postkarten von Württemberg. Berlin 1913.

- Lindenberg, Carl: Die Briefumschläge von Württemberg. Berlin 1895.

- Moens. J. B.: Die Postmarken von Wiurttemberg. Berlin 1881.

- Post-Tarif, Neuer, ah 1. August 1916 mit besonderer Berücksichtigung des wïrtt. Postverkehrs. Stuttgart [19i6].

- Sommerfeldt, Willy: Die als lienstformulare verwendeten ïberdruckten Kreuzer-Postkarten von Württemberg. Berlin 1913.

Wuttig, (r.: İbersicht aller bekannten von 1840 bis August 1863 ausgegebenen FrancoMarken (Timbres-Poste - Postage Stamps), welche meistens in grösserer Anzahl vorräthig u zu den beigesetzten Preisen zu haben sind bei G. W. Neuer revidirter Abdruck. Leipzig: Selbstverl. 1863. 30 \. $8^{\circ}\left(16^{\circ}\right)$.

- Übersicht aller seit 1840 bis Juli 1864 ausgegebenen Franco-Marken. . . Dritter, revidirter $\mathrm{u}$. ergänzter Abdruck. Kbda. 1864 34 S. $8^{\circ}\left(16^{\circ}\right)$.

I $1 / 4$ s. Friedricb, Rudolf.

Zahlenzeichen, Orientalische. s. Kausch, Oskar.

Zarmikian, G.: En gros. Fn détail. PreisCourant über Orientalische Briefmarken ... Varna (Bulgarien): Selbstverl. 1889. $24 \mathrm{~S}$. $8^{\circ}\left(16^{\circ}\right)$.

Zechmeyer, Feorg: Der Briefmarkenhandel vor den Breslauer Gerichten u. Zechmeyer contra Dr Joseph wegen Releidigung Niirnberg: $1888.23 \mathrm{~S} .8^{0}$.

Zeit, Die neue, und die alte deutsche Reichspost. Hagen i.W.: Risel \& Co. 1892. 118 S. 8\%.

Zeitbiicher, Die, der Weltpost. s. Jacobsen, N.
\%eitungs-Katalog, Internationaler t'hilatelistischer, 1910. (Mit Geleitwort von lir. Ottu Rommel) Leipzig: Malke (1909). 47 S. 8 .

Mentralorgan fiir die Giesamtinteressen der Briefmarkenkunde. Gründung eines solchen. s. Deutscher Philatelistentag. Lericht ü. d. am 9. u. 10. August 1890 abgehaltenen J. D. Ph.-T. zu Frankfutt a. M. Antrag 3.

Kennig, Karl: Die Kommune-Post von 1871 s. Berlin (Berliner Philatelisten-Klub) in: Festschrift nur Feier des 25 jïhr. Bestehens des B. Ph-K. Berlin 1913.

Zeulenrotia s. Lobenstein (Reuss j. L.) Katalog der Postwertzeichen-Ausstellung.

Z,chiesche, Alwin: Katalog über alle seit 1840 bis Januar 1868 ausgegebenen Briefmarken mit beigedruckten Verkaufspreisen Leipzig: Reinherz Zschiesche 1868. IV, $32 \mathrm{~S} .8^{\circ}$. _- Nachtr. Leipzig: Alwin Zschiesche [1868] 2 S. $8^{\circ}$.

- Dritte Aufl. Ebda. 1869. IV, 36 S. $8^{\circ}$.

- - Vierte Aut. Ebda. 1870. IV, 38 S. $8^{\circ}$.

- - Nachtr. Ebda. [1871.] 8 S. $8^{\circ}$.

- - Fünfte Anfl.] Ebda. 1872. 45 S. $8^{\circ}$.

- - Nachtr. Fbda. 1872.] 4 S. $8^{\circ}$.

— - Sechste Aut.] Ebda. 1873. 51 S. $8^{0}$.

- - Siebente Aufl.] Fbda. 1875. 56 S. 80.

- - Nachtr. Fuda. [1876.] 13 S. 8".

- - Achte Aufl. Ebda. 1877. 66 S. 80.

[Auch mit gleichloutendem Text unter dem Namen Reiuherz Zschiesche" gedr.]

- - [Neunte Aufl.] 2 Teile. Elida. 1880. $8^{\circ}$. T. 1. $64 \mathrm{~S}$. T. II $21 \mathrm{~S}$.

- Zehnte AuQ.] Naumburg a. S.: Alwin Zschiesche [1883],96 S. $8^{\circ}$.

[A uch mit dem Uimscblage "Literarischeo Museum" Katalog über alle geit 1810 usw, auggegebenen Brief marken, jedoch textlich unverändertem Inhalt gedruckt.!

- - Nachtr. Ehda. [1884.] 12 S. $8^{\circ}$.

- - Elfte Aufl] Ebda. [1885.] III, 112 S. $8^{\circ}$.

- - Nachtr. Fbda. [1887.] 16 S. $8^{\circ}$.

_- [Zwölfte Au甘.] Fhda. [1888.] IV, 100 S. $8^{\circ}$. - - - Nachtr. 1888/89. Fbda [1889] 9 S. 8".

- Katalog über alle bis 1896 ausgegebenen Briefmarken von Furopa von A. Z., etabliert 1867. Ebda. [1896.] 50 S. $8^{\circ}$.

- Katalog sämtlicher bis 1891 ausgegebenen Couverts, Postkarten und Kreuzlandstreifen der deutschen Staaten. Naumburg a. S.: Alwin Zschiesche 1891. 16 S. $8^{\circ}$.

- Probebogen des gegen Finde Oktober d. J. erscheinenden nach der Alfred Mloschkauschen Sammlung bearb. Kataloges aller existierenden Briefmarken. Leipzig: Alwin Zschiesche 1870. 16 S. $8^{\circ}$.

[Nur bis Costa Rica gedr.]

- s. a. Dautb, H. J., Manco-Listen u. Pilzpcker, O., Preisbuch Nr. 1.

\%sefiesche. Reiuher\%: Briefmarken-Preiscourant. Leipzig: [Selbstverl. [1867]. 8 S. $8^{\circ}$.

- s. a. Zschiesche, Alwin: Katalog über alle seit 1840 bis Januar 1868 ausgegebenen Briefmarken. Leipzig 1868.

- Katalog [Achte Auf.] Euda. 1877. 
Zschieseled köler: von 1849-1862 emittirten lianco-Marken. Leipeig: 'Lschiesche \& Köder [1862]. 10 S. 8".

[Irrtumlich 1849-1562 gedr.; es muss alier heissen $\left.1840-1862^{4}.\right]$

- Katalog über die seit 1840 lis Iuli 1863 ausgegevenen Briefmarken aller Liinder mit beigedruckten Verkaufspreisen. Hrsg von 7. \& K. Zweite tut] Fibda. [1863] 80 S. 8". Die "Lrste AuH.“ war in der Zeitecbrift "Magazin

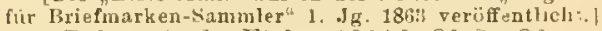
- Dritte Autl. Ebda. 1364.] $80 \mathrm{~S} .8 \%$

- - Nachtr. 1. Fbda. 1864. S.81-84. 8".

- Vierte Aufl. Ebda. [1865.] 70 S. 8*.

- - Nachtr. Ebda. [1866.] 4 S. $8^{\circ}$.

- Fïnfte Aufl. Fibda. 1866. Tfi S. $8^{\circ}$.

- Sechste Auf. Ebda. 1867. $72 \mathrm{~S} .8^{\circ}$.

- Siebente Aufl. Ebda. 1868. 71 S 8".

- Achte Auf. Ebda. 1870.79. 80

- - Nachtr. Ebda. [1871.] 8 S. $8^{\circ}$.

- Neunte Aufl. Ebda. 1872. 84 S. 8\%. Zehnte Aufl. Ebda. 1876. 60 S. 8*. - Nachtr. Hibda. [1876.] S.61-67. 80. Filfte Auf. Fibda. 1879. 80 S. $8^{\circ}$.

Zukunft, Die, der Philatelie. Vortrag geb. ven Dr. Hans Brendicke. s. Deutscher Philatelistentag; in: Stenogruph. Bericht ï. d Verhandlungen des 6. Deutschen Philatelistentages in Kiel 1894.
Yumstein, livast: Europa-liatalog 1911. Lem: Sellostverl. 1911. ... 8. 80.

— - Zweite Aufl. 1912. Wbda. 1912. ... \$. 8".

- Spezial-liatalog .und Handbuch ïber dio Briefmarlien der Schwei\%. liern: Selbstrerl. 1909. IV, 207 S. m. Abb. + 55 S. Tabellun über die Abstempelungen der Ausg. 1843 bis 1881. $8 \%$.

- Spezial-liatalog und Handbuch niber die Briefmarken der Schweizerischen Kidgenossenscluaft. Ebda. 1914. 368 S. m. Abb. $8^{0}$.

Zuschriften, den Ḱatalog betreffende, des Internationalen Postwertzeichen-HändlerVereins zu Berlin. s. Köllner, Ad, i. Nachtr.

Zustellumgsmarken der Stadtpost Warschau. (Preisnotierungen.) s. Senf, Gebrïder: Die Marken der deutschen Kolonien u. Anslandspostämter (Anhang.)

Zweck und kinriehtumg von Briefmarkenbörsen. Vortrag geh. von Dr. Hans Brendicke. s. Deutscher Philatelistentag; in: Stenograph. Bericht über den 5. D. Ph.-T. jn Berlin 1893.

Zweek und Ziele sowie Entwickelungsgang der Wissenschaftlichen Gruppe 8. Panli, Emil. (Vortrag geb. auf dem XXI Deutschen Philatelistentag zu Karlsbarl 1909.)

\section{Berichtigungen.}

Spulfes Auf der neunten Zeile von uatei usch oben muß der irrtïmlich uuter Auktions-Katgloge geatellte Titel: Kobl, Paul: IIl. Freimarken-Katalog ,Eugl. Kolonien", Abt. 3. Kolonion in Australien unter dem nichst fettgedrucklen Stichwort "Australien" z.ll steheu kommen.

Spalte of muß cy unter ,Baden" bei Rorumel, 0. : Baden. Leipzlg 1893 dahinter in Klamnern heiben: (Kritzsch, llandb. Bd. I. Abschnitt 2.) statt Bd. 2.

Spalte $\boldsymbol{i}$ desgl, unter "Bayern" Rummel, O.: Bayern (Krötzsch, Hsndb. Bd. I Abschnitt 3) statt Bd. 3.

Spulle i hoi "Bayern" lies unter: Stenger: Ersais und Proben der Brief-, Porto-, Telegraphen-, Stempelmarken u. Ganzeachen von Bayerd. Berlin "1913“ vtstt 1893 .

Spalte 10 muter ,Berlin" (Intera. Postwertzeichen-Ausstellung) Katalog der Int. Postwertz.-Ausstellung Berlio v. 25. August his 4. September $1904 \mathrm{mmB}$ dio Seitenzabl statt 101 S. , $96+30$ S." lauten, auBer dem alo Verleger der Namo "Arthur Scholem" davor gegetzt werden.

Spalte 12 rechto unten lies unter „Bechrasna, Eugen von: Dio Pogtmarken des russischen Kaiserreiches. Leipzig: Hugo Krötzsch $1895^{\prime \prime}$ statt T. 3 ,Teil ?. Auslend Bd. 1."

Spalte 13 unter "Buanschweig" muB es hei Rommel \& Krötzsch: Braungchweig, ,Bd.I. A lschnitt5" heiBen.

Spalte It desgl. unter "Bremen" bei denselben Autoren "Bd. I. Abschnitt 6."

Spalle li lies hel, ,Bund Deutscher u. Osterr. Phil. atelisteuvereine gegriudet hf dem 8. Deutguben Pbilatelisten-Tage zu Göln a. Kh. statt Juni in ,Juli" 1896.
Spalle 31 unter: Eebelmanu, P. \& Ca.: Hllugtr. Pootwertzeiclien-Katalog. Dresden: P. Wekelmann \& Co. 1892 ist dis Seitenzabl ,514 S." hinzuzufügen.

Spalle 41 hei „Friedl, Sigmund: Zur Geschichte deo Osterr. Mercure 1850-1856" jst das Erscheisuugojahr , $1895^{\prime \prime}$ hinzuzufügen.

Spalle 6.2 rechts mten lies bei Mogchkau, A.: Handb. für Postmarken-Ssmmler ststt Nachtr. 5 Leipzig 1886: ,Nachtr. 4-7. Leipzig 1886-1890."

Spalie il lies auf der vierten \%eile von links ohen nach unten ststt $76 \mathrm{~S}$. nur ,72 $\leqslant . "(\mathrm{Zu}, 2$. Auf. von Krötzвch, Ststigtik üler Stiickzalıl u. Wert moiner Postwertz.-Sammlnng.)

Spulle it Zu, Lietzow, Paul: Das schwarze Buch der I'hilatelie-6 soll eine 2. Aufl. 1886 ergchicnen seiv, worüber hisher jedwedo hibliograhigchen Angabon leblen.

Spalte 86 lies unter "Mloechkau, Alfred: Handuch ..." 3. verm u verb. Aufl, statt $268 \mathrm{~S}$. $+89 \therefore$ m. Abb.: "268 S. einschl. 89 Taf. n. Nachtr."

Spalle 91 links oben ma das Erscheiuuggjahr bei Nenlinger, Ed. จ.: Schweiz ,1888.6 statt 1890 heißed.

Spalte 9f sind bei ,Ortleb' die Aufangsbuchatalien "A. u. G." ls Vornamen hinzuzufïgen.

Spallo 98 ist bei Pirl, Paul: Leitgät\%e zur Bewertung der Austempolungen hinzuzufïged: „Schutzblatt Nr. 1 . 1912."

Spalle $10 t$ unter Redwitz, Ferdioand: GelegenleiloIfferte ist die Jafregzahl auf .,1890-1914'‘zu ergänzen.

Spulfn 115 liaks vierte Zeile von oben ngch unten zu: „Schweigor-Lercheufeld, A. Frhr. voo: Das vene Buch vou der' Weltpost" muß dso Erecheinungsjahr, ,1901". stalt [1892\} heiben. 


\title{
Nachtrag zum
}

\section{"Handbuch der deutschen philatelistischen Literatur"}

\author{
(s. H. W. u. = siehe Hauptwerk unter)
}

Afglanistan im Großen Handbuch der Phila. telie" Bd. 1. S. 27-35. Leipzig 1:87.

İypten. Teltz, Otto: Ägypten; im ,Großen Handbuch der Philatelie Bd. 1. S. 13-25. Leipzig 1887.

Andersel, Max: Die deutsche Post in der 'l'ürkei, in China und in Marokko unter besonderer Bericksichtigung der anderen fremden Postanstalten in diesคn Ländern. Mit 3 Karten. Berlin 1912, 18: S. $8^{\circ}$.

Angola im "GroBen Handbuch der Philatelie" Bd. 1. S. 39-42. Leipzig 1887.

Ansichtspostkarte, Die. s. Schwerin, L. von.

Intigua im ,GroBen Handbuch der Pbilatelie Bu. 1. S. 43-45. Leipzig 1887.

Antionuia im ,Großen Handbuch der Philatelie Bd. 1. S. 47-54. Leipzig 1887.

Argentinien. Lindenberg, Carl: Argentinische Republik; im ,GroBen Handbuch der Philatelie* Bd. 1. S. 56-72. Leipzig 1887.

Iuktions-Kataloge. s. Köhler, Heinrich; Maier, M. Kurt; Stock, Ernst.

Azoren. Kalckhoff, Franz: Azoren; im , GroBen Handbuch der Philatelie ${ }^{4}$ Bd. 1. S. 74-82 B. Leipzig 1887.

Baden. Kalckhoff, Franz: Baden; im ${ }_{\pi}$ GroBen Handbuch der Pbilatelie ${ }^{\star}$ Bd. 1. S. 83-87 A. Leipzig 1887.

Bahama-Inseln. 'Teltz, Otto: Bahama-Inseln; itm GroBen Handbuch der Philatelic ${ }^{-}$Bd. 1. S. 83-91. Leipzig 1887.

Bangkok. Teltz, Otto: Bangkok; im GroBen Handbuch der Philatelie- Bd. 1. S. 93-94. Leipzig 1887.

Barbados. Teltz, Otto: Barbados; im Großen Handbuch der Philatelie ${ }^{*}$ Bd. 1. S. 96-103. Leipzig 1887.

Bayer, Eugen: Die 5-Centavos-Kondormarke von Bolivien (Sonderdr. aus "Der Philatelist" 1918 Nr. 9). Dejvice-Prag: Selbstverl. des Verf. 1918. $4 \mathrm{~S} .8^{\circ}$.

Bayern. Kalckboff, Franz: Bayern; im „GroBen Handbuch der Philatelie ${ }^{*}$ B. 1. S. 105-134. Leipzig 1887.

Belgien. Kalckhoff, Franz: Belgien; im ${ }_{7}$ GroBen

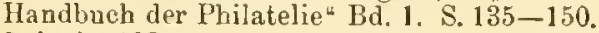
Leipzig 1887.

Bergedorf. Kalckhoff, Franz: Bergedorf; im "GroBen Handbuch der Philatelie" Bd. 1. S. 157 u. 158. Leipzig 1887.

Berlin (Berliner Philatelisten-Klub). Verträgliches Correspondenz-Blatt. Officielles Organ des V1lI. Stiftungsfestes des Berliner Pbil. atelisten.Clubs. 1.Jahrgang No.1. 19. Januar 1895. Berlin: Selbstverlag 1895. $12 \mathrm{~S}$. in farb. Umschlag. $8^{\circ}$.
Berullad. Kalckhoff, Franz: Bermuda; im "GroBen Handbuch der Philatelie" Bd. 1. S. 159-162. Leipzig 1887.

Blopal. Szamatolski, Max: Bhopal; im , GroBen Ilandbuch der Philatelie" Bd. 1. S. $163-166$. Lejpzig 1887.

Bhor. Szamatolski, Max u. Franz Kalekhoff: Bhor; im ,Grossen Handbuch der ibilatelie Bd. 1. S. 167. Leipzig 1887

Bibliotlek der Liebhabereien Bd. 1: Die Ansichtspostkarte. s. Schwerin, L. von.

- der Rechts- u. Staatskunde in gemeinver. ständl. Darstellung hrsg. von Ernst Wanke. Bd. 20: Unser Postwesen. s. Persulin, Werner.

Bickel, Carl: Mitarbeiter am ,Großen Handbuch der Philatelie“. s. 11. W. u. . Montenegro*.

Bolivar. Heitmann, Erust: Bolivar; im ,Groben Handbuch der Philatelie* Bd. 1. S. 169-173. Leipzig 1887.

Bolivia. Bayer, Eugen: Die 5-Centavos-Kondormarke von Bolivien. Dejvice-Prag 1918.

- Blaubut, Bernhard: Bolivia; im ,GroBen Handbuch der Philatelic" Bd. 1. S. 174-177. Leipzig 1887.

Booleman, M. \%.: Satz-Preisliste 1911/12. Amsterdam, 54 Rokin: M1.Z. Booleman. 16S. $4^{\circ}$. [Mit teilweise deutgehem Text]

Borek, Richard: Briefmarkenpreisliste Ausg.B. Braunschweig: Richard Borek 1918.

- Ausg. C. Narken feindlicher Staaten. Ebda. 1918.

Bosnien u. Herzegowina. Heitmann, Ernst: Bosnien; im ,GroBen Handbuch der Philatelie * Bd. 1. S. $178-181$. Leipzig 1887.

Brand, lleruann: Neueste Brielmarken-Preisliste 1883 84. Naumburg: Hermann Brand 1884.

- - Preisverzeichnis Juli 1887. Lbda. 1887.

Brasilien. Lindenberg, Carl: Brasilien; im GroBen Handbuch der Philatelie Bd. 1. S. 182-195. Leipzig 1887.

Brannsellweig. Heitmann, Erust: Braunschweig; im ,GroBen Handbuch der Philatelie" Bd.1. S. 196-201. Leipzig 1887.

Brenen. Heitmann, Ernst:Bremen; im "GroBen Handbuch der Philatelie* Bd. 1. S. 202-204. Leipzig 1887.

Brendicke, Hans: Der Briefmarken-Sammler. Hrsg. von der Redaktion des Guten Kameraden. 16.-18. 'Tausend. Stuttgart, Berlin, Leipzig: Union, Deutsche Verlagsgesellschaft 1918. 138 S. m. $203 \mathrm{Abb} .8^{\circ}$.

Hriefnarken - Versteigerungen. s. Koehler, Heinrich; Maier, M. Kurt; Stock, Ernst.

Britisch-Betseln nanenland. Lindenherg, Carl: Britisch-Betschuanenland; im GroBen Handbuch der Philatelje Bd. I. S. 205-210. Leipzig 1887. 
Brilisch-Columbia u. Vanconverinsel. Lindenberg, Carl: Britisch-Columbia v. Vancouverinsel; im ,GroBen Handbuch der Philatelie" Bi. 1. S. 211-212. Leipzig 1887.

Briliseh-fuiana. IJoffmann, Emil: Britisch. Guiana; im "Großen IIandbuch der Philatelie Bd. 1. S. 213-224. Leipzig 1887.

Briliseh-llonduras. Linden berg, Carl: BritischHonduras; im GroBen Handbuch der Philatelie ${ }^{4}$ Bd. 1. S. $225-228$. Leipzig 1887.

Britisch-Indiell. Lindenherg, Carl: BritischIndien; im "Großen Handbuch der Philatelie" Bd. 1. S. 229-250. leeipzig 1887.

Britisele-Nord-Borneo. s. Hw. Nordborneo.

Buenos Aires. Heitmann, Ernst: Buenos Aires; im GroBen Handbucl der Philatelie" Bd. 1. S 258-259. Leipzig 1888.

Bulgarien. Kropf, Hans: Bulgarien; im ,Großen Handbuch der Philatelie ${ }^{4}$ Bd. 1. S. 260-268. Leipzig $18 \pm 8$.

Bungerz, Alexander: Utber Farbenbezeichnungen in der Philatelie. Sonderahdr, aus GebrüderSenfs, Illustr. Briefmarken-Journa] t $\mathrm{Nr} .18,19 / 20,21,23,241917$ [Leipzig: Gebrüder Senf in Konm.] $36 \mathrm{~S}$. $8^{\circ}$.

Canada. Lindenberg, Carl: Canada; im, Groben Handbuch der Philatolie ${ }^{4}$ Bd. I. S. 269-280. Leipzig 1888.

Cassel (Verein für Briefmarkenkunde). Geschichte des Vereins für Briefmarkenkunde zu Cassel. Gegr. d 17. Novbr. 1881. 〈Eingetr. im Vereinsregister am 23. Juni 1917 unter Nr. 164.> Leipzig: Buchdr. August Hoffmann 1917. Im Selbstrerl. des Vereins. 24 S. $8^{\circ}$.

- s. a. Hauptkatalog der 26. Internationalen Postwertzeichen-Ausstellung Cassel vom 9.-23. August 1914.

Ceylou. Hoffmann, Emil: Ceylon; im ,Großen Handbuch der Philatelie* Bd. 1. S. 299-330. Leipzig 1888.

Chamba. Heitmann, Ernst: Chamba; im ,Großen Handbuch der Plilatelie“ Bd. 1. S. 3331-334. Leipzig 1888.

Charraste, Ignaz: Preisliste der Briefmarkenhandlung von ? Ch. in Foçsani, Rumänien. (Nur Rumänische Postwertzeichen. Foęsani: Selbstver]. 1891. $13 \mathrm{~S} .80$.

Chile. Heitmann, Ernst: Chile; im ,Großen Handbuch der Philatelie" Bd. 1. S. 335-344. Leipzig 1888.

Cicalek, Theodor: Die Kolonien des Deutschen Reichs. (Separatdr. aus dem Jahresberichte der Wiener Handels-Akademie für 1885.) Wien: Selbstrerl, der Wiener Handels-Akademie 1885. $56 \mathrm{~S} .8^{\circ}$.

Crome-Schwieuing: Über Presse und Philatelie. Sonderabdr. a. ,ill. Briefm.-Journ." Leipzig.

DeuIsche Kolonieu. Cicalek, Theodor: Die Kolonien des Deutschen Reichs. Wien 1885.

Dieua, Emilio: Mitarbeiter am "Großen Handbuch der Philatelie". s. H. W. u. .Modena". Neapel.
Diislerbehn, H.: Geschichte und Katalog der deutschen Eisenbahnmarken 1918. 3. Aut. Oldenburg: Selbstverl. 1918.

Ecksteil, E. L. Dr. Eckstein's Alt-Deutsehland-Catalog. Catalogue de l'Ancienne Allemagne. Catalogue of Old Germany. Aachen: Selbstverlag. hl. $8^{\circ}$.

$$
\begin{aligned}
& \text { Ausgabe: A Lit. A-S [1914] } 48 \mathrm{~S} \text {. } \\
& \text { B [1916]64 S. }
\end{aligned}
$$

Eisenbahumarken. s. Düsterbehn, II.

Enke, Emil: Katalog über Briefmarken, Postkarten, Couverts u. a. Postwertzeichen, welche zu den dabei bemerkten Preisen von E. F. in Leipzig, Eutritzscher Str. 12 zu beziehen sind. Leipzig: Selbstverl. d. Verf. (1887). 138 S. $8^{\circ}$.

Entwickelungsgeschichle der Posten in Deutschland. s. Hartmann, Eugen.

Farbenbezeichnungen in der Plilatelie. s. Bungerz, Alexander.

Feldpost. Kurze Geschichte der Post und der Feldposten. Siegen: Montanus-Verlag 1918. [Noch im Erscheinen,]

Fellmann, H.: Anleitung zur Abhaltung von Bundes- u. Philatelisten-Tagen. s. H. IV. u. "Philatelistentage".

Fischer, Georg: Preisliste Nr. 3 über Briefmarken, Postkarten etc. von G. F., Altona. Altona: Selbstver]. 1878, $4^{\circ}$.

Flandrin, Victor: Nitarbeiter am "Großen Handbuch der Philatelie". s. Hw. u. "NeuCaledonien".

Forlbin, A.: Briefmarken-Preisliste über französische Kolonien, Tunis u. Monaco. 3. Aufl. Paris, 80 Rue Saint Lazare. 1912. 40 S. $8^{0}$. [Mit teilweise deutschem Text.]

Französische Kolonien. Forbin, A: Briefmarken-Preisliste über französ. Kolonien,Tunis ט. Nonaco. Paris 1912.

Frascati, Rosa: Preisliste 1910. 〈vorm. Salvatore Frascati.) Triest: Selbstverl. $41 \mathrm{~S} .8^{0}$.

Friedl, Sigmund u. Carl: Project der Geld. anweisungskarte CCorrespondenzkarten-System) mit Formular der Karte. Wien: Selbst. verl.d. Verf $0 . J$. I Bl. $23 \times 29$ Centimeter $\left(8^{\circ}\right.$.)

11 ambnrig. 8 . Wesen u. Nutzen der Stadtbriefbeförderung zu Hamburg.

Hartmann, Eugen : Kntwicklungsgeschichteder Posten von den ältesten Zeiten bis zur Gegenwart mit besonderer Beziehung auf Deutschland. Leipzig: Franz Wagner 1868. 400 S. $8^{\circ}$.

Hauptkalalog der 26. Internationalen Postwertzelchen-Ausstellung Cassel in den Räumen der neuen Stadthalle vom 9.-23. August 1914. Hrsg. v. Regierungsbaumeister Genth in Cassel. Cassel: Selbstverl. der Ausstellungsleilung 1914. $92 \mathrm{~S} .8^{\circ}$.

Ilayn, Ernst: Jubiläums-Prejsliste Nr. 17 der Briefmarken Handlung von E. H. 18:2-1907. Engros - Eu détail. Naumburg (Saale) Langegasse: Ernst Hayn 1907. 32 S. $8^{\circ}$.

Heinze, E.: Altitalienische Poststempel. (Sonderabdr. a. Germania-Berichte.) 'T. 1: Modena, Parma, Toskana. Leipzig Breslau : GermaniaRing 1917. S. m. Abb. $4^{0}$.

- T. 2: Neapel. Fbda. 1917. S. m. 4 Kunstdr. Taf. $4^{0}$. 
Herbst, IIax: Káatalog. Preisnotierungen über echte Briefmarken 1917. Hamburg: Max Herbst 1917.

llyllested, Carl: Preise der Briefmarken-Hant. fung yon C II. (Bloss Skandinavische l'ostwertzeichen.) Copenhagen:1.Jan. 1881. 38 4".

- Ebda, 1889. 16 \$. 8".

- - s. a. im Hauptwerk.

Jacobi, Fritz: Die Entwickelung des P'ostwesen;. Mit 11 Illustr. u. Aufuahmen im Reichspostmuseum zu Berlin. Leitartikel in: Reclams Universum 30. Jg. 1914. H. 25.

Jalırbuclı, Polnisches, für Philatelisten, Ansiehts-Kartensammler etc. ete. 1911. Rzeszów (Galizien): Administration des Filatelista 1910. ca. 300 S. $8^{0}$.

Inhalt: Adrossen yon Sammlern der ganzen Welt, Zeitschriften, Vereinen, Börsen, Auskuftsbiiros. Verzeichnis der Fachliteratur, ausfilirl. Geldtabello aller Länder, Kalendarium, philatel. Fozyklopädie, Preis. rỉstal, Tauschangebote, Anonacen, nebst: Pràimien. Coupons.

[Mit teilweise deutschem Text.]

Ilwof, Franz: Das Postwesen in seiner Futwickelung von den ältesten Zeiten bis in die Gegenwart. Drei Vorträge von F. J. Graz: Leuschner d Lubensky 1880. 70 S. $8^{\circ}$.

Italien: Heinze, E. : Altitalienisebe Poststempel. 2 Teile. Leipzig-Breslau 1917.

Katalog der philatelistischen Zeilungsliteratur der ganzen Frde. s. Rommel, Otto.

King, Donald, A.: Mitarbeiter am „GroBen Handbueh der Philatelie". s. u. "Neu-Braunschweig", "Neu-Fundland “, ,Neu-Sehottland“ .

Köbler, Heinrich: [Katalog der] BriefmarkenAuktion Nr. 17. Stïekweise Versteigerung einer Prachtsammlung im Künstlerbaus Berlin W., Bellevuestr. 3. Versteigerungsiage: 22. bis 25. April 1918. [Nebst: Illustrationsbeilage auf Kunstdruckpapier] Berlin: Heinrich Köhler. 90 S.m. Abb. i. Text u. 24 Taf. $8^{\circ}$.

- Katalog der Briefmarken-Auktion Nr. 18. Stückweise Versteigerung einer Prachtsammlung im Künstlerhaus Berlin W., Bellevuestr. 3. Versteigerungstage: 25. bis 27. Juni 1918. [Nebst: Illustrationsbeilage auf Kunstdruckpapier]. Ebda. 1918. 95 S. m. Abb. i. Text u. 31 Taf. $8^{\circ}$.

Küllner, Ad.: Den Katalog betreffende Zuschriften des Internationalen PostwertzeichenHändler-Vereins zu Berlin. Zsgest. von Ad. K. Berlin: (Internationaler PostwertzeichenHändler Verein 1912). $15 \mathrm{~S} .8^{\circ}$.

Kïnig, Enil: Gesehichte der BriefgeleimnisVerletzungen und der schwarzen Kabinette in PreuBen-Deutschland. 1. Aufl. Bern 1878. 173 S. $8^{\circ}$.

- s. a. im Hauptwerk.

Kosack, Philipp: Amerika-Katalog 1914. Berlin: Philipp Kosaek \& Co. 1914. ... S. $8^{\circ}$.

Larisch, Inselm: Postkarten-Offerte. Wien: A. Lariseh 1887. $16 \mathrm{~S} .4^{0}$.

- Naehtr. 1. Ebda. 1887. 1 Bl. 4º.
Lileratur, philalelislische. König, Ludwig: Bericht izber philatelistische Veröffentlichungen in Tageszeitungen u. Familienjournaten. Vortrag geh. auf dem 5. Deutschen Philatelisten-Tag in Berlin 1893.

Locwi, Rulolf: Die Postanweisung. Fïrth i. Bayern: Gearg Rosenberg I892. 32 s. $8^{\circ}$.

Maier, 11. Kur1: Katalog der] BriefmarkenVersteigerung im Künstlerhaus Bellevuestr.3, Berlin W. Versteigerungstage: 24.-28. Mai 1918. [Nebst:] Illustrationsbeilage [auf Kunstdruekpapier]. Berlin: M. Kurt Maier 1918. 80 S. m. Abb. i. Text u. 15 Taf. $8^{\circ}$.

Malke, C.: lnteruationaler philatelistiseher Zeitung-Katalog s. II. W. u. Zeitungs-Katalog.

Hichel, Hugo: Europa-Katalog 1918. Weimar: Hugo Michel 1918. 202 S. m. A' b. i. Text. $8^{\circ}$. [Eıutuanà duakelrot.]

- Furopa-Katalog 1917. Nachtr. Nr. 1. April 1917. 24 S. $8^{\circ}$.

- Ni.2.

Niirbilz, ll.: lllustriertel Preiskatalog Nr. I der Btiefmarkenhandlung von H. M. DresdenA. Zugleich Nancoliste für Schwanebergeralbum (Jubel-Ausg.), Schaubek- u. ZschiescheAlbum. Dresden: Selbstrerl des IJrsg. (1889) 152 S. $8^{\circ}$.

Sormegen u. Selnweden. s. u. Skandinavien.

Ortsuamen, Dentsche, in Ungarn. Unentbelnlieher Behelf für den briefliehen Verkehr mit Ungarn. Hrsg. vom Verein zur Erbaltung des Deutschtums in Ungarn. Wien: Selbstverlag.

1'aderborn. Postwesen s. Stolte, Bernh.

Persulu, Werner: Unser Postwesen. Stuttgart: H. H. Moritz 1907. 176 S. $8^{\circ}$. (Bibliothek der Rechts- u. Staaiskude in gemeinverständl. Darstellung lurgg. von Erast Franke Bd 20.)

Poslanweisung, Die. s. Loewi, Rudolf.

Posiwesen. Die Entwickelung des Postwesens. S. Jacobi, Fritz; 11 wof Frauz; Persuhn, W erner.

presse und Philatelie. s. Crome-Sebwiening.

Redwilz, Ferdinand: Sonderpreisliste über die Wertzeichen der Deutschen l'ost in Rumänien. Stuttgart: Feerdinand Redwitz 1917.

- Weltkriegsmarken. Zusammenstellungen nur durchaus versehiedener echter Staalsmarken. Ebda. 1917.

Rödelheim (Verein für Briefmarkenkunde Gegr. 1902). Katalog der Postwertzeichen-Ausstellung September 1907. Ausstellungslokal: Turnhalle der Rödelheimer Turngemeinde, Gartenstr. 5. Rüdelheim: [A usstellungs Komitee] $1907.15 \mathrm{~S} .8^{\circ}$.

Roeder, li. I1. Das neue Postrecht, enthaltend Postordnung für das Deutsche Reich vom 28. Juli 1917 mit erläuternden Anmerkungen sowie Gesetz über das Postwesen des Deutschen Reiehs vom 28. Oktober 1871 nebst der Postnovalle vom 20. Dezember 1899. Mit einem Tabellenanhang über das P'osł gebührenwesen. Berlin: Industrieverlag Spaeth \& Linde 1917. $213 \mathrm{~S} .8^{\circ}$. 
Rollenmarken, Die, der Vereinigten Staaten von Amerika. s. Ceberall, Hans.

Romuel, 0lto: Katalog der Philatelistischen Zeitungsliteratur del ganzen Erde, Systematische nach Sprach- und Ländergruppen ge. ordnete chronologische Aufstellung der gesamten philatelistischen Zeitungsliteratur. Unter Mitwirkung des Herrn Senatspräsidenten Victor Suppantschitsch in Graz, des Herrn E. D. Bacon in South-Croydon, des Herrn Rev. R. R. Thiele in Wauwatosa, Wisc., des IIerrn A. Jacques Parès in Toulon, des Herrn E. B. Jones in Sioux. City, Jowa, und anderer, verfaBt von Dr. Rommel, Böhlitz-Ehrenber bei Leipzig. Budapest: Béla Székula. S. $8^{\circ}$.

[Uavollendet. Der deutscheprachliche Teil umfaBt die Seiten 1-34].

Rossig: Handbuch für den Postverkehr im Gebiete Ob.-Ost. Mitau: Steffenhagen \& Sohn 1918.

Rumainien. Charras, lguaz: Preisliste. (Nur Rumänische Postwertz.) Foçsani 1891.

Sehweden u. Norwegen. s. u. Skandinavien.

Schweriu, L. von: Die Ansichtspostkarte. Prakt. Anleitung f. Sammler zur Abschätzung u. Unterscheidung der Karten, sowie zum Sammeln u. Ordnen derselben, miteiner kurzen historischen Einleitung und mit mehreren Postkartenbeilagen. 2. Aufl. Leipzig: Franz Lipp 1902. V111, 83 S. m. 18 Abb. $8^{\circ}$.

(Bibliothek der Liebhabereiea Bd. 1.)

Skandinavien. Hyllested, Carl: Preisliste über Skandinav. Postwertzeichen. Copenhagen 1881 u. 1889.

- Sundberg, J. C.: Preisliste über Skandinavische Postwertzeichen. Copenhagen 1887.

- Engros-Preisliste Nr. 2 u. Skandinav. Postwertzeichen. Ebda. 1888.

- s. a. Skandinavien im Hauptwerk.

Steugor, Erielı: Die deutschen antlichen Ganzsachen der durch Deutschland besetzten Gebiete.Charlottenburg: Selbstverlag 1917.4S.4\%

- - : Die Auflagezahlen der in den besetzten Gebieten verausgabten deutschen Briefmarken. (Sonderabdr.aus der "Berl.Briefm.-Ztg." 1918 H. 11/12.) Mit Nachtrag nach dem Stande vom 15. Juli 1918. (Sonderabdr. aus H. 13/14.) Berlin: Ph. Kosack \& Co. 1918. 7 u. 2 S. $4^{\circ}$.

Stock, Frnst: [Kalalog der] Briefmarken-Versteigerung im líüstlerhaus Bellevuestr. 3, am Potsdamer Platz, Berlin W. Versteigerungstage: 25.-27. März 1918 [nebst]: Abbildungen zum Versteigerung Katalog. Berlin W. 8, Friedrichstr. 79 a: Ernst Stock 1918. 91 S. m. Abb. i. Text +25 Taf. als Extrabeil. $8^{\circ}$.
Stock, Ernst:[Katalog der] zweitenBriefmarkenVersteigerung im Künstlerhaus Bellevuestr. 3, BerlinW.Ver'steigerungstage:29.4-1.5.1918. [nebst]: Abbildungen zum VersteigerungsKatalog. Ebda. 1918. 90 S. m. Abb. i. Text +20 Taf. als Extrabeil. $8^{\circ}$.

- [Katalog der] dritten Briefmarken-Versteigerung im Künstlerbaus, Bellevuestr. 3, Berlin W.8. Versteigerungstage 29. Mai - 1. Juni 1918. [nebst]: Abbildungen zum Versteige. rungs-Katalog. Ebda. 1918. 116 S. m. Abb. i. Text +30 Taf. als Extrabeil. $8^{\circ}$.

- [Katalog der] 4. Briefmarken-Versteigerung im Künstlerhaus Bellevuestr. 3, Berlin W. 8. Versteigerungstage 30 . Septbr.-2.0ktbr. 1918 [uebst]: Abbildungen zum VersteigerungsKatalog. Ebda. 1918. 91 S. m. Abb. i. Text + 16 Taf. als Extrabeil. 80.

Stolte, Bernlıralt: Beiträge zur Geschichte des Postwesens im ehemaligen Hochstifte Paderborn. Paderborn: Ferdinand Schöningh 1891. 61 S. $8^{\circ}$.

Sundberg, J.C.: Preisliste über Skandinavische Briefmarken etc. von J. C. S. Copenhagen: Selbstver1. 1887. 16 S. $8^{\circ}$.

Engros-Preisliste Nr. 2 über Skandinavische Briefmarken etc. Ebda. 1888. $16 \mathrm{~S} .8^{\circ}$.

Szaualolski, Max: Mitarbeiter am "Großen Handbuch der Philatelie" s. u. "Bhopal $u$. Bhor".

Ueberall, llaus: Die amtlich verausgabten Rollenmarken der Vereinigten Staaten von Amerika. (Sonderdr. aus der "Deutschen Briefmarken-Zeitung ${ }^{4}$ H.4/5, Jg. 1918). Chemnitz: Selbstverlag 1918. 11 S. m. 3 Ahh. $8^{\circ}$.

Vereinigte Slaaten ron Amerika. Kosack, Philipp: Amerika-Katalog 1914. Berlin 1914.

-- Ueberall, Hans: Die amtlich verausgabten Rollenmarken der Vereinigten Staaten von Amerika. Chemnitz 1918.

Versleigerungs-Kataloge. s. Köhler, Heinr.; Maier, M. Kurt; Stock, Ernst.

Was der Briefmarkensammler braucht und was er wissen muB. Leipzig: Theodor Hagen 1912. 48 S. $8^{\circ}$.

Weseu und Nutzen der Stadtbriefbeförderung zu Hamburg yon der Direction der Stadtbriefbeförderung. Hamburg: Paul Siegert o. J. 16 S. $8^{0}$.

Jeitungsliteralur, philatelistische. Katalog darüber s. Rommel, Otto.

\section{Nachträgliche Berichtigungen zum Hauptwerk}

Spalte 75 unter: Liadenberg, Carl: Aur vergangeaea Tagea lieg "Berlin: Berliger Ganzachen SammlerVereia" st thelbstrerl, des B. Ph,-Kl.
Spalte 104 unter: Relchspostsmt ist bei Post- aad Telegraphen-Nachrichten die Seiteazabl zu ergänzea is , , so S." 


\section{Ein papiernes Denkmal.}

Mit der zelunten Lieferung ist das Handbuch der deutschen philatelistischen Literatur beendet. Im Kriegsjahr 1915 begonnen (siehe Seite 132), trotz aller Schwierigkeiten, die uus der Weltkrieg hindernd in den Weg legte, im Februar 1918 beendet. Es ist uns eine Ehrenpflicht, allen Mitarbeitern an dieser Stelle unsern wärmsten Dank auszuspreehın; zunächst natiörlich den Herren, deren Namen das Titelblatt ziert. Wenn auch nicht alle glejchmäßig eiferfrendig an der Arbeit beteiligt waren, so lag dies in der Natur der Sache: diesen legte der Dienst an der Front, jenen der vielleicht nicht minder beschwerliche Heimatdienst Fesseln an, alle waren jedoch aufs ejfrigste hemüht, unser Werk zu erfrenlichem Ende zu führen. Dank, lierzlichster Dank allen! Nicht minderer Dank gebïhrt der umsichtigen Leitung der Druckerei Hesse \& Becker in Leipzig. Welche Arbeitsleistung diese in den scluweren Kriegsjahren vollendet hat, kann nur der beurteilen, welcher weiß, wie groß die Schwierigkeiten wareu, die überwunden werden mußten. Zuletzt, doch nicht als Letzter sei bedankt der rührigc und fleißige Bearbeiter des Werkes, welcher auch die erste Anregung gegeben hat: Max Ton. Mit Bienenfleiß hat er alles zusammengetragen, unermüdlich, trotz des heimatlichen $\mathrm{Heeresdienstes.} \mathrm{Und} \mathrm{nun} \mathrm{möge} \mathrm{es} \mathrm{hinaus-}$ gehen, unser Handbuch, in die philatelistische Sammlerwelt, als geringer Zeuge von deutscher Friedensarbeit iu Kriegszeit. Möge es dem unterzeichneten Verein neue Freunde zuführen.

\section{Der Internationale Philatelisten $=$ Verein Dresden}

I. A.: Franz Wallner, Schriftleiter. 


\section{Internationaler Philatelisten-Verein Dresden.}

Sitz: Dresden. Gründungsjahr: 1877.

Größter und angesehenster Philatelisten=Verein

mit seinen Ortsgruppen verbreitet über die ganze bewohnte Erde.

Fachzeitschrift: DER PHILATELIST. Schriftleitung: FRANZ WALLNER, Blasewitz=Dresden, Schulstraße 3. - Zusendung jeden Monat frei an sämtliche Mitglieder.

VEREINSZWECKE :

Hebung und Förderung der Briefmarkenkunde :: Unter. stützung durch Benachrichtigungen usw. :: Größte Vereins bücherei :: Kampf gegen dic Fälscher :: Freiaufnahme von Postwertzeichengesuchen : Ehrenurkunden :: Vertrauliche Mitteilungen :: Schwarzes Buch

\section{ANDERE ZWECKE :}

Geselliger ZusammenschluB :: Fachwissenschaftliche Vorträge. Vorteile beim Erwerb ganzer Sammlungen und einzelner Marken, sowie Besorgung der Neuerscheinungen durch die Kaufvereinigung :: Prüfungsstellen :: Dublettenaustausch mit Raritätenabtejlung : Vereinsalbum :: gr. Fälschungensammlung

Aufnahmebedingungen: Vollendetes 21. Lebensjahr, Jahresbeitrag: 6 Mark und 3 Mark

Kriegszuschlag für den "Philatelist", wofür die Zeitung und die obengenannten Vorteile.

Nähere Auskunft u. Probenummern durch die Firma Hans Naumann, Dresden=A., Viktoriastr. 10.

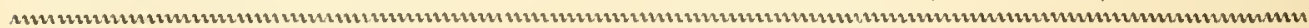

\section{Aufforderung zum Beitritt.}

Wer irgendeinen Sammelsport treibt, ist auf den Verkehr mit Fachgenossen augewiesen. So auch der Philatelist. Wir möchten uns daher gestatten, Ihre Aufmerksamkeit auf deu

\section{Internationalen Philatelisten $=$ Verein Dresden}

zu lenken, der Freunde der Briefmarkenkunde in allen Teilen der Welt zu seinen Mitgliedern zählt und der die stattliche Reihe von 29 Ortsgruppen*) anfzuweisen vermag. Der Vereiu kann auf ein mehr als 40 jähriges Bestehen zurickhlicken. Die groBe Beliehtheit, deren sich der, Internationale PhilatelistenVerein Dresden"erfreut, diirfte in den zahlreichen Vorteilen begrüudet sein, die er seinen Mitgliedern bietet.

Der ,Internationale Philatelisten-Verein“ bietet seinen Nitgliedern gegen einen Jahresbeitrag von nur 11. 9. - (ohne Erheloung eiver Aufnabmegcbiihr) folgendes:

Der, ,Internationale Philatelisten-Verein“6 lı̈ilt regelmäßige Vereinssitzungen ab.

Der ,Internationale Philatelisten-Verein" übermittelt seinen Mlitgliedern in Friedenszeiten alljährlich eine "Vertrauliche Mitteilung“, in welcher die Gesehichte des Vereins, die Regeln fïr den II andel mit Postwertzeicheu, der Katalog der Vereinsbieherei, der Katalog des Vereinsarchivs, die Bedingungen der Prüf ungsstellen für Postwertzeichen, die Bezugbedingungen für das

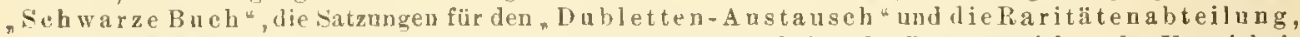
die Satzungen drr K il u fvereinjgug zur Besurgug neu erscheinender Postwertzeichen, das Verzeichnis der Mitglieder, welche die neu erscheinenden Postwertzpichen der Länder besorgen, in denen sie wohnen, und das Mitglieder-Verzeichnis enthalten sind.

Der ,Internationale Philatelisten-Verein " sorgt durch Herausgabe der heliebten, weit verbreiteten illustrierten Vereins-Fachzeitschrift .Der Philatelist" dafï, da B die Mitglieder üher alle neu erscheinenden Postwertzeichen rasch belehrt und durch Aufsätze aus der Feder erster Fachschriftsteller gründlich untrrichtet werden. Ausführlivhe Berichte drs Stanuvereins und seiner Ortsgruppen geben den vereinzelt wohnenden Mitgliedern immer Kunde vou allen wielitigen, philatelistischen Varkommnissen, Besclulüssen nsw. Im Vereins-Organ werden ferner alle neuentdeckten Fälschungen besprochen und somit die Leser vor Schaden bewahrt.

Der „Internationale Philatelisten-Verein“ liefert seinen neu eintretenden Mitgliedern anf Verlangen — soweit der Varrat reicbt _ das Handbuch der deutschen philat. Literatur, welches er nnter Mitwirkung der hervorragendsten Philatelisten herausgegeben liat, zum Preise van 5 NIark.

Der ,Internationale Philatelisten-Verein" ist im Besitz der größten Fachbücherei, aus der einzelne Bände entliehen werden können, sowie einer graßen Vereinssammlung echter Marken und einer aweiten mit nur Fälschungen; auch bietet er seinen Mitgliedern durch einen vorzüglich geleiteten, ,DublettenAustausch mit Raritätenabteilung" eine iiberaus giunstige Gelegenheit zur Verwertung ihrer Dubletten, sowie zur preiswerteı Erwerbung felılender Stijcke und gibt durch seine „Kaufvereinigung*" die IIöglichkeit, sich die neuerscheinenden Postwertzeichen aller Länder ungebrancht zum Nennwerte zu verschaffen.

Der ,Internationale Philatelisten-Verein" setzt seine Mitglieder dureh Ansgabe eines sogenannten "Schwarzen Buches" in den Stand, sich iiber unreelle Persönlichkeiten zu belehren.

Der, Internationale Philatelisten-Verein“" zählt die hervorragendsten Sammler und Händler zu seinen Mitgliedern.

Wir hoffen, dab. Sie sich entschlieBen werden, dem „Internationalen Philatelisten-Verein" als Mitglied beizutreten, und bitten Sie in diesen Falle ihre Anmeldung bei Hans Naumann, Dresden-A. I, Viktoriastraße 10 , bewirken zu wallen.

\section{Der Vorstand des Internationalen Philatelisten-Verein Dresden Baurat Falck, Varsitzender.}

* Iufolge des Krieges müssen wir vorläug die Ortagruppen foindlicher Lünder ausschalten. 



